\title{
Orientation-dependent energy transfer in gas-surface collisions: Scattering of vibrationally excited nitric oxide from $\mathrm{Au}(111)$
}

\author{
Dissertation \\ zur Erlangung des mathematisch-naturwissenschaftlichen Doktorgrades \\ "Doctor rerum naturalium" \\ der Georg-August-Universität Göttingen \\ im Promotionsprogramm Chemie \\ der Georg-August University School of Science (GAUSS)
}

vorgelegt von

Nils Bartels

aus Achim

Göttingen, 2015 
Betreuungsausschuss:

Prof. Dr. Alec M. Wodtke, Dynamics at surfaces, Institut für Physikalische Chemie Göttingen/ Max-Planck-Institut für Biophysikalische Chemie

Prof. Dr. Dirk Schwarzer, Reaction Dynamics, Max-Planck-Institut für Biophysikalische Chemie

Mitglieder der Prüfungskomission:

Referent: $\quad$ Prof. Dr. Alec M. Wodtke, Dynamics at surfaces, Institut für Physikalische Chemie Göttingen / Max-Planck-Institut für Biophysikalische Chemie

Korreferent: Prof. Dr. Dirk Schwarzer, Reaction Dynamics, Max-Planck-Institut für Biophysikalische Chemie

Weitere Mitglieder der Prüfungskommission:

Prof. Dr. Martin Suhm, Suhm group, Institut für Physikalische Chemie Göttingen

Prof. Dr. Thomas Zeuch, Aggregation and Kinetics of Reactive Systems, Institut für Physikalische Chemie Göttingen

Prof. Dr. Jörg Schroeder, Schroeder group, Institut für Physikalische Chemie Göttingen

Prof. Dr. Jürgen Troe, Niedersachsen Research Professor, Institut für Physikalische Chemie Göttingen

Tag der mündlichen Prüfung: 07.07.2015 


\begin{abstract}
The work in my thesis is a contribution to the field of chemical dynamics at surfaces. In this field we seek to develop a detailed microscopic understanding of chemical events taking place on surfaces. Progress in this field has been spurred by a fruitful interplay between experimental work and theory. My work continues in this tradition. The starting point was a striking theoretical prediction and the measurements I made test that prediction and also provide many new discoveries which I hope will help stimulate improvements in theory.
\end{abstract}

I focused on a hot topic in this field - the breakdown of the Born Oppenheimer approximation and the role of non-adiabatic electronic energy transfer in surface dynamics. Specifically, I studied the scattering of vibrationally excited nitric oxide (NO) from $\mathrm{Au}(111)$, an important and extensively studied model system for non-adiabatic dynamics. Upon collision with the surface, vibrationally excited NO molecules very efficiently transfer vibrational energy to electronic degrees of freedom in the metal, a striking case of electronic non-adiabaticity (Born-Oppenheimer breakdown), which is believed to be driven by a transient electron transfer (ET).

I measured ro-vibrational state distributions of NO molecules prepared in excited vibrational states $\left(v_{\mathrm{i}}=3,11\right.$, and 16) after scattering from $\mathrm{Au}(111)$ as function the incidence translational energy $\left(E_{\text {trans }}^{\mathrm{i}}=0.05-1 \mathrm{eV}\right)$ and orientation. The goal was to investigate the influence of these parameters on the ET-driven energy transfer and, on a more general level, to improve our understanding of the rules that govern the dynamics of molecules at metal surfaces. To make these measurements, I contributed to the development of two experimental techniques: 1) a new method to orient polar molecules in the laboratory frame (optical state selection with adiabatic orientation) and 2) a new method to improve the quantum state purity in optical pumping (pump-dump-sweep).

The effect of orientation is both dramatic and complicated. For NO with $v_{\mathrm{i}}=3$ and 11 , vibrational relaxation is significantly enhanced for molecules pointing with the $\mathrm{N}-$ atom towards the surface compared to molecules oriented with the $\mathrm{O}$-atom towards the surface. For these states vibrational relaxation is furthermore promoted by incidence 
translational energy. Interestingly, for NO $v_{\mathrm{i}}=16$ neither the orientation or incidence energy have an effect and all molecules relax to lower vibrational states. NO $v_{\mathrm{i}}=16$ has no survival probability in its initial vibrational state. Rotational state distributions of surface scattered molecules exhibit pronounced rotational rainbow structure that strongly depends on the incidence translational energy, initial orientation, and final vibrational state. These are the first observation of rotational rainbows for molecules that have undergone vibrational relaxation.

The measurements have a complicated dependence on orientation, initial energy and vibrational state. Nonetheless, the trends in the vibrational relaxation probability can be understood in terms of a simple model based on the barrier in the energetics of the underlying electron-transfer reaction. Vibrational relaxation requires overcoming this barrier. The barrier decreases as the initial vibrational state increases. This explains the trend to stronger vibrational relaxation as $v_{\mathrm{i}}$ is increased. The variation in barrier height also explains the fact that translational energy promotes vibrational relaxation for $v_{\mathrm{i}}=3$ and 11 , but is not required for $v_{\mathrm{i}}=16$. The barrier is lowered by a favorable $\mathrm{N}$-atom first orientation, resulting in the dramatic increase in relaxation for $\mathrm{N}$-atom first collisions.

The experimental data from this work provides a valuable test for theory. Recent calculations of vibrational relaxation based on electronic friction or independent electron surface hopping fail to explain the final vibrational state distributions and how they vary with incidence energy and orientation. My hope is that these measurements will stimulate further theoretical work and new insight into the dynamics of this important example of non-adiabatic chemical dynamics at surfaces.

An independent topic covered in my thesis is the generation of a molecular beam of highly vibrationally excited $\mathrm{CO}$ using perturbations. This technique is called pumppump-perturb and dump and I demonstrate its successful implementation in our laboratory. 


\section{Kurzfassung}

Meine Doktorarbeit ist ein Beitrag zum Gebiet der Reaktionsdynamik an Oberflächen. In diesem Forschungsbereich wird daran gearbeitet, ein detailliertes mikroskopisches Verständnis von chemischen Prozessen an Oberflächen zu erzielen. Fortschritt resultierte dabei häufig aus der Zusammenarbeit von experimentell und theoretisch arbeitenden Forschern und die vorliegende Arbeit führt diese Tradition weiter. Ausgangspunkt meiner Untersuchungen war eine bemerkenswerte theoretische Vorhersage, die ich experimentell überprüft habe. Dabei habe ich mehrere neue Beobachtungen gemacht, von denen ich hoffe, dass sie zu Weiterentwicklungen in der theoretischen Chemie beitragen.

Ein aktuelles und wichtiges Thema auf das ich meine Forschung gerichtet habe, ist das Versagen der Born-Oppenheimer Näherung und die damit einhergehenden elektronisch nichtadiabatischen Energietransferprozesse an Oberflächen. Dafür habe ich das Streuverhalten von hoch schwingungsangeregtem Stickstoffmonoxid (NO) an der (111)-Oberfläche eines Goldkristalls untersucht. Dieses System war bereits vor dieser Arbeit ein bedeutendes Modellsystem für nichtadiabatische Prozesse an Oberflächen. Beim Stoß mit der Oberfläche geben die hochschwingungsangeregten NO Moleküle Schwingungsenergie an elektronische Freiheitsgrade im Metall ab. Dieser sehr effiziente Energietransfer kann nur im Rahmen eines nichtadiabatischen Effektes (Versagen der Born-Oppenheimer Näherung) erklärt werden und der aktuelle Stand der Forschung ist, dass dieser Prozess durch einen transienten Elektronentransfer (ET) von der Oberfläche zum Molekül verursacht wird.

Gemessen habe ich Schwingungs- und Rotationsverteilungen von in verschiedenen angeregten Schwing-ungszuständen $\left(v_{\mathrm{i}}=3,11\right.$, und 16) präparierten NO Molekülen nach dem Stoß mit der Au(111) Oberfläche. Dabei wurden die einfallende Translationsenergie $\left(E_{\text {trans }}^{\mathrm{i}}\right)$ und die Orientierung der Moleküle systematisch variiert. Dies sollte den Einfluss dieser Parameter auf den durch ET getriebenen Energietransfer aufzeigen, sowie generell zu einem besseren Verständnis der Gesetzmäßigkeiten beitragen, nach denen Prozesse an Oberflächen ablaufen. Um die angestrebten Experimente durchführen zu können, habe ich an der Entwicklung von zwei neuen experimentellen Methoden mitgewirkt: 1) einer Methode um polare Moleküle in einem elektrischen Feld auszurichten 
(„Optische Zustandsselektion mit adiabatischer Orientierung“) und 2) einer Methode für die Unterdrückung von spontaner Emission beim optischen Pumpen („PumpDump-Sweep“).

Der Einfluss der Orientierung ist sowohl drastisch als auch komplex. Für NO in $v_{\mathrm{i}}=3$ und 11 wird die Schwingungsrelaxation stark durch eine Ausrichtung der Moleküle mit dem N-Atom in Richtung der Oberfläche gegenüber einer Ausrichtung mit dem OAtom in Richtung der Oberfläche verstärkt. Für diese Zustände nimmt die Relaxation außerdem mit steigender einfallender Translationsenergie zu. Interessanterweise verschwinden sowohl der Einfluss der Translationsenergie, als auch der Orientierung für NO in $v_{\mathrm{i}}=16$ und alle Moleküle relaxieren zu niedrigeren Schwingungszuständen. Die Rotationsverteilungen der gestreuten Moleküle hängen ebenfalls stark von der einfallenden Translationsenergie, Orientierung sowie dem Schwingungszustand ab und zeichnen sich durch ausgeprägte nicht-thermische Maxima („Rotationsregenbögen“) aus. Dies ist die erste Beobachtung von Rotationsregenbögen in Molekülen, die zuvor Schwingungsenergie durch einen Stoß mit einer Oberfläche verloren haben.

Die Messergebnisse weisen eine komplizierte Abhängigkeit von Orientierung, Einfallsenergie und Schwingungszustand auf. Trotzdem können die beobachteten Trends in der Schwingungsrelaxationswahrscheinlichkeit mit einem einfachen Modell verstanden werden, welches auf einer energetischen Barriere der zugrundeliegenden Elektronentransferreaktion basiert. Die Höhe dieser Barriere nimmt mit zunehmender einfallender Schwingungsenergie ab. Dies erklärt den Trend hin zu stärkerer Schwingungsrelaxation, wenn $v_{\mathrm{i}}$ erhöht wird. Die Änderung der Barriere erklärt auch, warum die Schwingungsrelaxation für $v_{\mathrm{i}}=3$ und 11 stark von der Translationsenergie beeinflusst, für $v_{\mathrm{i}}=16$ hingegen kaum benötigt wird. Die Barrierenhöhe wird außerdem durch eine Ausrichtung der Moleküle mit dem N-Atom in Richtung der Oberfläche verringert und führt zu einem starken Anstieg der Relaxation für diese Orientierung.

Die experimentellen Daten aus dieser Arbeit ermöglichen einen sehr detaillierten Test für Methoden der theoretischen Chemie. Aktuelle Berechnungen zu dem untersuchten System basierend auf den Methoden der Elektronischen Reibung („electronic friction“) oder IESH (,independent electron surface hopping “) scheitern bei der Vorhersage der 
erzeugten Schwingungsverteilungen und deren Abhängigkeiten von den Bedingungen des Streuexperiments. Ich hoffe, dass die neuen Daten als Grundlage für weitere theoretische Arbeiten dienen, um so noch tiefere Einblicke in dieses bedeutende Beispiel eines nichtadiabatischen Prozesses in der Oberflächendynamik zu erhalten.

Ein unabhängiges Thema, welches in der vorliegenden Arbeit zusätzlich behandelt wird, ist die Erzeugung eines Molekularstrahls hoch schwingungsangeregter CO Moleküle. Dafür habe ich eine Methode entwickelt, die „Pump-pump-perturb and dump“ genannt wird und auf dem optischen Pumpen in erster Näherung dipolverbotener elektronischer Übergänge beruht. Ich demonstriere die erfolgreiche Implementierung der Methode in dem bestehenden Versuchsufbau. 



\section{Acknowledgements}

In the first place, I would like to thank Prof. Alec M. Wodtke for giving me the opportunity to work in his group and for his way of leadership based on giving me and others a lot of freedom in research, a lot of acknowledgement for our work and a lot of trust. I would also like to thank Prof. Dirk Schwarzer for his support and for being the second reviewer of my thesis. Next, I would like to thank Dr. Tim Schäfer. We did a lot of research together. Tim shared his entire scientific, technological and personal knowledge with me and spreads happiness and enthusiasm wherever he goes.

Then I would like to thank Bastian Krüger who took over the responsibility for the experimental setup that I have been working with. He is an outstanding scientist and we measured and analysed a lot of data together.

I also want to thank all other people working in the Wodtke group and in particular the Bachelor students who contributed to results presented in this work (Sven Meyer, Jens Hühnert and Niklas Henning) and all others I have been working with in the laboratory: Dr. Li Chen, Dr. Pranav R. Shirhatti, Isabel Bejenke, Dr. Kai Golibrzuch, Nils Hocke, and Dr. Russel J. Cooper. From the theory side I would like to acknowledge Dr. Christof Bartels and Dr. Alexander Kandratsenka for their useful discussions, for comparing experimental data to computational models and Alexanders' introduction to simple molecular dynamics simulations.

My special thanks goes to Prof. Daniel J. Auerbach for his help in understanding surface science over the past years. Although he could also just enjoy life on his back-porch, he is frequently travelling to Germany and conferences around the world and helped in interpreting new data and by improving our manuscripts.

I would like to acknowledge Prof. Robert W. Field for his patience in explaining molecular perturbations and for giving me the opportunity to visit 
his laboratory at the MIT.

I would like to also thank my academic and scholar teachers, in particular Prof. Martin Suhm and Prof. Peter Botschwina (who unfortunatley deceased recently) for awakening my fascination for physical chemistry.

I also want to acknowledge the mechanical and electronic work shops at the Institute of Physical Chemistry and our mechanic meisters within the workgroup Reinhard Bürsing and Florian Lange for their technical support and fast developments. 


\section{Contents}

1 Introduction 1

2 Theory and previous results 5

2.1 Introdution to electronically non-adiabatic processes . . . . . . . 5

2.1.1 Theory and non-adiabatic effects at avoided curve crossings in the gas phase . . . . . . . . . . . . 5

2.1.2 Non-adiabatic effects at metal surfaces . . . . . . . . . . . 10

2.2 Electron transfer energetics at metal surfaces . . . . . . . . . . . 13

2.3 Vibrational dynamics at metal surfaces . . . . . . . . . . . . . . 16

2.3.1 Vibrational dynamics of adsorbed molecules . . . . . . . . 16

2.3.2 Adiabatic versus non-adiabatic vibrational dynamics in gas-surface collisions . . . . . . . . . . . . . . . . . 18

2.3.3 Vibrational dynamics of $\mathrm{NO}$ scattering from $\mathrm{Au}(111) \ldots \ldots$

2.4 The Huang experiment . . . . . . . . . . . . . . . . . . 23

2.4 .1 Energy transfer in the Huang experiment . . . . . . . . . . 23

2.4.2 Electron transfer in the Huang experiment . . . . . . . . . 26

2.4.3 Theoretical calculations for the Huang experiment and their pre-

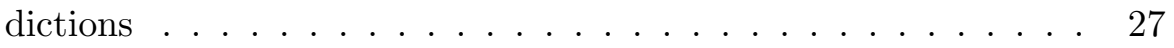

2.4.4 The challenge of testing the theoretical predictions . . . . . . 30 30

2.5 Orientation in surface dynamics . . . . . . . . . . . . . . . 31

2.6 Rotational excitation in gas-surface collisions . . . . . . . . . . 33

3 Experimental 39

3.1 Vacuum chamber with molecular beam . . . . . . . . . . . . 39

3.2 Surface preparation and verification of cleanliness . . . . . . . . . . 4 41 
3.3 Laser systems . . . . . . . . . . . . . . . . . . . . . . 43 43

3.3.1 Fourier-transform limited IR source . . . . . . . . . . . 43

3.3.2 Home-built narrow-bandwidth optical parametric oscillators . . 44

3.3 .3 Dye laser . . . . . . . . . . . . . . . . . . . . . . . . 46

3.3.4 Sunlite Ex OPO with FX-1 UV frequency extension . . . . . . 46

3.4 Data acquisition and processing . . . . . . . . . . . 47

4 Orienting polar molecules without hexapoles 49

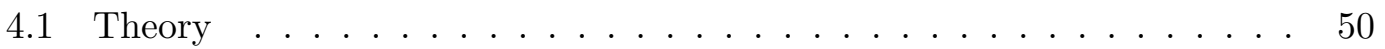

4.2 The traditional approach: Orientation via hexapole state selection . . . 54

4.3 The new approach: Optical state-selection with adiabatic orientation . . 55

4.4 Testing the method and determining the achieved degree of orientation . 58

4.5 Orientation for different vibrational states in the NO molecule . . . . . 60

5 Suppression of spontaneous emission in the optical pumping of molecules: Pump-dump-sweep

5.1 Introduction and description of the pump-dump-sweep concept . . . . 6 68

5.2 Implementation of the method and quantifying quantum state purity . . 70

5.3 Effect of the improved quantum state purity on molecule-surface scat-

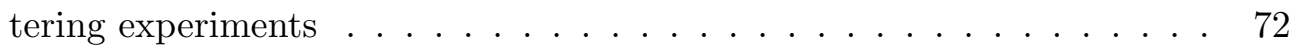

5.4 Comparison of the pump-dump-sweep approach to stimulated Raman adiabatic passage (STIRAP) . . . . . . . . . . . 74

6 Vibrationally inelastic scattering of oriented NO from $\mathrm{Au}(111)$

6.1 Vibrationally inelastic scattering of $\mathrm{NO}(v=3)$ from $\mathrm{Au}(111) \ldots . . .81$

$6.1 .1 \quad$ Experimental . . . . . . . . . . . . . . . . . . . 81

6.1.2 Probing the steric effect using method $1 \ldots \ldots$. . . . . . 84

6.1.3 REMPI spectra from oriented scattering using method 2 . . . . 86

6.1.4 Analysis of ro-vibrational state distributions . . . . . . . . . 89

6.1.5 Rotational cooling upon vibrational relaxation . . . . . . . . 92

6.1.6 Incidence energy of translation dependence of the steric effect . . 92

6.1.7 Incomplete equilibration of spin-orbit states . . . . . . . . . . 95

6.2 Vibrationally inelastic scattering of NO prepared in high vibrational states from $\mathrm{Au}(111) \ldots \ldots \ldots \ldots$. . . . . . . . . . . . . . . . . . 
6.2 .1 Experimental overview . . . . . . . . . . . . . 97

6.2.2 Example of a REMPI spectrum . . . . . . . . . . . . . . 99

6.2.3 Concept for the analysis of ro-vibrational state distributions . . 101

6.2.4 Incidence translational energy dependence of the vibrational relaxation . . . . . . . . . . . . . . . . 103

6.2.5 Orientation dependence of the vibrational relaxation . . . . 106

6.2.6 Rotational state distributions: Rotational rainbow scattering and rotational cooling upon vibrational relaxation . . . . . . . . . 108

6.3 Discussion . . . . . . . . . . . . . . . . . . 111

6.3.1 Vibrational relaxation in the framework of an electron transfer model . . . . . . . . . . . . . . . . . . 111

6.3.2 Rotational rainbows and the origin of rotational cooling . . . . 113

6.3.3 Dynamical steering at low translational energy . . . . . . . . . 117

6.3.4 The possibility of NO bond dissociation . . . . . . . . . . 118

6.3.5 A brief comparison to theoretical models . . . . . . . . . . . . . . 119

6.3.5.1 Independent electron surface hopping (IESH) model from the Tully group . . . . . . . . . . . . . . . 119

6.3.5.2 Electronic friction (EF) model from the Saalfrank group 124 6.3.5.3 Monte Carlo wave packet study from Li and Guo . . . . 124

6.4 Conclusions . . . . . . . . . . . . . . . . . . 125

7 Production of a molecular beam of highly vibrationally excited CO using perturbations

7.1 Introduction and description of the concept . . . . . . . . . . . . . 128

7.2 Experimental . . . . . . . . . . . . . . . . . . 130

7.3 Results . . . . . . . . . . . . . . . . . . . . 134

7.4 Discussion . . . . . . . . . . . . . . . . . 139

7.5 Conclusions . . . . . . . . . . . . . . . . . . . 141

8 Outlook

References 
A Basics of angular momentum coupling and an effective Hamiltonian for diatomic molecules

A.1 Angular momentum operators . . . . . . . . . . . . . . . . . 155

A.2 Coupling of two angular momentum vectors: Clebsch-Gordan Coefficients and $3 \mathrm{j}$-Symbols $\ldots \ldots \ldots \ldots \ldots \ldots$

A.3 Transformation under rotation . . . . . . . . . . . . . . 158

A.4 Wave function of a rigid rotor . . . . . . . . . . . . . 159

A.5 Hund's case a) basis functions . . . . . . . . . . . . . . . . 161

A.6 Effective Hamiltonian for diatomic molecules . . . . . . . . . . . . 162

B Calculation of mixing coefficients and lifetimes of the $\mathrm{CO} \mathrm{e}^{3} \Sigma^{-}(v=12)$ levels interacting with $\mathrm{A}^{1} \Pi(v=8)$

C Time delays in a pump-dump-sweep-probe molecular beam scattering experiment

D Mathematica notebook for calculating line positions in the $\gamma$-bands of NO

E Further details on the analysis of REMPI spectra from scattering $\operatorname{NO}\left(v_{\mathrm{i}}=11,16\right)$ from $\mathrm{Au}(111)$ 


\section{1}

\section{Introduction}

The wide application and enormous economic importance of surface chemistry have triggered the desire for detailed and predictive molecular level understanding of how systems evolve dynamically as a function of time, based on physical concepts such as quantum mechanics, energy, momentum, electron transfer or the principle of detailed balance. In order to obtain this understanding, simple systems - small molecules at clean, well defined surfaces under ultra-high vacuum (UHV) conditions - are studied and the results are compared to expectations from the above mentioned physical concepts. This research belongs to the field of surface dynamics.

A particular experiment that has created a lot of interest in this field, is the vibrational relaxation occurring when highly vibrationally excited nitric oxide (NO) is scattered from a $\mathrm{Au}(111)$ surface. Data existing for this experiment prior to my Ph.D. project is shown in Fig. 1.1. The striking observation is that scattering from the Au (metal) surface leads to an extremely efficient vibrational relaxation, whereas molecules scattering from the insulator lithium fluoride $(\mathrm{LiF})$ loose almost no vibrational energy to the surface.(1)

The surface science community explains this difference by electronically non-adiabatic transitions (Born-Oppenheimer breakdown) induced by a coupling of the nuclear motion (vibration) to electronic degrees of freedom in the metal.(1, 2) In other words, the vibrational energy is transferred to electron-hole pairs. Of course, this mechanism is not possible on the insulator. A concept, that I will support throughout this thesis, is 

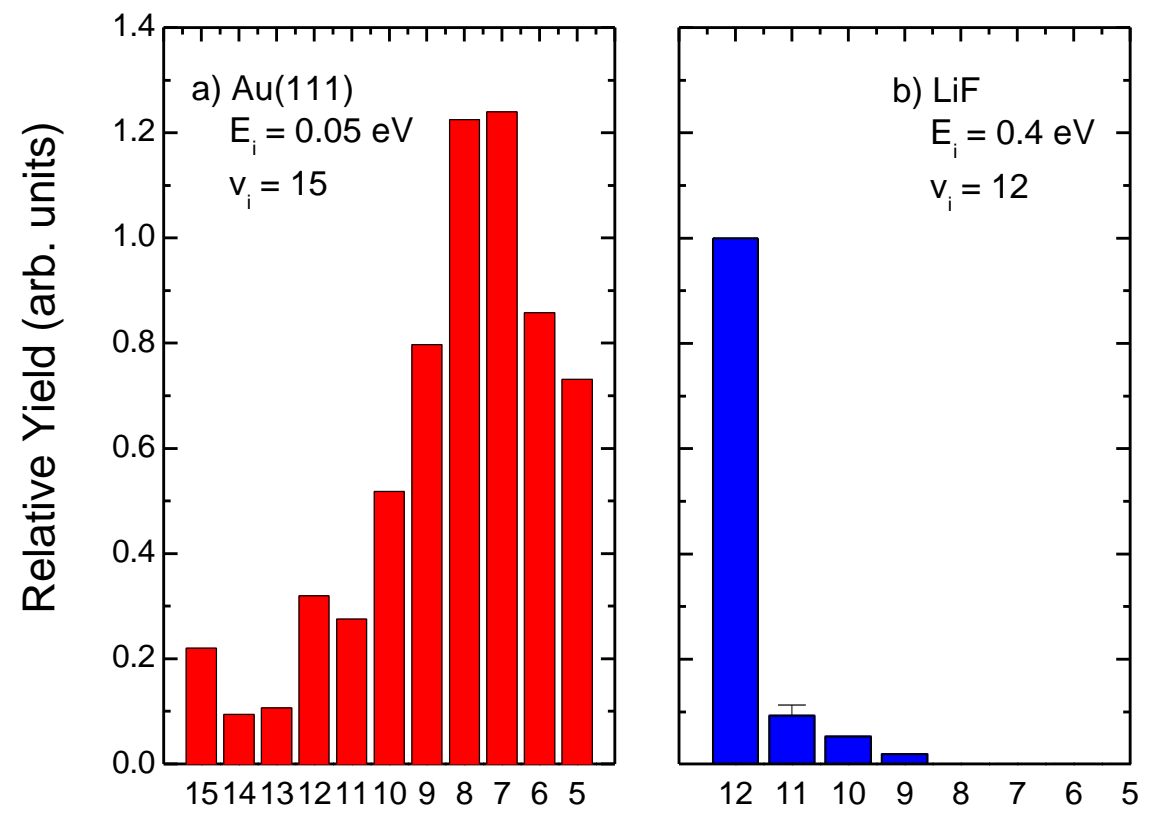

Final Vibrational State

Figure 1.1: Vibrational state distributions from the "Huang experiment" Measured vibrational state distributions of $\mathrm{NO}$ molecules scattered a) from $\mathrm{Au}(111)$ and b) from $\mathrm{LiF}$ at the incidence translational energies $E_{I}$ and the incidence vibrational states $v_{I}$ as indicated. From (1). Reprinted with permission from AAAS. 
that the non-adiabatic energy transfer is driven by an electron transfer event (transient formation of an anion).

The idea to study the effect of the vibrational relaxation as a function of the incidence orientation of the NO molecule was suggested by theoretical chemists. I would like to quote N. Shenvi et al., because the quotation nicely describes the initial intention of my $\mathrm{PhD}$ thesis. Regarding the vibrational relaxation of $\mathrm{NO}$ scattering from $\mathrm{Au}(111)$ N. Shenvi et al. wrote: (2)

Perhaps most dramatically, we predict much larger vibrational energy loss for oriented NO beams in which the molecules are aligned to favor N-end approach of the molecule to the surface. It is our hope that these predictions will motivate new experiments to test the validity of our mechanism and ultimately to enhance our understanding of nonadiabatic processes at metal surfaces.

The first task to meet this challenge was to develop a method allowing for the orientation of the NO molecule.(3) Using this method, it was found, that the general trend predicted by theory considering the orientation (more vibrational relaxation for molecules oriented with the $\mathrm{N}$-atom towards the surface) proofed to be true.(4) However, it was also clear at an early stage of this work, that the existing theoretical models also make wrong predictions.

Because of that, the study of the NO vibrational relaxation on $\mathrm{Au}(111)$ was deepened by systematically changing not only the incidence orientation, but also the incidence translational energy and the incidence vibrational state of the impinging molecule. These experiments have been published in a series of experiments (5, 6, 7) and this thesis attempts to give a clear picture of the system under investigation by comparing results from these studies from various viewpoints.

Apart from the experiments on the $\mathrm{NO} / \mathrm{Au}(111)$ system, I have also performed some additional work during the course of my $\mathrm{PhD}$ thesis. In particular, I have contributed to initial experiments regarding the vibrational excitation of $\mathrm{CO}(v=0 \rightarrow 1)$ in collisions 


\section{Introduction}

with $\mathrm{Au}(111)$. That system was later studied in much more detail with another experimental setup in the same laboratory and the results on this topic are well described in publications(8, 9) and the Ph.D. thesis by Kai Golibrzuch(10). I thus decided not to cover this topic in this thesis.

Another project (covered in chapter 7) and my first publication (11) was about the generation of a molecular beam of highly vibrationally excited CO using perturbations. In that work, I prepared $\mathrm{CO}$ molecules in a high vibrational state $(v=17$ and 18) by means of optical pumping using three different laser pulses. The concept for this technique was developed in cooperation with professor Robert W. Field from the Massachusetts Institute of Technology in Cambridge (MA, USA) and is called pumppump-perturb-dump (P3D). 


\section{2}

\section{Theory and previous results}

In this section, I will provide some theoretical background from the field of surface dynamics and discuss some previous results relevant to this work. In addition, there is a section dealing with topics related to molecular spectroscopy, which I included as Appendix A.

\subsection{Introdution to electronically non-adiabatic processes}

\subsubsection{Theory and non-adiabatic effects at avoided curve crossings in the gas phase}

Modern understanding of chemistry is, often without that scientist are aware of it, based on the concept of the "adiabatic potential-energy surface" (PES), $\epsilon_{0}(\mathbf{R})$, see Fig. 2.1. The idea of a PES is that the energy of a molecular system $\epsilon_{0}(\mathbf{R})$ can be mapped out in terms of the nuclear coordinates (for example the bond lengths $R_{1}$ and $R_{2}$ in Fig. 2.1). For many systems, this representation is a reasonably well description. In that case, trajectories calculated on this PES - which determines the forces acting on the atoms/molecules — provide a clear picture how the atoms/molecules move as a function of time. Thereby it is assumed that, unless the system is electronically excited with radiation, the molecular system always stays on the PES of the electronic ground state. However, there can also be cases in which the electronic state changes non-radiatively during a dynamical event and such an event would be called an electronically non-adiabatic process.(12)

The term electronically non-adiabatic is a concept of quantum mechanics. When a 


\section{Theory and previous results}

process (e.g. a chemical reaction) is studied experimentally, it is thus far from trivial to answer the question, whether it followed an electronically adiabatic or non-adiabatic pathway. A process in which it is immediately clear, that the system must have undergone transitions to excited electronic states is chemi-luminescence: the emission of light not resulting from heat during a chemical reaction. The emission of light comes from a radiative decay from one electronic state to another. Thus the system must have left the electronic ground state in the first place. Well-known examples for chemiluminescence are the reactions in glow-sticks(13) or bio-luminescence, e.g. in fireflies such as the female glowworm Lampyris noctiluca.(14)

To answer the question under what conditions non-adiabatic transitions are likely to oc-

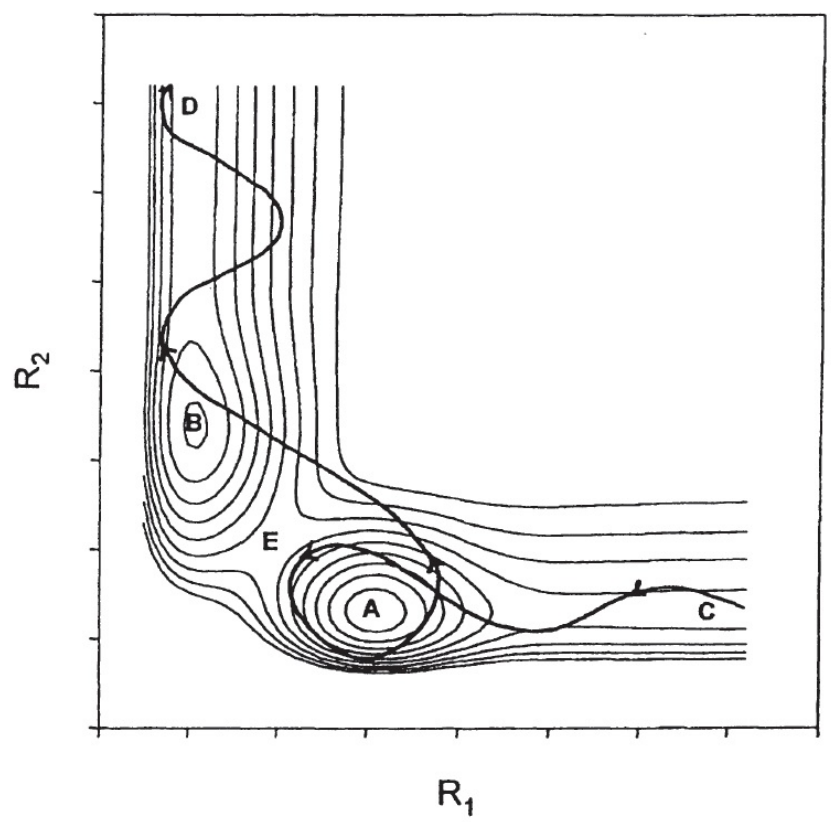

Figure 2.1: Potential energy surface - Schematic of a two-dimensional "potential energy surface" (PES). $R_{1}$ and $R_{2}$ are bond distances. The solid curves represent contours of equal energy $\epsilon_{0}(\mathbf{R})$. The PES determines the forces acting in the system and thus the trajectory (shown as thick black solid line with arrows on top). The PES is a concept based on the Born-Oppenheimer approximation. Whenever a system leaves the PES without being radiatively excited, this is considered an electronically non-adiabatic effect. From (15). Reprinted with permission from AAAS.

cur, it is necessary to consider theory more carefully. The non-relativistic Hamiltonian 
for a molecular system may be written as

$$
\hat{H}(\mathbf{r}, \mathbf{R})=\hat{T}_{\mathbf{R}}+\hat{H}_{\mathrm{el}}(\mathbf{r} ; \mathbf{R}),
$$

where $\mathbf{R}$ and $\mathbf{r}$ are the vectors of the nuclear and electronic coordinates, $\hat{T}_{\mathbf{R}}$ is the nuclear kinetic energy operator, and $\hat{H}_{\mathrm{el}}(\mathbf{r} ; \mathbf{R})$ it the electronic Hamiltonian. The semicolon in $\hat{H}_{\mathrm{el}}(\mathbf{r} ; \mathbf{R})$ is used to state, that the electronic Hamiltonian depends on $\mathbf{r}$ and only parametrically on $\mathbf{R}$. The electronic Hamiltonian contains the entire Hamiltonian of the system except for the nuclear kinetic energy operator. It includes the electronic kinetic energy operator and the Coulomb interactions. This means, that $\hat{H}_{\mathrm{el}}(\mathbf{r} ; \mathbf{R})$ can be considered the Hamiltonian for a system with non-moving nuclei, fixed at position R.

The exact solution $\Psi(\mathbf{r}, \mathbf{R})$ diagonalizes the full Hamiltonian $\hat{H}(\mathbf{r}, \mathbf{R})$

$$
\left\langle\Psi_{n}(\mathbf{r}, \mathbf{R})|\hat{H}(\mathbf{r}, \mathbf{R})| \Psi_{m}(\mathbf{r}, \mathbf{R})\right\rangle=\delta_{n m} E_{n} .
$$

However, the exact solution $\Psi(\mathbf{r}, \mathbf{R})$ is almost always unknown.

Instead, computational chemists diagonalize the electronic Hamiltonian $\hat{H}_{\mathrm{el}}(\mathbf{r} ; \mathbf{R})$ and the eigenfunctions $\phi_{m}(\mathbf{r} ; \mathbf{R})$ are called the adiabatic (Born-Oppenheimer) electronic wave functions

$$
\left\langle\phi_{k}(\mathbf{r} ; \mathbf{R})\left|\hat{H}_{\mathrm{el}}(\mathbf{r} ; \mathbf{R})\right| \phi_{j}(\mathbf{r} ; \mathbf{R})\right\rangle=\delta_{k j} \epsilon_{j}(\mathbf{R}),
$$

which are the solution of the electronic Schrödinger equation with the eigenvalues $\epsilon_{j}(\mathbf{R})$

$$
\hat{H}_{e l}(\mathbf{r} ; \mathbf{R})\left|\phi_{j}(\mathbf{r} ; \mathbf{R})\right\rangle=\epsilon_{j}(\mathbf{R})\left|\phi_{j}(\mathbf{r} ; \mathbf{R})\right\rangle
$$

The adiabatic PES corresponding to the electronic ground state is $\epsilon_{0}(\mathbf{R})$, the electronically exited states are $\epsilon_{j}(\mathbf{R})$ with $j \geq 1$. The exact wave function $\Psi(\mathbf{R}, \mathbf{r})$ can now be expanded in terms of $\phi_{j}(\mathbf{r} ; \mathbf{R})$ with the ansatz

$$
\Psi_{n}(\mathbf{r}, \mathbf{R})=\sum_{i} \phi_{i}(\mathbf{r} ; \mathbf{R}) \Omega_{i}(\mathbf{R})
$$

Substituting Eq. 2.5 into the Schroedinger equation for the full Hamiltonian from Eq. 2.1, we obtain (12, 15, 16)

$$
\left[\hat{T}_{\mathbf{R}}+\epsilon_{j}(\mathbf{R})-E\right] \Omega_{j}(\mathbf{R})=-\sum_{i \neq j}\left[\hat{T}_{i j}^{(1)}(\mathbf{R})+\hat{T}_{i j}^{(2)}(\mathbf{R})\right] \Omega_{j}(\mathbf{R})
$$




\section{Theory and previous results}

where

$$
\begin{aligned}
& \hat{T}_{i j}^{(1)}=\frac{-\hbar^{2}}{2 \mu}\left\langle\phi_{i}\left|\nabla_{R}\right| \phi_{j}\right\rangle \cdot \nabla_{R} \\
& \hat{T}_{i j}^{(2)}=\frac{-\hbar^{2}}{2 \mu}\left\langle\phi_{i}\left|\nabla_{R}^{2}\right| \phi_{j}\right\rangle
\end{aligned}
$$

are the first and second order non-adiabatic coupling terms, which are also called momentum and kinetic energy non-adiabatic coupling terms, respectively. In these equations, $\mu$ is the reduced mass, and $\nabla_{R}$ is the nuclear gradient operator. If the right term of equation 2.6 is neglected - meaning no coupling between different electronic states -, we obtain

$$
\left[\hat{T}_{\mathbf{R}}+\epsilon_{j}(\mathbf{R})-E\right] \Omega_{j}(\mathbf{R})=0
$$

Equation 2.9 means that - in case the non-adiabatic coupling terms are neglected - the nuclear motion is governed by a Schroedinger equation with a potential energy $\epsilon_{0}(\mathbf{R})$ that is the solution of the electronic Schroedinger equation 2.4. It is thus possible to first compute the electronic structure part 2.4 for fixed nuclei and then the nuclear dynamics part 2.9. This is known as the Born-Oppenheimer approximation.(16)

When does the approximation fail? The answer can be found in the equations for the non-adiabatic coupling terms. These become large, when 1) the first or second derivative of the adiabatic wave functions with the nuclear coordinates are large, meaning simply that a potential has a strong slope or curvature. In addition, the integral is large when 2) the adiabatic wave functions $\phi_{i}$ and $\phi_{j}$ are close in energy.

There is an extensive literature on the breakdown of the Born-Oppenheimer approximation in gas-phase atomic and molecular collisions (see (17, 18) and references therein). The most common examples involve systems with multiple potential energy surfaces which classically would cross. If the symmetry of the curves is the same, the crossing is forbidden by quantum mechanics, and interactions will prevent an actual crossing. The situation is particularly simple if we are dealing with atom-atom collisions, in which case the potential energy surfaces are one-dimensional potential energy curves.

Consider the case of a charge changing collision where two neutral atoms colloid and an ion pair is formed, e.g. $\mathrm{Na}+\mathrm{I} \rightarrow \mathrm{Na}^{+}+\mathrm{I}^{-}$. At large distance, the ionic curve is above the neutral curve by

$$
\Delta E=I P-E_{\mathrm{A}}
$$


where $I P$ is the ionization potential of the alkali atom and $E_{\mathrm{A}}$ is the electron affinity of the halogen. The ionic and covalent curves which interact to form an ion-pair are both ${ }^{1} \Sigma^{+}$states. Since they are of the same symmetry, there will be an avoided curve crossing at $R_{\mathrm{C}}=1 / \Delta E$. As the neutral $\mathrm{Na}$ and I atoms approach they proceed on the neutral curve until they reach a distance of $R_{\mathrm{C}}$. At that point they can proceed adiabatically and move on to the ionic curve or non-adiabatically and stay on the neutral curve. After reaching the inner turning point of the collision, the two atoms will again cross the avoided crossing at $R_{\mathrm{C}}$ with the possibility of making and adiabatic or non-adiabtic crossing. Ion pairs result if the two crossings are adiabatic-non-adibatic or non-adiabatic-adiabatic and the probability of forming an ion pair is given by

$$
P_{\text {ion-pair }}=2 p(1-p)
$$

where $p$ is the probability of making a non-adiabatic transition at the crossing point. An approximate expression for $\mathrm{p}$ was derived by Landau, Zener, and Stueckelberg(19, 20, 21, 22)

$$
p=\frac{\frac{2 \pi}{\hbar} H_{12}^{2}}{d / d R\left|V_{\text {ion }}-V_{\text {neut }}\right| v_{R}}
$$

where $H_{12}$ is the coupling matrix element (splitting) between the neutral and ionic curves, $V_{\text {ion }}$ and $V_{\text {neut }}$ are the ionic and neutral potential curves, $v_{\mathrm{R}}$ is the radial velocity. There is a large body of measurements of charge transfer collisions that are in good accord with the Landau-Zener-Stueckelberg theory.(18, 23)

Pioneering work demonstrating non-adiabatic effects at avoided curve crossings in realtime was performed by A. Zewail and co-workers on alkali halides such as sodium iodide, see Fig. 2.2.(24) The adiabatic electronic ground state of the molecule has ionic $\left(\mathrm{Na}^{+}\right.$ $+\mathrm{I}^{-}$) character near its equilibrium bond length, and covalent (Na-I) character upon dissociation. The situation can be described with two diabatic ${ }^{1}$ potentials (one ionic and one covalent, shown as solid lines in Fig. 2.2 that cross a bond length of $6.93 \AA$. In the adiabatic view (dashed lines), these potentials do not cross (non-crossing rule). In the experiment, molecules are excited to the first electronically excited adiabatic state. This is done with a femtosecond laser pulse, that excites to a superposition of different vibrational states. This superposition can be described by a wave packet,

\footnotetext{
${ }^{1}$ The term diabatic means that nuclear kinetic energy operator $\hat{T}_{\mathbf{R}}$ is diagonalized (instead of the electronic Hamiltonian in the adiabatic case $):\left\langle\phi_{k}^{\prime}(\mathbf{r} ; \mathbf{R})\left|\hat{T}_{\mathbf{R}}\right| \phi_{j}^{\prime}(\mathbf{r} ; \mathbf{R})\right\rangle=\delta_{k j} \epsilon_{j}(\mathbf{R})$.
} 

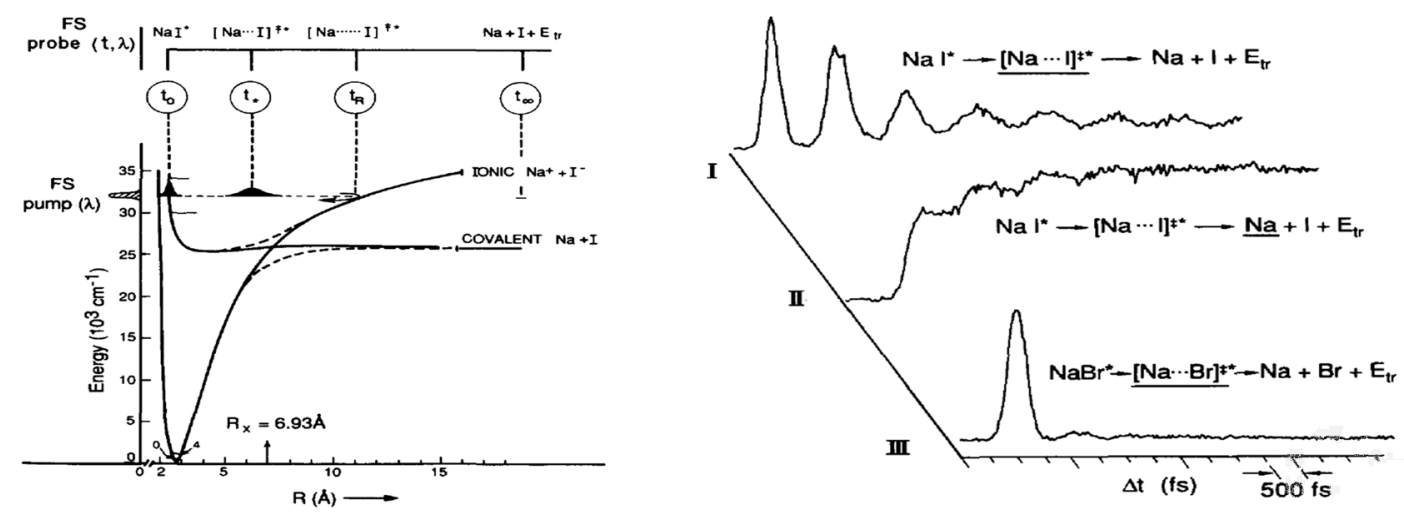

Figure 2.2: Non-adiabatic transition at a curve crossing in the gas-phase - left: Potential energy curves and motion of the wave packet for the NaI molecule. At the top of the figure, the different probed configurations are given. right: Temporal population of the configurations as shown of the left side. Reprinted with permission from (24). Copyright 1988, AIP Publishing LLC.

travelling back and forth in the excited state with an oscillation period of approximately 1.25 ps. Every time the wavepacket travels back and forth, some molecules undergo a non-adiabatic transition to the adiabatic ground state. This population transfer can be detected with transient femtosecond spectroscopy.

The curve crossings at alkali halides are still part of modern research, for example the photo-dissociation of alkali-halides is studied in helium nano-droplets (25) and the non-adiabatic transition probability can be tuned with Stark pulses (26). An example for a non-adiabatic transition in the gas-phase regarding the vibrational dynamics of the NO molecule has recently been found for the vibrational relaxation in the collsion system $\mathrm{NO}\left(X^{2} \Pi(v=1)\right)+\mathrm{Ar} \rightarrow \mathrm{NO}\left(X^{2} \Pi(v=1)\right)+$ Ar, which was attributed to a curve crossing between the $\left(\mathrm{A}^{\prime \prime}, v=1\right)$ and $\left(\mathrm{A}^{\prime}, v=0\right)$ vibronic states of the collision system.(27)

\subsubsection{Non-adiabatic effects at metal surfaces}

So far it was shown that the Born-Oppenheimer approximation works reasonably well as long as electronic states are well separated in energy and that non-adiabatic transitions occur at avoided curve crossings. The situation in the dynamics at metal surfaces is more complicated, because metals have a continuum of electronic states and electronhole pairs can be excited in the metal. This means that - speaking in terminology of 
dynamics in the gas-phase - there is an infinitesimal amount of "curve crossings", see left panel of Fig. 2.3. (28) Already this simple theoretical consideration suggests, that non-adiabatic processes are important for molecules at metal surfaces. An explanation of how electron-hole pair excitation can occur in a molecule-surface interaction is given in the right panel of Fig. 2.3. Indeed, there is continuously growing evidence
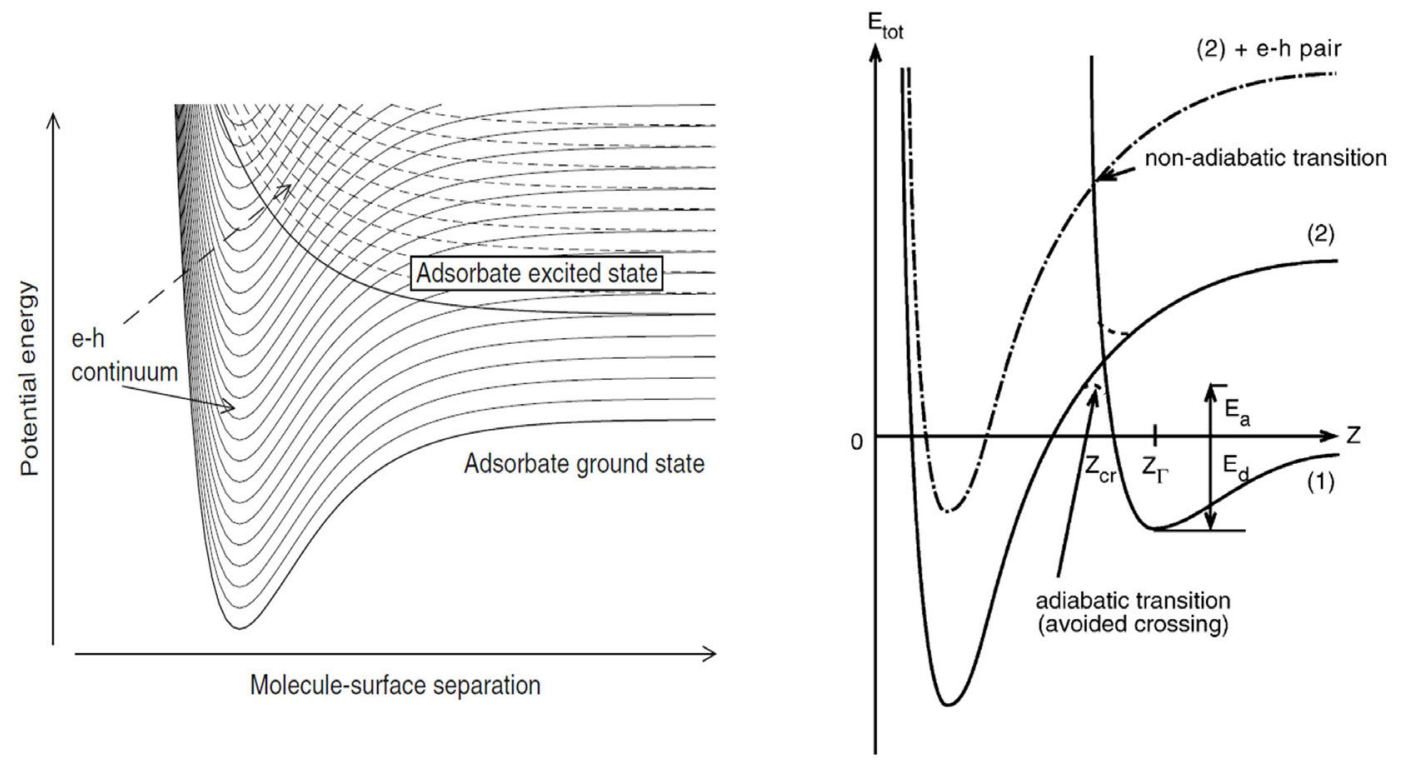

Figure 2.3: The importance of electron-hole pairs for non-adiabatic effects at metal surface - (left) The interaction of moleucles with electron-hole pairs at metal surfaces leads to a continuum of electronically excited states. This leads to an infinite amount of curve crossings. Fig. from Ref. (28),p.279. (right) Example of how eletron-hole pairs can be generated in a molecule-metal collision. The figure shows the energetics of a system with a precursor state (1) with a binding energy $E_{\mathrm{d}}$ at $Z_{\mathrm{r}}$ and a second state (2) that can be accessed via an activation barrier $E_{\mathrm{a}}+E_{\mathrm{d}}$ at $Z_{\mathrm{cr}}$. In case the transition from (1) to (2) occurs adiabatically, the system follows the dashed line (avoided crossing). However, in case the electronic structure cannot follow the sudden change of the nuclear motion, the system follows (1) beyond the crossing point and then makes a transition to the dashed dotted PES. This way electron-pairs with different energies can be excited. Fig. from Ref. (29).

for electronically non-adiabatic effects at metal surfaces. The interested reader may be referred to several review articles and the references therein.(29, 30, 31, 32, 33) Results for two examples out of many, where electron-hole pair excitation is thought to be involved in the dynamics on metal surfaces are shown in Fig. 2.4. Panel (A) of Fig. 2.4 


\section{Theory and previous results}

shows results for the chemi-current that can be measured upon adsorption of various atoms or molecules on $\mathrm{Ag} / \mathrm{Si}$ Schottky diodes. The adsorption process leads to the excitation of electron-hole pairs which can be detected as a current. It is found that all tested adsorption reactions lead to energy dissipation into electron-hole pairs. The probability of exciting detectable electron-hole pairs with an energy above the Schottky barrier of 0.2 to $0.3 \mathrm{eV}$ increases linearly with the adsorption energy.(34)

A very recent and important result deals with the interaction of hydrogen atoms with
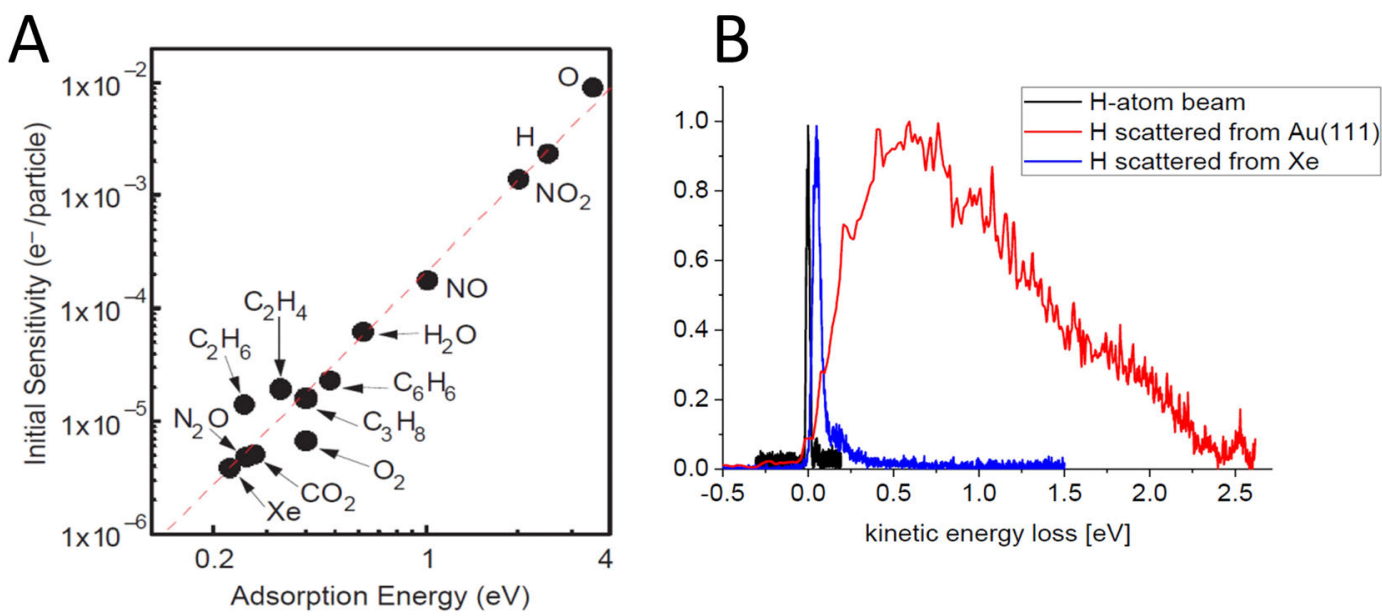

Figure 2.4: Two experiments clearly showing the importance of electron-hole pairs in surface dynamics on metals - (A) The absorption of various atoms or molecules on $\mathrm{Ag} / \mathrm{Si}$ Schottky diodes excites electron hole-pairs. These can be detected as chemi-currents. The probability of exciting a detectable electron increases linearly with the adsorption energy. From (34). Reprinted with permission from AAAS. (B) When Hatoms are scattered from $\mathrm{Au}(111)$ energy is efficiently lost to electronic excitations in the metal and the $\mathrm{H}$-atoms efficiently loose translational energy to the surface. The measurement was performed at an incidence translational energy of $2.72 \mathrm{eV}$ and an incidence angle of $-45^{\circ}$. Scattered molecules were detected at an angle of $45^{\circ}$. Figure from Ref. (35). Reprinted with permission from O. Bünermann.

a $\mathrm{Au}(111)$ surface, see panel (B) of Fig. 2.4. In the experiment, hydrogen atoms with a well defined incidence translational energy of $2.72 \mathrm{eV}$ (black line) were scattered from a $\mathrm{Au}(111)$ surface (red, solid line) as well as from a Xenon-covered Au surface (blue line). After the collsion, the kinetic energy distributions of surface scattered molecules was measured. The Xenon-covered surface can only take up energy in form of phonons. As the coupling between these phonons and the $\mathrm{H}$-atoms translation is rather weak, the 
$\mathrm{H}$-atoms are scattered back with only little loss of translational energy. For H-atoms scattered from the clean $\mathrm{Au}(111)$ surface, energy can also be non-adiabatically transferred to electron-hole pairs. This coupling seems to be very strong, and the scattered $\mathrm{H}$-atoms loose a lot of their translational energy to the surface.(35) This experiment might also explain the high sticking probability of $\mathrm{H}$-atoms on many metal surfaces. The two experiments discussed up to this point show, that the generation of electronhole pairs for reactions at metal surfaces are the rule rather than the exception and that non-adiabatic effects can play an important role for chemical reactivity.

\subsection{Electron transfer energetics at metal surfaces}

The result that electron-hole pairs are important in surface dynamics and are generated by non-adiabatic transitions poses the question: What is the nature of these nonadiabatic transitions? In other words: Between which potentials do the non-adiabatic transitions occur?

I have already discussed the gas-phase example of the avoided curve-crossing in the $\mathrm{NaI}$ molecule (Fig. 2.2). In this example, the non-adiabatic transitions were due to electron transfer. Upon vibration of the molecule, the adiabatic potentials rapidly change from a covalent (NaI) character to an ionic character. The inability of the electronic structure to adapt to the change of internuclear separation induced non-adiabatic transitions. Indeed, it is believed that electron transfer also plays an important role for many nonadiabatic effects in gas-metal surface interactions.(29, 32) A characteristic feature of metal surfaces is that they can be directly involved in electron transfer reactions and that they stabilize ions in their vicinity due to the image-charge effect (28). This stabilization of ionic species can lead to additional curve crossings to the potential energy landscape.

In order to obtain an estimate in which systems of gas-metal surface dynamics electron transfer might be important, it is useful to study some theory on the energetics of electron transfer at metal surfaces. A positive point charge $q$ at a distance $z$ in front of a metal surface induces a polarization cloud of opposite charge on the surface (Fig. 2.5 A). According to the method of image charges in electrostatics, the field lines outside the metal can be described as if the positive charge was interacting with its negative image charge $-q$ at distance $-z$ inside the surface (Fig. 2.5B). 


\section{Theory and previous results}

A

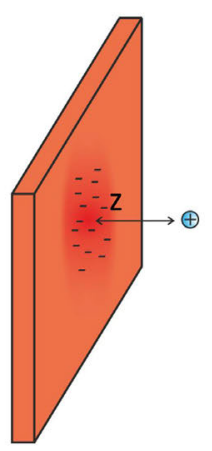

B

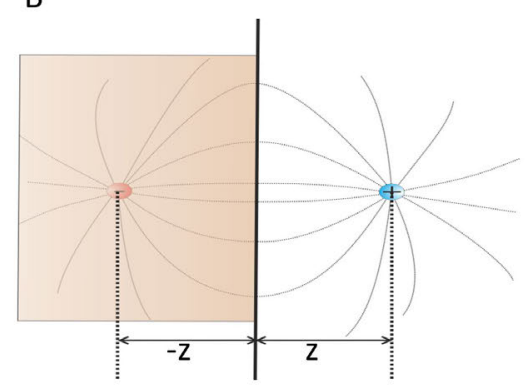

C

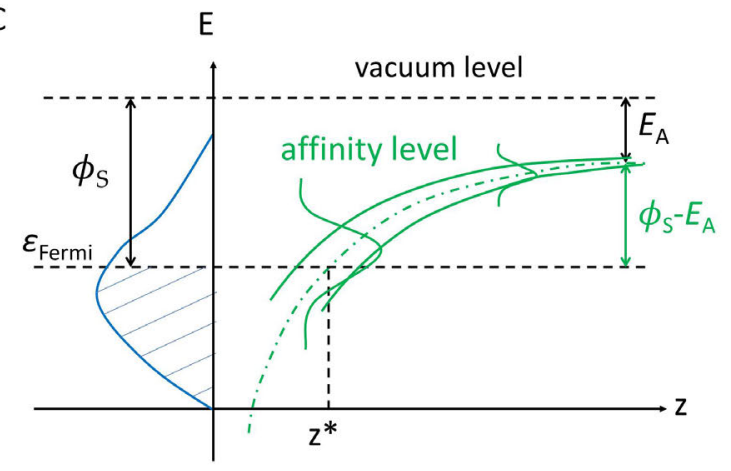

D
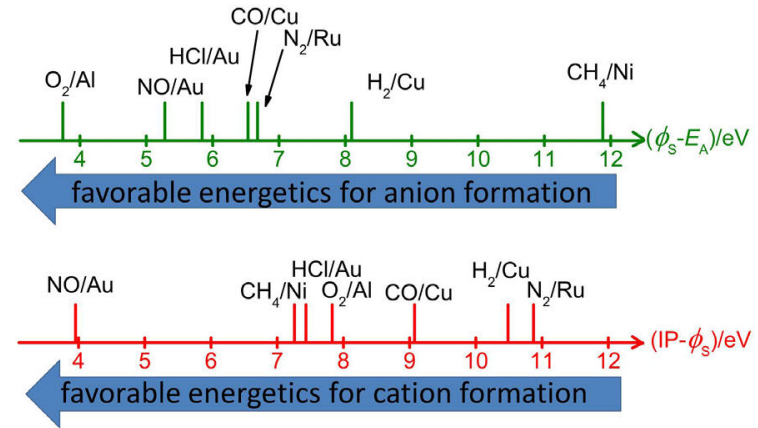

Figure 2.5: Electron transfer energetics at metal surfaces - (A) A positive charge (blue) at a distance $z$ from a metal surface induces negative (red) surface charge. (B) The electric field lines outside the metal are as if a negative charge was placed at position $-z$. (C) For a molecule far from a metal surface, the energy required to transfer an electron from the metal to the molecule (the formation of an anion) is given by the difference of the surface work function $\phi_{S}$ and the electron affinity of the molecule $E_{A}$. This difference has to be overcome by image charge stabilization to make electron transfer (ET) feasible. As the affinity level is stabilized upon approach of the molecule to the surface, the affinity level broadens as the lifetime decreases. The blue function indicates the density of states in the metal. (D) The energetics for possible ET reactions are compared for different systems. The smaller the value $\phi_{\mathrm{S}}-E_{\mathrm{A}}$ the more likely is the anion formation. In a similar way, one can argue that cation formation is energetically feasible for small values of a difference between the first ionization potential $I P$ and the surface work function $\phi_{S}$. The $\mathrm{NO} / \mathrm{Au}(111)$ system energetically allows for anion as well as for cation formation. Values for $\phi$ are for face centered cubic (111) surfaces from Ref. (36). Values for $I P$ are experimental values from NIST webbook of chemistry(37). The $E_{\mathrm{A}}$ values are computational values (composite Gaussian-4 theory) (37), except for the $E_{\mathrm{A}}$ of methane, which is taken from Ref. (38). 
To a fist approximation the interaction between the charge and its image charge can be described by a Coulomb interaction. In a more detailed study(39), Appelbaum and Hamann showed that the energy of a point charge $q$ at a distance $z$ greater than $2 \AA$ from a surface (more exactly the distance from the jellium edge) is given by

$$
E(z)=-\frac{1}{4 \pi \epsilon_{0}} \frac{q^{2}}{4(z-d)}
$$

where $\epsilon_{0}$ is the vacuum permittivity, and $d$ is an origin shift, which is a function of the electron density at the metal surface.

The image charge effect promotes electron transfer between a metal surface and an approaching atom or molecule (Fig. 2.5 C). The energy released when an electron from the vacuum is added to a neutral atom or molecule is the electron affinity $E_{\mathrm{A}}$.

$$
X+e^{-} \rightarrow X^{-}+E_{\mathrm{A}}
$$

The energy that is required to remove an electron from the Fermi level at the Fermi energy $\left(\epsilon_{\text {Fermi }}\right)$ is the surface work function $\phi_{S}$. This means, that the energy required to transfer an electron from the surface to an atom/molecule far from the surface is given by the difference $\phi_{S}-E_{A}$. However, the electron affinity level is energetically stabilized upon approach of the molecule. Eventually, the affinity level might cross the Fermi energy at a certain critical distance $z^{*}$ and electron transfer from the metal to the molecule becomes energetically possible. The stabilization due to image charge effect is limited due to quantum effects (formula 2.12 is only a good approximation for $z>2 \AA$ ), and the electron transfer energetics are more complex than the pure image charge effect. Nevertheless, values of $\phi_{S}-E_{A}$ can be used as a predictor whether electron transfer (anion formation) is likely to be involved in a particular gas-surface interaction (Fig. 2.5D).(40) The $\mathrm{NO} / \mathrm{Au}(111)$ system studied in this work has favorable energetics for anion formation due to the large electron affinity of $\mathrm{NO}(+0.03 \mathrm{eV})$ compared to other diatomic molecules, the work function of $\mathrm{Au}(111)$ is $5.31 \mathrm{eV}$. The image charge effect does also stabilize cations in front of a surface. In this case, the relevant measure for the energetics is the difference of the first ionization potential (IP)

\footnotetext{
${ }^{1}$ Values for the electron affinity are typically negative for diatomic molecules, e.g. $-3.155 \mathrm{eV}$ for $\mathrm{H}_{2-},-1.967 \mathrm{eV}$ for $\mathrm{N}_{2}$ or $-0.531 \mathrm{eV}$ for $\mathrm{HCl}$. The values $\phi_{\mathrm{S}}$ of the surface work function are relatively similar for the different metals, ranging from $4.24 \mathrm{eV}$ for $\mathrm{Al}(111)$ to $5.7 \mathrm{eV}$ for $\mathrm{Pt}(111)$. See the caption of Fig. 2.5 for references.
} 


\section{Theory and previous results}

and the surface work function $\phi_{\mathrm{S}}$. Although the energetics would seem not to rule it out, to my knowledge, there are no known examples of ET at surfaces involving cation formation. With respect to this work, it should be noted, that the stretched bonds of highly vibrationally excited NO molecules favor anion formation. I will return to this point in section 2.4 .2 .

\subsection{Vibrational dynamics at metal surfaces}

In this section I summarize previous results for the vibrational dynamics at metal surfaces relevant to this work.

\subsubsection{Vibrational dynamics of adsorbed molecules}

A lot of our understanding of how a molecules vibration couples to surface degrees of freedom comes from the study of molecules adsorbed on surfaces. Vibrational dynamics of adsorbates have been heavily studied with spectroscopic methods like reflectionadsorption IR spectroscopy (RAIRS), surface-enhanced Raman spectroscopy (SERS), sum-frequency generation spectroscopy (SFG), two-photon photoemission spectroscopy (2PPE), high resolution electron energy loss spectroscopy (HREELS) or X-RAY spectroscopy. Many of these methods can also be used for realtime measurements using pump-probe techniques. See Ref.(41) for a recent review on the topic.

Important to this work is that the adsorbates vibration can couple either to phonons or (for metal or semi-conductor surfaces) to electronic excitations in the metal. While the coupling to phonons is an adiabatic process, the coupling to electron-hole pairs is a non-adiabatic process. For d-band metals, the coupling of the adsorbates vibration to electrons $1 / \tau_{\mathrm{el}}$ is found to be very fast ( $\tau_{\mathrm{el}}$ is on the order of 0.1 to $1 \mathrm{ps}$ ) compared to the coupling of the vibration to phonons $\left(\tau_{\mathrm{ph}} \geq 1 \mathrm{ps}\right)$, see Fig. 2.6. The coupling between a molecules vibration to phonons on a nearby solid surface $k_{p h}=1 / \tau_{p h}$ can be estimated with models such as Silbey's phonon relaxation model(44). This classical model treats the vibration to phonon coupling as a radiative energy transfer between an oscillating dipole moment (the molecule) and a receiver (the surface) which is characterized by its complex dielectric constant. Within this model, $k_{p h}$ is proportional to $1 / \tilde{\nu}^{3}$, meaning that vibration to phonon coupling decreases rapidly for large vibrational frequencies $\tilde{\nu}$. This is also supported by the consideration that a high frequency stretching mode (e.g. 


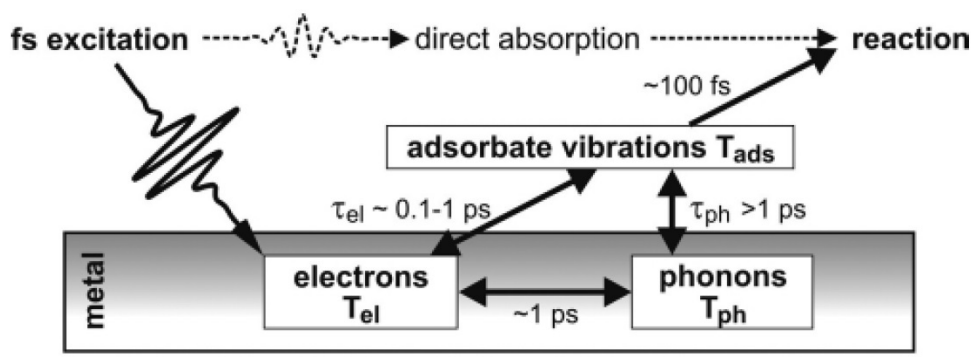

Figure 2.6: Processes and associated timescale for adsorbates on metal surfaces - The vibrational motion of an adsorbate is coupled to electrons (electron-hole pairs) with the time constant $\tau_{\mathrm{el}}$ and to phonons $\tau_{\mathrm{ph}}$. The adsorbates vibration can trigger chemical reactions, but also other processes such as surface diffusion(42). The energy dissipation or reactions can be probed by exciting the vibrational modes of the adsorbates. However, it is also possible to use intense fs-laser rediation to create a hot electron distribution in the metal. The electron distribution is then described with a temperature $T_{\mathrm{el}}$ which is different from the phonon temperature $T_{\mathrm{ph}}$. These experiments also provide insight into the relative rates of energy transfer. Figure from Ref. (43).

$2078 \mathrm{~cm}^{-1}$ for the $\mathrm{C}-\mathrm{O}$ stretch in the $\mathrm{CO}$ molecule) would have to simultaneously excite several low frequency phonon modes typically available on metals $\left(\leq 200 \mathrm{~cm}^{-1}\right)$ to allow for energy transfer. This means that vibration to phonon coupling is unlikely for systems with a large frequency mismatch between these modes. For example, the life time of the $\mathrm{CO}(v=1)$ stretching mode on $\mathrm{Cu}(100)$ was found to be $\tau=2 \pm 1 \mathrm{ps}$ (main channel of energy transfer is via $\left.\tau_{\mathrm{el}}\right)$.(45) In contrast to that, $\mathrm{CO}(v=1)$ adsorbed on the insulator $\mathrm{NaCl}(100)$ has a vibrational lifetime of $\tau=4.3 \mathrm{~ms}$.(46) This difference in lifetime of more than by a factor of $10^{9}$ is largely due to the fact, that $\mathrm{NaCl}$ does not offer the possibility of taking up vibrational energy as electronic excitations.

By now, all four vibrational modes of $\mathrm{CO}$ on $\mathrm{Cu}(100)$ have been studied. These are the internal $\mathrm{C}-\mathrm{O}$ stretch vibration $\left(2078 \mathrm{~cm}^{-1}\right)$, the perpendicular frustrated translation $\left(345 \mathrm{~cm}^{-1}\right)$, the frustrated rotation $\left(288 \mathrm{~cm}^{-1}\right)$ and the parallel frustrated translation $\left(32 \mathrm{~cm}^{-1}\right)$. Modern calculations, using methods such as molecular dynamics with electronic friction (MDEF), are capable of accurately calculating the non-adiabatic couplings for the electron-hole pair induced vibrational energy transfer and the modespecific lifetimes of diatomic molecules on different metal surfaces on an ab initio level.(47, 48) A physical explanation of the relaxation process is that the relaxation results from a damping of the vibrational mode due to electronic friction. When the 


\section{Theory and previous results}

$\mathrm{C}-\mathrm{O}$ bond is streched during the vibration, the anti-bonding $2 \pi^{*}$ level is lowered in energy allowing for charge flow from the metal in that molecular orbital. This charge flows back after the bond compression. As this nuclear motion is too fast for the electrons to follow this motion adiabatically, there is "electronic friction". This leads to a damping (damped oscillating dipole moment) of the vibration and the generation of electron-hole pairs.(41, 49)

\subsubsection{Adiabatic versus non-adiabatic vibrational dynamics in gas- surface collisions}

The vibrational dynamics of molecules scattering from metal surfaces are more complex than for adsorbed molecules. For example, the dynamics depend on details of the scattering conditions such as the molecular orientation or the position where the molecule approaches the surface (e.g. top or hollow sites). In addition, the molecule has additional degrees of freedom such as translational and rotational excitation, which leads to additional channels for energy transfer. Nevertheless, there are examples in which adiabatic and non-adiabatic vibrational dynamics could be clearly distinguished. I want to explain this on the example of the vibrational excitation in gas-surface collisions in two systems: the scattering of $\mathrm{NH}_{3}$ from $\mathrm{Au}(111)$ and of $\mathrm{NO}$ from $\mathrm{Ag}(111)$, see Fig. 2.7 .

Kay et al. studied the vibrational excitation of the umbrella mode in $\mathrm{NH}_{3}$, which becomes excited when $\mathrm{NH}_{3}$ in its vibrational ground state is scattered from $\mathrm{Au}(111)$. It was found, that the vibrational excitation probability is largely independent of the surface temperature. Instead, the vibrational excitation sets in when the incidence translational energy exceeds a threshold corresponding to the vibrational spacing in $\mathrm{NH}_{3}$ (Fig. 2.7D). A further increase of translational energy leads to a linear increase of the excitation probability. The interpretation of this experiment was, that the collision with the surface induces an energy transfer between translational energy (prior to the collision) and vibrational energy (after the collision). This energy transfer can be explained without the involvement of electron-hole pair excitation and is believed to be the result of an adiabatic, mechanical excitation mechanism.(50) As opposed to this, the vibrational excitation of NO scattering from $\operatorname{Ag}(111)$ is believed to result from an electronically non-adiabatic process. In this case, the vibrational excitation 

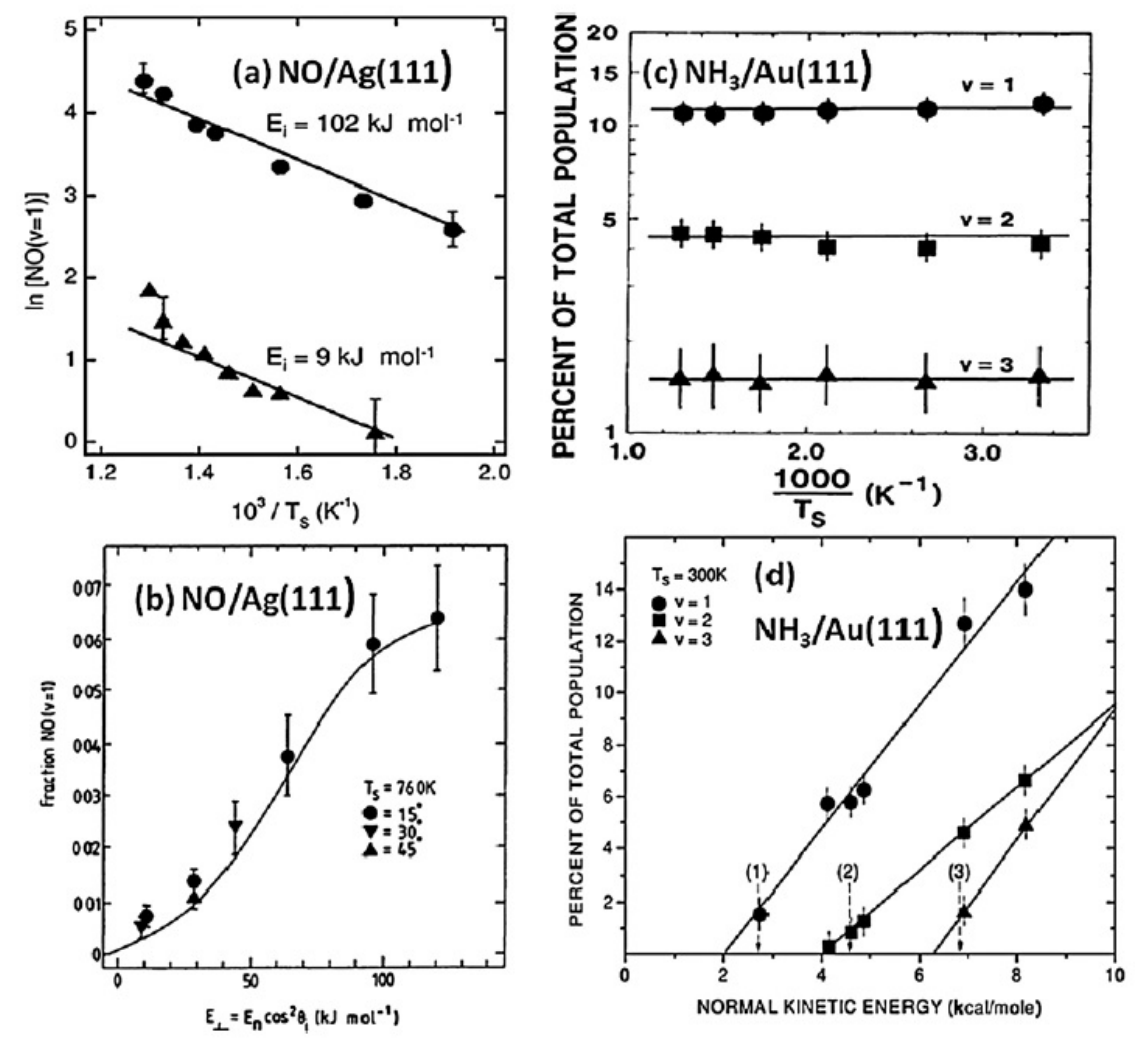

Figure 2.7: Adiabatic vs non-adiabatic vibrational excitation in gas-surface collisions - The vibrational excitation of NO scattering from $\mathrm{Ag}(111)$ (panels (a) and (b)) proceeds via a non-adiabatic mechanism in which energy of thermally excited electron-hole pairs in converted into vibration of the molecule. The process is thus strongly temperature dependent.(51) As opposed to this, the excitation of the $\nu_{2}$ umbrella mode in $\mathrm{NH}_{3}$ in collisions with $\mathrm{Ag}(111)$ results from an adiabatic mechanism and the excitation energy comes from the molecules incident translational energy (panels (c) and (d)).(50) This leads to translational thresholds in the excitation probalilties, corresponding to the uptake of vibrational energy. Reproduced from Ref. (52) with permission of The Royal Society of Chemistry. 


\section{Theory and previous results}

excitation probability was found to increase exponentially with the surface temperature $T_{S}$. This could be well described by an Arrhenius function with an activation energy of the vibrational spacing. This $T_{\mathrm{S}}$-dependence is believed to be the result of a vibrational excitation mechanism that requires thermally excited electron-hole pairs. In this case, the vibrational excitation probability was still found to increase with the incidence translation, but no threshold behaviour was observed.(51) The examples are discussed in more detail in Ref. (52).

\subsubsection{Vibrational dynamics of NO scattering from $\mathrm{Au}(111)$}

Cooper et al. measured the excitation probability of NO $v=0 \rightarrow 1$ and $v=0 \rightarrow 2$ upon collision with $\mathrm{Au}(111)$ as function of the incidence translational energy and surface temperature, see Fig. 2.8. (53) The results are very similar to those of NO scattering
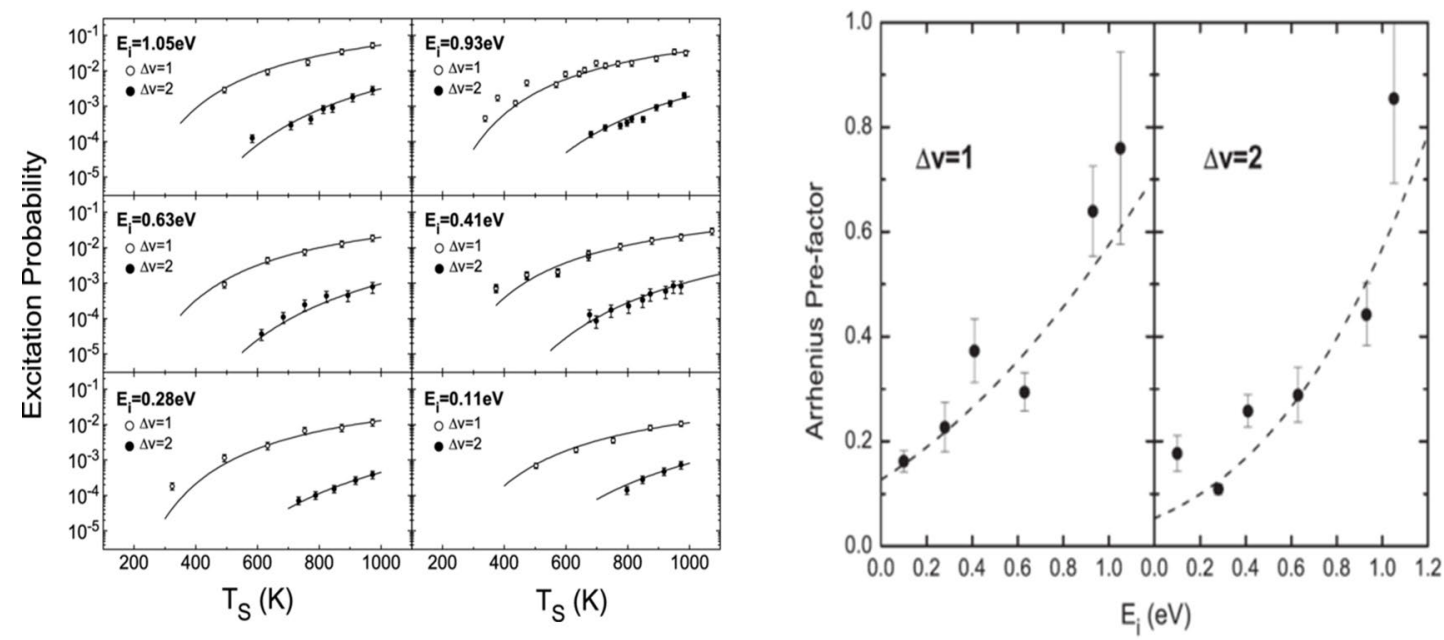

Figure 2.8: Vibrational excitation for NO scattering from $\mathrm{Au}(111)$ - Left: Measured vibrational excitation probability of $\mathrm{NO} v=0 \rightarrow 1$ and $v=0 \rightarrow 2$ (symbols) as function of surface temperature $T_{\mathrm{S}}$ and incidence translational energy $E_{\mathrm{i}}$. The excitation probabilites $P_{01}$ and $P_{02}$ are fitted with Arrhenius functions (solid lines) of the functional form $P_{01}=A_{01} \cdot \exp ^{-\frac{0.236 \mathrm{eV}}{k_{\mathrm{B}} T_{\mathrm{S}}}}$ and $P_{01}=A_{01} \cdot \exp ^{-\frac{0.476 \mathrm{eV}}{k_{\mathrm{B}} T_{\mathrm{S}}}}$, respectively. The vibrational excitation probabilities increase strongly with the surface temperature $T_{S}$ and also with incidence translation. Right: The Arrhenius pre-factors $A_{01}$ and $A_{02}$ are plotted as function of the incidence translation. Reprinted with permission from (53). Copyright 2012, AIP Publishing LLC.

from $\operatorname{Ag}(111)$, with clear signatures of an electronically non-adiabatic excitation mechanism. The vibrational excitation probability was found to increase exponentially with 
the surface temperature $T_{S}$. This could be well described by an Arrhenius function with an activation energy of the vibrational spacing $(0.236 \mathrm{eV}$ for $v=0 \rightarrow 1$ and $0.476 \mathrm{eV}$ for $v=0 \rightarrow 2$ ). It was also found, that the vibrational excitation probability increases with incidence translational energy. In the Arrhenius description this was modeled by an Arrhenius-prefactor that depends on the non-adiabatic coupling strength which in turn depends on the translational energy. The vibrational excitation experiments have recently been extended to the NO $v=0 \rightarrow 3$ excitation channel and compared to theoretical calculations.(10, 54)

The vibrational relaxation of vibrationally excited NO scattering from $\mathrm{Au}(111)$ has been measured by Huang et al. for the incidence vibrational state $v_{\mathrm{i}}=2$ (55) and recently by Golibrzuch et al. for $v_{\mathrm{i}}=3$ (56): These vibrational states can be accessed via overtone pumping with infrared laser radiation. Due to the large background of molecules in $v=0$ in these experiments, it was not possible to evaluate absolute relaxation probabilities. Thus, the results are described in terms of branching ratios, that indicate the relative population among the scattered vibrational states, see Fig. 2.9. In contrast to the vibrational excitation, the vibrational relaxation is only weakly dependent on the surface temperature (see the branching ratio of $v=1 / v=2$ (relaxation) on the left panel in Fig. 2.9p . Although both processes are believed to be the result of a non-adiabatic energy transfer, the temperature is rather unimportant for the relaxation, because an energy transfer to electrons does not require them to be thermally excited. The relaxation was found to be strongly influenced by the translational energy. For $v_{\mathrm{i}}=3$, the fraction of molecules scattered in the vibrational elastic channels $(v=3 \rightarrow 3)$ compared to the vibrational inelastic channels $(v=3 \rightarrow 2$ and $v=3 \rightarrow 1)$ was found to decrease from 0.8 at $0.1 \mathrm{eV}$ incidence translational energy to 0.4 at $1.0 \mathrm{eV}$. This was attributed to an increased coupling between the molecular vibration to surface electrons at high incidence translational energy, as the NO molecule penetrates closer into the electron cloud.

The observation of the very efficient coupling of the vibrational motion in the NO molecule to electron-hole pairs ( $\mathrm{e}-\mathrm{h}$ pairs) in the metal has triggered the desire to prove that the $\mathrm{e}-\mathrm{h}$ pair excitation occurs more directly. In principle, this could be done using chemi-current detection, e.g. with Schottky diodes (compare Fig. 2.4), 


\section{Theory and previous results}
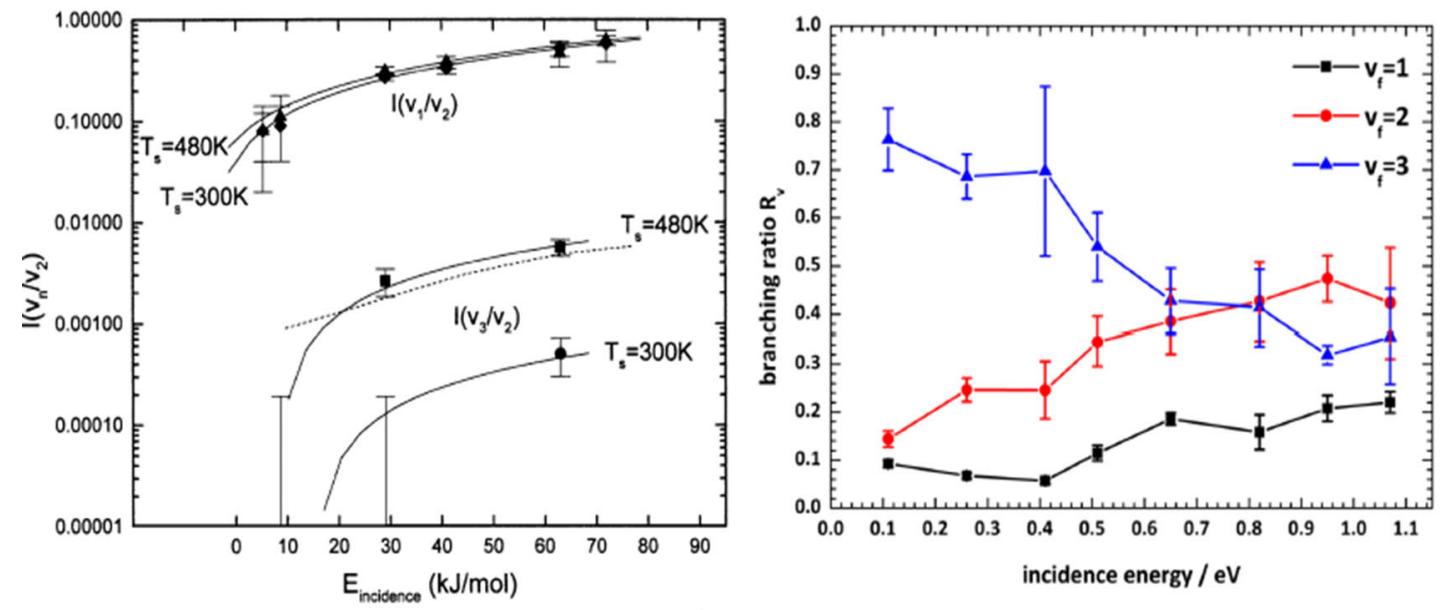

Figure 2.9: Vibrational relaxation of NO $(v=2$ and $v=3)$ scattering from $\mathbf{A u}(\mathbf{1 1 1})$ - Left: Branching ratios giving the relative populations of $(v=n / v=2$ with $n=1$ and 3$)$ for $\mathrm{NO}(v=2)$ scattering from $\mathrm{Au}(111)$ as function of the incidence translational energy and for different surface temperatures $T_{S}$. Reprinted figure with permission from (55). Copyright 2000 by the American Physical Society. The vibrational relaxation (branching $\operatorname{ratios}(v=1 / v=2)$ ) as well as the excitation (branching ratios $(v=3 / v=2)$ ) increase with incidence translational energy. The vibrational excitation requires thermally excited electron-hole pairs and is thus strongly dependent on $T_{\mathrm{S}}$. The relaxation is rather independent of $T_{S}$. Right: Branching ratios $(v=n /(v=1+v=2+v=3))$ for NO $v_{\mathrm{i}}=3$ scattering from $\mathrm{Au}(111)$. Reprinted with permission from (56). Copyright 2014, AIP Publishing LLC. 
but this has not been tested so far. Instead, e-h pair excitation was verified via electron emission. In this approach, the work function $\phi_{S}$ of the gold surface was lowered from $5.3 \mathrm{eV}$ (clean $\mathrm{Au}(111))$ to $1.6 \mathrm{eV}$ via adsorption of sub-monolayers of Cs. If was found, that molecules prepared in a vibrational state higher than the surface work funtion result in the emission of electrons into the vacuum, see Fig. 2.10A. This could only be explained via a mechanism, in which the vibrational energy is transferred to a single electronic excitation that allows for an electrons to cross the energetic barrier of the surface work function.(57) Katz et al. suggested a reaction mechanism in which a long-range harpooning electron transfer to a molecular ion. The ion is then accelerated towards the surface and an electron is released upon impact of the accelerated ion.(58) The suggestion of the coupling of multiple vibrational quanta to a single electron was further supported by measurements of the kinetic energy of the emitted electrons (Fig. 2.10B).(59) Interestingly, the quantum yield was found to be decreasing with increasing incidence translational energy of surface scattered molecules (Fig. $2.10 \mathrm{C}$ ). This is the opposite trend that has been observed for the efficiency of vibrational excitation and relaxation discussed previously. This inverse velocity dependence of the electron emission has been explained in a special mechanism vibrational autodetachment mechanism, in which electron emission only occurs before a certain critical molecule-surface distance.(60)

\subsection{The Huang experiment}

The vibrational relaxation experiment of $\mathrm{NO}(v=15)$ upon scattering from $\mathrm{Au}(111)$ - which I named the Huang experiment in the introduction of this thesis - provided the foundation this work is based upon. In this section, I will discuss this experiment in more detail.

\subsubsection{Energy transfer in the Huang experiment}

As already described in Fig. 1.1 the scattering of $\mathrm{NO}(v=15)$ with $\mathrm{Au}(111)$ leads to a very efficient vibrational relaxation. Huang et al. (1) further measured angular distributions of surface scattered molecules. These were found to be very narrow, consistent with a direct scattering process (not trapping followed by desorption). This means, that the vibrational energy is lost on a sub-ps timescale (the collision time). 


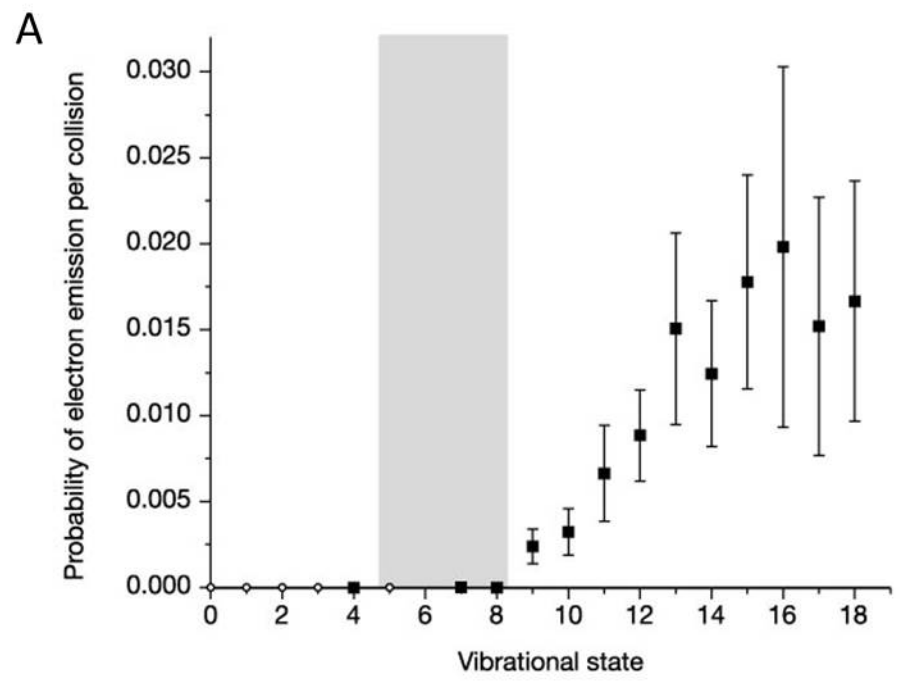

B

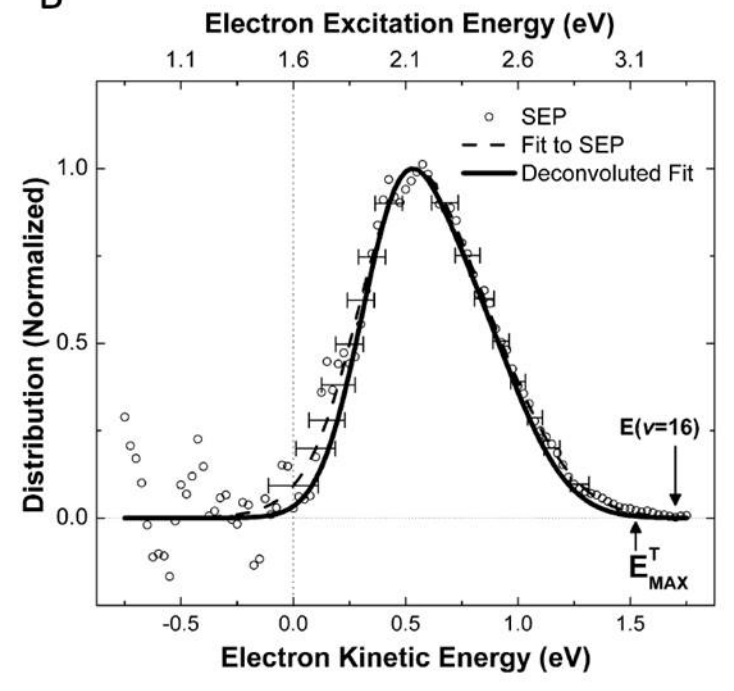

C

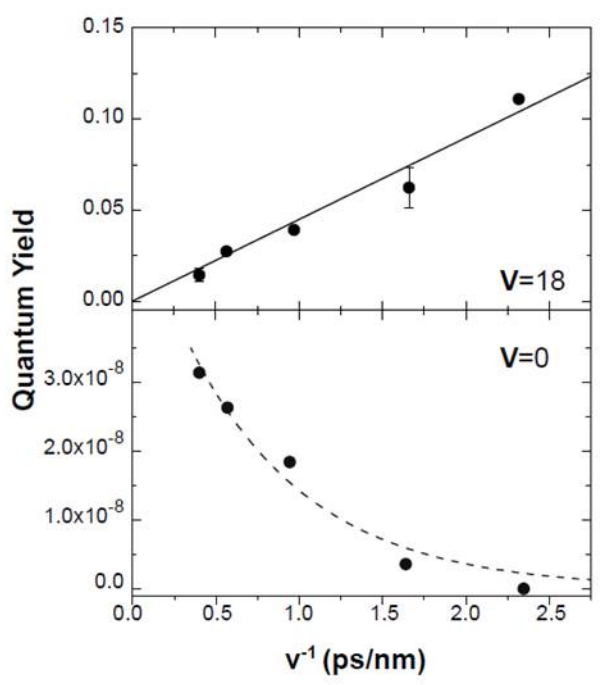

Figure 2.10: Results for the electron emission for NO scattering from a low work-function Cs/Au surface - (A) Probability of eletron emission for NO scattering from a low work-function $\mathrm{Cs} / \mathrm{Au}$ surface as function of the $\mathrm{NO}$ vibrational state. The emission probability increases rapidly when the incident vibrational energy exceeds the surface work function of $1.6 \mathrm{eV}$ ( $\mathrm{NO} v_{\mathrm{i}}=6$ corresponds to a vibrational energy of $1.34 \mathrm{eV}$ and $v_{\mathrm{i}}=7$ corresponds to $1.55 \mathrm{eV}$ ). Reprinted by permission from Macmillan Publishers Ltd: Nature (Ref. (57)), copyright (2004). (B) Kinetic energy distributions of the emitted electrons produced by collisions of $\mathrm{NO}\left(v_{I}=16\right)$. The translational energy is high. The most probable kinetic energy of $0.5 \mathrm{eV}$ corresponds to an energy transfer in which $64 \%$ of the vibrational energy available in the collision is transferred to the electron. This suggests the coupling of several quanta of vibrational energy to a single electronic excitation. Reproduced from Ref. (59) with permission from the PCCP Owner Societies. (C) The quantum yield of the electron emission for $v_{\mathrm{i}}=18$ was found to increase linearly with the inverse velocity of the molecular beam. From (60). Reprinted with permission from AAAS. 


\subsection{The Huang experiment}

How do we know, the loss of vibrational energy is transferred to electron-hole pairs and thus is non-adiabatic?

In order to answer this question, it is important to understand that any gas-surface collision conserves the total energy of a system. The molecule in its electronic ground state has vibrational energy $E_{\mathrm{vib}}$, rotational energy $E_{\text {rot }}$ and translational energy $E_{\text {trans }}$. If the sum of these energies is different prior and after a collision with a surface, it means that energy $\Delta E$ was transferred to the surface.

$$
E_{\mathrm{vib}}^{\mathrm{i}}+E_{\mathrm{rot}}^{\mathrm{i}}+E_{\mathrm{trans}}^{\mathrm{i}}=E_{\mathrm{vib}}^{\mathrm{s}}+E_{\mathrm{rot}}^{\mathrm{s}}+E_{\mathrm{trans}}^{\mathrm{s}}+\Delta E
$$

This energy can be taken up in the form of phonons $E_{\mathrm{ph}}$ or electronic excitations $E_{\mathrm{eh}}$.

$$
\Delta E=E_{\mathrm{ph}}+E_{\mathrm{eh}}
$$

Prior to the collision, the NO molecule in the Huang experiment has energies of $E_{\mathrm{vib}}^{\mathrm{i}}=$ $3.12 \mathrm{eV}(v=15), E_{\mathrm{rot}}^{\mathrm{i}} \approx 0 \mathrm{eV}$ (rotational ground state) and $E_{\mathrm{trans}}^{\mathrm{i}}=0.05 \mathrm{eV}$. After the collision, the most highly populated vibrational state measured was $v^{\mathrm{s}}=7\left(E_{\mathrm{vib}}^{\mathrm{s}}=\right.$ $1.55 \mathrm{eV})$. The rotational excitation was measured and mentioned to be small, but not explicitly given by Huang et al. In this work it will be determined to $E_{\mathrm{rot}}^{\mathrm{s}} \approx 0.03 \mathrm{eV}$ under similar conditions. The translational energy of scattered molecules has not been measured. However, for $v_{\mathrm{i}}=3$ it was recently found (61) that the final translational energy - at least to a first approximation - follows the expectation from a hard sphere collinear collision model (the Baule limit) given by (62)

$$
E_{\text {trans }}^{\mathrm{s}}=E_{\text {trans }}^{\mathrm{i}}\left(\frac{m_{\mathrm{Au}}-m_{\mathrm{NO}}}{m_{\mathrm{Au}}+m \mathrm{NO}}\right)^{2} \approx 0.5 E_{\text {trans }}^{\mathrm{i}}
$$

This gives a value of $E_{\text {trans }}^{\mathrm{s}} \approx 0.03 \mathrm{eV}$. Although there are small deviations in detail, the incidence translational energy is to a first approximation divided into the scattered translation and rotational excitation of surface scattered molecules. This means, that an energy of $\Delta E \approx E_{\mathrm{vib}}^{\mathrm{i}}-E_{\mathrm{vib}}^{\mathrm{s}}$ is transferred to the surface. For $v^{\mathrm{s}}=7$ this corresponds to $\Delta E \approx 1.55 \mathrm{eV}$. For comparison, the energy of a $\mathrm{C}-\mathrm{C}$ single bond (a bond that chemists typically like to form or break) is approximately $3.6 \mathrm{eV}$.

Already the comparison of the vibrational relaxation on $\mathrm{Au}(111)$ to the relaxation on $\mathrm{LiF}$ (almost no relaxation, see Fig. 1.1) suggests that the very efficient energy transfer cannot result from phonon excitation. Indeed, the coupling to phonons would 


\section{Theory and previous results}

be expected to be stronger for $\mathrm{LiF}$ than $\mathrm{Au}$, because $\mathrm{LiF}$ has higher energy phonon modes according to its phonon dispersion curve (highest frequency LiF: $<667 \mathrm{~cm}^{-1}$ (63), Au: $<160 \mathrm{~cm}^{-1}(\underline{64})$. This means, that the frequency mismatch between the phonon modes to the NO stretch $\left(\approx 1904 \mathrm{~cm}^{-1}\right)$ is even larger for $\mathrm{Au}$ then for $\mathrm{LiF}$. Thus, the vibrational energy must be transferred to electronic degrees of freedom. This picture is consistent with the other experiments for the vibrational dynamics of NO scattering from $\mathrm{Au}(111)$ described in section 2.3.3.

\subsubsection{Electron transfer in the Huang experiment}

Following previous theoretical work for the explanation of the vibrational energy transfer on metal surfaces (65), Huang et al. suggested, that the non-adiabatic vibrational relaxation was driven by an electron transfer event between the molecule and the surface.(1) To support this picture, Huang et al. analyzed the electron transfer energetics of the $\mathrm{NO}$ on $\mathrm{Au}(111)$ system, see Fig. 2.11. The left panel of Fig. 2.11
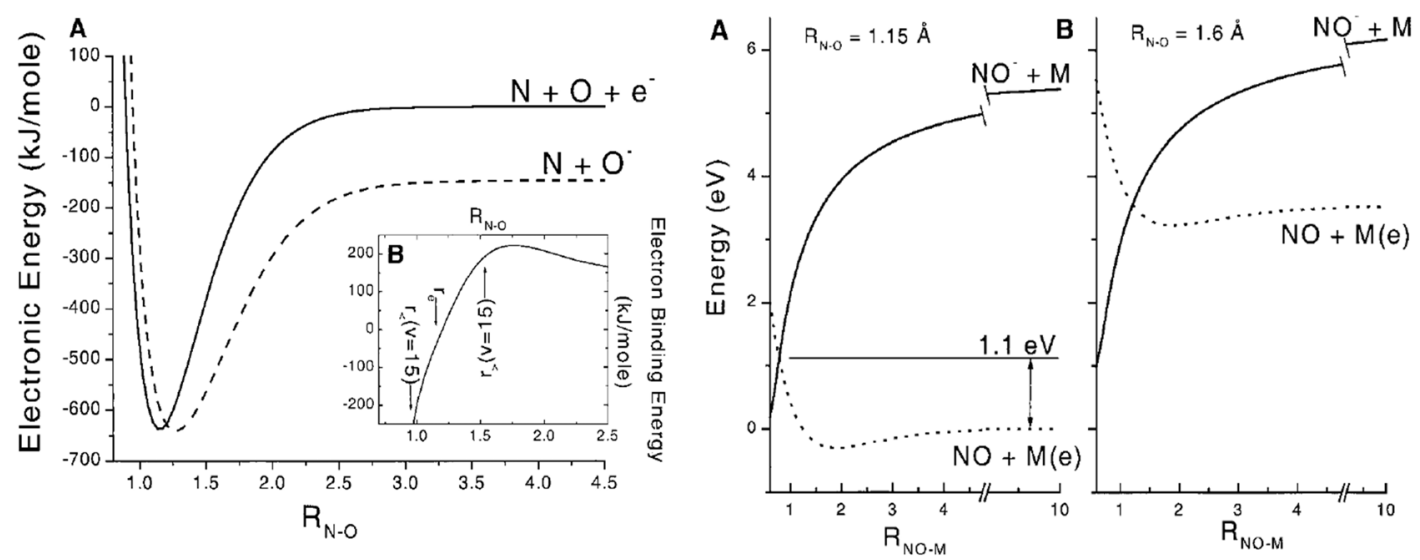

Figure 2.11: Electron transfer energetics for NO scattering from $\mathrm{Au}(111)-$ Left: Ab intio calculations of NO (solid line) and $\mathrm{NO}^{-}$(dashed line) potentials. The inset shows the difference between the potentials or - in other words - the vertical electron binding energy (VEBE). The VEBE is strongly dependent on the molecule-surface distance and changes sign between the inner and outer classical turining points of the vibration (labeled as $r_{<}(v=15)$ and $r_{>}(v=15)$, repsectively). Right: Energetics of the $\mathrm{NO}^{-}+\mathrm{M}^{+}$ and $\mathrm{NO}+\mathrm{M}$ potentials ( $\mathrm{M}$ stands for metal). The $\mathrm{N}-\mathrm{O}$ separation is contrained to the molecules equilibrium bond length $(1.15 \AA)$ in $(\mathrm{A})$ and to the outer classical turning point of the vibration $\left(r_{>}(v=15)=1.6 \AA\right)$ in $(\mathrm{B})$. The barrier for electron transfer vanishes for the extended bond length. Both figures from (1). Reprinted with permission from AAAS. 
shows the energy of $\mathrm{NO}$ and $\mathrm{NO}^{-}$species in the gas-phase. Depending on the $\mathrm{N}-\mathrm{O}$ separation, energy is gained or lost when an electron is attached/detached to the NO molecule.

The energetics of the NO molecule/ion near a $\mathrm{Au}(111)$ surface (metal M) are described in the right panel of Fig. 2.11. The difference between the anionic $\mathrm{NO}^{-}+\mathrm{M}^{+}$potential and the $\mathrm{NO}+\mathrm{M}$ potential in the limit if a large NO-M separation is given by the difference of the surface workfunction $\phi_{\mathrm{S}}=5.3 \mathrm{eV}$ and the vertical electron binding energy (VEBE). When the molecule approaches the surface, the anionic potential is stabilized by the image charge effect (see section 2.2). This brings the neutral and anionic potentials closer in energy. When the NO molecule is at its equilibrium bond lengths $(1.5 \AA)$, there is an energetic barrier towards electron transfer $(1.1 \mathrm{eV}$ within this simple model). However, this barrier vanishes, when the $\mathrm{N}-\mathrm{O}$ bond is stretched. This means, that at a close molecule-surface distance the vibration induces a charge flow between the molecule and the surface. Following the work of Huang et al., I will label this transient, but significant transfer of electronic charge as electron transfer.

\subsubsection{Theoretical calculations for the Huang experiment and their predictions}

The „Huang experiment“ motivated several workgroups from computational chemistry to simulate the scattering experiment with calculations applying methods that go beyond the Born-Oppenheimer approximation.(2, 66, 67, 68, 69) I would like to briefly introduce the two most recent studies(2, 69). Both of them reproduced the experimental results available before I started my $\mathrm{PhD}$ and made some testable predictions for how the vibrational relaxation should change under different experimental conditions, such as the incidence translational energy of the NO molecules.

The study from Shenvi et al. from the group of J. Tully (Yale University) used an algorithm called Independent Electron Surface Hopping (IESH)(70) to account for electronically non-adiabatic transitions between different electronic potential energy surfaces (points for the adiabatic PES of the ground state were calculated with density functional theory).(2, 67) This six-dimensional calculation (frozen surface approximation) assumed that the molecule can exist in only two configurations (neutral and anionic) and treats the motion of the atoms classically. It was predicted, that the amount of vibrational energy transferred to the surface should 


\section{Theory and previous results}

1. be strongly orientation dependent and higher for an N-atom first approach than for an $\mathrm{O}$-atom first approach

2. be higher for molecules with low incidence translational energy than for molecules with high incidence translation (the study compared molecules with $E_{\text {trans }}^{\mathrm{i}}=0.05 \mathrm{eV}$ and $0.8 \mathrm{eV})$.

These trends were explained by the finding that — within the model — the relaxation strongly depends on how close the molecule/ion approaches the surfaces and how much charge is transferred, see Fig. 2.12. According to the calculations, NO molecules oriented with $\mathrm{N}$-atom towards the surface approach the surface much closer and the wavefunctions have stronger anionic contributions, than for molecules oriented with the O-atom towards the surface. The explanation for the prediction, that slow molecules should relax better was that faster molecules do not necessarily penetrate closer to the surface than slower molecules. Instead, the authors suggested, that dynamical steering effects at low translational energy reorient an incident molecule to a more favorable configuration for close approach.

In the second study from Monturet and Saalfrank (Universität Potsdam) nonadiabatic couplings were introduced via electronic friction based on a perturbative treatment of weak vibration-electron couplings.(69) In this purely quantum mechanical, first principles calculation the only degrees of freedom were the molecule surface distance and the internuclear separation. The potential $V(z, r)$ was calculated with an orientation fixed with the $\mathrm{N}$-atom oriented end-on towards the surface (molecular axis perpendicular to the surface), with a position over the surface given by the minimum energy adsorption site. Within this model, the loss of vibrational energy occurs stepwise (as opposed to the direct multi-quantum relaxation within IESH), meaning that the molecule looses one quantum of vibrational energy at a time. The model predicts the most efficient vibrational relaxation at low translational energies (just as in the IESH model) as this gives the molecule more time in the vicinity of the surface. The influence of orientation was not investigated. 


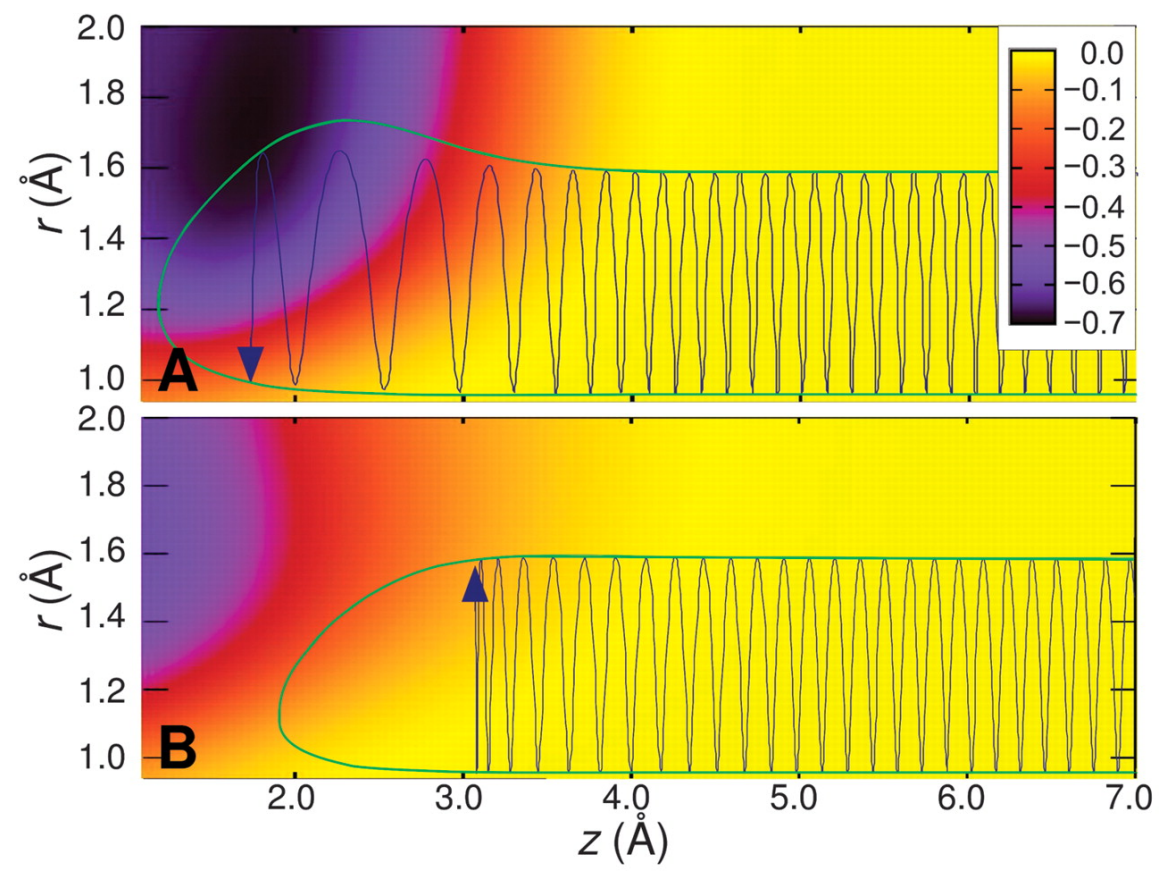

Figure 2.12: Theoretical treatment of the vibrational relaxation of NO on $\mathrm{Au}(111)$ within a theoretical model using the IESH algorithm - The oscillating curves show sample trajectories of vibrating NO molecules (the initial vibrational energy corresponds to a vibrational state of $\left.v_{\mathrm{i}}=15\right)$. The molecular axis is oriented along the surface normal either with the $\mathrm{N}$-atom towards the surface (top panel) or with the $\mathrm{O}$-atom towards the surface. $r$ is the $\mathrm{N}-\mathrm{O}$ internuclear separation and $z$ is the distance of the incoming molecule (center of mass) from the surface. The background colours in the figure indicate the partial negative charge on the NO molecule (meaning the partial anionic character of the wave function). According to the model, molecules exibit stronger vibrational relaxation when oriented with the $\mathrm{N}$-atom towards the surface, as the molecule enters deeper into the region of strong non-adiabatic coupling. The calculation was performed for an incidence translational energy of $0.05 \mathrm{eV}$ at normal incidence above a three-fold hollow site. From (2). Reprinted with permission from AAAS. 

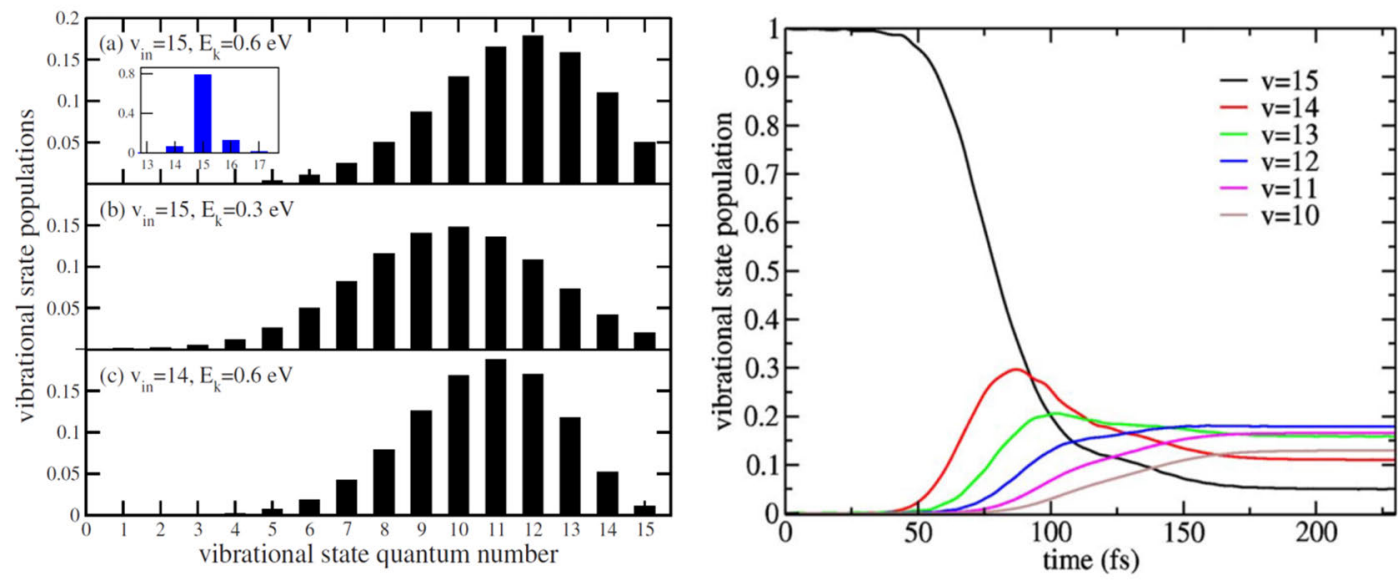

Figure 2.13: Vibrational relaxation of $\mathrm{NO}$ on $\mathrm{Au}(111)$ within friction theory (left) Calculated vibrational state distributions for different incidence vibrational states and translational energies. The model predicts less vibrational relaxation at higher incidence translational energy, compare panels (a) and (b). (right) Development of the vibrational state distribution as function of time. At $t=0$, only the 15 th vibrational state is occupied. Figures reprinted with permission from (69). Copyright 2010 by the American Physical Society.

\subsubsection{The challenge of testing the theoretical predictions}

The theoretical calculations I just reviewed made predictions regarding the dependence of the vibrational relaxation effect on the incidence translational energy and one of them also suggested a strong dependence on the molecular orientation. Why did Huang et al. not vary these parameters earlier?

One of the main problems of the experiment in the way it was done by Huang et al. in 2000 was that the experiment took an incredible long time. Although Huang et al. provided data for the vibrational states between $v=5$ and $v=15$ only, determining the population of these vibrational states with resonance enhanced multi photon ionization (REMPI) spectroscopy required spectra in a wavelength brange between $270 \mathrm{~nm}$ and $350 \mathrm{~nm}$ (frequency doubled), which corresponds to a range of $540 \mathrm{~nm}$ to $700 \mathrm{~nm}$ in the fundamental. This was done with a dye laser. Covering this wavelength range with reasonable laser power required the use of approximately 10 different laser dyes and measuring the one vibrational state distribution they published took approximately one year. For the work presented in this thesis, a more modern solid state laser system was purchased which can cover the entire wavelength range without changing laser 
dyes (section 3.3.4). Another problem was that the technique used for the vibrational state preparation - Stimulated emission pumping (SEP) - produced a background in undesired vibrational states in the optical pumping. The way we solved this problem is explained in chapter 5. Finally, investigating the influence of the molecular orientation in molecule-surface scattering experiment is experimentally challenging and also required some method development. I will address this topic in chapter 4.

\subsection{Orientation in surface dynamics}

The importance of molecular orientation has been extensively studied previously for several systems within the field of surface dynamics and has been reviewed previously (71). These experiments use an orientation method based on hexapole state-selection, see section 4.2. The molecular orientation has been found to influence sticking probabilities. In particular, the sticking of $\mathrm{NO}$ on $\mathrm{Ni}(100)$ and $\mathrm{Pt}(100)$ as well as the dissociative adsorption of $\mathrm{NO}$ on $\mathrm{Si}(111)(7 \times 7)$ were found to occur at higher probability for molecules oriented with the $\mathrm{N}$-atom towards the surface than for molecules oriented with the O-atom towards the surface.(72, 73, 74) A weak orientation dependence has also been found for the sticking probability of $\mathrm{CH}_{3} \mathrm{Cl}$ on $\mathrm{Si}(100)$, which was observed to be higher for a $\mathrm{Cl}$-end collision in comparison to a $\mathrm{CH}_{3}$-end collision.(75) The influence of the orientation has also been studied for the formation of $\mathrm{CO}_{2}$ when an oriented $\mathrm{NO}$ molecular beam impinges a $\mathrm{CO}$ covered $\mathrm{Pt}(111)$ surface. In this case, the orientation effect is strongly coverage dependent. At high $\mathrm{CO}$ coverage the $\mathrm{CO}_{2}$ formation is enhanced by $\mathrm{N}$-atom first orientation and is believed to proceed dominantly in a direct scattering process via an Eley-Rideal mechanism.(76, 177)

Brandt et al. measured the electron emission in collisions of vibrationally excited $\mathrm{N}_{2} \mathrm{O}$ (one quantum excitation in the $\nu_{2}$ bending mode) with 1 monolayer of $\mathrm{Cs}$ on $\mathrm{Pt}(111)$, see Fig. 2.14.(78) They found, that the electron emission is strongly enhanced for $\mathrm{O}--$ end collisions and only occurs for the vibrationally excited molecule. Brandt et al. suggested a mechanism for the electron emission that involves a harpooning electron jump from the surface to the $\mathrm{O}$ end of the $\mathrm{N}_{2} \mathrm{O}$ molecule, followed by dissociation of the molecule and $\mathrm{O}^{-}$hitting the surface. In their mechanism, the hole on the $\mathrm{O}^{-} 2 \mathrm{p}^{5}$ ion dives to energy levels deeper than the work function and an electron is emitted in an Auger type mechanism with the formation of a closed-shell $\mathrm{O}^{2-}$ like ground-state. 

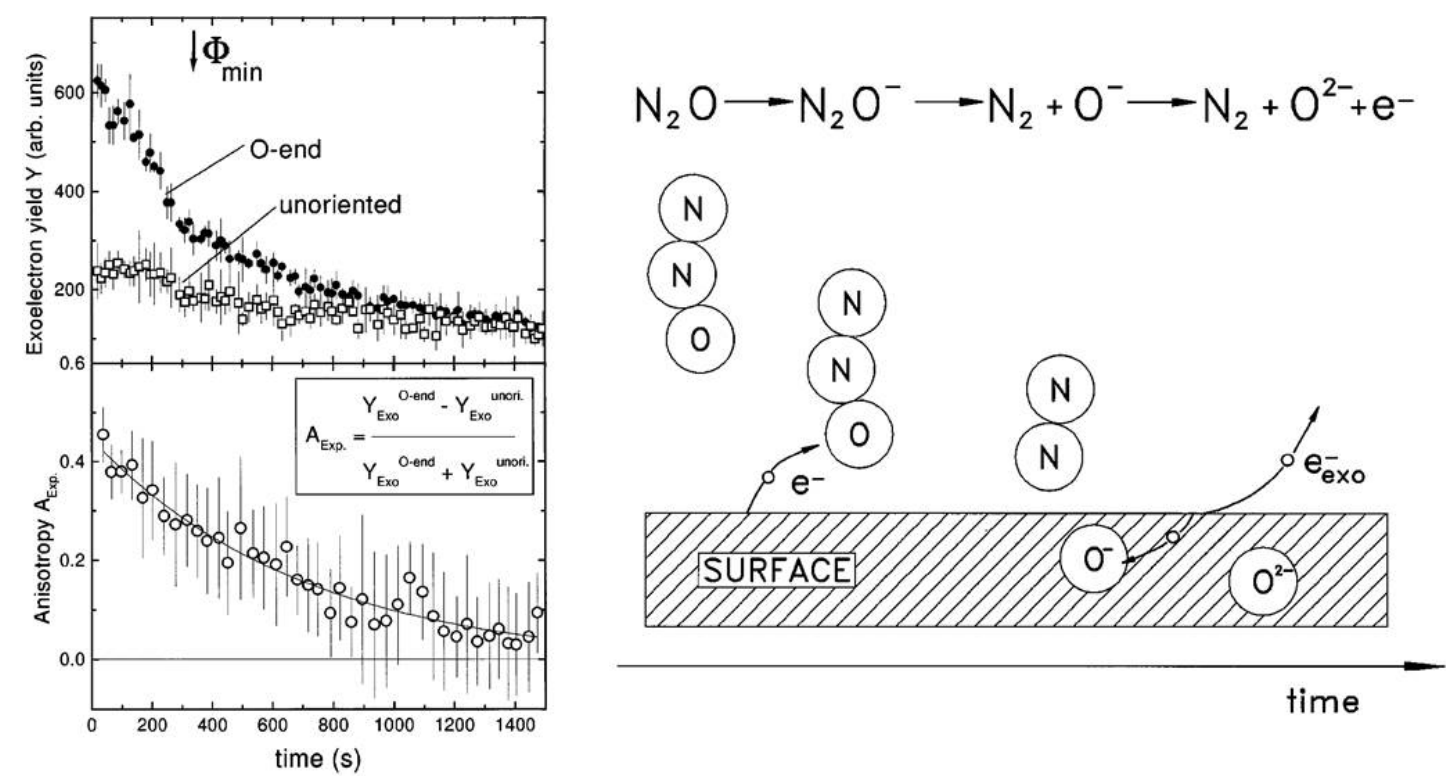

Figure 2.14: Orientation dependent electron emission when a Cs/Pt(111) surface is oxidized by $\mathrm{NO}_{2}$ - Left: The top panel shows the electron yield when $\mathrm{NO}_{2}$ $\left(\nu_{2}=1\right)$ is scattered from a $\mathrm{Cs} / \mathrm{Pt}(111)$ surface either pointing with the $\mathrm{O}$-end towards the surface or isotropically oriented. The bottom panel shows the corresponding anisotropy as defined in the figure legend. Right: Proposed mechanism for the electron emission. The $\mathrm{N}_{2} \mathrm{O}$ molecule is resonantly ionized and the attraction between the negative charge on the $\mathrm{N}_{2} \mathrm{O}^{-}$anion and the hole in the surface accelerate the anion (harpooning mechanism). The anion dissociates $\left(\mathrm{N}_{2}\right.$ leaves) and $\mathrm{O}^{-}$hits the surface. The $\mathrm{O}^{-}$anion is reduced to $\mathrm{O}^{2-}$ and an electron is emitted in an Auger process. Reprinted figures with permission from (78). Copyright 1998 by the American Physical Society. 
This argumentation is however still controversially discussed.(79)

In any case, it is well known from other fields of chemistry, that energy transfer as well as electron transfer are strongly orientation dependent. For example, Förster energy transfer favors specific relative directions of each molecule's transition dipole (80) and electron transfer in the gas-phase is explained in terms of the relative orientation of donor and acceptor orbitals(81).

\subsection{Rotational excitation in gas-surface collisions}

The incidence molecular orientation was also found to have a strong impact on the rotational excitation in gas-surface collsions. This topic created a lot of interest in the 1980s and early 1990s and was referred to as rotational rainbow scattering. I would like to explain this on the example of the scattering $\mathrm{NO}(v=0)$ from $\operatorname{Ag}(111)$. This and other examples can be found in a review article from A. W. Kleyn.(82)

Figure 2.15 shows early results from Kleyn et al. for the rotational state distributions when NO scatters from $\mathrm{Ag}(111)$. (83) The rotational excitation was found not to result from equilibration with the surface temperature and actually non-thermal; the excitation increases with the incidence translational energy. This was explained by a direct scattering process in which incidence translational energy in converted to rotational energy of surface scattered molecules. An interesting feature of the rotational state distributions was that they appeared to have two components: one component at low rotational energy (originally labelled as thermal) and one component with a maximum at high rotational energy which was called rotational rainbow. Already in this early work it was suspected, that the rotational structure could only be explained by an interaction potential between the NO molecule and the surface that strongly depends on the orientation angle.(83)

The orientation dependence of the rotational excitation was later confirmed in experiments using oriented NO molecular beams. Fig. 2.16 shows results for the scattering of NO from $\mathrm{Ag}(111)$ from Geuzebroek et al. for the highest incidence translational energy of $0.34 \mathrm{eV}$ where orientation could be achieved with the hexapole orientation technique. The data proves, that the high $J$ component of the rotational state distributions results predominantly from collisions in which the $\mathrm{O}$-atom in pointing towards the surface, whereas $\mathrm{N}$-atom first collisions produce population in lower rotational 


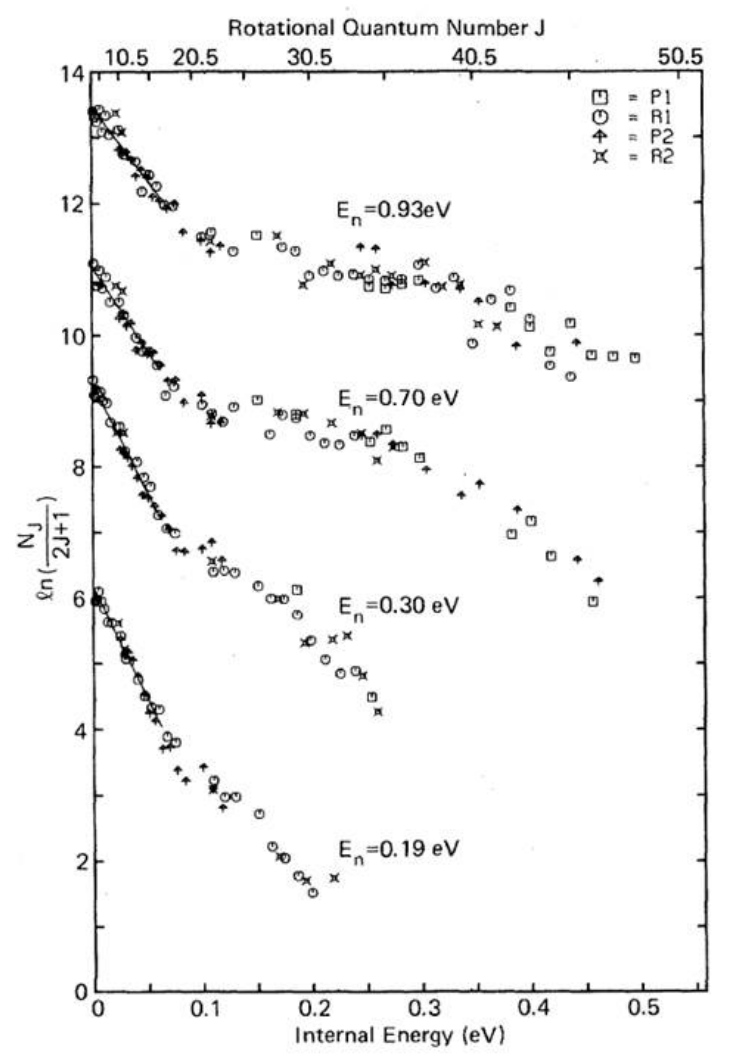

Figure 2.15: Boltzmann plot of the rotational state distributions of NO scattering from $\mathrm{Ag}(\mathbf{1 1 1})$ - Rotational-state distributions for $\mathrm{NO}$ scattered from $\mathrm{Ag}(111)$ as a function of internal energy (bottom scale) or rotational quantum number $J$ (top scale). The $J$-scale shown applies to the $\Omega=1 / 2$ spin-orbit state. From top to bottom: $E_{\text {trans }}^{i}$ $=1.0 \mathrm{eV}, \theta_{\mathrm{i}}=15^{\circ} ; E_{\text {trans }}^{\mathrm{i}}=0.75 \mathrm{eV}, \theta_{\mathrm{i}}=15^{\circ} ; E_{\text {trans }}^{\mathrm{i}}=0.32 \mathrm{eV}, \theta_{\mathrm{i}}=15^{\circ} ; E_{\text {trans }}^{\mathrm{i}}=$ $0.32 \mathrm{eV}, \theta_{\mathrm{i}}=40^{\circ}$. The energies given as $E_{n}$-labels are the components normal to the surface. Molecules were detected at specular angle. Reprinted (abstract/excerpt/figure) with permission from (83). Copyright 1981 by the American Physical Society. 
states.(84) The rotational rainbow scattering was also discussed in several theoretical

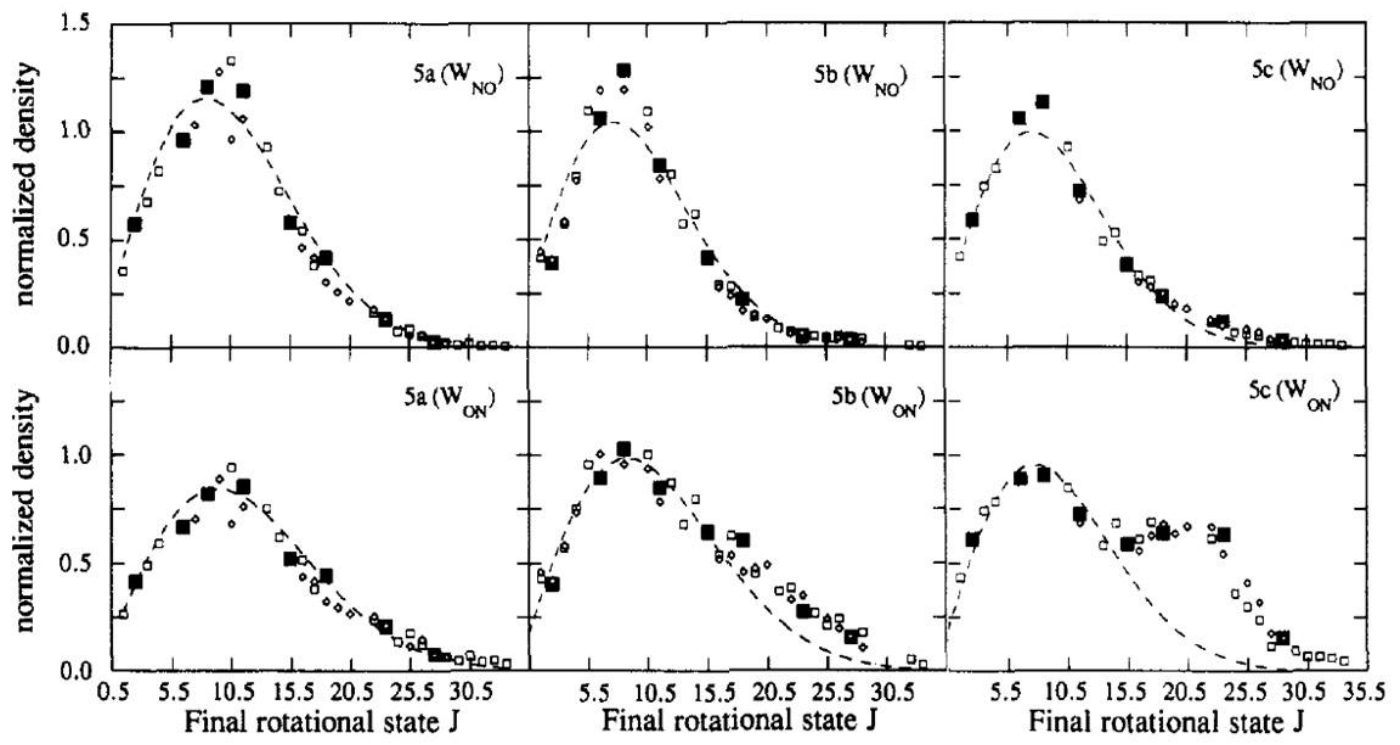

Figure 2.16: Rotational state distributions for oriented NO scattering from $\operatorname{Ag}(\mathbf{1 1 1})$ - The top panels show the scattering of a molecular beam with enhanced $\mathrm{N}-$ atom first orientation and the bottom panels for enhanced $\mathrm{O}$-atom first approach. The molecules impinge the surface at an incidence scattering angle of $-45^{\circ}$ at a translational energy of $0.34 \mathrm{eV}$. The data is given for scattering angles of $35^{\circ}$ (towards the surface normal, a), $53^{\circ}$ (b), and $70^{\circ}$ (towards the surface, c). The strongest high $J$ rotational rainbow is observed for $\mathrm{O}$-atom first collsions for molecules scattered towards the surface (bottom right panel). Reprinted with permission from (84). Copyright (1991) American Chemical Society.

studies (see (82, 85, 86, 87) and references therein). All of these studies reduced the problem to the elastic scattering of a rigid rotator from a flat surface (no surface corrugation and no surface motion) and two dimensional (orientation angle $\theta$ and molecule surface distance $z$ ) model potentials were developed. These were refined by comparing the results of classical or quantum mechanical trajectory calculations to the experimental rotational state distributions.

One of the most recent model potentials was suggested by Tenner et al.(87, 88) in 1990. This potential refines a model potential originally developed by Voges and Schninke (VS potential)(85) with the addition of a second term for a deeper attractive well depth and is thus called VSW potential. This potential reproduces experimental results for the orientation dependent scattering of NO from $\mathrm{Ag}(111)$ reasonably well. Fig. 2.17 shows 

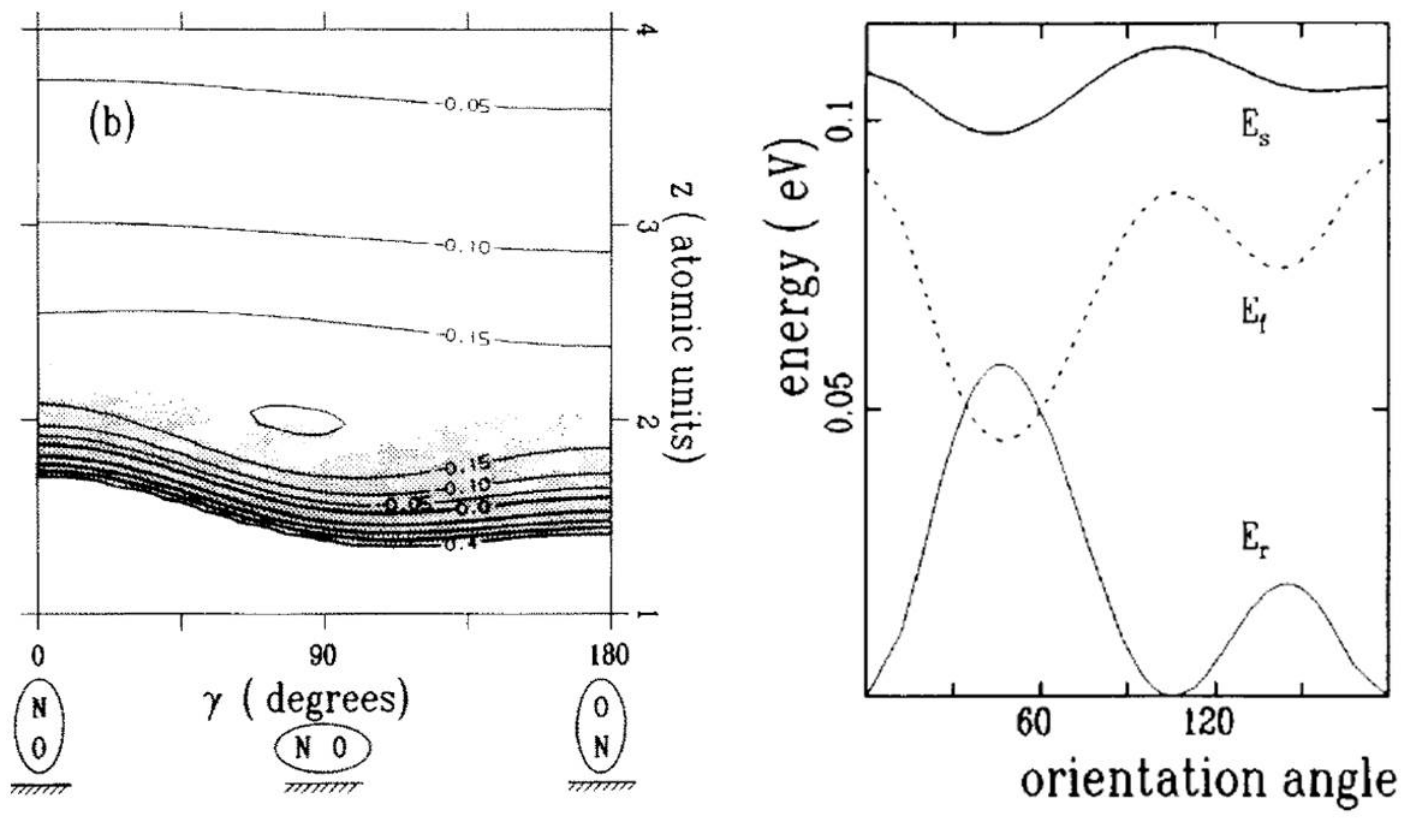

Figure 2.17: Theoretical explanation of the orientation dependent rotational excitation - (left): Contour representation of a model potential energy surface for the $\mathrm{NO} / \mathrm{Ag}(111)$ interaction. The potential has an attractive well of $0.2 \mathrm{eV}$ and is strongly asymmetric with respect to the orientation angle (here labelled as $\gamma$ and called $\theta$ elsewhere in thesis). (right) Classical molecular dynamics simulations on the PES show that the scattered rotational energy (here $E_{\text {rot }}$ ) of the initially non-rotating molecule is also strongly orientation dependent. The calculation was done on an initially stationary surface cube of mass 240 . The scattered energy $E_{\mathrm{s}}$ is also slightly orientation dependent, but always close to the Baule limit of approximately $0.1 \mathrm{eV}$. The remaining energy $\left(E_{\mathrm{s}}-E_{\mathrm{r}}\right)$ is in the final translation $E_{\mathrm{f}}$. The orientation angle is defined as in the left panel. Reprinted from Publication (88) Classical trajectory study of the interaction of oriented NO and $\mathrm{Ag}(111)$, 242/1-3, Tenner et al., 376 - 385, Copyright (1991), with permission from Elsevier. 
a contour plot of the VSW potential as function of the orientation angle and the energy transfer predicted by the model. The potential has an attractive well of $0.2 \mathrm{eV}$ and is strongly asymmetric (more repulsion for an $\mathrm{O}$-first than for an $\mathrm{N}$-first orientation). According to molecular dynamics simulations on that potential, the highest rotational energy is obtained for an orientation angle of approximately $50^{\circ}$ on a scale where $0^{\circ}$ corresponds to an $\mathrm{O}$-atom first end-on collision and $180^{\circ}$ to an $\mathrm{N}$-atom first end-on collision. End-on collisions never lead to rotational excitation for any $V(z, \theta)$ potential, as no torque is exerted to the molcule.

An open question is how the rotational state distributions evolve, in case molecular collisions lead to a change of the vibrational state. Rettner et al.(89) measured very similar rotational state distributions in $v=0$ and $v=1$ in vibrational excitation experiments for NO $v=0$ scattering from $\mathrm{Ag}(111)$, which lead to the conclusion that "rotational and vibrational excitation mechanisms are decoupled and that rotational rainbows are formed in an identical way for both $v=0$ and $v=1$." (82) As opposed to this, Wodtke et al. found that NO $(v=2)$ scattering from $\mathrm{Au}(111)$ lead to less rotational excitation in the vibrationally inelastic channels $(v=2 \rightarrow 1)$ and $(v=2 \rightarrow 3)$ compared to the vibrationally elastic channel $(v=2 \rightarrow 2)$. This was referred to as Rotational cooling associated with vibrational relaxation and was attributed to either an effect of the molecular orientation or the surface corrugation.(90) 
2. Theory and previous results 


\section{3 \\ Experimental}

\subsection{Vacuum chamber with molecular beam}

Figure 3.1 shows a schematic of the surface scattering apparatus used in my Thesis. A pulsed molecular beam of rotationally cold (rotational temperature $\approx 5 \mathrm{~K}$ ) nitric oxide (NO) is generated in a supersonic jet expansion into vacuum through a piezoelectric valve ( $1 \mathrm{~mm}$ diameter nozzle, $10 \mathrm{~Hz}$ repetition rate, 3 atm stagnation pressure, approximate opening time $170 \mu \mathrm{s})$.

The velocity of the molecular beam is controlled by seeding NO in different carrier gases. The beam velocities $v_{f}$ were determined experimentally, but can be approximately estimated with the equation (91)

$$
v_{f}=\sqrt{\frac{2}{\bar{m}} \overline{c_{p}} T_{0}}
$$

where $\bar{m}$ is the average mass of the molecules expended, $\overline{c_{p}}$ is their average heat capacity at constant pressure and $T_{0}$ is the nozzle temperature, which was room temperature in this work. $3 \mathrm{~cm}$ downstream from the nozzle, the beam passes a $2 \mathrm{~mm}$ electro-formed skimmer (Ni Model 2, Beam dynamics Inc.) and enters the differential chamber. This chamber is used for preparing the NO molecules in excited vibrational states. This is done via vibrational overtone pumping to $v=3$ with pulsed infrared laser radiation (the laser system is described in section 3.3.1) or with several UV-Vis laser pulses with the Pump-Dump-Sweep technique (see section 5). In order to control the optical preparation, the chamber is equipped with a photo-multiplier tube detector (PMT, Hamamatsu, R7154). 


\section{Experimental}

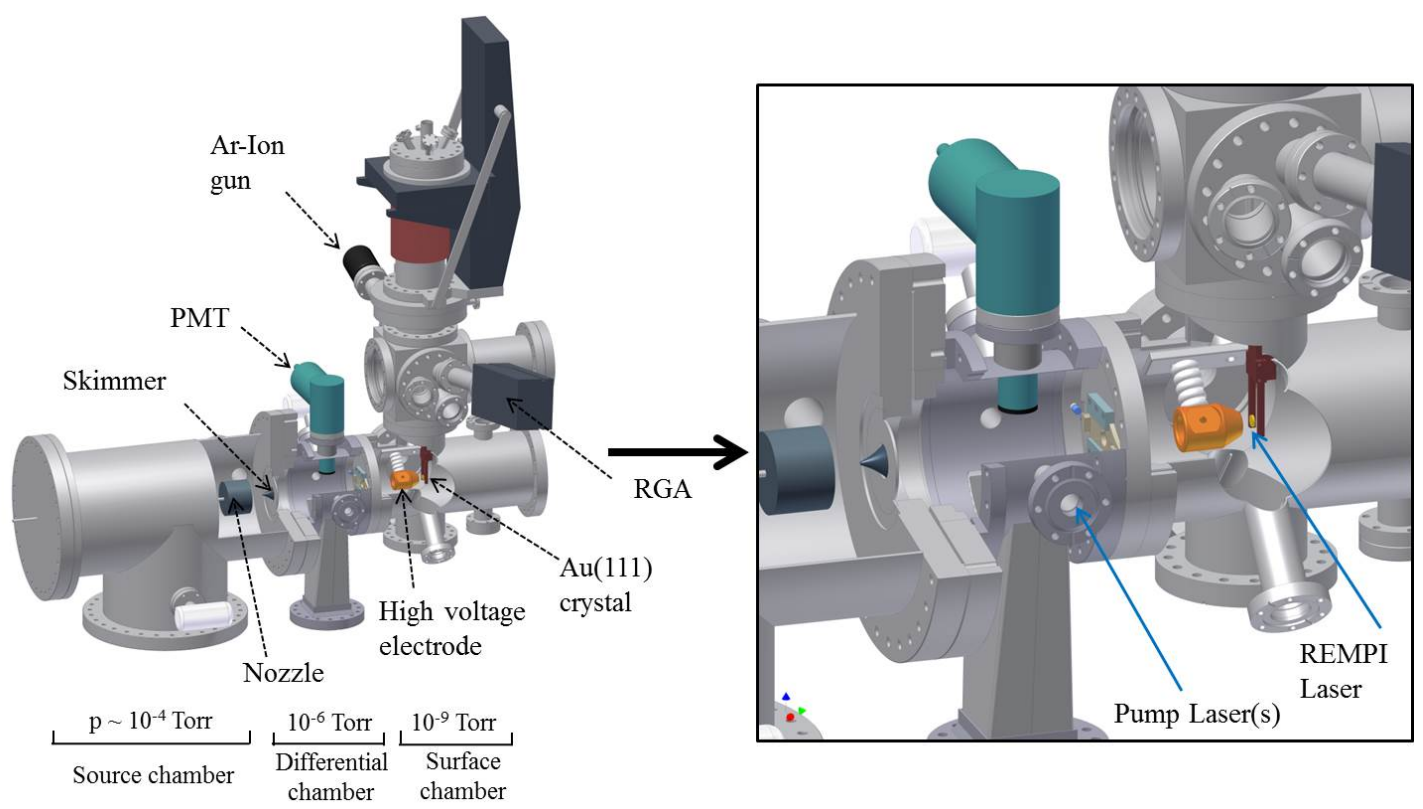

Figure 3.1: Experimental setup - The molecular beam originates at the nozzle on the left side of the figure. Molecules are optically pumped to excited vibrational states in the differential chamber next to the photomultiplier tube (PMT). A high voltage electrode in the surface chamber allows for the orientation of NO molecules prior to their collision with the $\mathrm{Au}(111)$ surface. Ar-Ion gun, Auger spectrometer and residual gas analyser (RGA) are attached to the machine to ensure the cleanliness of the $\mathrm{Au}(111)$ surface. The pressures in the chambers when the molecular beam is running are given as labels. The positions, where pump laser(s) for the vibrational excitation of the NO molecules and the UV REMPI laser for probing scattered state distribtuions cross the molecular beam are shown as blue arrows in the enlargement of the surface chamber (right panel). Defining the nozzle to be at position $x=0$ (with $x$ being along the axis of the molecular beam), the skimmer is at $x=3 \mathrm{~cm}$, the pump lasers cross at $x=12 \mathrm{~cm}$ and the surface at $x=32 \mathrm{~cm}$. 
The molecules then pass through a $2 \mathrm{~mm}$ aperture and enter the surface chamber, where they scatter from a clean $\mathrm{Au}(111)$ single-crystal surface at normal incidence. The number density at the maximum of the molecular beam pulse close to the surface can be calculated from the pressure rise induced by the molecular beam and the pumping speed in the UHV chamber to $10^{11}$ molecules $/ \mathrm{cm}^{3}$.

The setup also allows for orienting the NO molecule in the laboratory frame prior to the collision (see section 4). This requires a high voltage electrode, which is mounted in front of the grounded gold crystal. Voltages between $+15 \mathrm{kV}$ and $-15 \mathrm{kV}$ can be applied to electrode, generating an electric field of up to $\pm 33 \mathrm{kV} / \mathrm{cm}(15 \mathrm{kV}$ at $4.5 \mathrm{~mm}$ electrode-surface distance). Vibrationally elastically and inelastically scattered molecules are eventually detected by means of resonance enhanced multi photon (REMPI) spectroscopy. Ions created by the laser pulses are accelerated onto a micro-channel plate detector (Tectra MCP 050 in chevron assembly). Approximately $1 \mu$ s prior to the REMPI detection, the electric field is pulsed to ground with a high-voltage switch (Behlke, HTS 300). The setup uses the principle of differential pumping to maintain a high vacuum in the surface chamber $\left(\mathrm{p} \approx 1.0 \cdot 10^{-9}\right.$ Torr with running molecular beam and $\approx 1.0 \cdot 10^{-10}$ Torr without molecular beam). This is achieved with four different turbo-molecular pumps which are backed up by rotary-vane pumps. The source and differential chambers are pumped by a 400 l/s (Osaka Vacuum Ltd., TS443) and a 360 l/s (Leybold, Turbovac 360CSV) turbo-molecular pump, respectively. The UHV chamber uses a $420 \mathrm{l} / \mathrm{s}$ (Osaka, TMP062) turbo-molecular pump, which is backed by a 170 l/s turbo-molecular pump (Pfeiffer, TPU 170).

\subsection{Surface preparation and verification of cleanliness}

Every day before scattering experiments were performed, the $\mathrm{Au}(111)$ crystal was cleaned by sputtering for 15 minutes with an Ar-ion gun (LK Technologies, model NGI 3000-SE, beam voltage $3.0 \mathrm{kV}$, emission current $20 \mathrm{~mA}$ ). An ion current of $10 \mathrm{~mA}$ is reached at an Argon pressure of $1.5 \cdot 10^{-6}$ Torr. The surface was then annealed for $20 \mathrm{~min}$ at a temperature of $970 \mathrm{~K}$ via resistive heating with tungsten wires. Surface cleanliness was regularly tested using Auger electron spectroscopy with an Auger spectrometer (Staib Instruments, ESA 100). After any exposure of the $\mathrm{Au}(111)$ surface to atmosphere (e.g. after opening the machine for modifications on the setup), sputtering 


\section{Experimental}

of the Au surface was essential. Fig. 3.2 shows an Auger spectrum of a Au crystal after exposure to atmosphere which was only cleaned by annealing (red solid line). The spectrum reveals surface coverage with carbon and calcium. Sputtering of the Au crystal and subsequent annealing completely removed these impurities below the Auger detection limit (red solid line). I found that a $\mathrm{Au}(111)$ only cleaned by annealing

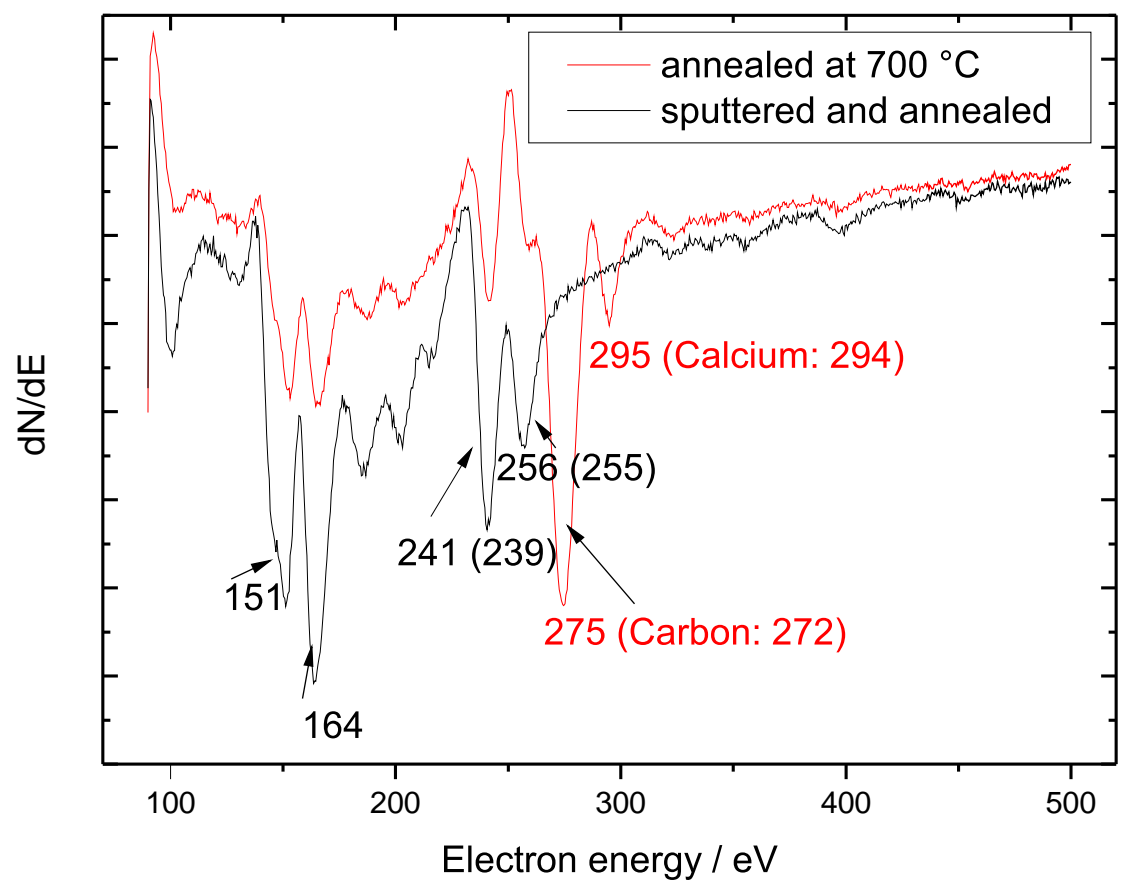

Figure 3.2: Demonstration of the importance of Ar sputtering in the surface cleaning procedure of $\mathbf{A u ( 1 1 1 )}$ - The red solid line shows an Auger electron spectrum of a $\mathrm{Au}(111)$ surface after exposure to air, and subsequent annealing for $15 \mathrm{~min}$ at $700{ }^{\circ} \mathrm{C}$ under UHV conditions. It shows coverage from carbon and calcium. These impurities can be removed by sputtering with an Ar-ion gun and subsequent annealing under the same conditions as previously (black solid line). Red labels indicate the electron energy of Auger peaks assigned as belonging to impurities (carbon and calcium), while black labels belong to peaks from $\mathrm{Au}$. The values in brackets are literature values(92) for the electron energy of the assigned peaks.

behaves almost like an insulator regarding the $\mathrm{NO}$ vibrational relaxation experiments (almost no vibrational relaxation of $\mathrm{NO}$ molecules prepared in $v_{\mathrm{i}}=11$ at an incidence translational energy of $0.48 \mathrm{eV}$ ). This underlines the importance of Ar sputtering in the cleaning procedure. $\mathrm{A} \mathrm{Au}(111)$ surface cleaned with the sputtering and anneal- 
ing procedure was observed to remain clean according to Auger electron spectroscopy and vibrational relaxation probability for weeks. The daily sputtering procedure was nonetheless maintained.

In the current setup it is not possible to characterize the surface structure, e.g. by low-energy electron diffraction (LEED). However, it is well known that $\mathrm{Au}(111)$ surfaces form a $22 \times \sqrt{3}$ "herringbone" reconstructed surface (see Ref. (93) and references therein). While the surface is mostly characterized by an face centered cubic (fcc) toplayer alignment, there are smaller fractions with hexagonal-close-packed (hcp) surface sites. These regions are connected via straight ridges visible with a scanning tunnelling microscope. Existing computational work on the $\mathrm{NO} / \mathrm{Au}(111)$ (see Section 2.4.3) does not take this reconstruction into account.

\subsection{Laser systems}

The vibrational relaxation experiments described in this work were performed using several laser systems for the optical preparation of NO molecules in high vibrational states and the detection of ro-vibrational state distributions after the collision with the Au surface. In particular, molecules were excited to the vibrational state $v_{\mathrm{i}}=3$ with a Fourier-transform limited infrared source (subsection 3.3.1). The preparation of high vibrational states $\left(v_{\mathrm{i}}=11\right.$ and $\left.v_{\mathrm{i}}=16\right)$ was achieved with two home-built, injection seeded optical parametric oscillators (OPOs) (subsection 3.3.1). The quantum state purity in the excited high vibrational states was improved with the output of a commercial dye laser (subsection 3.3.1) in the sweep step of the pump-dump-sweep approach. Finally, the scattered ro-vibrational state distributions were probed with a commercial solid-state OPO laser system (Continuum, Sunlite Ex OPO with FX-1 UV frequency extension) that is widely tunable in frequency. These laser systems are briefly described in this section and references for further reading are provided. All laser systems have in common, that they are pumped by the second or third harmonic of a Nd:YAG laser at a repetition rate of $10 \mathrm{~Hz}$.

\subsubsection{Fourier-transform limited IR source}

For vibrational overtone pumping of NO molecules from the vibrational ground state to $v=3$, I used a high power ns infrared laser system with nearly Fourier-transform 


\section{Experimental}

limited bandwidth. This complex laser system consists of

1. a continuous wave (cw) Nd:YLF laser (Coherent, Verdi-10)

2. a cw ring dye laser (Sirah, Matisse DR)

3. a five stage pulsed amplifier (Sirah, PulsAmp 5X), which is pumped by the second harmonic of an injection-seeded, pulsed Nd:YAG laser (Spectra Physics, QuantaRay Pro-230)

4. a combined difference frequency mixing and parametric amplification unit (Sirah, DFM-2400-T and Sirah, DFA-2400-T)

The laser system actually belongs to a different experimental setup in the same laboratory (the Beamer 1 machine) and has been extensively described in the $\mathrm{PhD}$ thesis from Kai Golibrzuch(10). For the purpose of understanding my Thesis, it is sufficient to know that it is capable of producing intense infrared laser pulses $(\approx 20 \mathrm{~mJ}$ at $1.8 \mu \mathrm{m}$ with $0.006 \mathrm{~cm}^{-1}$ linewidth). This resolution and power is sufficient to populate either the (e) or the (f) parity state in $v=3$ by saturating either of the transitions NO $\mathrm{X}^{2} \Pi_{1 / 2}(v=0, J=0.5, e) \rightarrow \mathrm{X}^{2} \Pi_{1 / 2}(v=3, J=0.5, f)$ (the $\mathrm{Q}_{11}(0.5)_{e}$ line) or NO X $\Pi^{2} \Pi_{1 / 2}(v=0, J=0.5, f) \rightarrow \mathrm{X}^{2} \Pi_{1 / 2}(v=3, J=0.5, e)$ (the $\mathrm{Q}_{11}(0.5)_{f}$ line), see Fig. 6.1 for an energy diagram and Fig. 6.2 for examples of scans of the infrared laser system.

\subsubsection{Home-built narrow-bandwidth optical parametric oscillators}

For the optical pumping of high vibrational states, we used two very similar homebuilt optical parametric oscillators (OPO) laser systems generating tunable, narrowbandwidth $\left(<0.01 \mathrm{~cm}^{-1}\right.$ at $\left.206 \mathrm{~nm}\right)$, nanosecond, laser pulses with high output power. Following the work of Velarde et al.(94) and Mahnke et al. (95), I built one of these OPOs from scratch for my masters thesis(96). The optical design of the OPOs is shown in Fig. 3.3. Two KTP crystals (Altechna Co. Ltd., $\theta=70^{\circ}$ ) mounted in a planar ring cavity (mirrors M1 to M4) are used for the parametric amplifcation. To achieve single-mode operation, the OPOs are seeded with external cavity diode lasers (ECDL, Toptica Photonics, DL Pro 100, 875-940 nm). The concept used for frequency locking is a derivation of the "intensity dip" method introduced by He and Orr(97), 


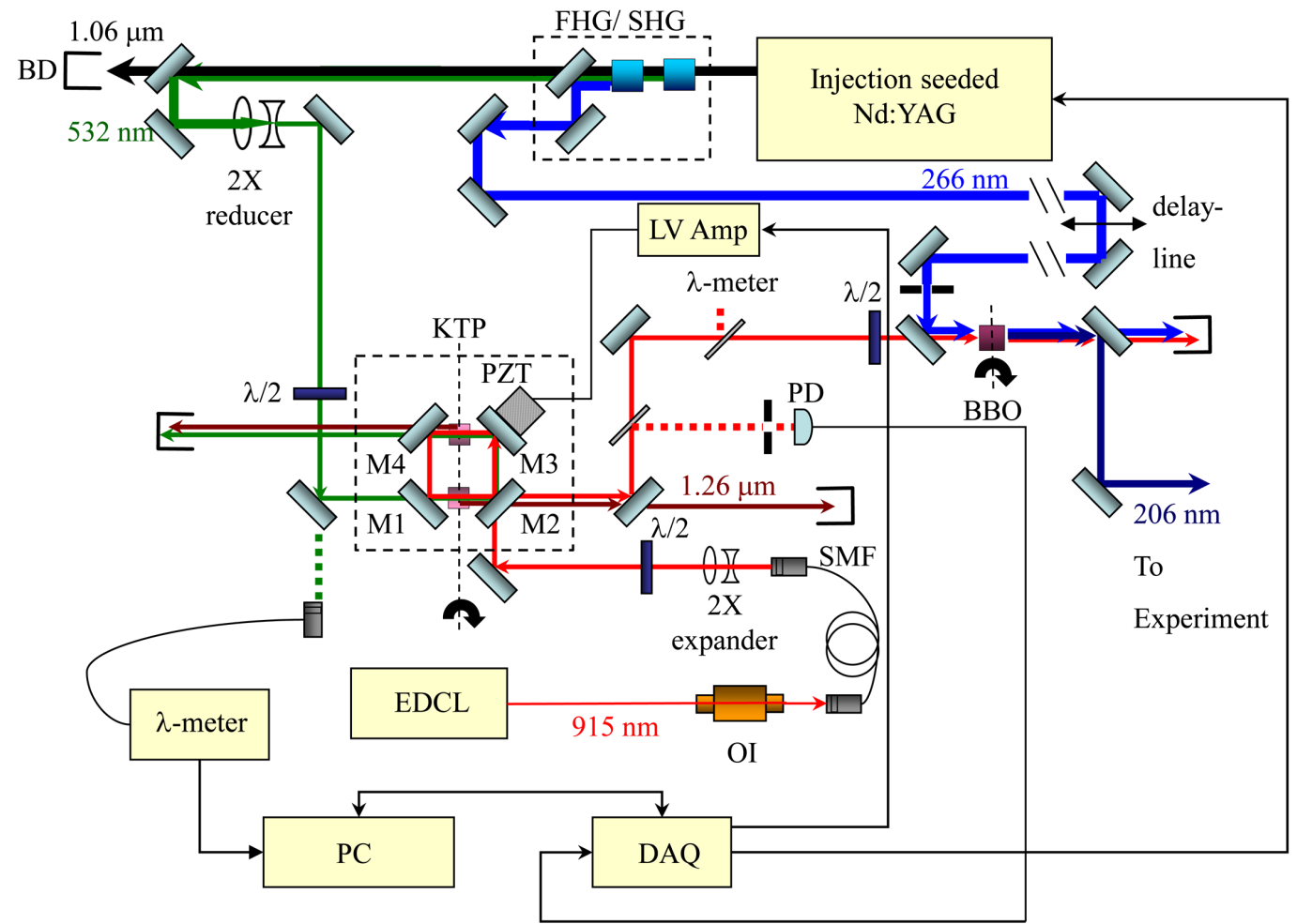

Figure 3.3: Optical layout of the injection seeded OPO - EDCL $=$ external cavity diode laser, $\mathrm{DAQ}=$ data acquisition card, $\mathrm{PZT}=$ piezoelectric transducer, $\mathrm{BD}=$ beam dump, $\mathrm{OI}=$ optical isolator, $\mathrm{PD}=$ photo diode, $\mathrm{SMF}=$ single-mode fiber, $\mathrm{LV} \mathrm{Amp}=$ low voltage amplifer. Figure from my maters thesis (96). 


\section{Experimental}

which is also referred to as ramp, hold and fire technique. The OPOs produce Fourier transform limited laser pulses in the infrared (OPO signal), tunable in the range of 875$940 \mathrm{~nm}$ with the current laser diode. This infrared radiation can be used in frequency mixing. In this work, frequency doubling of the IR signal as well as sum-frequency generation with the second or fourth harmonic if the Nd:YAG laser was performed. Both OPOs were pumped by the second harmonic of the same Nd:YAG laser and it was thus possible to perform the pump as well as the dump step in the optical preparation of high vibrational states (section 5) with the same Nd:YAG radiation source.

\subsubsection{Dye laser}

A Nd:YAG (PRO-270, Spectra Physics) pumped dye laser (Sirah, Precision scan, PRSC-DA-24) was used for the sweep step in the optical preparation of high vibrational states. The bandwidth of $\approx 0.1 \mathrm{~cm}^{-1}$ is achieved via a holographic grating (2400 lines $/ \mathrm{mm}$, 1st order) at grazing incidence. The laser was typically operated with a solution of $\mathrm{C}-450$ (Exciton, coumarin dye) in methanol to generate radiation in the range between $445 \mathrm{~nm}$ and $455 \mathrm{~nm}$.

\subsubsection{Sunlite Ex OPO with FX-1 UV frequency extension}

In order to be able to perform scans over very broad wavelength ranges to probe many scattered vibrational states of the NO molecule via REMPI spectroscopy, an OPO laser system (Continuum, Sunlite Ex) pumped by the third harmonic of a Nd:YAG laser (Continuum, Powerlite DLS 9010) was purchased in 2012. Similar to the dye laser, this all solid-state laser uses a holographic grating to achieve its linewidth and thus achieves approximately the same bandwidth $\left(0.1 \mathrm{~cm}^{-1}\right)$. The OPO signal (445 to $710 \mathrm{~nm})^{1}$ or alternatively the OPO idler $(710$ to $1850 \mathrm{~nm})$ can be used directly or alternatively frequency doubled in a frequency conversion unit (Continuum, FX-1 conversion unit).

\footnotetext{
${ }^{1}$ The lowest wavelength of $445 \mathrm{~nm}$ corresponding to $225.5 \mathrm{~nm}$ after frequency doubling is the specification given by the company. In daily use, the OPO works stable at wavelengths above $235 \mathrm{~nm}$.
} 


\subsection{Data acquisition and processing}

Many parts of the setup are controlled by virtual instruments (VIs) programmed in LABVIEW. For example, these VIs start and stop scans of the laser systems, control the temperature of the surface and read out the oscilloscopes (LeCroy Waverunner LT344 and Tectronix DPO 4104), which get signals from the micro channel plate detector and the photomultiplier tube. In addition, laser powers are constantly monitored. The frequencies of the lasers are measured with a wavemeter (WS7, High Finesse GmbH) using fiber optics for light transport and a multi-channel switch (High Finesse GmbH, MC8) allowing for the simultaneous measurement of up to eight different frequencies ${ }^{1}$ Using this wavemeter, the frequencies of the home-built OPO lasers as well as the IR light source were locked to chosen frequencies for optical pumping.

The temporal delays in the experiments - including the pulsing of the piezo nozzle for the supersonic jet expansion, the firing of flash lamps and Q-switches of the Nd:YAG lasers as well as the pulsing of the MCP detector and the electric field - are controlled via four delay generators (Stanford Research Systems, DG535). The relevant time delays are explained in Appendix C.

\footnotetext{
${ }^{1}$ It is important to use this multi-channel switch at the lowest possible pulsed laser power. The switch is designed for cw lasers rather than for pulsed ns-lasers. The laser power should be such that the wavelength can only be read out after approx. 10 laser pulses ( 1 second acquisition time), otherwise the channels break. The wavemeter itself is rather robust. The correct procedure is to couple the light into the wavemeter $(<100 \mu \mathrm{J})$, adjust the laser power and only then to couple light into the fiber switch.
} 
3. Experimental 


\section{4}

\section{Orienting polar molecules without hexapoles}

As described in section 2.4.3, a major prediction of theoretical work regarding the "Huang experiment" was that the vibrational relaxation of $\mathrm{NO}$ scattering from $\mathrm{Au}(111)$ should be strongly dependent on the molecular orientation with respect to the surface. While orienting a molecule in an $a b$ initio calculation is relatively easy (it is a matter of defining starting parameters in a computer code or selecting specific trajectories after the calculation), achieving control over a molecules orientation in an experimental way is much more challenging.

All of the results for the investigation of oriented molecules in surface dynamics I reviewed in section 2.5 used an orientation method based on a state selection with an electrostatic hexapole(98) and subsequent orientation in an electric field.(99). However, this traditional approach has some limitations. Among others, the technique is - at least for molecules with a small dipole moment such as NO - limited to molecular beams with low translational energy.

We thus developed an alternative orientation technique in which the hexapole state selection is replaced by an optical state selection.(3) This technique is smaller and simpler than the hexapole approach and works over a wide range of translational energies. With respect to our goal of preparing not only oriented but at the same time vibrationally excited NO molecules, the approach is even more advantageous, because optical pumping is a necessary step anyhow.

In this chapter I will first review the theory of orienting molecules in molecular beams. 


\section{Orienting polar molecules without hexapoles}

I will then compare the new orientation method with the traditional approach and furthermore demonstrate the successful implementation of the new method using the example of orienting the $\mathrm{NO}$ molecule in its $\mathrm{X}^{2} \Pi_{1 / 2}(v=16, J=0.5)$ state.

\subsection{Theory}

A symmetric top molecule - or a molecule with a rotational wave function described by a linear combination of symmetric top wave functions such as NO in its $\mathrm{X}^{2} \Pi$ state — with an electric dipole moment $\boldsymbol{\mu}$ will be oriented when introduced into an electric field. A molecule with an electric dipole moment $\boldsymbol{\mu}$ can interact with an electric field with the electric field strength $\boldsymbol{E}$ via the linear Stark effect. If the external electric field is much smaller than the internal electric field of the electron-nuclei interaction (which is usually the case), this can be treated with first order perturbation theory with the operator $(100,101,102)$

$$
\hat{H}_{\text {Stark }}^{(1)}=-\boldsymbol{\mu} \cdot \boldsymbol{E}=-\mu_{\mathrm{Z}} E_{\mathrm{Z}} \cos \theta=-|\boldsymbol{\mu}||\boldsymbol{E}| \cos \theta
$$

where we define the electric field to act along the space-fixed $Z$-axis. By symmetry, the dipole moment of a molecule must lie along the molecule fixed $z$-axis. The angle $\theta$ is the angle between the dipole moment and the electric field and at the same time the Euler angle $\theta$ (see Appendix A.3.

We now consider a symmetric top wave function with the rotational quantum number $J$ (total angular momentum) that makes a projection $\Omega$ on the body fixed $z$-axis and a projection $M$ on the space fixed $Z$-axis (see equation A.29 and Fig. A.1 in the appendix)

$$
\psi_{J \Omega M}(\phi, \theta, \chi)=|J \Omega M\rangle=(-1)^{\Omega-M}\left[\frac{2 J+1}{8 \pi^{2}}\right]^{\frac{1}{2}} D_{-\Omega-M}^{J}(\phi, \theta, \chi),
$$

where $D_{\Omega M}^{J}(\phi, \theta, \chi)$ are the elements of the $(2 J+1) \times(2 J+1)$ Wigner rotation matrix, and $\theta, \chi$, and $\phi$ are the Euler angles. The Stark operator acts on a symmetric top wave function as

$$
\begin{aligned}
\Delta W_{\mathrm{Stark}}^{(1)} & =\left\langle J \Omega M\left|\hat{H}_{\mathrm{Stark}}^{(1)}\right| J \Omega M\right\rangle \\
& =-|\boldsymbol{\mu}||\boldsymbol{E}| \int_{0}^{2 \pi} \int_{0}^{\pi} \int_{0}^{2 \pi} \psi_{J \Omega M}^{*}(\phi, \theta, \chi) \cos \theta \psi_{J \Omega M}(\phi, \theta, \chi) d \phi \sin \theta d \theta d \chi \\
& =-|\boldsymbol{\mu}||\boldsymbol{E}|\langle\cos \theta\rangle
\end{aligned}
$$


where integration yields that the expectation value for the cosine of the orientation angle $\theta$ is given by

$$
\langle\cos \theta\rangle=\frac{M \Omega}{J(J+1)} .
$$

In order to describe rotational energy levels of a symmetric top molecule, it is necessary to define stationary states of defined parity (eigenfunctions of the inversion operator and the rotational Hamiltonian), which are constructed as linear combinations of symmetric top wave functions

$$
|J,| \Omega|, M, \pm\rangle=\frac{1}{\sqrt{2}}(|J, \Omega, M\rangle+\epsilon|J,-\Omega, M\rangle),
$$

where $\epsilon= \pm 1$ gives the parity of the state, labelled with $(e)$ and $(f)$ according to the convention of $e(\epsilon=+1)$ and $f(\epsilon=-1)$.

Elements of the linear Stark effect $\hat{H}_{\text {Stark }}^{(1)}$ are only non-zero for states of opposite parity:(102)

$$
\left\langle J \Omega M \pm\left|\hat{H}_{\text {Stark }}^{(1)}\right| J \Omega M \mp\right\rangle=-|\boldsymbol{\mu}||\boldsymbol{E}| \frac{M \Omega}{J(J+1)} .
$$

In the $\mathrm{X}^{2} \Pi_{1 / 2}$ ground state of the NO molecule, rotational levels belonging to the same $J$ are in the absence of an electric field split by the $\Lambda$-splitting $E_{\Lambda}$. These states of opposite parity of the $\Lambda$-doublet are those that first mix at low electric field strength, as they are close in energy.(102) Thus, we can write an effective $2 \times 2$ Hamiltonian of the form

$$
H=\left(\begin{array}{cc}
\frac{E_{\Lambda}}{2} & -|\boldsymbol{\mu}||\boldsymbol{E}| \frac{M \Omega}{J(J+1)} \\
-|\boldsymbol{\mu}||\boldsymbol{E}| \frac{M \Omega}{J(J+1)} & \frac{-E_{\Lambda}}{2}
\end{array}\right) .
$$

This Hamiltonian has the eigenvalues

$$
U= \pm \sqrt{\left(\frac{E_{\Lambda}}{2}\right)^{2}+\left(|\boldsymbol{\mu}| \frac{M \Omega}{J(J+1)}|\boldsymbol{E}|\right)^{2}}
$$

and the eigenfunctions

$$
\left|\Psi_{ \pm}\right\rangle_{E}=\frac{1}{\sqrt{\alpha(E)^{2}+\beta(E)^{2}}}(\alpha(E)|J,| \Omega|, M,+\rangle \pm \beta(E)|J,| \Omega|, M,-\rangle)
$$

The mixing coefficients $\alpha_{E}$ and $\beta_{E}$ strongly depend on the reduced electric field strength 


\section{Orienting polar molecules without hexapoles}

$E_{\mathrm{red}}=2 \Delta W_{\text {Stark }}^{(1)} / E_{\Lambda}$, meaning the ratio of the Stark energy relative to the $\Lambda$-splitting.

$$
\begin{aligned}
& \alpha(E)=\sqrt{\frac{1}{2}+\frac{1}{2 \sqrt{1+E_{\mathrm{red}}^{2}}}} \\
& \beta(E)=\sqrt{\frac{1}{2}-\frac{1}{2 \sqrt{1+E_{\mathrm{red}}^{2}}}}
\end{aligned}
$$

In case of a strong electric field, the mixing coefficients approach $\alpha(E)=\beta(E)=\frac{1}{\sqrt{2}}$ and the wave functions reduce to

$$
\left|\Psi_{ \pm}\right\rangle_{E \rightarrow \infty}=\frac{1}{\sqrt{2}}(|J,| \Omega|, M,+\rangle \pm|J,| \Omega|, M,-\rangle) .
$$

The degree of orientation is finally obtained by calculating the expectation value $\langle\cos \theta\rangle$ for the wave function as given in equation 4.9, which can be evaluated to (103)

$$
\langle\cos \theta\rangle=2 \alpha(E) \beta(E)\langle\cos \theta\rangle_{\max },
$$

where

$$
\langle\cos \theta\rangle_{\max }=\langle\cos \theta\rangle_{E \rightarrow \infty}=\frac{M \Omega}{J(J+1)}
$$

is the expectation value for the cosine of the orientation angle in the limit of a complete mixing of the opposite parity rotational states due to the Stark effect. Thus, $2 \alpha(E) \beta(E)$ is a measure representing the degree of orientation on a scale between 0 and 1 . A value of 0 means no orientation while a value of 1 corresponds to the maximum possible orientation for a given quantum state. By combining equations $4.10,4.11$ and 4.6 we obtain:

$$
2 \alpha(E) \beta(E)=2 \sqrt{\frac{\left(|\boldsymbol{\mu} \| \boldsymbol{E}|\langle\cos \theta\rangle_{\max }\right)^{2}}{E_{\Lambda}^{2}+4\left(|\boldsymbol{\mu} \| \boldsymbol{E}|\langle\cos \theta\rangle_{\max }\right)^{2}}}=2 \sqrt{\frac{\left(|\boldsymbol{\mu} \| \boldsymbol{E}| \frac{M \Omega}{J(J+1)}\right)^{2}}{E_{\Lambda}^{2}+4\left(|\boldsymbol{\mu} \| \boldsymbol{E}| \frac{M \Omega}{J(J+1)}\right)^{2}}} .
$$

This means, that the degree of orientation is a function of the electric field strength, the $\Lambda$-splitting, the dipole moment and the quantum numbers $J, \Omega$ and $M$. Fig. 4.1 shows how the Stark splitting and the degree of orientation evolve as a function of the electric field strength for NO molecules in their electronic and vibrational ground state $X^{2} \Pi_{1 / 2}(v=0, J=0.5)$. In its vibrational ground state the $\mathrm{NO} \mathrm{X}^{2} \Pi_{1 / 2}(v=0)$ molecule has a $\Lambda$-splitting of $E_{\Lambda}=0.012 \mathrm{~cm}^{-1}(104)$ and a dipole moment of -0.159 Debye $\left(\mathrm{N}^{-} \mathrm{O}^{+}\right.$polarity)(105). 


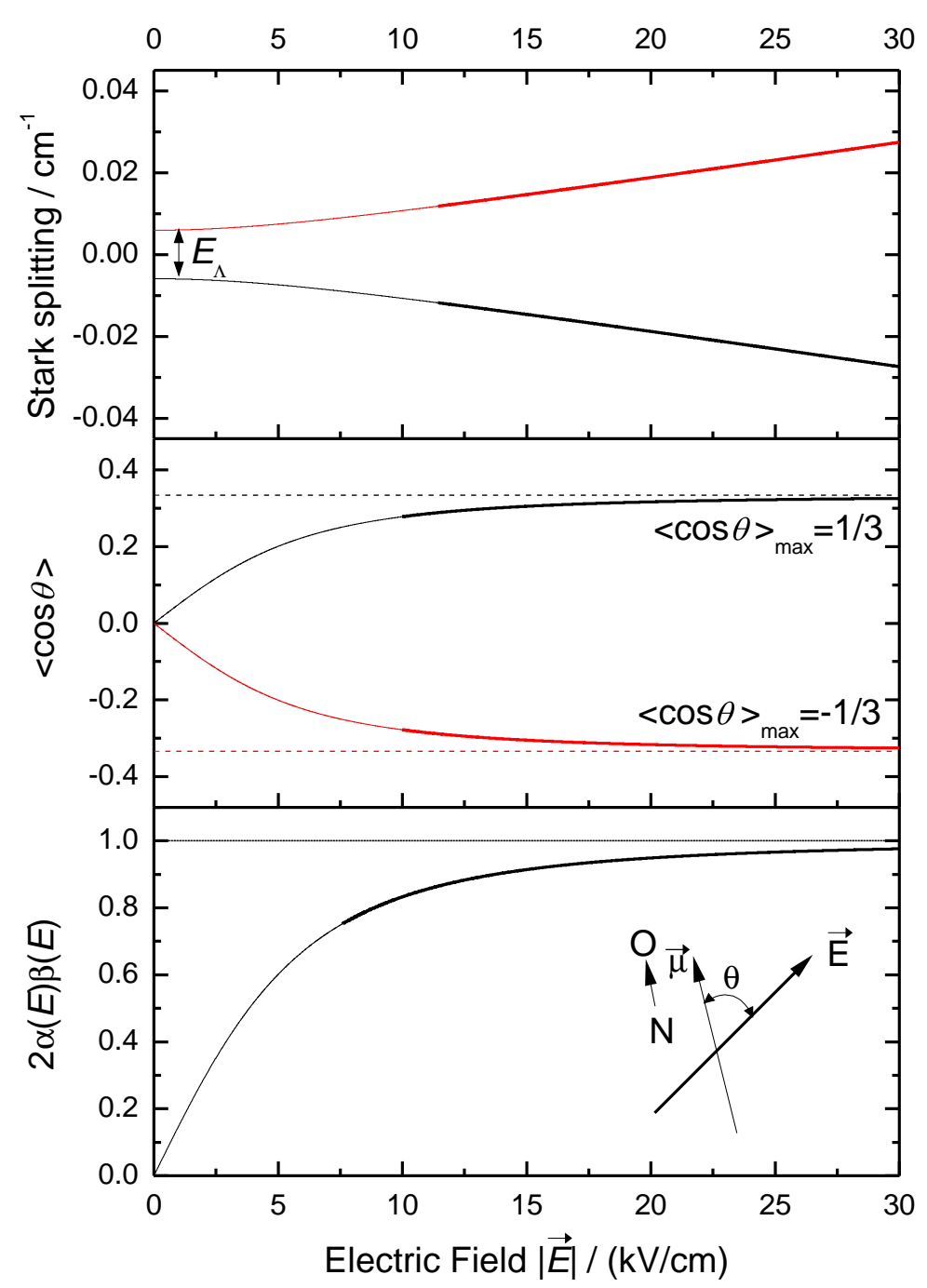

Figure 4.1: Stark effect and orientation in the electronic ground state of the NO molecule - (top) Energy separation of the $(e)$ and $(f)$ parity states (black and red color, respectively) of $\mathrm{NO} \mathrm{X}^{2} \Pi_{1 / 2}(v=0, J=0.5)$ as function of the electric field strength. The energy is calculated according to equation 4.8 with a $\Lambda$-splitting of $E_{\Lambda}=0.012 \mathrm{~cm}^{-1}$ and a dipole moment -0.159 Debye $\left(\mathrm{N}^{-} \mathrm{O}^{+}\right.$parity). (middle) Expectation values for the cosine of the orientation angle $\langle\theta\rangle$ for the two parity states as calculated with equation 4.13 . The states evolve adiabatically into oriented states in the electric field. At higher field strength, the value $\langle\cos \theta\rangle_{\max }= \pm 1 / 3$ is approached (shown as dashed lines). (bottom) The degree of orientation can also be described in terms of the quantity $2 \alpha(E) \beta(E)$ as calculated by equation 4.15 . This value approaches the value 1 when the maximum possible orientation is achieved. The sketch in the panel shows the orientation angle $\theta$, which is the angle between the dipole moment (lying along the bond axis) and the electric field and at the same time the Euler angle used for rotating a space-fixed to a molecule-fixed coordinate system. Electric field strength of $30 \mathrm{kV} / \mathrm{cm}$ (maximum of the shown plot) can be easily generated with high voltage electrodes, if great care is taken to avoid voltage flashovers. 


\section{Orienting polar molecules without hexapoles}

At electric field strength of $20-30 \mathrm{kV} / \mathrm{cm}$ the maximum degree of orientation for these states $\langle\cos \theta\rangle_{\max }= \pm 1 / 3$ is approached $(J=|\Omega|=|M|=0.5)$. This corresponds to values for the orientation angle of $\cos ^{-1}(1 / 3)=70.5^{\circ}$ and $\cos ^{-1}(-1 / 3)=109.5^{\circ}$, respectively. Alternatively, it is also possible to calculate the expectation values $\langle\theta\rangle_{\max }$, which give values of $67.5^{\circ}$ and $112.5^{\circ}$ for the different parity states, respectively.

\subsection{The traditional approach: Orientation via hexapole state selection}

So far it has been shown, that opposite parity states of symmetric top molecules, or molecules described by symmetric top wave functions such as the $X^{2} \Pi_{1 / 2}(v=0, J=$ $0.5)$ state of NO, can be oriented in an electric field. However, the different parity states are oriented in opposite direction. As these states are close in energy (which they need to be for effective mixing in the electric field via the Stark effect), they are thermally or even after a supersonic jet expansion almost equally populated. This means, that a net orientation can only be achieved in case a single parity state is prepared or filtered. In the traditional approach this is done via state selection with a hexapole, see Fig. 4.2 . Hexapole state selection uses an inhomogenous electric field created by the alternating

(a)

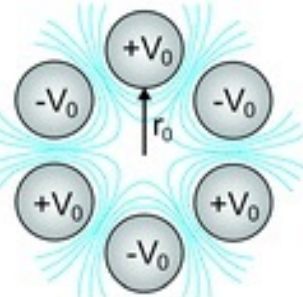

(b)

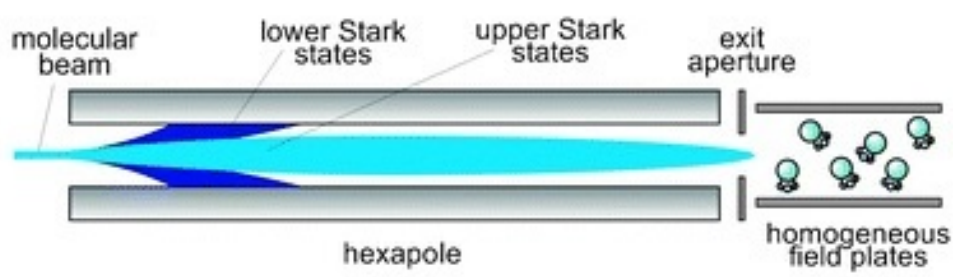

Figure 4.2: Classical orientation approach using a hexapole. - (left) The hexapole creates an inhomogeneous electric field. (right) The hexapole refocusses upper Stark states of the molecular beam and defocuses the other Stark states. The state selected molecular beam is then introduced into a homogeneous electric field and an the polar molecules are oriented. Reproduced from Ref. (106) with permission of The Royal Society of Chemistry.

positive and negative voltages applied to the six rods of the hexapole. It can be shown, that Stark states which are stabilized in energy due to the Stark effect (lower Stark states, which are also called high-field seeking) are always deflected. As opposed to this, for molecules in the upper Stark state (the one increasing in energy in the electric 
field, low-field seeking state) the electric field in the hexapole exerts a force toward the propagation axis. Due to the length of the hexapole it is necessary to refocus molecules of the low-field seeking state of a divergent molecular beam (molecules have a small velocity component perpendicular to the beam propagation) at the end of the hexapole. This is achieved when the condition(99)

$$
l_{\text {hex }}=\sqrt{\frac{\pi^{2} r_{0}^{3}}{3} \frac{J(J+1)}{M \Omega} \frac{0.5 m v^{2}}{|\boldsymbol{\mu}| V_{0}}} \propto \sqrt{\frac{\text { kinetic energy }}{\text { Stark energy }}}
$$

is fulfilled, where $l_{\text {hex }}$ is the length of the hexapole, $m$ is the mass of the molecule and $v$ is its velocity. $V_{0}$ is the voltage of the hexapole (alternating $(+)$ and (-)-polarity on the rods of the hexapole) and $r_{0}$ is the distance between the hexapoles axis of symmetry and the rods. As a consequence, the hexapole only refocuses molecules of a single velocity and a velocity spread in the molecular beam rapidly reduces the state resolving power of the hexapole. Thus, orientation experiments may require using a velocity selector.(98, 107) In addition, as the Stark energy is typically small $\left(10^{-4} \mathrm{eV}\right.$, depending on the dipole moment), the length of the hexapole required for refocussing increases unfavourably with the kinetic energy. For the NO molecule in its electronic and vibrational ground state $\mathrm{X}^{2} \Pi_{1 / 2}(J=0.5)$ the typical length of the hexapole $(1 \mathrm{~m})$ can only refocus relatively slow beams with a kinetic energy smaller than $0.4 \mathrm{eV}$. Refocussing a beam with an increased kinetic energy by a factor of 2.5 (to achieve $1 \mathrm{eV}$ ) would require an increased length of the hexapole by a factor of $2.5^{2}=6.25$.

\subsection{The new approach: Optical state--selection with adi- abatic orientation}

We developed an alternative approach to the orientation of polar molecules, where we replace hexapole state selection with an optical state selection using optical pumping to excited single parity states energetically split at zero electric field (e.g. the $\Lambda$-splitting separating $(e)$ and $(f)$ parity states in $\left.\mathrm{NO} \mathrm{X}^{2} \Pi_{1 / 2}\right)$. Similar to the classical approach, the state-selected molecules then enter a region of a static electric field (e.g. between a high voltage electrode and a grounded surface) and evolve adiabatically into oriented states, see Fig. 4.3. The concept was inspired by previous work, where lasers were used 


\section{Orienting polar molecules without hexapoles}

to optically excite specific $M$-states, whose degeneracy was broken by the external electric field to orient $\mathrm{CO}$ molecules in the metastable $a^{3} \Pi$ state.(108)

For example molecules in the third excited vibrational state of NO $X^{2} \Pi_{1 / 2}(v=3, J=$

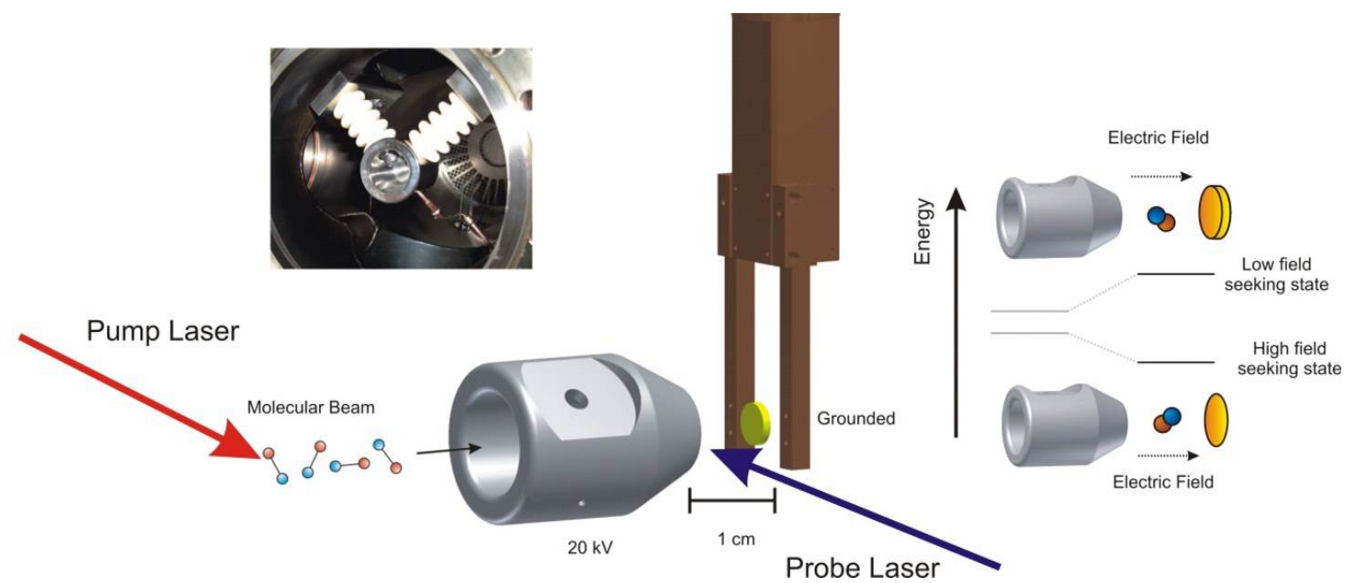

Figure 4.3: Optical state selection with adiabatic orientation - Optically excited molecules of single parity fly through an orientation electrode into a region of a static electric field (between the orientation electrode and the grounded surface). The selected states evolve adiabatically into orientated states. This way it is possible to orient the NO molecule preferentially with the $\mathrm{O}$-atom or with the $\mathrm{N}$-atom towards the surface.

$0.5)$ can be parity selectively $((e)$ or $(f)$-parity) prepared via vibrational overtone pumping with the Fourier-transform limited infrared laser source. The initial preparation method (hexapole vs optical state selection) does not influence the final molecular orientation. Thus both methods generate the same orientation distributions.

The approach using optical state selection has advantages and disadvantages compared to the hexapole state selection, which I would like to briefly discuss.

The main drawback of the optical approach is that only those molecules excited by the optical preparation are oriented and other states are not filtered out. For example, the molecular beam expansion of NO in this work not only produces molecules in the electronic and vibrational ground state $\mathrm{NO} \mathrm{X}^{2} \Pi_{1 / 2}(v=0, J=0.5)$, but also in other rotational states (approx. 50\%, mostly in $v=1.5$ and $2.5(\overline{96})$ ) in the other spin-orbit state $\mathrm{X}^{2} \Pi_{3 / 2}(v=0)$ (approx. $\left.35 \%\right)$ and in $\mathrm{X}^{2} \Pi_{\Omega}(v=1)$ (approx. 0.01\%). In addition, population in $\mathrm{X}^{2} \Pi_{1 / 2}(v=0)$ is equally divided (the energy splitting is small) into the two different parity components. Only a fraction of one of these components is opti- 
cally excited to a higher vibrational state and the excited molecules will have a net orientation.

As opposed to this, the hexpole technique can create molecular beams consisting exclusively out of one quantum state, as other ro-vibrational or spin-orbit states are defocused by the hexapole. In addition, the carrier gas, - which typically does not have a dipole moment and is thus not affected by the Stark effect - can be blocked by introducing a beam dump on axis in the center of the hexapole.(109)

The most important advantage of the optical state selection is that it works independently of the velocity and the velocity spread of the molecular beam. The only criterion that has to be considered is that the energy ramp of the electric field has to be slow enough not to introduce non-adiabatic transitions between the high and low-field seeking states. This condition is however easily satisfied (3, 102). In reaction dynamics, studying oriented molecules at high translational energy can be particularly interesting, when long-range reorienting forces (steering effects (5, 110, 111, 112, 113, 114)) are involved. Here, faster molecules are less likely to become reoriented than slow molecules, as the interaction time with the surface is reduced.

Another advantage is that the optical approach is smaller and simpler. In particular for experiments where an optical excitation of molecules is desired anyhow, it makes sense to avoid using a hexapole. As the laser excitation requires little space, it is possible to keep the distance between the nozzle and the surface in a molecular beam surface scattering experiment short, which is important to achieve high beam densities(91) (although this effect is partially compensated in the hexapole approach due to the refocusing effect).

A third advantage of the optical approach is that both the high-field and the low-field seeking state can be optically prepared, which the hexapole will always transmit the low-field seeking state only. This means, that the orientation can be optically flipped by changing the excitation wavelength.(4)

Although we developed the novel orientation technique with the specific goal of orienting vibrationally excited NO molecules, it should also be extendible for orienting other molecules (see Ref. (3) for a detailed discussion). The orientation method requires a symmteric top molecule or molecules described as symmetric tops (e.g. NO in the $\mathrm{X}^{2} \Pi$ state or $\mathrm{CO}$ in the $\mathrm{a}^{3} \Pi$ state) or near-symmetric (asymmetric) tops (e.g. $\mathrm{H}_{2} \mathrm{CO}$ ). In 


\section{Orienting polar molecules without hexapoles}

addition, an energetic separation of different parity states and an electric dipole moment are necessary. In case the energy separation is too small to be resolved under field free conditions, there is always the opportunity of performing the optical preparation within the electric Stark field.

\subsection{Testing the method and determining the achieved de- gree of orientation}

After developing the concept of the new orientation technique with the optical excitation, it was successfully implemented in our laboratory. (3)

It was tested for NO molecules prepared parity selectively $((e)$ or $(f)$-parity) in a high vibrational state $\mathrm{X}^{2} \Pi_{1 / 2}(v=16, J=0.5)$ by stimulated emission pumping (SEP) via the $\mathrm{B}^{2} \Pi_{1 / 2}(v=3)$ state, see Fig. 4.4 The experimental setup was as follows: a pulsed supersonic molecular beam of rotationally cold NO molecules $\left(T_{\text {rot }}=6 \mathrm{~K}\right)$ was generated by expanding a mixtures of either $60 \% \mathrm{NO} / \mathrm{Kr}\left(E_{\text {trans }}=0.035 \mathrm{eV}\right)$ or $1 \% \mathrm{NO} / \mathrm{H}_{2}$ $\left(E_{\text {trans }}=1.0 \mathrm{eV}\right)$ into vacuum. In the differential chamber molecules were prepared in either of the parity states $\left((e)\right.$ or $(f)$-parity) of $\mathrm{X}^{2} \Pi_{1 / 2}(v=16, J=0.5)$ by excitation with one of the home-build OPO laser systems via the $\mathrm{B}^{2} \Pi_{1 / 2}\left(v^{\prime}=3\right) \leftarrow \mathrm{X}^{2} \Pi_{1 / 2}\left(v^{\prime \prime}=\right.$ $0) \mathrm{R}_{11}(0.5)$ (PUMP) transition at $206.15 \mathrm{~nm}$ followed by de-excitation with the second harmonic of a dye laser via the $\mathrm{B}^{2} \Pi_{1 / 2}\left(v^{\prime}=3\right) \rightarrow \mathrm{X}^{2} \Pi_{1 / 2}\left(v^{\prime \prime}=16\right) \mathrm{R}_{11}(0.5)$ (DUMP) transition at $458.1 \mathrm{~nm}$. The NO molecular beam then entered the surface chamber and passed along the symmetry axis through the cylindrical, $7 \mathrm{~cm}$ long, orientation electrode. In the region between the electrode and the grounded surface (in this particular experiment the electrode-surface distance was $1 \mathrm{~cm}$ ) electric fields of up to $18 \mathrm{kV} / \mathrm{cm}$ were generated. In the region of the electric field, $\mathrm{NO}(v=16)$ was detected by laser induced fluorescence (LIF) spectroscopy via the $\mathrm{A}^{2} \Sigma^{+}$state 1.1 .

Panels a) and c) of Fig. 4.5 show LIF spectra in the absence of an electric field for molecules prepared either in the $(e)$ or $(f)$-parity state of $\mathrm{X}^{2} \Pi_{1 / 2}(v=16, J=0.5)$, respectively. The LIF spectra mainly show the transitions allowed due to selection rules, which are the unresolved $\mathrm{R}_{11}(0.5) / \mathrm{Q}_{21}(0.5)$ line in panel a) and the $\mathrm{R}_{21}(0.5)$ and

\footnotetext{
${ }^{1}$ LIF spectroscopy used a photomultiplier tube (PMT) in the surface chamber. The PMT has not been mentioned in the experimental section of this thesis, because it was later replaced by the micro-channel plate detector for the surface scattering experiments.
} 


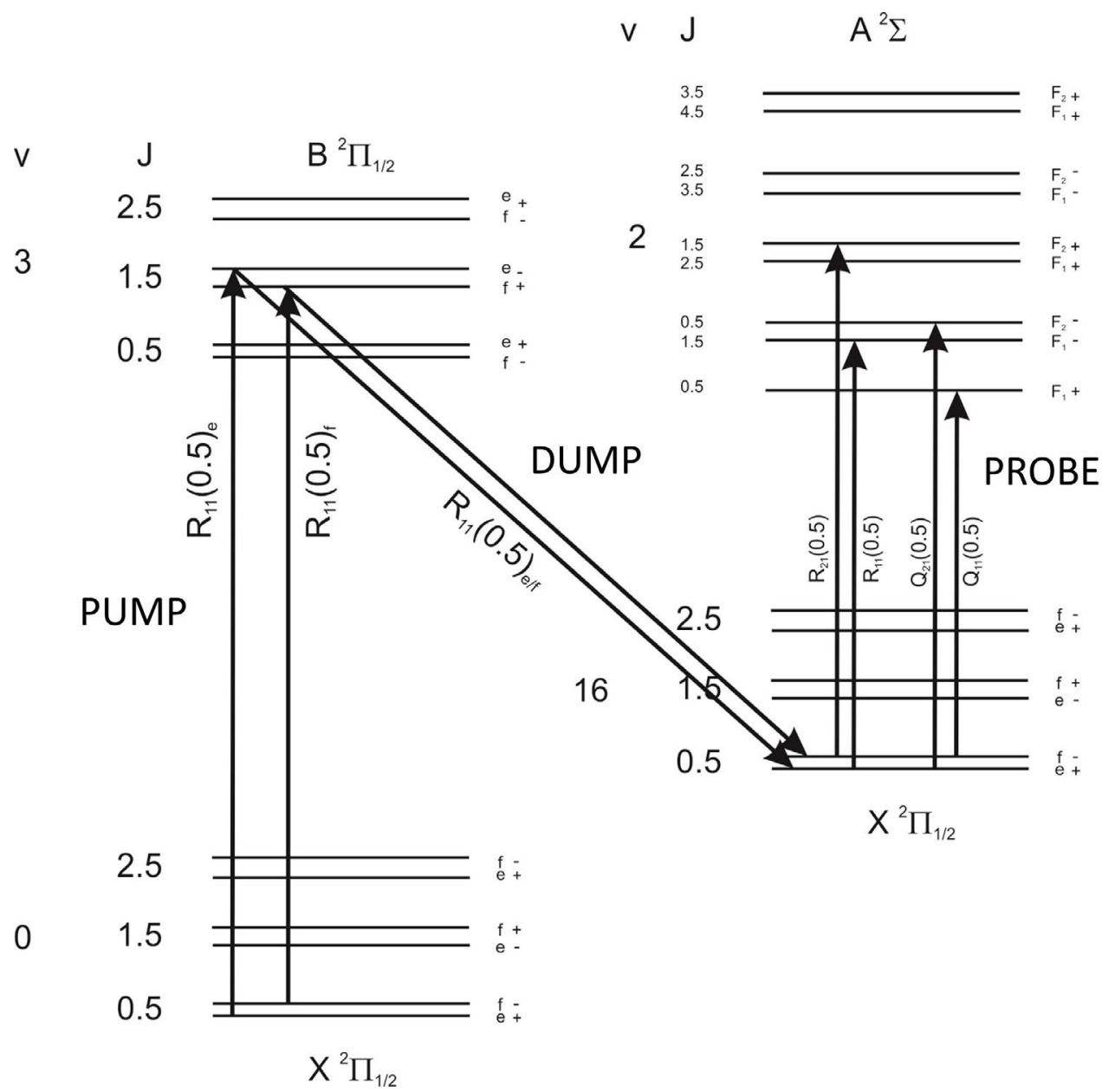

Figure 4.4: Energy diagram explaining the spectroscopy of the orientation experiment - In the differential chamber molecules were prepared in either of the parity states $\left((e)\right.$ or $(f)$-parity) of $\mathrm{X}^{2} \Pi_{1 / 2}(v=16, J=0.5)$. This was done via excitation with one of the home-built OPO laser systems via the $\mathrm{B}^{2} \Pi_{1 / 2}\left(v^{\prime}=3\right) \leftarrow \mathrm{X}^{2} \Pi_{1 / 2}\left(v^{\prime \prime}=0\right) \mathrm{R}_{11}(0.5)$ (PUMP) transition followed by de-excitation via the $\mathrm{B}^{2} \Pi_{1 / 2}\left(v^{\prime}=3\right) \rightarrow \mathrm{X}^{2} \Pi_{1 / 2}\left(v^{\prime \prime}=\right.$ 16) $\mathrm{R}_{11}(0.5)$ (DUMP) transition. The resolution of the PUMP laser $(250 \mathrm{MHz})$ is sufficient to pump either the $\mathrm{R}_{11}(0.5)_{e}$ or the $\mathrm{R}_{11}(0.5)_{f}$ transition. Due to the parity selection rule

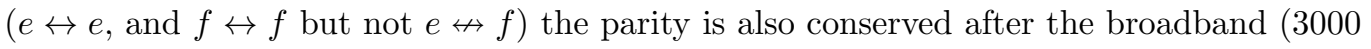
$\mathrm{MHz})$ DUMP transition. Population in $\mathrm{X}^{2} \Pi_{1 / 2}\left(v^{\prime \prime}=16, J=0.5\right)$ is then probed via laser induced fluorescence via the $\mathrm{A}^{2} \Sigma^{+}(v=2)$ state. Allowed transitions in zero electric field are labeled. The $\mathrm{R}_{11}(0.5)$ and $\mathrm{Q}_{21}(0.5)$ transitions originating from the $(e)$-parity state in $\mathrm{X}^{2} \Pi_{1 / 2}\left(v^{\prime \prime}=16, J=0.5\right)$ are not resolved by the probe laser (also $3000 \mathrm{MHz}$ ) due to the small spin-rotation interaction $\left(\gamma=2.718 \cdot 10^{-3} \mathrm{~cm}^{-1}\right)(104)$ separating the $\mathrm{F}_{1}$ and $\mathrm{F}_{2}$ levels in the $\mathrm{A}^{2} \Sigma^{+}(v=2)$ state. 


\section{Orienting polar molecules without hexapoles}

$\mathrm{Q}_{11}(0.5)$ lines in panel c). The spectra also show small contributions originating from the other parity states, due to imperfections in the optical preparation method 11 In case the electric field of $18 \mathrm{kV} / \mathrm{cm}$ is applied (panels b) and d)), the Stark effect mixes the states of different parity and zero-field forbidden transitions become allowed due to the mixing of zero field wave functions (intensity borrowing).

The transitions in the LIF spectra of Fig. 4.5 were not saturated. For example, the 2:1 intensity ratio of the $R_{11}(0.5)$ line to the $R_{21}(0.5)$ line in panel $d$ ) reflects the Hönl-London factors of these transitions. In this case, it is possible to derive the mixing coefficients $\alpha(E)$ and $\beta(E)$ by fitting the measured LIF spectra with appropriate Voigt profile functions (convolution of a Gaussian and a Lorentzian profile) and then comparing the spectral contributions of both parity states in the spectra with and without electric field (see Ref. (3) for details). As described in section 4.1, the mixing coefficients $\alpha(E)$ and $\beta(E)$ fully describe the degree of orientation of the molecule, which is often described on the $2 \alpha(E) \beta(E)$ scale. Fig. 4.5 shows the mixing coefficients $\alpha(E)^{2}$ and $2 \alpha(E) \beta(E)$ as function of the electric field strength. It is found, that at the maximum applied electric field strength of $18 \mathrm{kV} / \mathrm{cm}$ almost full orientation $\left(\langle\cos \theta\rangle>0.9\langle\cos \theta\rangle_{\max }\right)$ was achieved in the experiment and that the degree of orientation is independent of the kinetic energy of the NO molecules in the molecular beam. Furthermore, the measured orientation is in good agreement with how it should theoretically evolve as function of the electric field strength according to equation 4.15 (shown as black solid lines in Fig. 4.6).

\subsection{Orientation for different vibrational states in the NO molecule}

For NO molecules prepared in $v=16$, the electric field of $18 \mathrm{kV} / \mathrm{cm}$ was sufficient to almost fully saturate the degree of orientation $\left(\langle\cos \theta\rangle>0.9\langle\cos \theta\rangle_{\max }\right)$. However, as described by equation 4.15 , the strength of the electric field needed to achieve a certain orientation strongly depends on the $\Lambda$-splitting and the electric dipole moment. While the $\Lambda$-splitting is only weakly dependent on the vibrational state $\left(E_{\Lambda} \approx 0.12 \mathrm{~cm}^{-1}\right.$

\footnotetext{
${ }^{1}$ It should be pointed out, that these imperfections were not present in the scattering experiments using orientated beams, as molecules were later prepared in excited vibrational states with the pumpdump-sweep technique via the $\mathrm{A}^{2} \Sigma^{+}(v=2)$ state or via vibrational overtone pumping. Both of these techniques produce neglectful contributions of the other parity state.
} 

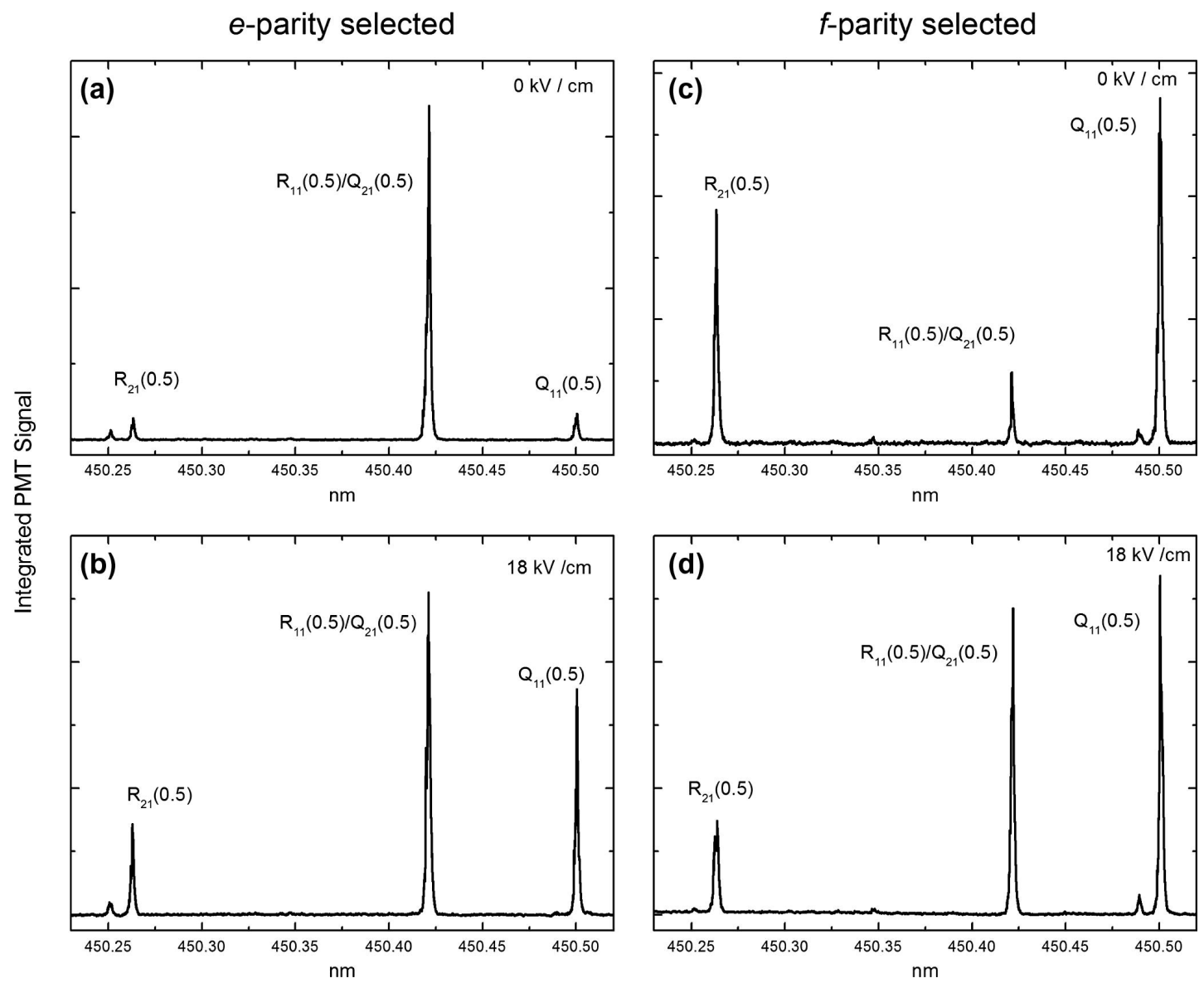

Figure 4.5: Mixing of opposite parity wavefunctions demonstrated by laser induced fluorescence spectroscopy - The figure shows LIF specta of the $\mathrm{A}^{2} \Sigma^{+}(v=$ $2) \leftarrow \mathrm{X}^{2} \Pi_{1 / 2}\left(v^{\prime \prime}=16\right)$ band in NO. In the left panels ((a) and (b) $)(e)-$ parity states have been prepared via stimulated emission pumping (SEP) and in the right panels ((c) and (d) $)(f)$-parity states have been prepared. In case an electric field of $18 \mathrm{kV} / \mathrm{cm}$ is applied (panels (b) and (c)) contributions of the other parity state are observed. This demonstrates the mixing of the opposite parity states of the $\Lambda$-doublet, meaning that the NO molecule is oriented in the laboratory frame. Reprinted from Publication (3), with permission from Elsevier. 


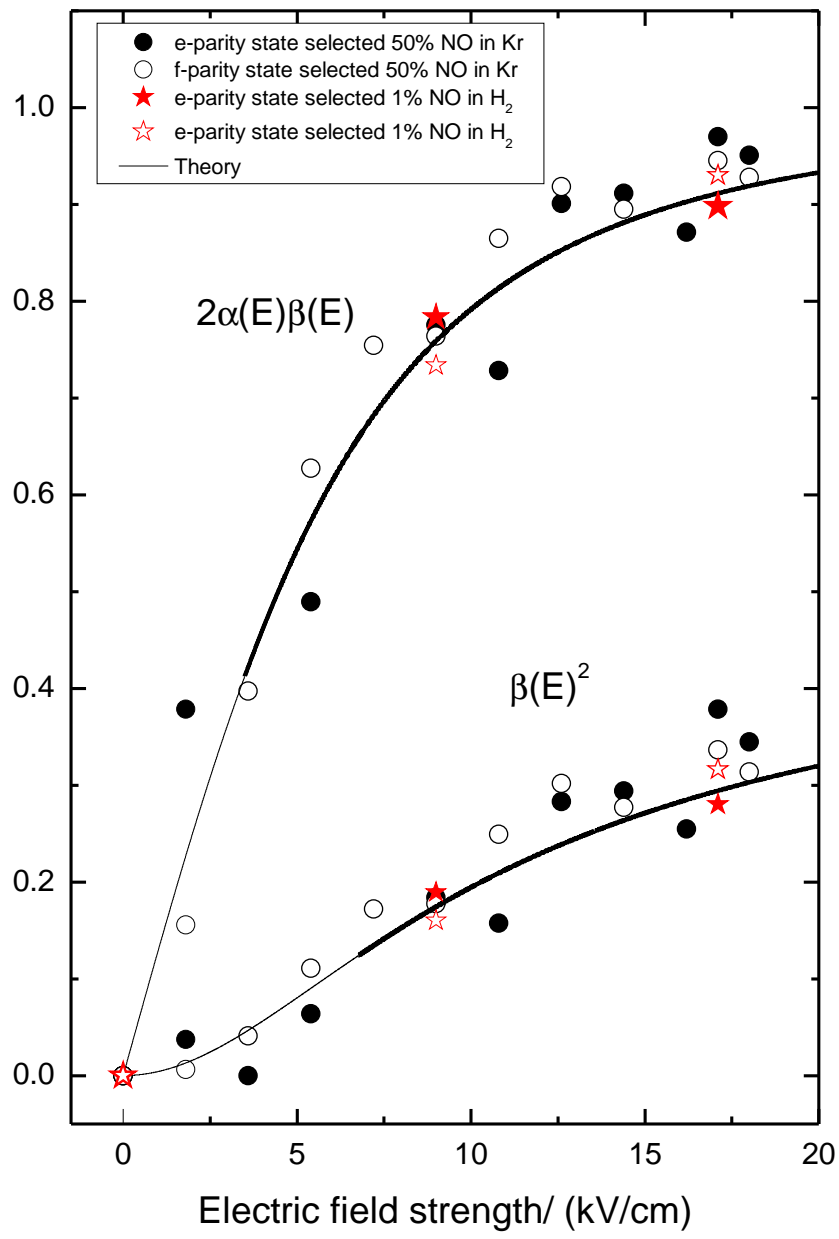

Figure 4.6: Degree of orientation as function of the electric field strength The degree of orientation $2 \alpha(E) \beta(E)$ and the square of the mixing coefficient $\beta(E)^{2}$ are plotted as function of the electric field strength for molecules prepared in $\mathrm{NO} \mathrm{X}^{2} \Pi_{1 / 2}(v=$ $16, J=0.5)$. Black solid circles denote $(e)$-parity state prepared molecules with $35 \mathrm{meV}$ translational energy, black open circles denote $(f)$-parity state prepared molecules with $35 \mathrm{meV}$ translational energy, red solid stars denote $(e)$-parity state prepared molecules with $1 \mathrm{eV}$ translational energy and red open stars denote $(f)$-parity state prepared molecules with $1 \mathrm{eV}$ translational energy. The black solid lines show calculated values from molecular constants according to equation 4.15, using the molecular constants $E_{\Lambda}=0.012 \mathrm{~cm}^{-1}$ and $|\boldsymbol{\mu}|=0.13887$ Debye. Reprinted from Publication (3), with permission from Elsevier. 
(104)), the dipole moment is strongly dependent on the nuclear separation and thus on the vibrational state. The dipole moment even changes sign from $\mathrm{N}^{-} \mathrm{O}^{+}$polarity at vibrational states below $v<9$ to $\mathrm{N}^{+} \mathrm{O}^{-}$polarity for $v \geq 9$, see Fig. 4.7.

We prepared NO $v=3,11$ and 16 molecules for surface scattering experiments (see
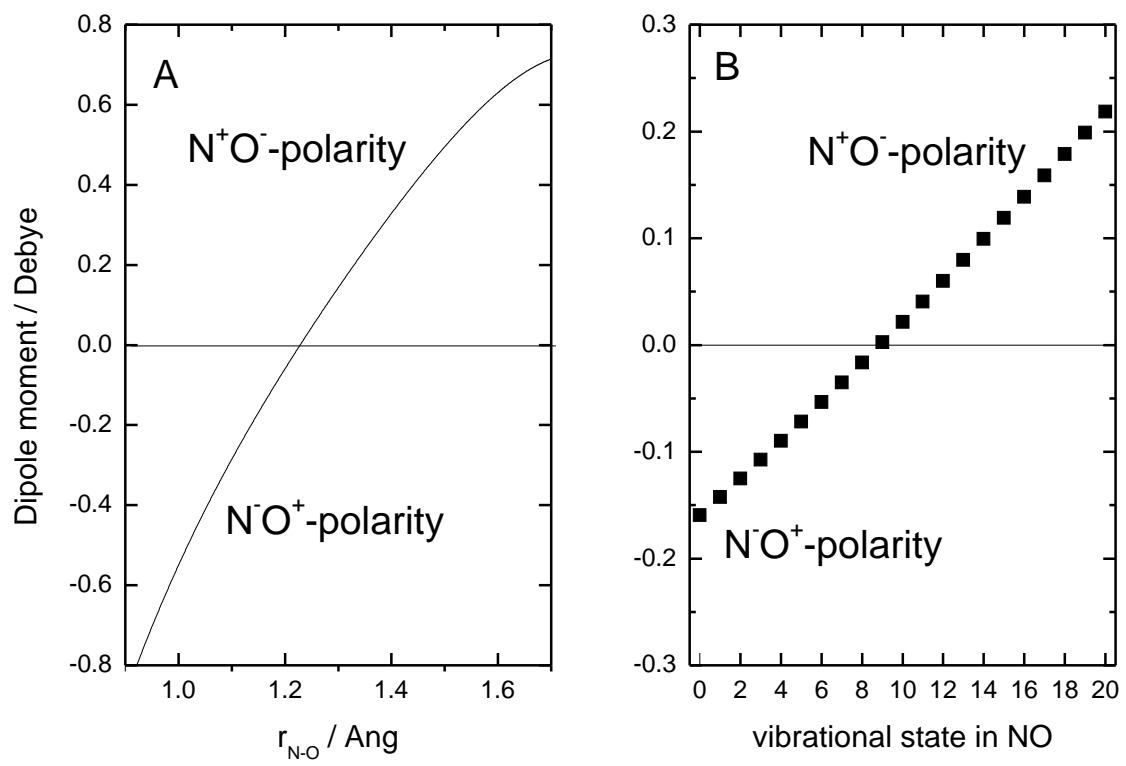

Figure 4.7: Dipole moment of nitric oxide - Dipole moment of nitric oxide $\mathrm{X}^{2} \Pi$ as function of the $\mathrm{N}-\mathrm{O}$ bond speration (panel a) and the vibrational state (panel b). The dipole moment was given in a functional form in Ref. (105).

chapter 6). Among these states, $v=11$ has by far the smallest dipole moment with 0.04064 Debye. Thus, while the first scattering experiments with $\operatorname{NO}(v=3)$ were performed with an electric field of $21.4 \mathrm{kV} / \mathrm{cm}$, the field strength was increased to $33 \mathrm{kV} / \mathrm{cm}$ for $v=11$ and 16 (which was the strongest field strength we managed to generate). Table 4.1 gives dipole moments $|\boldsymbol{\mu}|$, the $\Lambda$-splitting $E_{\Lambda}$ and the degree of orientation $2 \alpha_{e} \beta_{e}$ calculated for the different vibrational states at these electric field strengths. 
Table 4.1: Parameters relevant for calculating the degree of orientation in $\mathrm{NO} X^{2} \Pi_{1 / 2}(J=0.5)$ for different vibrational states $v$ with electric field strengths as used for the scattering experiments.

\begin{tabular}{ccccc}
\hline \hline$v$ & $|\boldsymbol{\mu}| /$ Debye $^{\mathrm{a})}$ & $E_{\Lambda} / \mathrm{cm}^{-1 \mathrm{~b})}|\boldsymbol{E}| /(\mathrm{kV} / \mathrm{cm})^{\mathrm{c})}$ & $\left.2 \alpha_{e} \beta_{e}^{\mathrm{d}}\right)$ \\
\hline 3 & -0.125 & 0.012 & 21.4 & 0.932 \\
11 & 0.041 & 0.011 & 33.3 & 0.800 \\
16 & 0.139 & 0.011 & 33.3 & 0.978 \\
\hline \hline
\end{tabular}
a) from Ref. (105).
b) from Ref. (104).
c) for $v=3: 15 \mathrm{kV}$ at $7 \mathrm{~mm}$ distance between electrode and surface, for $v=11$ and $16: 15 \mathrm{kV}$ at $4.5 \mathrm{~mm}$.
d) Calculated from equation 4.15 .

It is also possible to calculate the exact orientation distributions $P(\theta)$. These are obtained by integrating the square of the wavefunction over all Euler angles except for the orientation angle $\theta$

$$
P_{ \pm}(\theta)=\int_{0}^{2 \pi} \int_{0}^{2 \pi} \Psi_{ \pm}^{*} \Psi_{ \pm} d \phi \sin \theta d \chi
$$

For $J=|\Omega|=|M|=0.5$ this integral can be evaluated to

$$
P_{ \pm}(\theta)=0.5 \sin \theta \pm \alpha(E) \beta(E) \cos \theta \sin \theta
$$

and the orientation distributions are plotted in Fig. 4.8. At the electric fields applied for the surface scattering experiments, the orientation distributions are calculated to be close to the maximum possible orientation for the prepared quantum states. Nevertheless, a nominal $\mathrm{N}$-atom first orientation distribution does always also contain some molecules with the bond axis oriented with the $\mathrm{O}$-atom towards the surface and vice versa. A complete $\mathrm{N}$-atom or $\mathrm{O}$-atom end-on orientation is impossible due to the uncertainty principle in quantum mechanics. Knowledge of the orientation distributions as shown in Fig. 4.8 is important in order to perform quantitative comparisons between experimental data and $a b$ initio theory. 

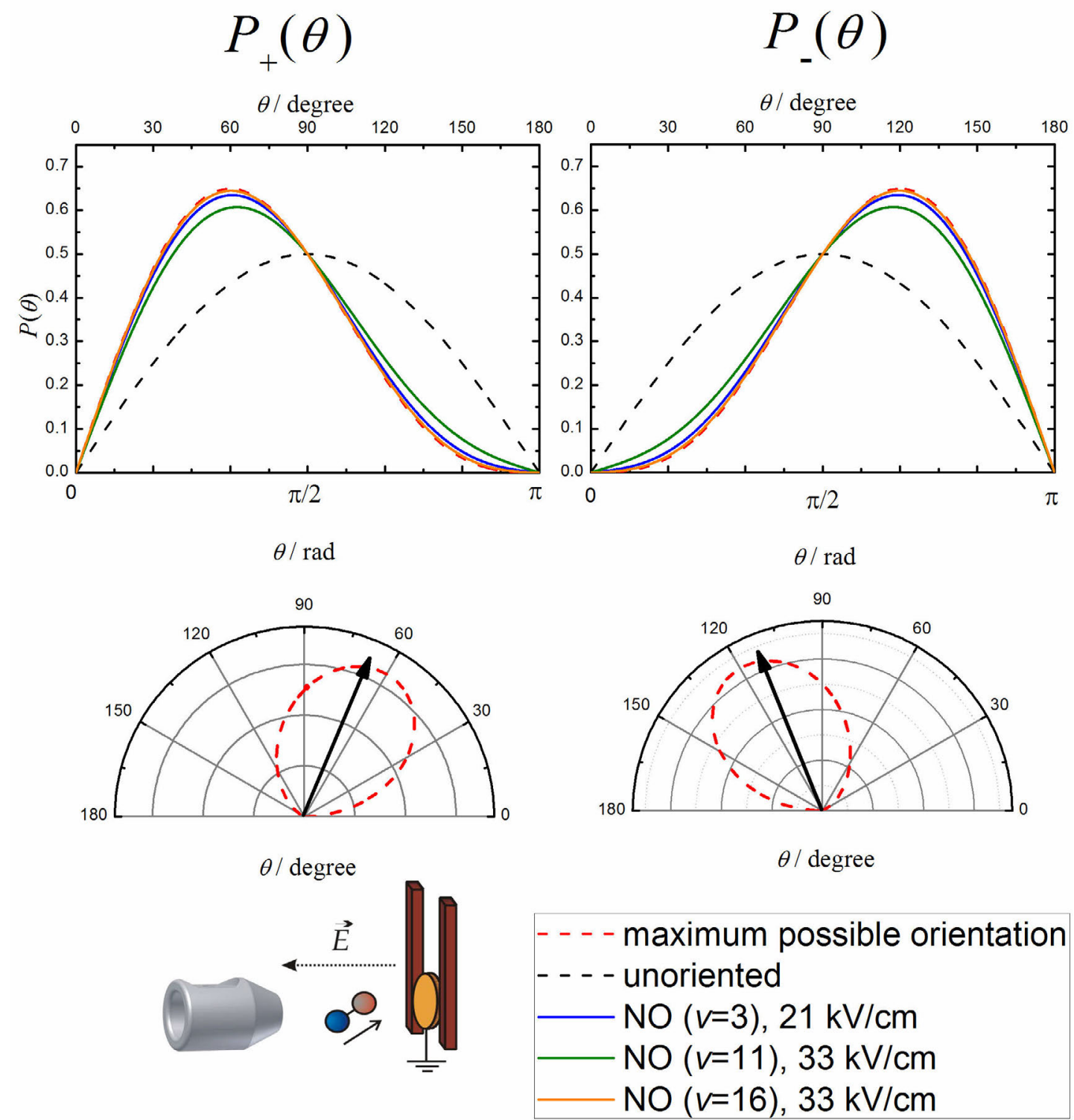

Figure 4.8: Orientation distributions for the NO scattering experiments - Top panels: Calculated orientation distribution functions $\left(P_{+}(\theta)\right.$ in the left panel and $P_{-}(\theta)$ in the right panel) for the different vibrational states of $\mathrm{NO} \mathrm{X}^{2} \Pi_{1 / 2}(v, J=|\Omega|=|M|=0.5)$ in $v=3,11$, and 16 are shown as blue, green and orange solid lines, respectively. The electric field strengths for these calculations are those used in the scattering experiments (chapter 6) and are given in the figure legend as well as in Table 4.1. The red and black dashed lines show orientation distributions in the limit of a strong electric field (maximum orientation) and field free (unoriented molecules), respectively. Bottom panels: Orientation distributions in the high-field limit (again $P_{+}(\theta)$ in the left panel and $P_{-}(\theta)$ in the right panel) are given as polar plots. The black arrows indicate the expectation values $\langle\theta\rangle_{\max }$ given by $67.5^{\circ}$ and $112.5^{\circ}$ for the two orientations, repectively. 
4. Orienting polar molecules without hexapoles 


\section{Suppression of spontaneous emission in the optical pumping of molecules: Pump-dump-sweep}

Optical pumping with laser radiation is an important tool for studying properties of excited electronic or vibrational states.(115, 116) In particular, highly excited vibrational states are typically prepared by stimulated emission pumping (SEP). SEP is a two-step optical pumping method via an excited electronic state to produce highly vibrationally excited molecules in the electronic ground state (see panel A of Fig. 5.1 for an example).

When we started to study the dynamics of highly vibrationally excited NO scattering from $\mathrm{Au}(111)$, we were however confronted with a major drawback of this incoherent method: the competition of stimulated and spontaneous emission leads to the population of not only the desired, but also a host of untargeted vibrational states. We thus developed a novel approach that improves the quantum state purity in the optical pumping by suppressing spontaneous emission. Subsequent to optical pumping similar to SEP, we depopulate the excited electronic state with a third laser pulse (the sweep step) that resonantly excites to rapidly dissociating states. We call this method pumpdump-sweep.

In this chapter, I will introduce the new method and demonstrate its successful ap- 


\section{Suppression of spontaneous emission in the optical pumping of molecules: Pump-dump-sweep}

plication for preparing $\mathrm{NO}$ molecules in $\mathrm{X}^{2} \Pi_{1 / 2}(v=16)$ by optical pumping via the $\mathrm{A}^{2} \Sigma^{+}(v=2)$ state. Here, the sweep step can reduce spontaneous emission from $\mathrm{A}^{2} \Sigma^{+}$ state by more than $90 \%$, thereby vastly improving the achieved quantum state purity. Furthermore, I will demonstrate the advantageous effect of this improved quantum state purity on a scattering experiment with the $\mathrm{Au}(111)$ surface. In the last section, I will compare our pump-dump-sweep results to those from an alternative approach for improving quantum state purity, namely stimulated Raman adiabatic passage (STIRAP).

\subsection{Introduction and description of the pump-dump-sweep concept}

Stimulated emission pumping (SEP) is a very powerful technique that allows access to highly vibrationally excited states in the electronic ground state of molecules.(117, 118) These states can hardly be populated by direct optical pumping via overtone excitation, as oscillator strengths fall rapidly with increasing vibrational target state. While SEP was first used in the field of molecular spectroscopy in molecular beams 1 (see (117) and references therein), it has also been applied to study resonant $v-$ $v$ energy transfer(119), the role of highly vibrationally excited $\mathrm{O}_{2}$ in stratospheric ozone formation(120, 121, 122), or the infrared lifetimes of highly vibrationally excited molecules(105). The electron emission experiments for highly vibrationally excited NO scattering from a low-workfunction Au surface $(57,59,60)$ as well as the Huang experiment (1) also used SEP.

Fig. 5.1 A shows how population in the 16 th excited vibrational state of $\mathrm{NO} \mathrm{X}^{2} \Pi_{1 / 2}(v=$ 16) can be produced via SEP. In a first step, molecules are excited via a rotationally resolved line of the $\mathrm{A}^{2} \Sigma^{+}\left(v^{\prime}=2\right) \leftarrow \mathrm{X}^{2} \Pi_{1 / 2}\left(v^{\prime \prime}=0\right)$ band (pump step) and are subsequently de-excited via the $\mathrm{A}^{2} \Sigma^{+}\left(v^{\prime}=2\right) \rightarrow \mathrm{X}^{2} \Pi_{1 / 2}\left(v^{\prime \prime}=16\right)$ band (dump step). The general problem with this technique is that - due to the competition of stimulated absorption and emission processes - population remains in the upper $\mathrm{A}^{2} \Sigma^{+}(v=2)$ state after the Pump and Dump laser pulses have been fired. This is a fundamental

\footnotetext{
${ }^{1}$ Among others, supersonic molecular beams offer the advantages, that the cooling of the rotation upon expansion allows for a high population in a starting level, that collisions are reduced, that the molecule density is high, and that the directed molecular beam can be excited under conditions with minimum Doppler broadening. This is very advantageous for optical pumping experiments.
} 
problem of incoherent optical pumping, as population remaining in the upper state is then transferred to untargeted vibrational states of the electronic ground state via spontaneous emission following the Franck-Condon factors of the $\mathrm{X}^{2} \Pi\left(v^{\prime \prime}\right) \leftarrow \mathrm{A}^{2} \Sigma^{+}\left(v^{\prime}=2\right)$ system.

To overcome this issue, we developed a new technique called pump-dump-sweep (Fig. 5.1B).

A) SEP

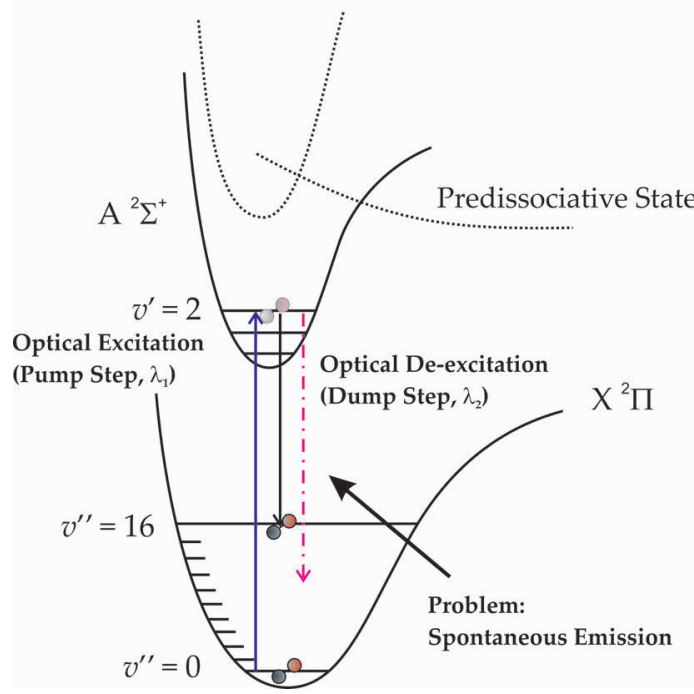

B)
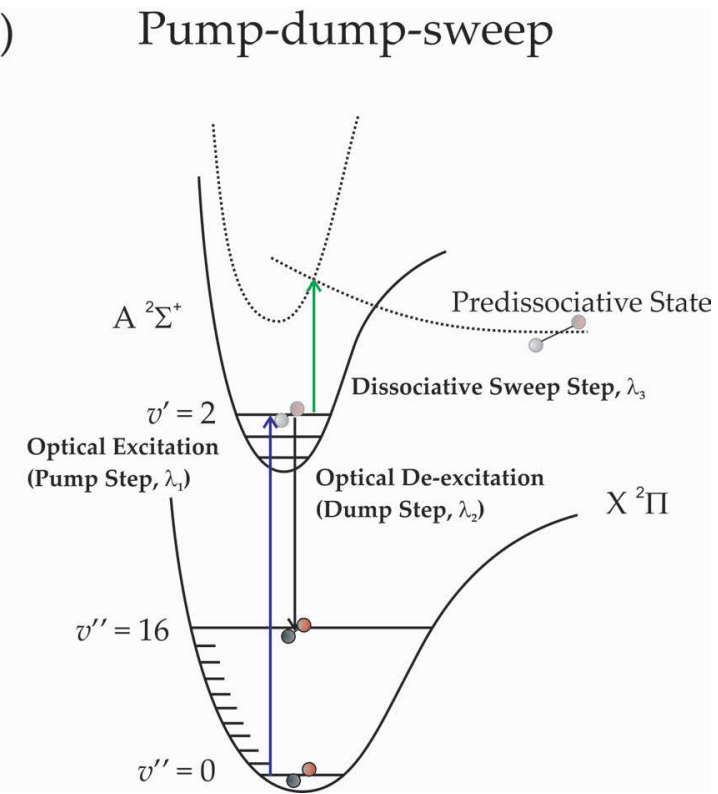

Figure 5.1: Stimulated emission pumping and pump-dump-sweep on the example of the NO molecule - (A) Stimulated Emission pumping (SEP) in the NO molecules can be performed via excitation to the $\mathrm{A}^{2} \Sigma^{+}\left(v^{\prime}=2\right)$-state (pump step) deexcitation to an excited vibrational state of the electronic ground state, e.g. to $\mathrm{X}^{1} \Sigma^{+}\left(v^{\prime \prime}=16\right)$ (dump step). However, after the laser pulses have been fired, population remaining in the upper state (which has a lifetime of approx. $200 \mathrm{~ns}$ ) is transferred to untargeted vibrational states. (B) In pump-dump-sweep, the fluorescence is suppressed by state-selective photodissciation (sweep step) with a laser that is fired after the pump and dump pulses. This vastly improves the quantum-state purity.

Since the population transfer from the $\mathrm{A}^{2} \Sigma^{+}\left(v^{\prime}=2\right)$ by fluorescence is rather slow (the $\mathrm{A}^{2} \Sigma^{+}\left(v^{\prime}=2\right)$ state has a lifetime of $\left.200 \mathrm{~ns}\right)$ compared to the optical pumping (approx. $6 \mathrm{~ns}$ pulse width for all lasers), there is a time window to suppress fluorescence by depopulating the $\mathrm{A}^{2} \Sigma^{+}\left(v^{\prime}=2\right)$ state after the pump and dump steps. This can be achieved with a third laser pulse that transfers population out of $\mathrm{A}^{2} \Sigma^{+}\left(v^{\prime}=2\right)$ to a higher electronic state that rapidly dissociates. The dissociation prevents competition 


\section{Suppression of spontaneous emission in the optical pumping of molecules: Pump-dump-sweep}

of absorption and stimulated emission in this transition and population in $\mathrm{A}^{2} \Sigma^{+}\left(v^{\prime}=2\right)$ can be removed efficiently.

\subsection{Implementation of the method and quantifying quan- tum state purity}

The first step to implement the pump-dump-sweep method was to find a suitable sweep transition. Therefore, a molecular beam of rotationally cold $\left(T_{\text {rot }} \approx 5 \mathrm{~K}\right) \mathrm{NO}$ molecules was produced in a supersonic jet expansion of $5 \% \mathrm{NO}$ seeded in $\mathrm{H}_{2}\left(E_{\mathrm{trans}}^{\mathrm{i}}=0.68 \mathrm{eV}\right)$ and molecules were electronically excited (pump step) in the differential chamber via the $\mathrm{A}^{2} \Sigma^{+}\left(v^{\prime}=2\right) \leftarrow \mathrm{X}^{2} \Pi_{1 / 2}\left(v^{\prime \prime}=0\right) \mathrm{Q}_{11}(0.5)$ transition at $204.708 \mathrm{~nm}\left(\lambda_{1}\right)$ using a home-built OPO laser systems with a pulse energy of $0.3 \mathrm{~mJ}$. The fluorescence of the $\mathrm{A}^{2} \Sigma^{+}\left(v^{\prime}=2\right)$ state was monitored with the photo multiplier tube and a dye laser $(8 \mathrm{~mJ} /$ pulse) was used to perform fluorescence depletion spectroscopy. Fig. 5.2 shows the fluorescence depletion spectrum for a wavelength range betwen 445 and $455 \mathrm{~nm}$. Within this wavelength range, we found five transitions with fluorescence depletions greater than $50 \%$. We decided to use the line with the strongest fluorescence depletion at $450.87 \mathrm{~nm}$ as a sweep transition. This transition was later tested to be saturated with pulse energies as small as $300 \mu \mathrm{J}$ at $5 \mathrm{~mm}$ beam diameter. This transition has not been assigned but is likely to excite to a rapidly dissociating state of one of the many electronically excited doublet states of NO above $6.5 \mathrm{eV}(123)$.

Fig. 5.3 shows the effect of the pump-dump-sweep approach in more detail. The left main panel shows the spontaneous emission transient observed on a PMT induced by the pump laser pulse. The characteristic decay time $(\tau=200 \mathrm{~ns})$ reflects the lifetime of the $\mathrm{A}^{2} \Sigma^{+}\left(v^{\prime}=2\right)$ state (124, 125, 126, 127). For the middle panel, a dump laser pulse dumping the $\mathrm{A}^{2} \Sigma^{+}\left(v^{\prime}=2\right) \rightarrow \mathrm{X}^{2} \Pi_{1 / 2}\left(v^{\prime \prime}=16\right) \mathrm{Q}_{11}(0.5)$ transition at $450.503 \mathrm{~nm}$ with the second home-built $\mathrm{OPO}$ with an energy of $0.5 \mathrm{~mJ} /$ pulse. The dump pulse was overlapped in space and time with the pump laser and leads to a depletion of the spontaneous emission by $20 \%$. This is a typical value for SEP (the maximum depletion that can be theoretically achieved by incoherent optical pumping is $33 \%$ for a coupled three-level system). In contrast to that, the sweep laser (right panel) resonant with the $450.87 \mathrm{~nm}$ sweep transition, fired $13 \mathrm{~ns}$ after the pump and dump pulses, suppresses spontaneous emission by more than $90 \%$. 


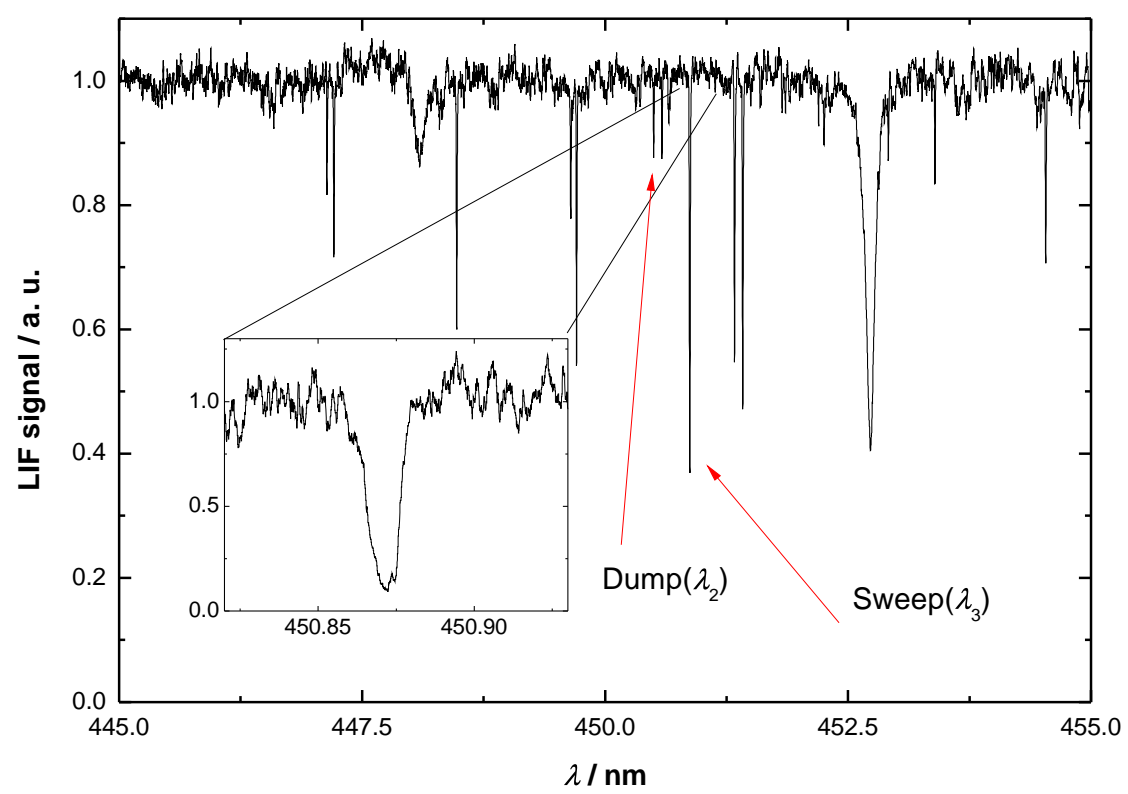

Figure 5.2: Scan of the sweep laser to find a sweep transition with strong fluorescence depletion - The graph shows the integrated laser induced fluorescene from the $\mathrm{A}^{2} \Sigma^{+}\left(v^{\prime}=2\right)$ state. The sweep laser which is overlapped with the pump laser in time and space is scanned and resonances with other states lead to a depletion of the fluorescence signal. The peak marked as "D" is the transition used as a DUMP step in SEP to prepare molecules in $\mathrm{X}^{2} \Pi_{1 / 2}\left(v^{\prime \prime}=16, J=0.5\right)$. The line labeled with $S$ is the chosen sweep transition. The inset shows an enlargement of the wavelength range around the sweep transition, scanned at lower scanning speed and with optimized spacial overlap between the two lasers. A depletion greater than $90 \%$ is observed. 
The insets of Fig. 5.3 show the population distributions produced in these three ex-
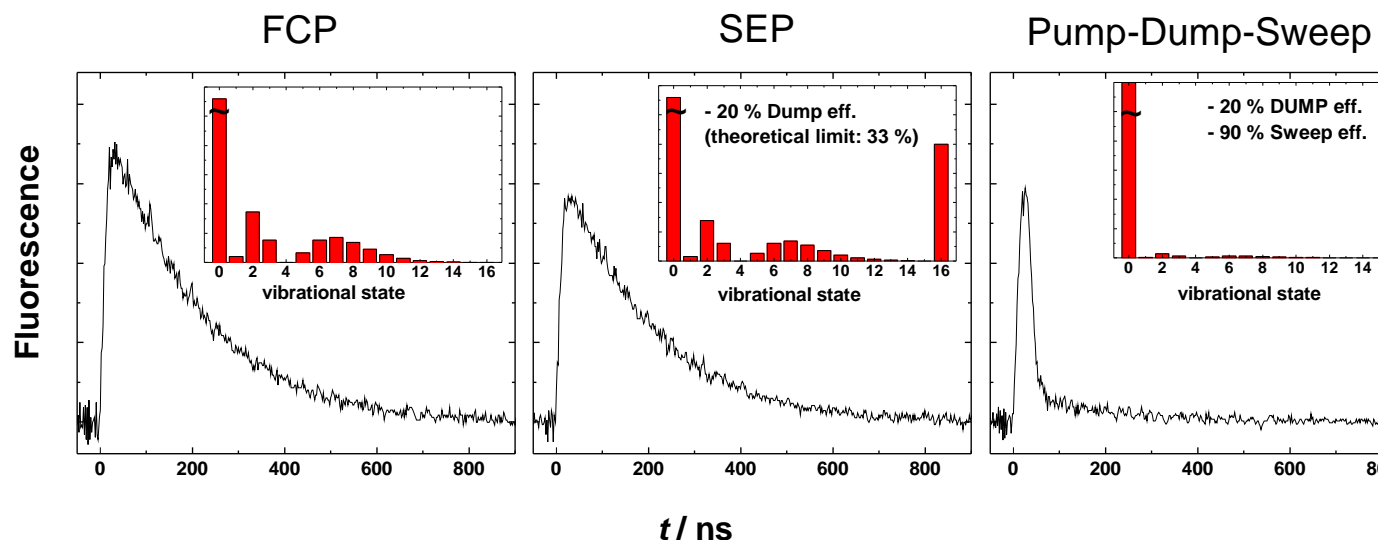

Figure 5.3: Fluorescence of the $\mathrm{A}^{2} \Sigma^{+}(v=2)$ state observed with a photomultiplier tube - (Left) Spontaneous emission from $\mathrm{A}^{2} \Sigma^{+}(v=2)$ induced by the pump laser (Franck-Condon pumping). (Middle) Fluorescence transient observed in a conventional SEP experiment, in which pump and dump lasers are simultaneously fired. The observed fluorescence depletion is $20 \%$. The theoretical maximum depletion is $33 \%$. (Right) Fluorescence transient observed in a pump-dump-sweep configuration. The sweep laser removes population from $\mathrm{A}^{2} \Sigma^{+}(v=2)$ after the population transfer from the pump and dump laser has taken place. The insets show the calculated population distributions in each case. The use of the sweep laser gives rise to a great improvement in quantum state purity. Adapted with permission from (128). Copyright 2013 American Chemical Society.

periments as calculated from Franck-Condon factors and the fluorescence traces. The interested reader can find the calculation in the Bachelor thesis of Sven Meyer(129). For conventional SEP (middle panel), the summed population of molecules in $v=6-13$ (produced by spontaneous emission) is comparable to the population of $v=16$, mostly produced by stimulated emission. In contrast to that, the sweep pulse (right panel) leads to a dramatic improvement in quantum state purity.

\subsection{Effect of the improved quantum state purity on molecule- surface scattering experiments}

In order to further demonstrate the effect of the improved quantum-state purity, we scattered NO molecules prepared in $\mathrm{X}^{2} \Pi_{1 / 2}(v=16, J=0.5)$ from a clean $\mathrm{Au}(111)$ surface at normal incidence. The collision populates a host of different vibrational 


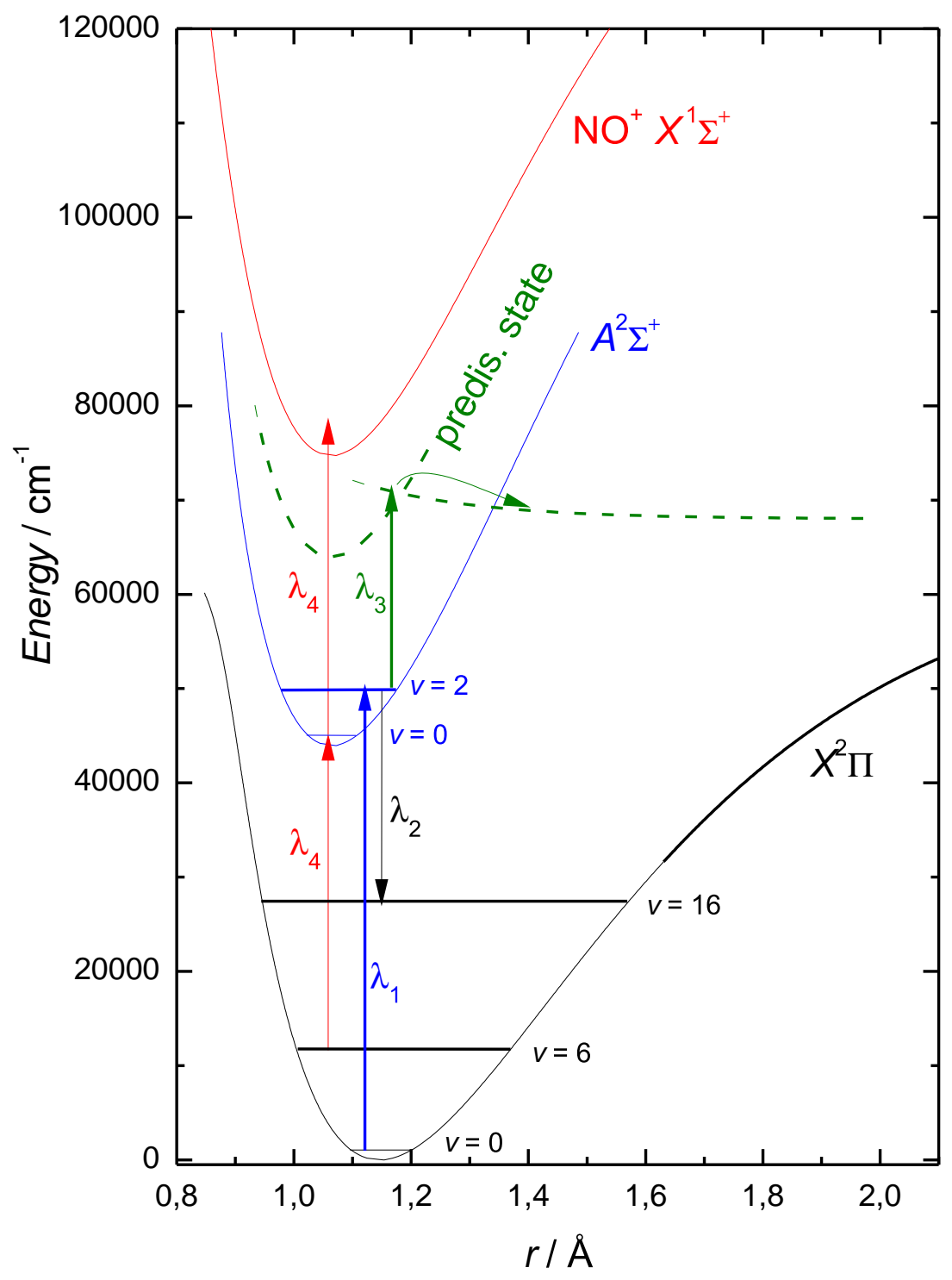

Figure 5.4: Energy diagram for the pump-dump-sweep-probe experiment in NO - Similar to Fig. $5.1 \mathrm{~B}$ the figure shows the optical preparation of $\mathrm{NO} \mathrm{X}^{2} \Pi^{+}\left(v^{\prime \prime}=16\right)$ by optical pumping through the $\mathrm{A}^{2} \Sigma^{+}(v=2)$ state followed by photo-dissociation of $\mathrm{A}^{2} \Sigma^{+}(v=2)$ (sweep step) to suppress fluorescence. The collision of $\mathrm{NO}(v=16)$ with a crystalline $\mathrm{Au}(111)$ surface populates a host of different vibrational states due to energy transfer between the molecule and the surface. In the experiment, we probe population in $\mathrm{X}^{2} \Pi(v=6)$ by resonantly ionizing via the $\mathrm{A}^{2} \Sigma^{+}(v=0)$ state (red arrows). The potentials (except for the still unassigned predissociative state) are calculated using NO molecular constants and the Rydberg-Klein-Rees method (RKR-method) for determining potential energy curves of diatomic molecules. Adapted with permission from (128). Copyright 2013 American Chemical Society. 


\section{Suppression of spontaneous emission in the optical pumping of molecules: Pump-dump-sweep}

states due to energy transfer between the molecule and the surface. Scattered molecules were then probed with REMPI spectroscopy in a wavelength region mostly probing population in the vibrational state $\mathrm{X}^{2} \Pi(v=6)$ by ionization via the $\mathrm{A}^{2} \Sigma^{+}(v=0)$ state, see Fig. 5.4 .

Fig. 5.5 (left column) shows measured REMPI spectra, where molecules are prepared via SEP (without using the sweep pulse). Naively, one would expect the signal to reflect the probability for the relaxation channel $\mathrm{NO} \mathrm{X}^{2} \Pi(v=16 \rightarrow v=6$ ) (upper left panel). However, the signal does not vanish when the dump laser (which creates population in $\left.\mathrm{X}^{2} \Pi(v=16)\right)$ is blocked (lower left panel). This is because the probed vibrational state $v=6$ is not only populated by the scattering channel $\mathrm{X}^{2} \Pi(v=16 \rightarrow 6)$ but also by scattering channels originating from untargeted vibrational states produced by SEP such as $\mathrm{X}^{2} \Pi(v=6 \rightarrow 6, v=7 \rightarrow 6, v=8 \rightarrow 6)$, and so forth. This means, that the scattering experiment is ruined by spontaneous emission.

As can be seen from the right panels of Fig. 5.5, the impact of the sweep laser is dramatic. In this case, the desired pump-dump-sweep-probe signal is much stronger than the background produced by spontaneous emission. This reveals the collision induced $\mathrm{X}^{2} \Pi(v=16 \rightarrow 6)$ channel. Only this tremendous amount of improvement of signal to background allows for answering detailed questions about the collision process such as the influence of the incidence molecular orientation, translational energy or the rotational state distributions of surface scattered molecules (see chapter 6).

\subsection{Comparison of the pump-dump-sweep approach to stimulated Raman adiabatic passage (STIRAP)}

Pump-dump-sweep is not the first approach to suppress spontaneous emission in optical pumping. An alternative method is stimulated Raman adiabatic passage (STIRAP), see reviews $(130,131,132)$ and references therein. STIRAP is a method based on coherent optical pumping, which allows for a complete population transfer from an initial state $|1\rangle$ to a final state $|3\rangle$, see Fig. 5.6. STIRAP uses a counter-intuitive pulse sequence in which a Stokes pulse coupling the levels $|2\rangle$ and $|3\rangle$ with the Rabi-frequency $\Omega_{S}$ precedes the the pump pulse coupling the levels $|1\rangle$ and $|2\rangle$ with the Rabi-frequency 


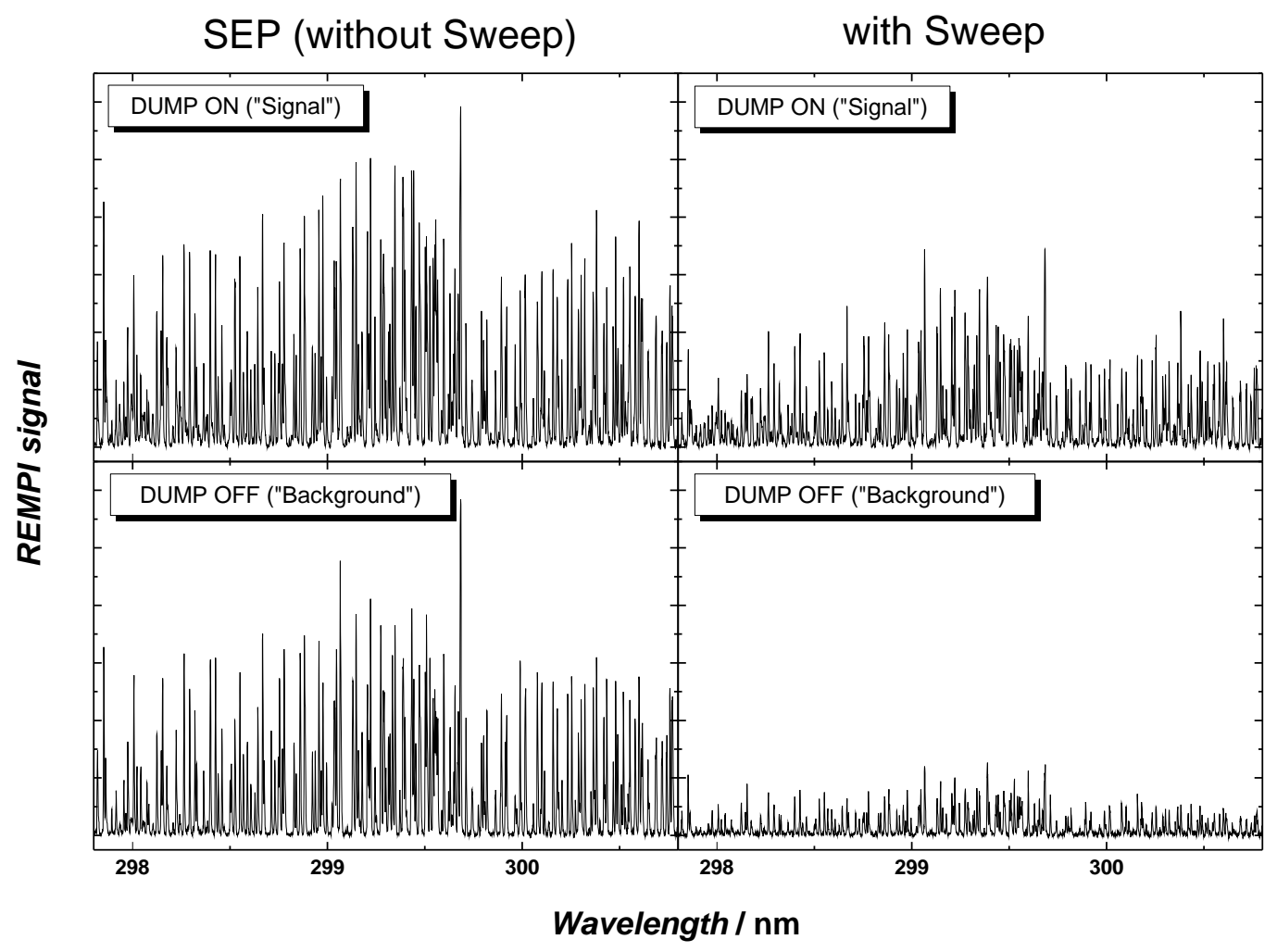

Figure 5.5: Application of the pump-dump-sweep approach to a gas-surface scattering experiment - The figure shows results from a pump-dump-probe experiment where $\mathrm{NO} \mathrm{X}^{2} \Pi_{1 / 2}(v=16, J=0.5)$ is produced by SEP. The molecular beam then collides with the (111) surface of a clean gold crystal. The probe laser (which is scanned in the spectra shown) detects population in NO $\left(\mathrm{X}^{2} \Pi(v=6)\right.$ with $(1+1)-\mathrm{REMPI}$ via the $A^{2} \Sigma^{+}(v=2)$ state. The many lines result from the rotational resolution in the experiment. For the two panels on the left side, the sweep pulse is not used. In this case, the effect of the dump laser (dumping to $\mathrm{X}^{2} \Pi(v=16)$ is difficult to discern. This is because the REMPI signal is dominated by population from molecules that were not dumped to $v=16$, but instead were produced in other vibrational states due to spontaneous emission and ended up in $v=6$ after the collision with the surface. The right panels demonstrate the effect of the sweep laser on the scattering experiment. In this case, spontaneous emission is efiiciently suppressed and the influence of the dump laser is clearly seen. This way, the specific scattering channel $v=16 \rightarrow v=6$ is revealed. Adapted with permission from (128). Copyright 2013 American Chemical Society. 

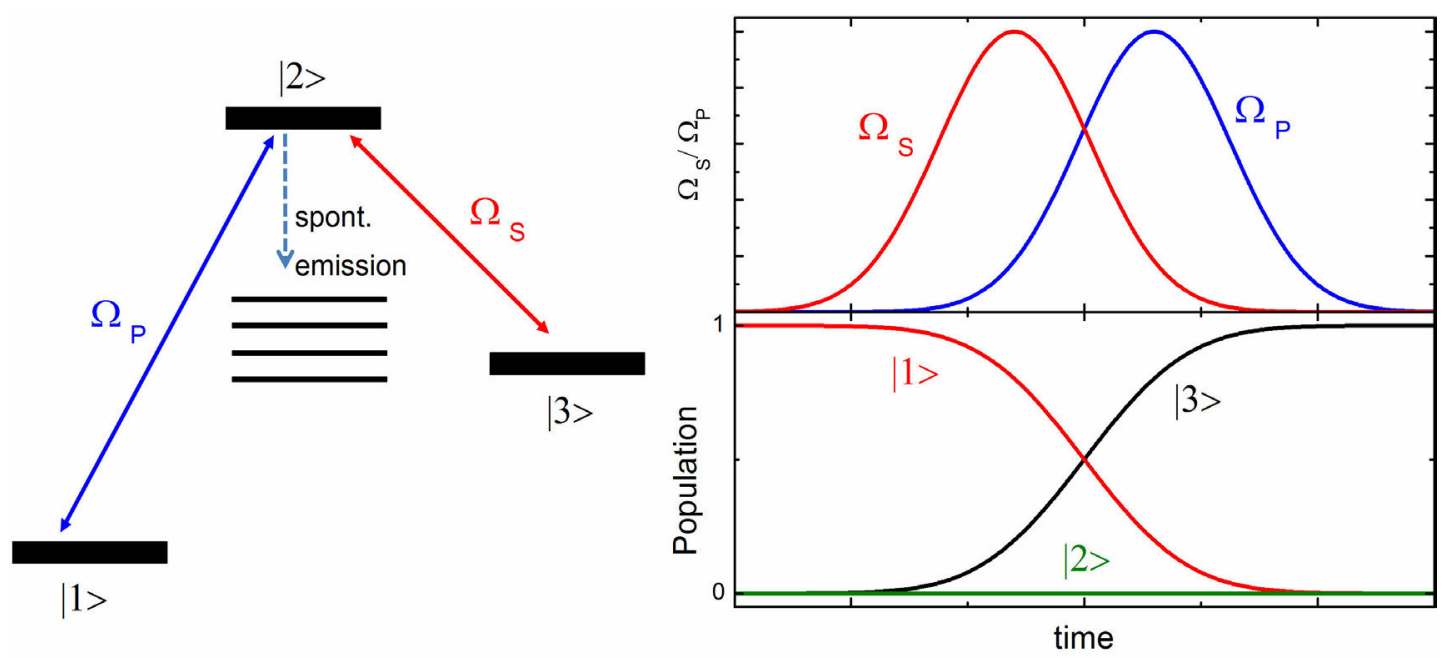

Figure 5.6: Concept of stimulated Raman adiabatic passage (STIRAP) - (left) Schematic of a three-level system coupled by the pump pulse with Rabi-frequency $\Omega_{P}$ and the Stokes pulse with the Rabi-frequency $\Omega_{S}$. (right) As displayed in the top panel, the Stokes pulse preceedes the pump pulse in time and the pulses overlap only partially. The bottom panel shows the time evolution of the states $|1\rangle,|2\rangle$ and $|3\rangle$. In the ideal case, an complete population transfer between the initially populated state $|1\rangle$ and the final state $|3\rangle$ is achieved. 


\subsection{Comparison of the pump-dump-sweep approach to stimulated Raman}

adiabatic passage (STIRAP)

$\Omega_{P}$. Solving the time-dependent Schrödinger equation for this coupled three-level system within the rotating-wave approximation yields, that the entire population from level $|1\rangle$ is transferred to level $|3\rangle$ at appropriate time delay. During this process, the intermediate level $|2\rangle$ is a dark state and never gets populated. Thus, no spontaneous emission to untargeted states can occur.

STIRAP requires highly coherent light sources and is thus most straight-forward using single-mode cw-lasers. Here, STIRAP has been successfully applied in many different fields such as chemical reaction dynamics (133), ultra-cold atoms (134) or atom optics (135). Unfortunately, current high-power and high-resolution cw-laser technology is limited to wavelengths longer or within the visible region and only few molecules have low-lying electronic states, which make them suitable candidates for STIRAP.

The alternative to STIRAP with $\mathrm{cw}^{-}$-lasers is to use pulsed lasers, which are available in the ultra-violet but have inferior coherence properties (136, 137). Indeed, pulsed STIRAP has been used previously to prepare NO molecules in $\mathrm{X}^{2} \Pi_{1 / 2}(v=$ $6, J=0.5)$ (138, 139). Following this work, we tested pulsed STIRAP to prepare molecules in $\mathrm{NO} \mathrm{X}^{2} \Pi_{1 / 2}(v=11, J=0.5)$ using our home-built OPO laser systems for the pump and Stokes transitions. The interested reader will find details on this experiment in the masters thesis from Bastian Krüger.(140) Briefly, we pumped the $\mathrm{A}^{2} \Sigma^{+}\left(v^{\prime}=2\right) \leftarrow \mathrm{X}^{2} \Pi_{1 / 2}\left(v^{\prime \prime}=0\right) \mathrm{Q}_{11}(0.5)$ transition at $204.708 \mathrm{~nm}(0.5 \mathrm{~mJ} / \mathrm{pulse}$, $1 \mathrm{~mm}$ beam diameter) with one OPO and used the other OPO as a Stokes laser for the $\mathrm{A}^{2} \Sigma^{+}\left(v^{\prime}=2\right) \rightarrow \mathrm{X}^{2} \Pi_{1 / 2}\left(v^{\prime \prime}=11\right) \mathrm{Q}_{11}(0.5)$ transition at $336.101 \mathrm{~nm}(0.5 \mathrm{~mJ} / \mathrm{pulse}$, $2 \mathrm{~mm}$ beam diameter). Molecules were prepared in the differential chamber and population in the target state was probed $20 \mathrm{~cm}$ downstream in the surface chamber with REMPI spectroscopy. We then changed the delay between pump and Stokes lasers with a manual delay stage ( $3 \mathrm{~m}$ long) and detected the population in $v^{\prime \prime}=11$ with and without blocking the Stokes pulse, see Fig. 5.7.

Unfortunately, we did not manage to increase the transfer efficiency by firing the Stokes laser before the pump laser. Instead, the transfer efficiency behaved as expected for incoherent pumping.

Although we did not try STIRAP for a long time (we stopped testing it once we found that pump-dump-sweep works nicely for our scattering experiments), our unsuccessful first attempt shows, that pulsed STIRAP can be challenging to implement. A possible issue is that pulsed STIRAP requires highly coherent laser radiation, strong transitions 


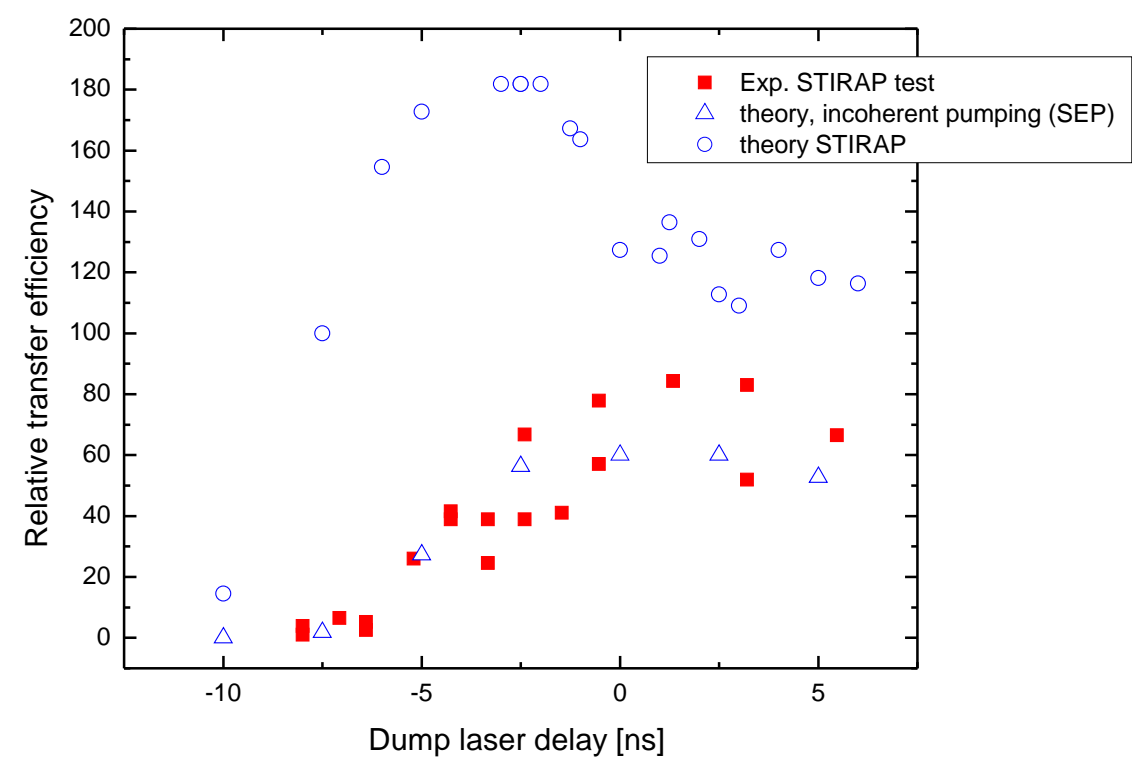

Figure 5.7: Unsuccessful attempt to implement STIRAP - The figure shows the relative transfer efficiency as function of the delay between pump and Stokes lasers. A negative delay means, that the Stokes laser preceeds the pump laser (both lasers have a pulse duration of approx. $6 \mathrm{~ns}$ ). The relative transfer efficiency is given on a scale that compares the REMPI signal detecting population in the targeted final state $|3\rangle$ (here NO $\left.\mathrm{X}^{2} \Pi_{1 / 2}(v=11, J=0.5)\right)$ produced with Stokes laser to the population produced without Stokes laser (Franck-Condon pumping). Experimental data from this work is given as red solid squares. For comparison the graph also shows arbitarily rescaled values calculated by Schiemann et al.(138) for incoherent optical pumping (calculated using rate equations, blue open triangles) and coherent pumping (which corresponds to STRIRAP in the delay range between -5 and $-2 \mathrm{~ns}$, blue open circles). It is found, that the attempt to implement STIRAP has not been successfull. 
for the optical pumping steps and high laser power to satisfy the criterion for adiabatic following. For pulsed lasers, this criterion is given by (138)

$$
\Omega_{\mathrm{eff}}^{2} \Delta \tau>100 \frac{1}{\Delta \tau}\left(1+R^{2}\right)
$$

where $\Delta \tau$ is the pulse length of the laser pulse $(\Delta \tau=6 \mathrm{~ns}$ for Nd:YAG pumped laser systems), $\Omega_{\mathrm{eff}}$ is the effective Rabi-frequency, and $R=\frac{\omega_{\mathrm{L}}}{\omega_{\mathrm{TL}}}$ is the ratio of the actual laser bandwidth $\left(\omega_{L}, 250 \mathrm{MHz}\right.$ for our home-built OPOs) to the transformlimited bandwidth $\left(\omega_{\mathrm{TL}}\right)$. The transform-limited bandwidth is for idealized Gaussian shaped pulses given by $\omega_{T L} \approx 0.44 / \Delta \tau$ and gives a value of $73 \mathrm{MHz}$ for $\Delta \tau=6 \mathrm{~ns}$. The effective Rabi-frequency $\Omega_{\text {eff }}$ can be calculated from the Rabi-frequencies for the pump and Stokes transitions according to $\Omega_{\mathrm{eff}}=\sqrt{\Omega_{\mathrm{P}}^{2}+\Omega_{\mathrm{S}}^{2}}$. The Rabi-frequency for on-resonance excitation is given by

$$
\Omega=\frac{\mu_{12} \cdot E}{\hbar},
$$

where $\mu_{12}$ is the transition dipole moment between two states (it can be calculated from the Einstein coefficients (141) ) and $E$ is the electric field amplitude created by the laser radiation. The electric field amplitude is connected to the irradiance $I$ of the laser pulse via the speed of light $c$ and the vacuum permittivity $\epsilon_{0}$ (141)

$$
I=\frac{1}{2} c \epsilon_{0} E^{2}
$$

The irradiance is time dependent, but an average value can be calculated from the pulse energy $E_{\text {pulse }}$, the pulse length $\Delta \tau$ and the area $A$ of the laser pulse $I=\frac{E_{\text {pulse }}}{\Delta \tau A}$. For our STIRAP test, I calculated the left side of equation 5.1 to $\Omega_{\text {eff }}^{2} \Delta \tau=4.87 \cdot 10^{12} \mathrm{~Hz}$ and the right side to $100 \frac{1}{\Delta \tau}\left(1+R^{2}\right)=2.09 \cdot 10^{11} \mathrm{~Hz}$. This means, that the adiabatic criterion should in principle have been fulfilled in the experiment. Up to this point, it is thus not clear to me, why we did not observe any signature of a STIRAP process. As already mentioned, Schiemann et al. (138) managed to implement STIRAP for the preparation of $\mathrm{NO} \mathrm{X}^{2} \Pi_{1 / 2}(v=6, J=0.5)$. The authors reported that the Stokes laser induced a fluorescence depletion from the intermediate state $|2\rangle$ of $40 \%$ in their experiment. The finding that the fluorescence was not depleted by $100 \%$ was attributed to "fluorescence from the spatial wings of the pump laser, where the maximum Rabi frequency is too low for the STIRAP process but high enough to populate level $|2\rangle$ ” 
(138). Compared to this, the fluorescence depletion of $90 \%$ we observed with the pumpdump-sweep approach is far superior.

This means, that pump-dump-sweep (PDS) can for some experiments be an interesting alternative to STIRAP. STIRAP has the the advantages that 1) it in principle (meaning for $\mathrm{cw}$-lasers) allows for a full population transfer, and that 2) it uses two laser systems instead of the three laser required for PDS. PDS can be an attractive alternative for optical pumping in particular in the UV (where cw-lasers are not available) and has the advantage, that it is based on incoherent optical pumping and is thus less challenging to implement. The generality of the PDS approach depends on the question of whether appropriate strong sweep transitions for photo-dissociation can be found. In case no such states are available, it could also be possible to use alternative approaches for a state-selective extinguishing of population in the intermediate state (e.g. by resonant ionization) or state-selective deflection (e.g. by electric or magnetic fields). 
6

\section{Vibrationally inelastic scattering of oriented NO from $\mathrm{Au}(111)$}

This chapter contains results and discussion of scattering experiments for vibrationally excited NO colliding with a $\mathrm{Au}(111)$ surface. I will first (section 6.1) show results for NO initially prepared in $v_{\mathrm{i}}=3$ by means of vibrational overtone pumping and then (section 6.2 show the results for the scattering of NO initially prepared in high vibrational states $\left(v_{\mathrm{i}}=11\right.$ and 16$)$. The vibrational relaxation depends strongly on the molecules orientation, the incidence vibrational state and incidence translational energy. Furthermore, very interesting effects regarding the rotational state distributions - namely the observation of strong rotational rainbows, and the effect of rotational cooling upon vibrational relaxation - are observed. I will explain the origin of these effects according to my current understanding and also compare the results to recent $a b$ intio calculations in the discussion (section 6.3). We already published some of the data and ideas presented in this chapter $(4,6,6,6$, 7).

\subsection{Vibrationally inelastic scattering of NO $(v=3)$ from $\mathrm{Au}(111)$}

\subsubsection{Experimental}

For the molecular beam scattering experiments described in this section, we generated pulsed supersonic molecular beams with different translational energies by expanding 
mixtures of NO in different carrier gases (Table 6.1) into vacuum. The table also contains data for the gas mixtures used in the experiments with high vibrational states which will be described in Section 6.2.

Table 6.1: Incidence translational energies $E_{\text {trans }}$ of the different NO/carrier gas mixtures used for molecular beam surface scattering experiments with NO prepared in $v_{\mathrm{i}}=3,11$ and 16 . The translational energies slightly vary for the formally similar gas mixtures due to uncertainties in the preparation. The molecular beams have a spread of the translational energy of $\approx 15 \%$ (FWHM).

\begin{tabular}{cccc}
\hline \hline Mixture & $E_{\text {trans }}^{\mathrm{i}}\left(v_{\mathrm{i}}=3\right) / \mathrm{eV}$ & $E_{\text {trans }}^{\mathrm{i}}\left(v_{\mathrm{i}}=11\right) / \mathrm{eV}$ & $E_{\text {trans }}^{\mathrm{i}}\left(v_{\mathrm{i}}=16\right) / \mathrm{eV}$ \\
\hline $1 \% \mathrm{NO}$ in $\mathrm{H}_{2}$ & - & 0.95 & 0.97 \\
$2 \% \mathrm{NO}$ in $\mathrm{H}_{2}$ & 0.89 & - & - \\
$5 \% \mathrm{NO}$ in $\mathrm{H}_{2}$ & 0.68 & 0.69 & 0.66 \\
$10 \% \mathrm{NO}$ in $\mathrm{H}_{2}$ & 0.48 & 0.51 & 0.52 \\
$25 \% \mathrm{NO}$ in $\mathrm{H}_{2}$ & 0.27 & - & 0.23 \\
$60 \% \mathrm{NO}$ in $\mathrm{H}_{2}$ & - & 0.14 & - \\
$60 \% \mathrm{NO}$ in $\mathrm{Ar}$ & 0.08 & - & - \\
$60 \% \mathrm{NO}$ in $\mathrm{Kr}$ & - & 0.05 & 0.05 \\
\hline \hline
\end{tabular}

Part of the NO population is transferred to $\mathrm{NO}(v=3)$ by means of vibrational overtone pumping with the IR-laser system (see section 3.3.1). The excellent resolution and power of the IR-laser system $(130 \mathrm{MHz}$ linewidth, $>17 \mathrm{~mJ} /$ pulse in the IR at around $5544 \mathrm{~cm}^{-1}$ were focussed on the molecular beam with a $\mathrm{f}=500 \mathrm{~mm}$ cylindrical lens) allows for exciting either the high field seeking $(e)$-parity or the low field seeking $(f)$-parity state in $\mathrm{NO} \mathrm{X}^{2} \Pi_{1 / 2}(v=3, J=0.5)$ via the $\mathrm{X}^{2} \Pi_{1 / 2}(v=3) \leftarrow \mathrm{X}^{2} \Pi_{1 / 2}(v=$ $0) Q_{11}(0.5)_{e / f}$ transition, see Fig. 6.1. Molecules then fly into the surface chamber and are oriented by the electric field generated by the high voltage electrode. Voltages of up to $\pm 15 \mathrm{kV}$ were applied to the electrode, generating electric fields up to $\pm 21.4 \mathrm{kV} / \mathrm{cm}$ at an electrode-surface distance of $7 \mathrm{~mm}$ (the electric field strength was verified by measuring the Stark splitting for a system with known molecular constants). The orientation distributions under these conditions have been calculated in section 4.5. The collision with the clean $\mathrm{Au}(111)$ surface at normal incidence and room temperature leads to energy transfer and many rotational levels of $v=3,2,1$, and 0 become populated. $1 \mu \mathrm{s}$ after the collision, the electric field is pulsed to ground with the high-voltage switch and the population in different ro-vibrational states is probed 


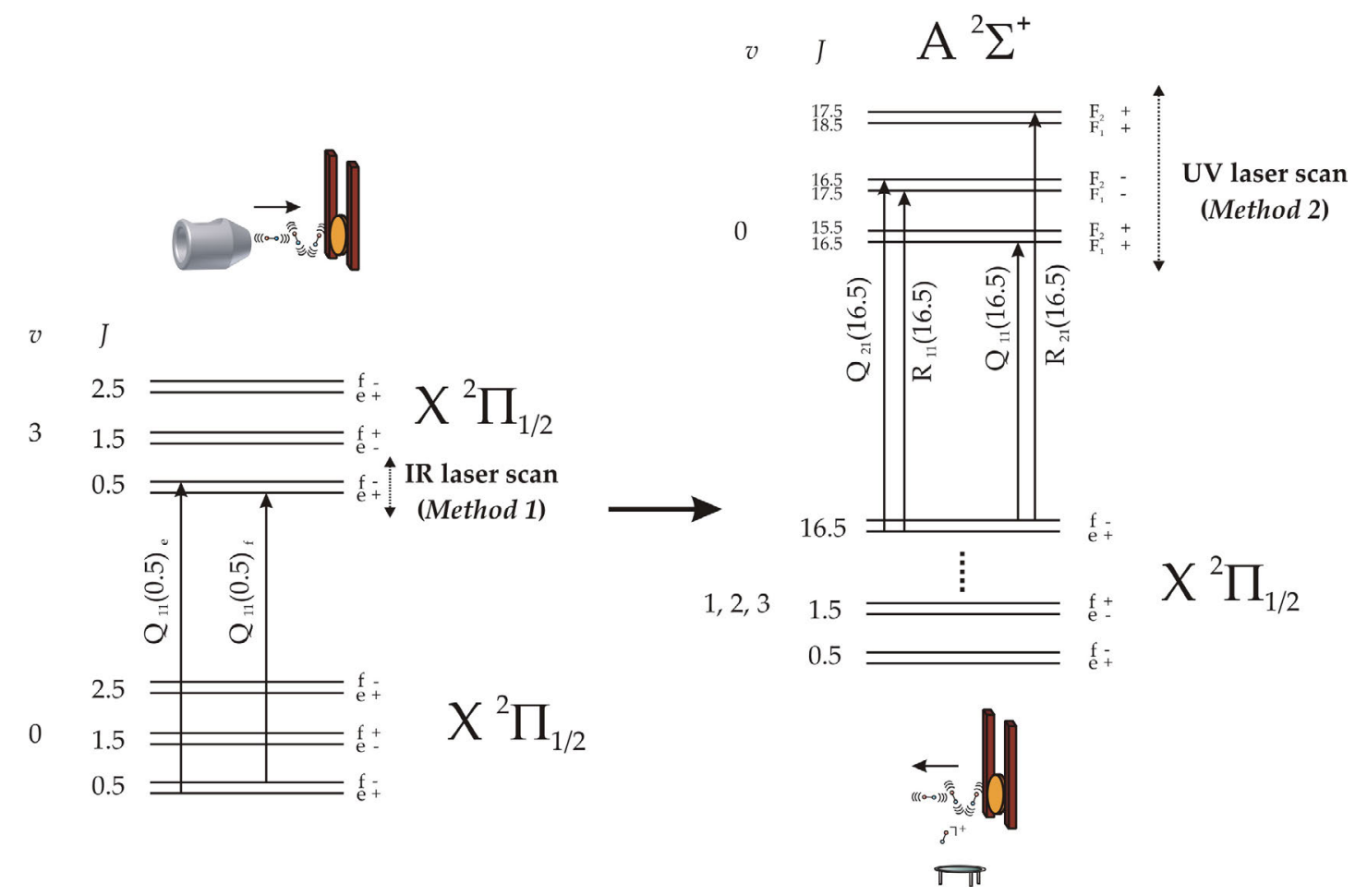

Figure 6.1: Pump-probe scheme of the performed experiment. - In a first step, the IR laser excites either the high field seeking state or the low field state in $v=3$ by vibrational overtone pumping via the $\mathrm{Q}_{11}(0.5)_{e}$ or $\mathrm{Q}_{11}(0.5)_{f}$ transition, respectively. By applying and electric field prior to the collision, these states evolve into oriented states before scattering from the $\mathrm{Au}(111)$ surface. After scattering, the electric field is pulsed to ground and the population of $\mathrm{NO}$ molecules in different rotational states of $v=3,2,1$ is probed by $(1+1)-$ REMPI spectroscopy via the $\mathrm{A}^{2} \Sigma^{+}(v=0)$-state. The dotted arrows illustrate the two different approaches towards demonstrating the influence of the molecular orientation. In method 1 , the pump laser is scanned over the $\Lambda$-doublet, while the probe laser is fixed, detecting a specific ro-vibrational state. Alternatively, in method 2, the pump laser is fixed at either the low or the high field seeking state (corresponding to the different orientations) and the probe laser is scanned. Reproduced with permission from (5). Copyright 2014 American Chemical Society. 
with REMPI spectroscopy via the $\mathrm{A}^{2} \Sigma^{+}(v=0)$ state (Fig. 6.1. right). For REMPI spectroscopy, molecules were resonantly ionized close to the surface with the second harmonic of the Sunlite system (2 mJ/pulse @ $255 \mathrm{~nm}$ ) with a large $4 \mathrm{~mm}$ beam diameter. This way, we detect an average of molecules scattered in all scattering angles (although the scattered angular distributions are narrow anyway (10)). Molecules relaxing to $v=0$ cannot be detected due to a large background in the REMPI signal from molecules not excited by the IR laser $(v=0 \rightarrow 0$ scattering channel) and also REMPI spectra probing population in NO $v=1$ have significant background due to the scattering channels $v=1 \rightarrow 1$ (as some population in $v=1$ is also generated in the supersonic jet expansion) and $v=0 \rightarrow 1$ (vibrational excitation).

\subsubsection{Probing the steric effect using method 1}

The direction in which the NO molecules point (N-first vs. O-first orientation) or more exactly the direction with higher probability as shown in the orientation distributions depends on the combination of 1$)$ the selected state in optical pumping $((e)$ vs. (f)-parity), 2) the direction of the dipole moment (given in Fig. 4.7 in the selected vibrational state and 3) the polarity of the voltage applied to the orientation electrode. NO $(v=3, J=0.5)$ prepared in the low-field seeking $(f)$-parity state via the $\mathrm{Q}_{11}(0.5, e)$ line at $5544.0039 \mathrm{~cm}^{-1}$ is orientated with the $\mathrm{O}$-atom pointing towards the surface, if the voltage on the electrode is positive. The orientation of incoming NO molecules can be switched by either changing the polarity of the electric field ("flipping via field polarity", as done in hexapole orientation experiments) or by pumping the other component of the $\Lambda$-doublet ("optical flipping") at $5544.0321 \mathrm{~cm}^{-1}$.

Fig. 6.2 shows an illustrative example of how the steric effect in a gas-surface collision can be probed. The left panels show the REMPI signal detected when the UV laser was fixed at a REMPI transition probing population in one particular ro-vibrational level of scattered NO. At the same time, the IR laser is scanned over the $\Lambda$-doublet to optically flip the molecular orientation. This corresponds to method 1 as depicted in Fig. 6.1. In the particular example, we probe population in the highly rotationally excited state $\mathrm{NO}(v=3, J=35.5)$ produced by collisions of $\mathrm{NO}(v=3, J=0.5)$ with an incidence translational energy of $0.89 \mathrm{eV}$. Without an electric field (left, center panel) the optical pumping of either of the components of the $\Lambda$-doublet generates the same isotropic orientation distributions and the magnitude of the REMPI signal 

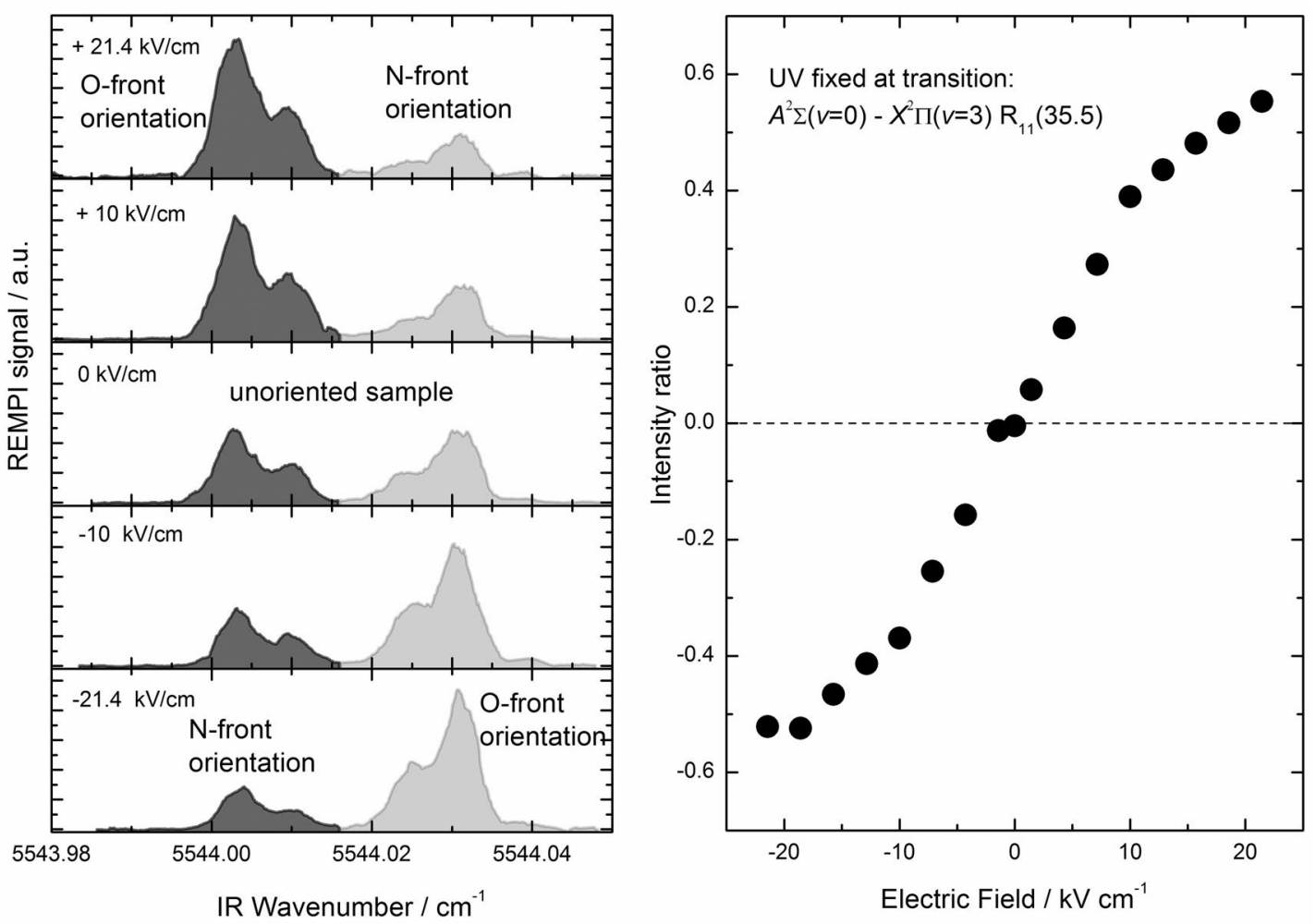

Figure 6.2: Demonstration of orientation dependent scattering: detecting a single, scattered ro-vibrational state - (left panels) REMPI signal detecting the population of $\mathrm{NO} \mathrm{X}^{2} \Pi_{1 / 2}(v=3, J=35.5)$ produced by the collision of $\mathrm{NO} \mathrm{X}^{2} \Pi_{1 / 2}(v=3, J=0.5)$ with the $\mathrm{Au}(111)$ surface (indicated as method 1 in Fig. 6.1 and in the text). The two features (marked as dark and light shaded areas, respectively) in the IR overtone spectrum ( $\Lambda$-doublets) correspond to optical pumping of opposite parity states, which evolve to states of different orientation after the molecule enters the electric field. The different panels are for different voltages applied to the orientation electrode. The high rotational state detected $(v=3, J=35.5)$ is more preferably populated by molecules incident with the $\mathrm{O}$-atom oriented towards the surface than for molecules incident with $\mathrm{N}$-atom towards the surface. Thus, pumping the lower wavelength component leads to more REMPI intensity at positive voltages and pumping the higher wavelength component at negative voltages (both combinations lead to an $\mathrm{O}$-first orientation). The substructure in each of the $\Lambda$-doublets results from partially resolved hyperfine splitting(142, 143, 144) due to the nuclear spin in the molecule. (right panel) Ratio of the integrated intensities of each of the $\Lambda$-doublet components as function of the electric field strength. At high electric field strength, the asymmetry approaches saturation, as the maximum possible degree of orientation is achieved. 


\section{Vibrationally inelastic scattering of oriented NO from $\mathrm{Au}(111)$}

is independent of which of the components is pumped. This changes dramatically in case oriented samples are produced by applying a voltage to the electrode. Here, we find that the REMPI signal is much higher if molecules are oriented with the $\mathrm{O}$-atom towards the surface. This reflects that the detected high rotational state is more likely populated by $\mathrm{O}$-first collisions than by $\mathrm{N}$-first collisions. The intensity ratio between both bands can be measured as function of the electric field strength. In agreement with the measurements determining the degree of orientation explained in section 4 the orientation and therewith the asymmetry in the detected REMPI signal approaches saturation at the highest electric fields $\pm 21.4 \mathrm{kV} / \mathrm{cm}$ (right panel of Fig. 6.2).

As the $\mathrm{NO} \mathrm{X}^{2} \Pi_{1 / 2}(v=3, J=35.5)$ level is more populated by $\mathrm{O}$-first collisions than by $\mathrm{N}$-first collisions, there necessarily must be other ro-vibrational states preferably populated by $\mathrm{N}$-first collisions. Fig. 6.3 shows that there are indeed ro-vibrational states (e.g. the $(v=2, J=15.5)$ shown in panel B) with opposite asymmetry in the observed spectra.

\subsubsection{REMPI spectra from oriented scattering using method 2}

For the results shown so far, the REMPI laser was set to a fixed transition and the IR pumping laser was tuned over the $\Lambda$-transition (method 1 of Fig. 6.1). More information can be obtained, if the IR laser is locked to excite to either of the different parity states and instead the UV laser is scanned over entire ro-vibrational bands (method 2 of the same figure). Fig. 6.4 shows resulting REMPI spectra for each orientation at five different incidence translational energies between $0.08 \mathrm{eV}$ and $0.89 \mathrm{eV}$. The IR laser is locked to a transition inducing either $\mathrm{N}$-first (left panels) or O-first (right panels) orientation at an electric field strength of $21.4 \mathrm{kV} / \mathrm{cm}$ (method 2). The UV laser scans over the $\mathrm{A}^{2} \Sigma^{+}(v=0) \leftarrow \mathrm{X}^{2} \Pi_{\Omega}(v=2)$ and $\mathrm{A}^{2} \Sigma^{+}(v=0) \leftarrow \mathrm{X}^{2} \Pi_{\Omega}(v=3)$ bands. The REMPI spectra have been normalized to the laser power according to an experimentally determined linear power dependence.

In the REMPI spectra, the $\mathrm{A}^{2} \Sigma^{+}(v=0) \leftarrow \mathrm{X}^{2} \Pi_{\Omega}(v=2)$ and $\mathrm{A}^{2} \Sigma^{+}(v=0) \leftarrow$ $\mathrm{X}^{2} \Pi_{\Omega}(v=3)$ bands are well separated. The many lines in the spectra belong to the different rotational branches in these bands. Even a visual inspection of the spectra reveals strong effects of the incident orientation and translational energy. Firstly, the $\mathrm{A}^{2} \Sigma^{+}(v=0) \leftarrow \mathrm{X}^{2} \Pi_{\Omega}(v=2)$ band gains significantly in intensity compared to the $\mathrm{A}^{2} \Sigma^{+}(v=0) \leftarrow \mathrm{X}^{2} \Pi_{\Omega}(v=3)$ band when the translational energy is increased. This 


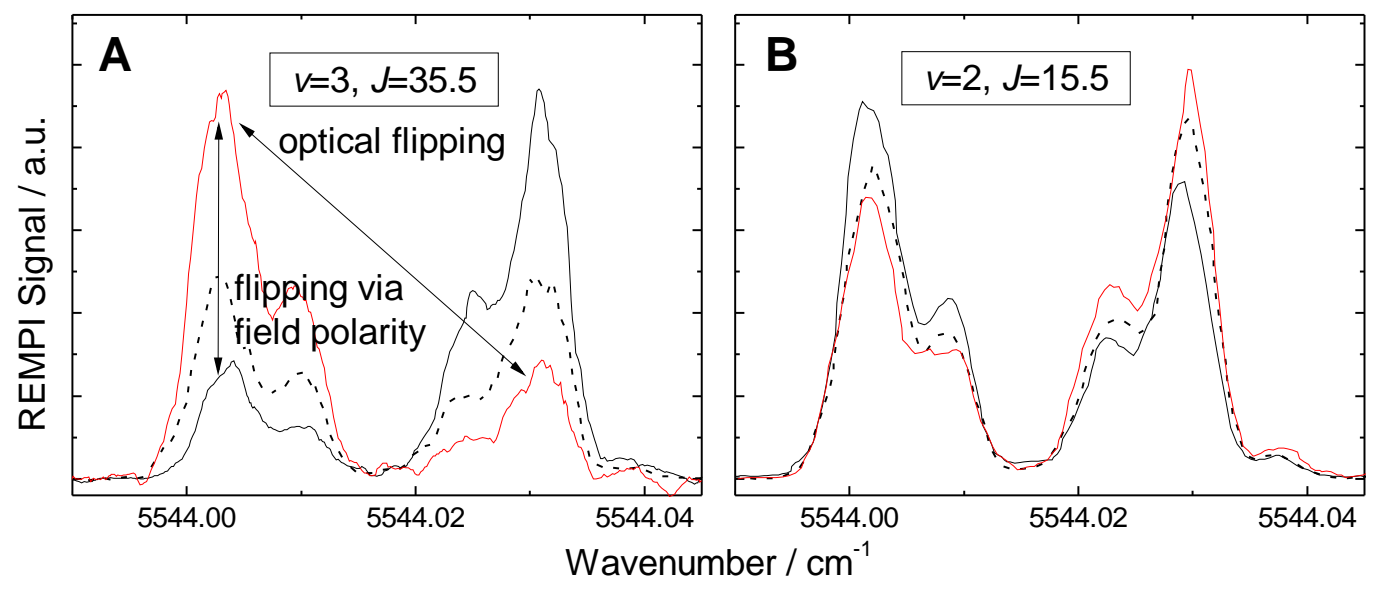

Figure 6.3: Orientation dependence of NO scattering from $\mathrm{Au}(111)$ probed for different ro-vibrational states. - Just as in Fig. 6.2 the population of specific rovibrational states of surface scattered molecules is probed by resonant ionization, while the IR laser is scanned over the (3-0) $\mathrm{Q}_{11}(0.5)$ transition. Measurements were performed with an orienting field of $+21.4 \mathrm{kV} / \mathrm{cm}$ (positive voltage on the electrode, red solid line), -21.4 $\mathrm{kV} / \mathrm{cm}$ (black solid line), and field free (black dashed line). In panel A, the REMPI laser probes the population in the $(v=3, J=35.5)$ level (which belongs to the vibrationally elastic $v=3 \rightarrow 3$ channel), and in panel B the $(v=2, J=15.5)$ level (vibrationally inelastic channel). The orientiation in the molecule can be changed by either tuning the IR laser to the other parity component of the $\Lambda$-doublet ("optical flipping") or by changing the field polarity ("flipping via field polarity"). Figure adapted from Ref. (4). 


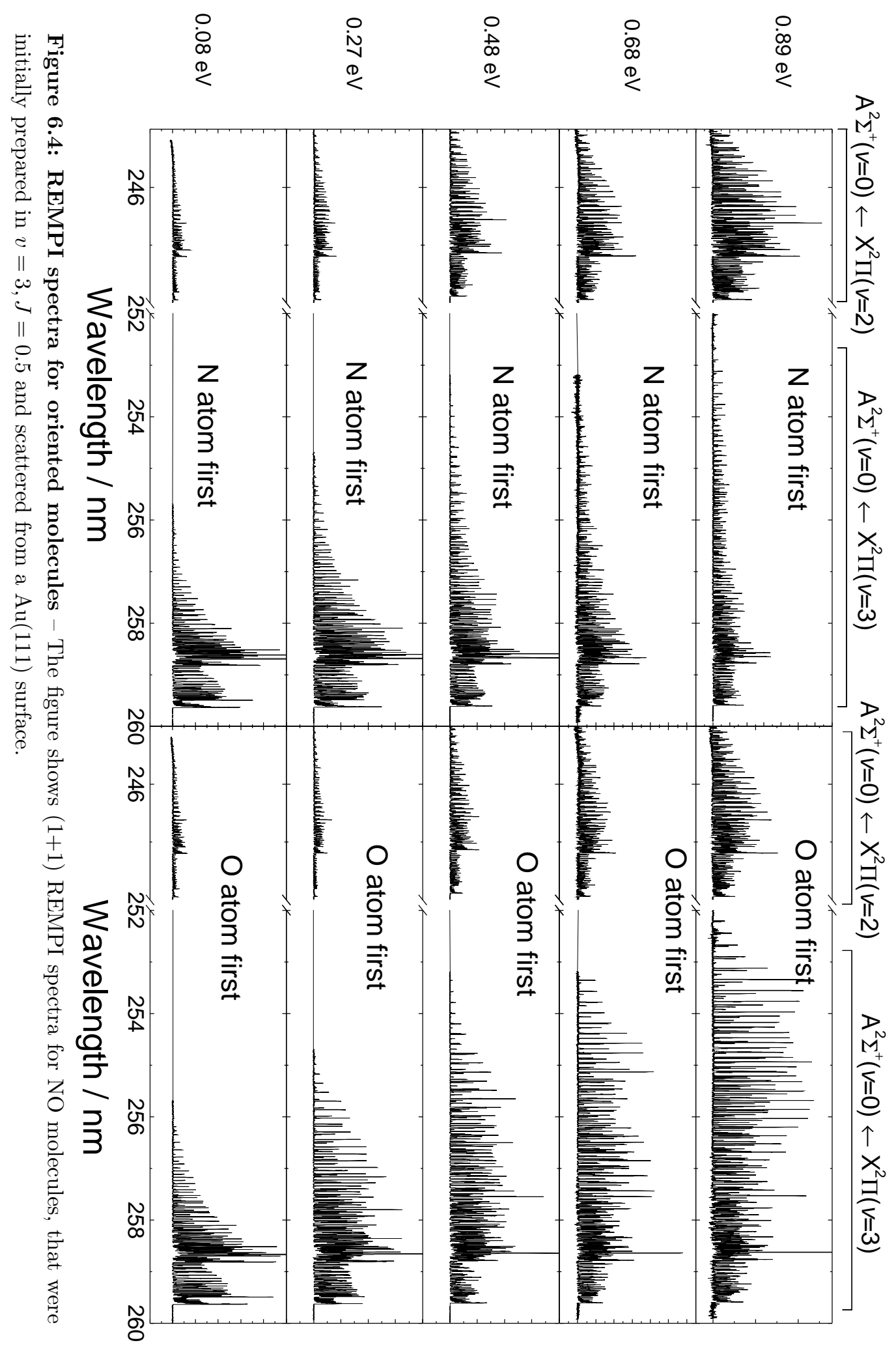


means, that the vibrational relaxation probability $\mathrm{NO}(v=3 \rightarrow 2)$ increases with incidence translation. This effect has been known previously from experiments on the "Beamer 1" setup (10, 56), see Fig. 2.9. However secondly, the intensity of the $\mathrm{A}^{2} \Sigma^{+}(v=0) \leftarrow \mathrm{X}^{2} \Pi_{\Omega}(v=2)$ band is also enhanced by $\mathrm{N}$-first orientation, meaning that the relaxation probability is higher for this orientation. Thirdly, the rotational structure of the $\mathrm{A}^{2} \Sigma^{+}(v=0) \leftarrow \mathrm{X}^{2} \Pi_{\Omega}(v=3)$ band strongly depends on the incidence translation and orientation. This effect is not observed for molecules that underwent vibrational relaxation to $v=2$ and are probed in the $\mathrm{A}^{2} \Sigma^{+}(v=0) \leftarrow \mathrm{X}^{2} \Pi_{\Omega}(v=2)$ band.

\subsubsection{Analysis of ro-vibrational state distributions}

Fig. 6.5 shows the rotational state distributions obtained by analyzing the REMPI spectra presented in Fig. 6.4. The analysis is performed by fitting individual nonoverlapping rotational lines. The intensities are corrected for the different rotational branches for Hönl-London factores, intermediate state alignment and partial saturation effects using the expression of Jacobs, Madix and Zare(145, 146) 11 Furthermore, I applied a density-to-flux correction. The density-to-flux correction accounts for that REMPI detection is sensitive to the density of the molecules, which has to be multiplied by the speed of the molecules to obtain a flux. Unfortunately, we cannot measure scattered speed distributions of the oriented scattered molecules. However, work from Golibrzuch et al. (10, 149) showed that the translational energy of molecules scattered from $\mathrm{Au}(111)$ with isotropic incidence orientation distributions is slightly enhanced by vibrational relaxation (translation-vibration coupling) and reduced upon rotational

\footnotetext{
${ }^{1}$ The exact treatment by Jacobs, Madix and Zare requires measuring REMPI spectra under isopower-conditions, meaning that several data pairs of laserpower and REMPI signal need to be recorded at each wavelength and the intensity is interpolated to a fixed laser power. In this experiment, the REMPI laser was instead continuously scanned and we correct for an experimentally derived linear power dependence at each data point. The correction was done assuming a photo-ionization cross section $\sigma$ of $7 \cdot 10^{-19} \mathrm{~cm}^{2}(147)$ with a fraction of parallel character $\Gamma=0.446$ (145), and an average laser power of $11 \mathrm{~mJ} \mathrm{~cm}{ }^{-2}$. The proportionality constant $C_{01}$ was determined to $1 \mathrm{~cm}^{2} / \mathrm{mJ}$ and the Hönl-London factors were calculated from Ref. (148). The correction was tested with REMPI spectroscopy on a thermal $\mathrm{NO}(v=0)$ rotational state distribution generated by leaking $\mathrm{NO}$ into the chamber. The correction leads to an overlap of corrected peak intensities belonging to the same rovibrational and spin-orbit state. This allows for a better averaging of the data from different rotational branches, but has small impact on the final rotational state distributions a depicted in Fig. 6.6
} 
excitation (translation-rotation coupling). The experimental data is over the range between reasonably well reproduced by the formula

$$
E_{\text {trans }}^{\mathrm{f}} / \mathrm{eV}=A \cdot E_{\text {trans }}^{\mathrm{i}} / \mathrm{eV}+m_{T V}\left[E_{\mathrm{vib}}^{\mathrm{i}}-E_{\mathrm{vib}}^{\mathrm{f}}\right] / \mathrm{eV}+m_{T R}\left[E_{\mathrm{rot}}^{\mathrm{f}}\right] / \mathrm{eV},
$$

where $E_{\mathrm{trans}}^{\mathrm{f}}$ and $E_{\mathrm{trans}}^{\mathrm{i}}$ are the final and incidence translational energies, $A=0.601$ is a constant close to the Baule limit, $E_{\text {rot }}^{\mathrm{f}}$ is the final rotational energy, and $E_{\mathrm{vib}}^{\mathrm{f}}$ and $E_{\mathrm{vib}}^{\mathrm{i}}$ are the final and incidence vibrational energies, respectively (energies in electron Volt, eV). The values for the translation-vibration coupling $m_{\mathrm{TR}}$ and the translationrotation coupling $m_{\mathrm{TV}}$ are approximately given by

$$
\begin{aligned}
& m_{\mathrm{TR}}=-0.575+0.631 \cdot \exp \left[-\frac{E_{\mathrm{trans}}^{\mathrm{i}}}{\mathrm{eV} \cdot 0.20834}\right] \\
& m_{\mathrm{TV}}=+0.01881+0.2091 \cdot E_{\mathrm{trans}}^{\mathrm{i}} / \mathrm{eV} .
\end{aligned}
$$

The density-to-flux conversion is performed by multiplying the measured peak intensities by the square-root of $E_{\text {trans }}^{\mathrm{f}}$ which is proportional to the speed of the scattered molecules. The density-to-flux conversion has almost negligible effect on the final vibrational state distributions. In the rotational state distributions, the population of high rotational states is slightly shifted to lower values compared to the population of low rotational states.

There are several important observations to note in the rotational state distributions from Fig. 6.5. Firstly, the rotational state distributions in the vibrationally elastic channel (panels (a) to (e)) show very pronounced rotational rainbow structure (intensity peak at high $J$ ). These rotational rainbows are observed in both spin-orbit states (population in $\Omega=1 / 2$ and $\Omega=3 / 2$ are given with solid and open symbols, respectively), and are most heavily pronounced for $\mathrm{O}$-atom first collisions (red symbols) and weak for $\mathrm{N}$-atom first collisions (blue symbols). Furthermore, the maximum of the O-first rainbow shifts from approximately $J=40.5$ at an incidence translational energy of $0.89 \mathrm{eV}$ to lower $J$-states with decreasing incidence translation. At the lowest measured incidence translational energy $(0.08 \mathrm{eV})$ no roational rainbow is observed. In contrast, rotational state distributions of molecules scattered in the vibrationally inelastic channel ( $v=3 \rightarrow 2$, panels (f) to (j)) have no rotational rainbow structure for any incidence orientation or translational energy. The relative population of the 


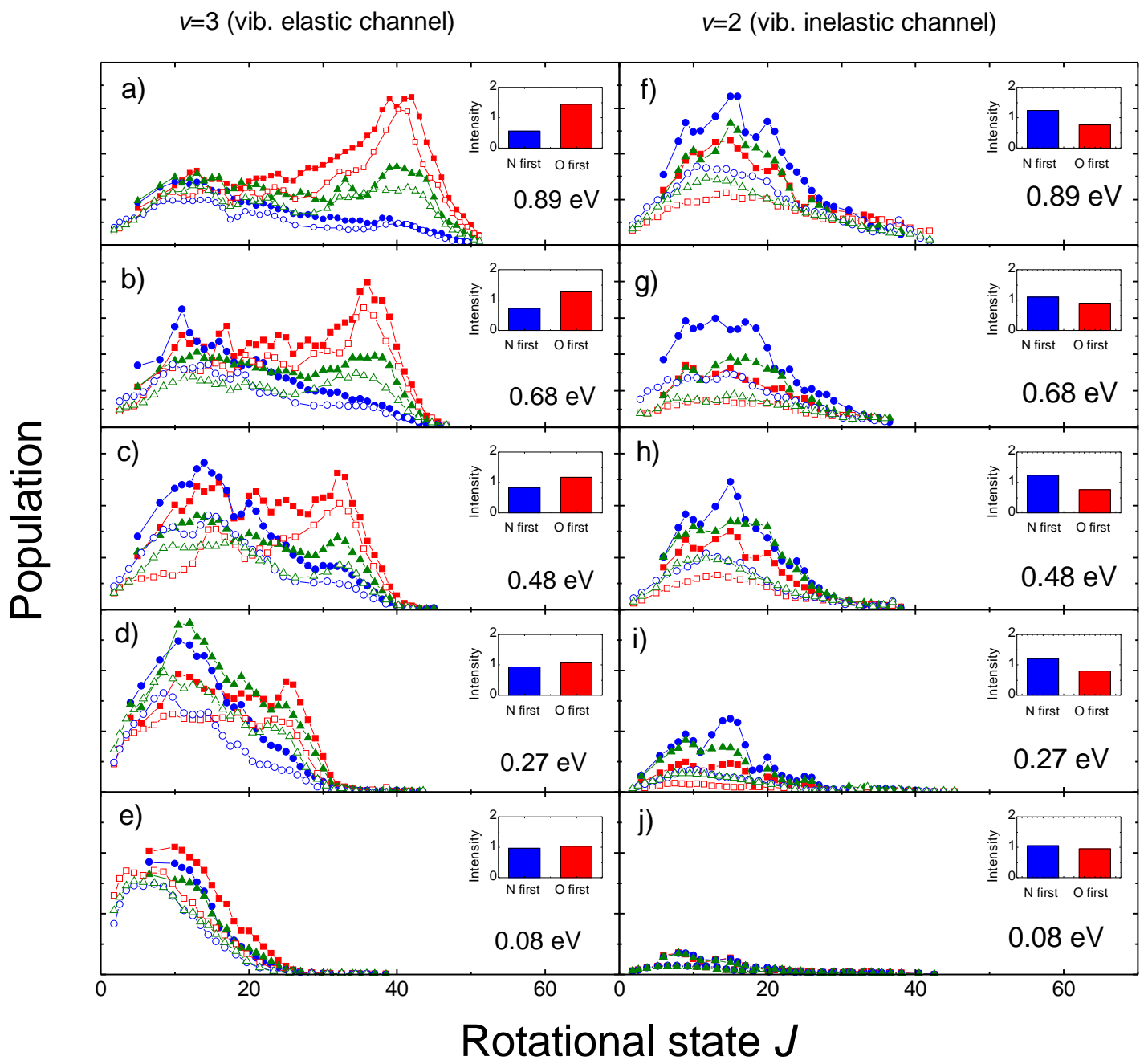

Figure 6.5: Experimentally derived rotational state distributions for NO $\mathrm{X}^{2} \Pi_{1 / 2}(v=3, J=0.5)$ scattered from $\mathrm{Au}(\mathbf{1 1 1})$ - Measurements were performed at five different incidence translational energies given as labels. The colors denote $\mathrm{N}$-first (blue), isotropic (green), and O-first (red) orientation of incident molecules. The filled symbols represent population in the $\Omega=1 / 2$ and open symbols in the $\Omega=3 / 2$ spin-orbit state. Whereas molecules scattered in the vibrationally elastic channel $v=3 \rightarrow 3$ show pronounced rotational rainbows, these rainbows do not appear in the $v=3 \rightarrow 2$ relaxation channel. The insets show the integrated band intensities (relative to the band intensities for isotropic scattering) for both orientations and thus reflect the relative survival and relaxation probabilities. A simplified figure only showing data on the $\Omega=1 / 2$ state without a density-to-flux conversion has been published as Fig. 5 in Ref. (5). Unfortunately, the original figure was reported with an error, as the population distributions were converted from the representation as function of $J$ to a representation as function of the rotational energy without accounting for the appropriate Jacobian. 
vibrational states $v=3$ and $v=2$ for different orientations can be derived by integrating over the entire band intensities of the $\mathrm{A}^{2} \Sigma^{+}(v=0) \leftarrow \mathrm{X}^{2} \Pi_{\Omega}(v=3)$ and $\mathrm{A}^{2} \Sigma^{+}(v=0) \leftarrow \mathrm{X}^{2} \Pi_{\Omega}(v=2)$ bands, respectively. These band intensities were normalized to 1 for the molecules scattered with an isotropic incidence orientation distribution and are given as insets in Fig. 6.5. It is found, that vibrational relaxation is clearly enhanced by an NO orientation in which the $\mathrm{N}$-atom is pointing towards the surface.

\subsubsection{Rotational cooling upon vibrational relaxation}

Fig. 6.6 shows the mean rotational energy $E_{\text {rot }}^{\mathrm{f}}$ of the initially non-rotating surface scattered molecules as function of the incidence translational energy.

The rotational energy in the vibrationally elastic channel $(v=3 \rightarrow 3)$ is strongly dependent on the incidence translation and is higher for $\mathrm{O}$-first collisions, than for $\mathrm{N}$-first collisions. As opposed to this, molecules in the vibrationally inelastic channel $(v=3 \rightarrow 2)$ are rotationally much colder, except at the lowest incidence energy of translation. It should be pointed out that (a least above the lowest measured translational energy) we can exclude that this is the result of a trapping-desorption mechanism, as scattered molecules were found to 1) have narrow angular distributions 2) scatter from the surface in accordance with specular scattering, and 3) generally have higher translational and rotational energy than expected for a thermalization with the surface $\left(k_{\mathrm{B}} T=0.026 \mathrm{eV}\right.$ at room temperature). The effect that molecules that underwent vibrational relaxation are rotationally colder compared to vibrational elastically scattered molecules is known as rotational cooling upon vibrational relaxation and will be discussed together with the rotational rainbow structure in more detail in section 6.3.2.

\subsubsection{Incidence energy of translation dependence of the steric effect}

Following previous work, it is also possible to describe the steric effect in terms of an asymmetry parameter $A$ defined as

$$
A=\frac{I_{\mathrm{O}}-I_{\mathrm{N}}}{I_{\mathrm{O}}+I_{\mathrm{N}}},
$$

where $I_{\mathrm{O}}$ and $I_{\mathrm{N}}$ are the populations produced by $\mathrm{O}$-atom first and $\mathrm{N}$-atom first collisions, respectively. Positive values of the asymmetry parameter (the maximum value of $A$ is +1 ) indicate that population in the probed state is enhanced for $\mathrm{O}$-first 


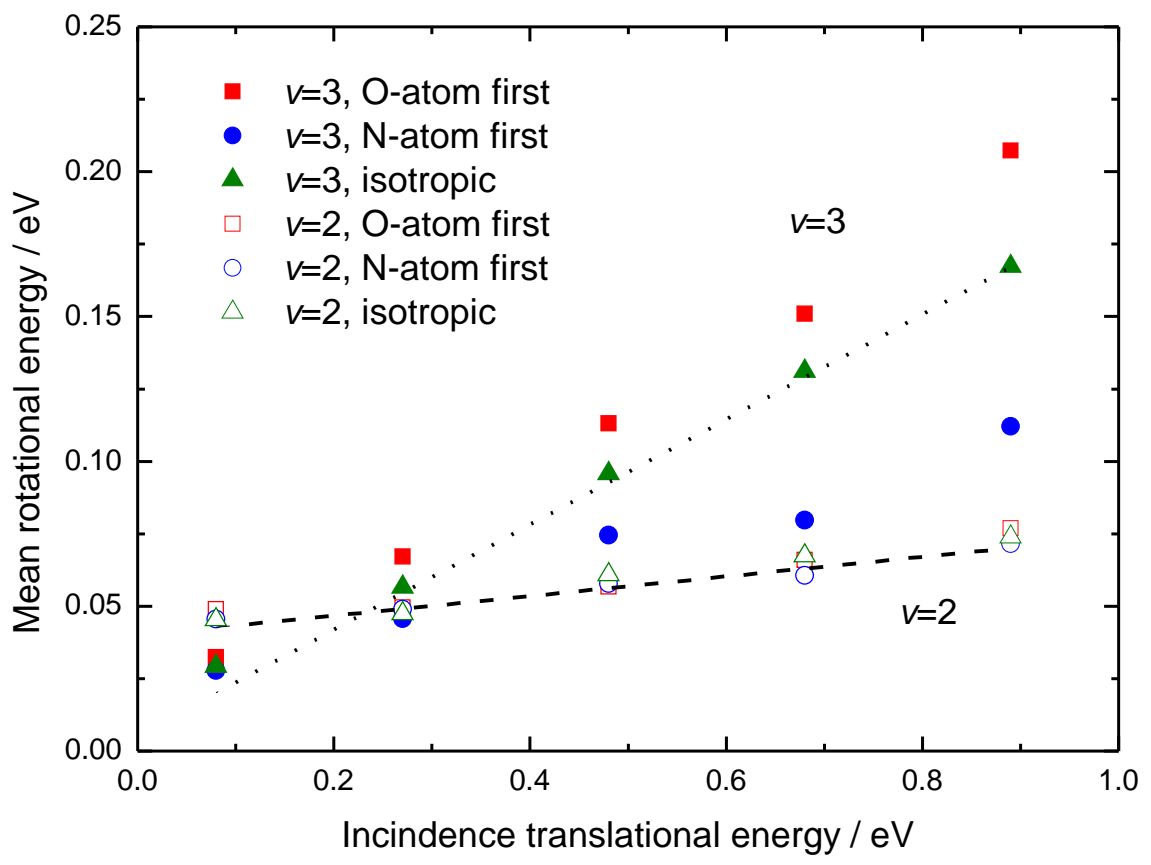

Figure 6.6: Mean rotational energies - Average rotational energies of initially nonrotating NO in vibrationally elastic $(v=3 \rightarrow 3)$ and vibrationally inelastic $(v=3 \rightarrow 2)$ collisions with $\mathrm{Au}(111)$ as function of the incidence translational energy and incidence orientation. The colors denote scattering with $\mathrm{N}$-first (blue), isotropic (green) and $\mathrm{O}-$ first (red) orientation. Lines are drawn to guide the eye. The rotational energy in the vibrationally elastic channel $(v=3 \rightarrow 3)$ strongly increases with the incidence translation, whereas the rotational energy of inelastically scattered molecules $(v=3 \rightarrow 2)$ increases only little with the incidence translation. The figure is adapted from Fig. 7 of Ref. (5), but now includes the density-to-flux correction. Unfortunately, the original figure was reported with an error, as the mean rotational energies were calculated from rotational state distributions generated without using the appropriate Jacobian. 
collisions, while negative values (the minimum value of $A$ is -1 ) represent an enhanced population by $\mathrm{N}$-first collsions.

Fig. 6.7 shows asymmetry parameters for the vibrational states $v=3,2$ and 1 as

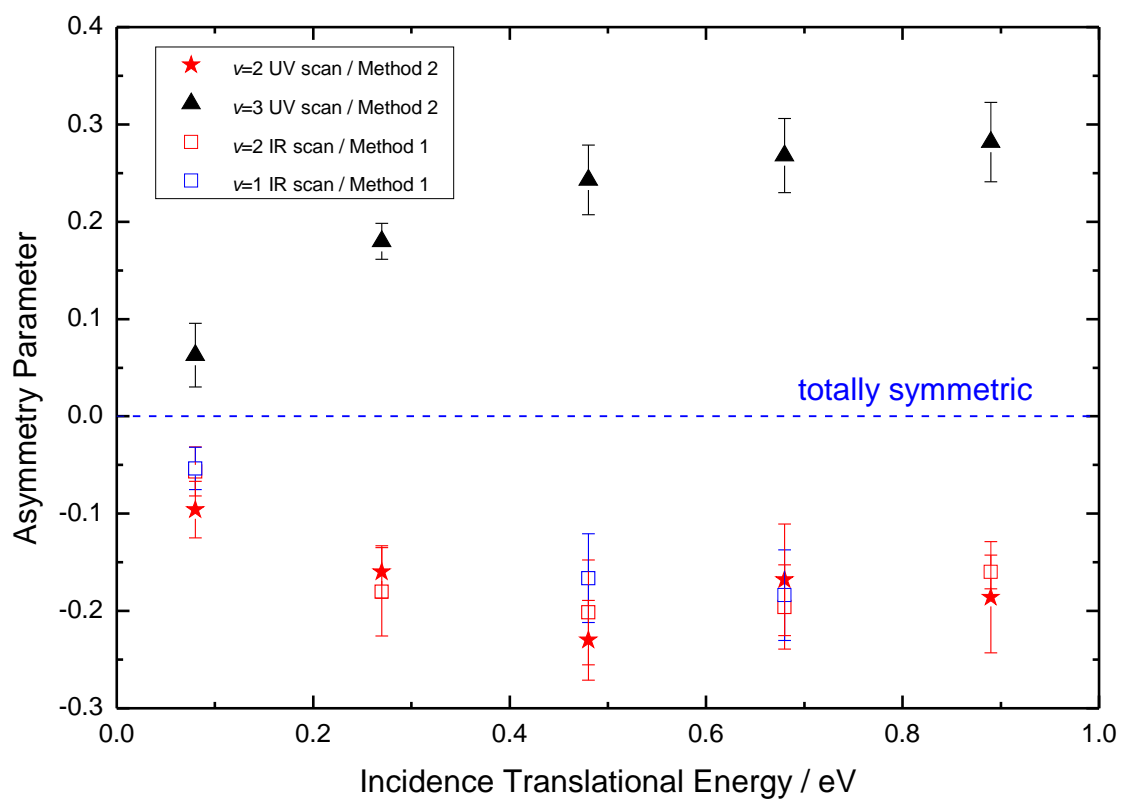

Figure 6.7: Experimentally derived asymmetry parameters - The asymmetry parameters $A=\left(I_{\mathrm{O}}-I_{\mathrm{N}}\right) /\left(I_{\mathrm{O}}+I_{\mathrm{N}}\right)$ are given as function of the incidence translational energy. Molecules were initially prepared in $\mathrm{NO} \mathrm{X}{ }^{2} \Pi_{1 / 2}(v=3, J=0.5)$ and scattered from a clean $\mathrm{Au}(111)$ surface at normal incidence. Reproduced with permission from (5). Copyright 2014 American Chemical Society.

function of the incidence translational energy. The asymmetry parameters given as black triangles (for $v=3$ ) and red stars (for $v=2$ ) are calculated from the integrated band intensities for the $\mathrm{A}^{2} \Sigma^{+}(v=0) \leftarrow \mathrm{X}^{2} \Pi_{\Omega}(v=3)$ and $\mathrm{A}^{2} \Sigma^{+}(v=0) \leftarrow \mathrm{X}^{2} \Pi_{\Omega}(v=$ 2) bands, which have already been given as insets in Fig. 6.5. Unfortunately, it is difficult to evaluate the asymmetry parameter in $v=1$ for this method, because REMPI spectra have a large background from thermally populated $v=1$ that is scattered in the $v=1 \rightarrow 1$ channel. It is however possible to determine asymmetry parameters for single rotational states with IR scans in method 1 as shown in Fig. 6.3. This has the advantage, that scanning the IR laser back and forth over the $\Lambda$-doublet allows for a repeated flipping of the orientation and good averaging of the small signal on top of the large background. By measuring the asymmetry parameters on several rotational lines 
and weighing the results assuming a similar rotational state distributions for $v=2$ and $v=1$ it is possible to derive asymmetry parameters for the vibrational state. These are given as red and blue open squares for $v=2$ and 1 , respectively.

It is found that the asymmetry parameters for the $v=3 \rightarrow 3$ channel are positive and the asymmetry parameters for the vibrationallly inelastic $v=3 \rightarrow 2$ and $v=3 \rightarrow 1$ channels are negative for all incidence translational energies. This reflects the higher $v=3$ survival probability for $\mathrm{O}-$ first collisions and the corresponding higher relaxation probability for $\mathrm{N}$-first collisions. Interestingly, the asymmetry parameters in the $v=$ $3 \rightarrow 2$ and $v=3 \rightarrow 1$ are constant over the range of $0.27 \mathrm{eV}$ and $0.89 \mathrm{eV}$, but are much weaker for the lowest incidence translational energy of $0.08 \mathrm{eV}$. In section 6.3.3 I will argue, that this effect might be the result of a dynamical steering process in which forces between the molecule and the surface reorient the molecule prior to the collision.

\subsubsection{Incomplete equilibration of spin-orbit states}

Fig. 6.8 shows the ratio of the population in the $\Omega=1 / 2$ spin-orbit state $\mathrm{X}^{2} \Pi_{1 / 2}(v)$ to the population in the $\Omega=3 / 2$ spin-orbit state $\mathrm{X}^{2} \Pi_{3 / 2}(v)$ for different orientations and vibrational states as function of the incidence translational energy. It is found, that the population in the $\Omega=1 / 2$ state is under all conditions (orientation, vibrational state) higher than in the $\Omega=3 / 2$ state, which is approximately $120 \mathrm{~cm}^{-1}(0.0149 \mathrm{eV})$ lower in energy.

In the $v=3 \rightarrow 3$ channel, the spin-orbit population ratio is found to be rather independent of the incidence orientation and translational energy and has a value of $\approx 1.5$. The spin-orbit population ratio in the $v=3 \rightarrow 2$ channel is even higher and decreases with incidence translational energy in the range of $0.27 \mathrm{eV}$ to $0.89 \mathrm{eV}$. 


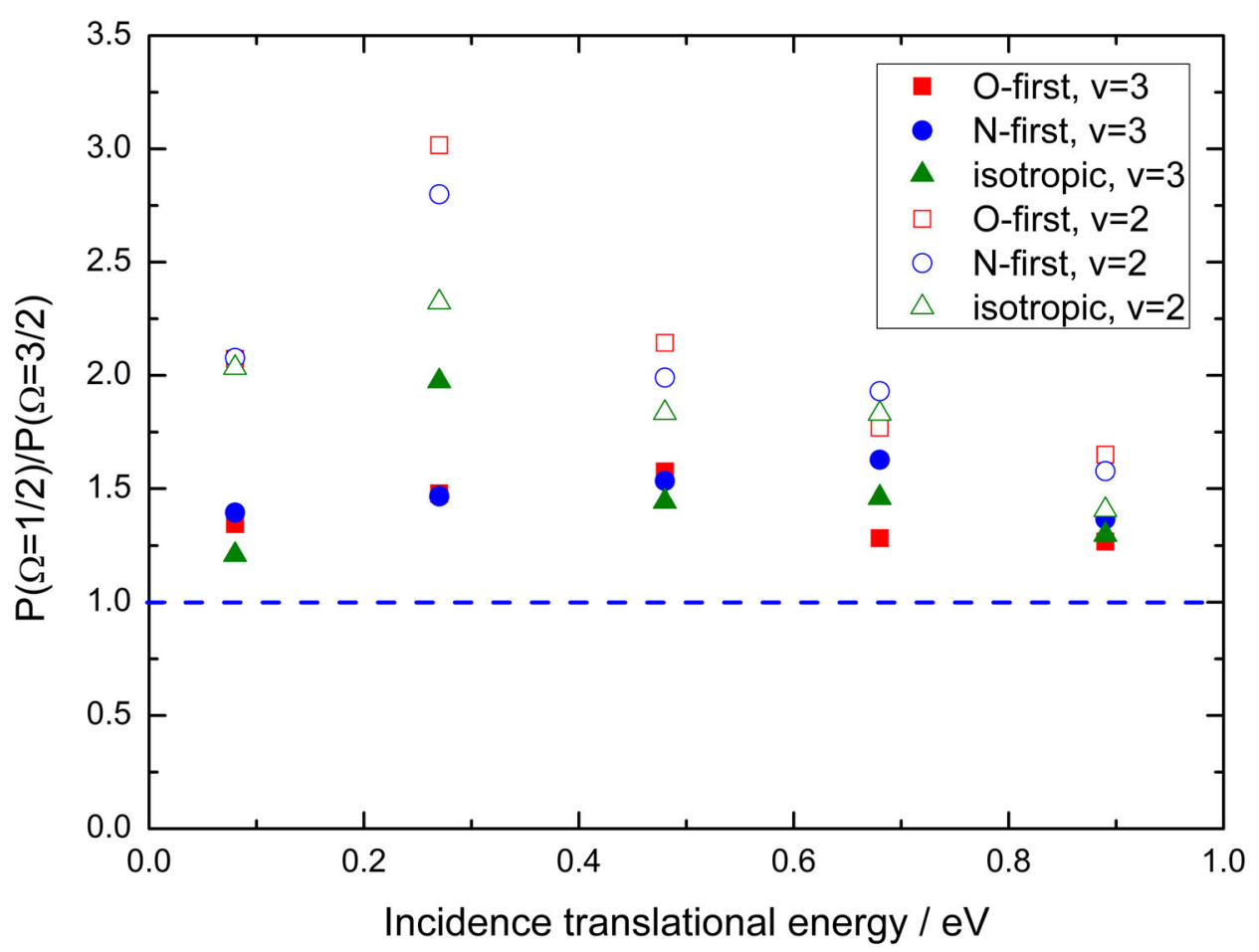

Figure 6.8: Population ratio in the spin-orbit states - Population ratio of the $\Omega=1 / 2$ and $\Omega=3 / 2$ spin-orbit from an interpolation of experimental data from Fig. 6.5 . Molecules were initially prepared in $\mathrm{NO} \mathrm{X}^{2} \Pi_{1 / 2}(v=3, J=0.5)$ and scattered from a clean $\mathrm{Au}(111)$ surface at normal incidence. The blue dashed line indicates equal population in both states. 


\subsection{Vibrationally inelastic scattering of NO prepared in high vibrational states from $\mathrm{Au}(111)$}

\subsubsection{Experimental overview}

Fig. 6.9 shows a schematic of the experiment for the vibrationally inelastic scattering of highly vibrationally excited NO from $\mathrm{Au}(111)$.

Mixtures of NO seeded in a carrier gas (see table 6.1) were expanded into vacuum in a

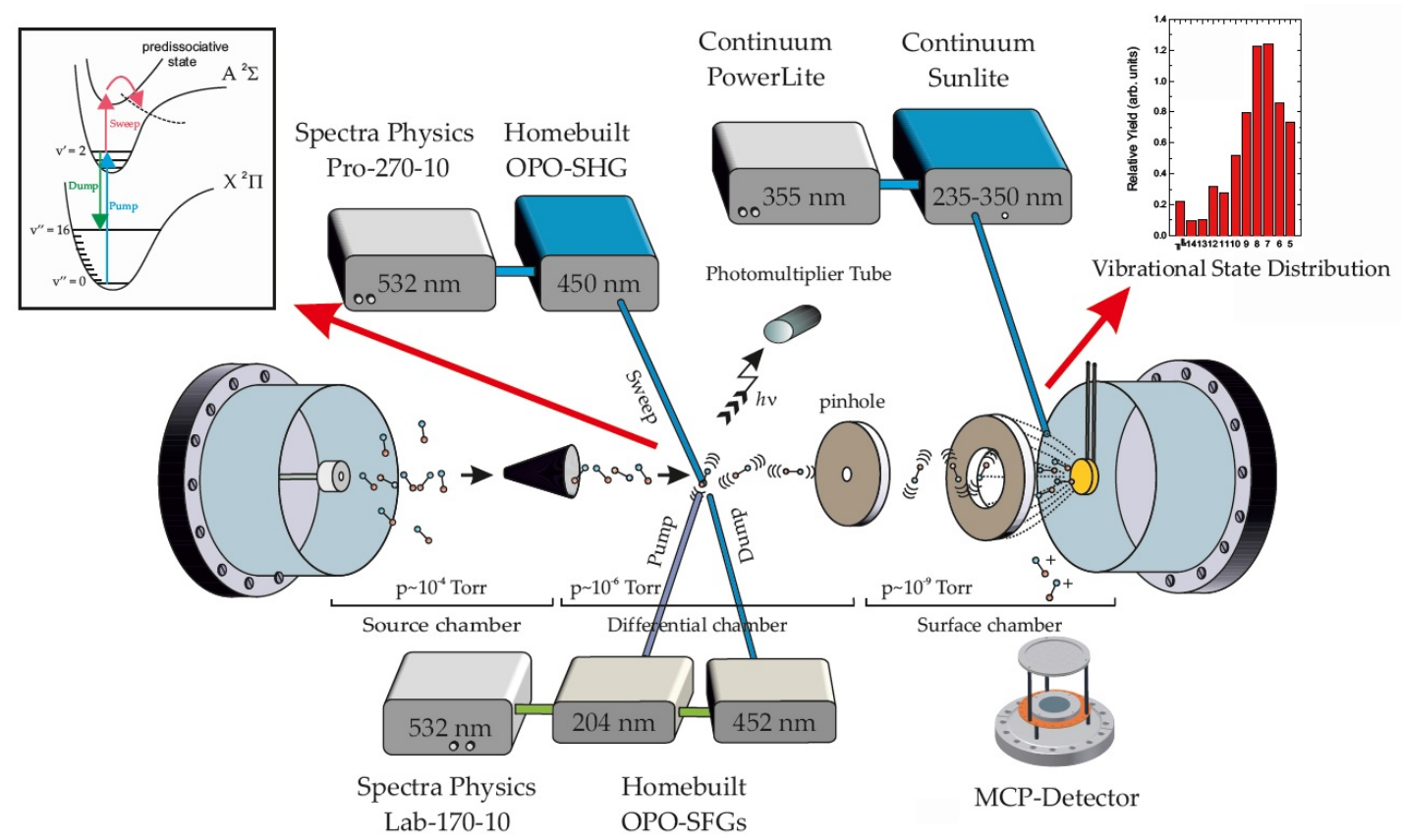

Figure 6.9: Schematic of the vibrational relaxation scattering experiment for NO prepared in high vibrational states - NO molecules are prepared in a high vibrational state using three laser pulses in the pump-dump-sweep technique. Then, molecules scatter from the clean $\mathrm{Au}(111)$ surface at normal incidence. After scattering, vibrational state distributions are probed with REMPI spectroscopy. It is also possible to orient NO molecules prior to the collision by applying a high voltage to the orientation electrode (shown as a ring electrode, a better drawing is given in Fig. 3.1.). This electric field is pulsed to ground after scattering, but prior to REMPI detection.

supersonic jet expansion. Molecules were prepared in a highly excited vibrational state $\left(v_{\mathrm{i}}=11\right.$ or 16$)$ of $\mathrm{NO} \mathrm{X}^{2} \Pi_{1 / 2}\left(v_{\mathrm{i}}, J=0.5, f\right)$ in the differential chamber with the pumpdump-sweep technique. Pump-dump-sweep was performed using three laser pulses. First, electronic excitation (pump step) was performed with the $Q_{11}(0.5)$ transition of 
the $\mathrm{A}^{2} \Sigma^{+}\left(v^{\prime}=2\right) \leftarrow \mathrm{X}^{2} \Pi_{1 / 2}\left(v^{\prime \prime}=0\right)$ band at $204.708 \mathrm{~nm}\left(\lambda_{1}\right)$ using one of the homebuilt OPO laser systems $(0.5 \mathrm{~mJ} /$ pulse, $3 \mathrm{~mm}$ beam diameter $)$. Second, molecules were de-excited (dump step) with the second home-built OPO laser system $(1 \mathrm{~mJ} / \mathrm{pulse}$, $4 \mathrm{~mm}$ beam diameter $)$ via the $Q_{11}(0.5)$ transition of $\mathrm{A}^{2} \Sigma^{+}\left(v^{\prime}=2\right) \rightarrow \mathrm{X}^{2} \Pi_{1 / 2}\left(v^{\prime \prime}\right)$ at $\lambda_{2}=450.503 \mathrm{~nm}$ for $v^{\prime \prime}=16$ or at $\lambda_{2}=336.101 \mathrm{~nm}$ for $v^{\prime \prime}=11$, respectively. Subsequently, we used the same sweep transition $\left(\lambda_{3}=450.87 \mathrm{~nm}\right)$ as described in chapter 5 to suppress fluorescence from the $\mathrm{A}^{2} \Sigma^{+}\left(v^{\prime}=2\right)$ state. The sweep laser pulse $(\approx 8$ $\mathrm{mJ} /$ pulse, $5 \mathrm{~mm}$ diamter) was fired $13 \mathrm{~ns}$ after the pump and dump pulses. The pump and dump pulses were overlapped in time.

In order to obtain information about all possible $v \rightarrow v$ scattering channels, we measured REMPI spectra of surface scattered molecules over wide ranges of wavelengths (between $235 \mathrm{~nm}$ and $315 \mathrm{~nm}$ for $v_{\mathrm{i}}=11$ and between $245 \mathrm{~nm}$ and $350 \mathrm{~nm}$ for $v_{\mathrm{i}}=16$ ) using the second harmonic of the Sunlite OPO (Continuum, $\approx 1 \mathrm{~mJ} /$ pulse, $2 \mathrm{~mm}$ beam diameter). REMPI spectra were measured in wavelength ranges of $5 \mathrm{~nm}$ per scan at a scanning speed of $0.01 \mathrm{~nm} / \mathrm{s}$. We also recorded background spectra (with blocked dump pulse).

In practice, measuring an entire $245 \mathrm{~nm}$ to $350 \mathrm{~nm}$ spectrum with the corresponding background required approximately $2-3$ subsequent days. We thus took measures to account for long-term drifts in the signal intensity of the four laser experiment. This was achieved by regularly measuring $1 \mathrm{~nm}$ reference REMPI scans probing always the same wavelength range. Then the signal intensities of the measured $5 \mathrm{~nm}$ scans were corrected for the average intensity of the corresponding reference scan.

We also performed scattering experiments with oriented molecules. Because of the small dipole moment of NO in $v=11$ (compare Fig. 4.7), we used a higher electric orientation field strength of $\pm 33 \mathrm{kV} / \mathrm{cm}(15 \mathrm{kV}$ at $4.5 \mathrm{~mm}$ distance between the electrode and the surface) compared to the $v=3$ scattering experiments. The molecules orientation was flipped by switching the polarity of the electric field. We always measured REMPI spectra for both orientations as well as signal and background spectra in each $5 \mathrm{~nm}$ wavelength interval before moving to the next wavelength interval (again to minimize errors from long-term drifts). 


\subsubsection{Example of a REMPI spectrum}

During the collision of highly vibrationally excited and initially non-rotating NO molecules with the $\mathrm{Au}(111)$ surface, the incident population is transferred to many different ro-vibrational states. This leads to very congested REMPI spectra when probing surface scattered molecules. Fig. 6.10 shows the REMPI spectrum detecting scattered $\mathrm{NO}$ molecules after the collision of $\mathrm{NO} \mathrm{X}^{2} \Pi_{1 / 2}\left(v^{\prime \prime}=11, J=0.5\right)$ (isotropic incidence angular distribution, incidence translational energy of $0.51 \mathrm{eV}$ ) with the $\mathrm{Au}(111)$ surface. The top panel shows the four laser signal from the pump-dump-sweep and probe experiment. Almost the entire intensity in the REMPI spectrum results from $(1+1)$ ionization of different ro-vibrational states in $\mathrm{NO} \mathrm{X}^{2} \Pi$ via the $\mathrm{A}^{2} \Sigma^{+}$state (ionization via the $\gamma$-bands). We could not find any evidence for contributions from ionization via the $\mathrm{B}^{2} \Pi$ state (ionization via the $\beta$-bands). This was expected, since the $\mathrm{A}^{2} \Sigma^{+}$ state has a 40 times higher ionization cross section compared to the $\mathrm{B}^{2} \Pi$ state in the probed wavelength range 1 The middle panel shows the REMPI spectrum when the dump laser is blocked. This background spectrum has contributions due fluorescence from the $\mathrm{A}^{2} \Sigma^{+}\left(v^{\prime}=2\right)$ not suppressed by the sweep pulse. The distinct lines with high intensity in the background spectrum belong to the incident molecular beam, which is not completely separated from the scattered signal in time or space, because the population is probed very close to the surface. The $\gamma$-bands (1st step of the REMPI scheme) are often labelled according to the convention that $\gamma\left(v^{\prime}, v^{\prime \prime}\right)$ means that molecules in $\mathrm{X}^{2} \Pi\left(v^{\prime \prime}\right)$ are excited via $\mathrm{A}^{2} \Sigma^{+}\left(v^{\prime}\right) \leftarrow \mathrm{X}^{2} \Pi\left(v^{\prime \prime}\right)$. I marked line positions of the $\mathrm{Q}_{11}(0.5)$ and $\mathrm{Q}_{22}(1.5)$ lines of relevant $\gamma\left(v^{\prime}, v^{\prime \prime}\right)$-bands in the figure and labelled them according to this convention. The bottom panel shows an enlargement of a wavelength

\footnotetext{
${ }^{1}$ The difference in the ionization cross sections for the $\mathrm{A}^{2} \Sigma^{+}$and $\mathrm{B}^{2} \Pi$ states can be explained in terms of their dominant electronic configurations. The $\mathrm{A}^{2} \Sigma^{+}$state has the electronic configuration ... $(\sigma 2 p)^{2}(\pi 2 p)^{4}\left(\pi^{*} 2 p\right)^{0}\left(\sigma^{*} 2 p\right)^{1}$ and ionization simply removes the Rydberg electron $\ldots(\sigma 2 p)^{2}(\pi 2 p)^{4}\left(\pi^{*} 2 p\right)^{0}\left(\sigma^{*} 2 p\right)^{1} \rightarrow \ldots(\sigma 2 p)^{2}(\pi 2 p)^{4}+\mathrm{e}^{-}$. As opposed to this, ionization via the $\mathrm{B}^{2} \Pi$ state involves a valence-Rydberg excitation with a change of two electron orbitals: ... $(\sigma 2 p)^{2}(\pi 2 p)^{3}\left(\pi^{*} 2 p\right)^{2} \rightarrow$ $\ldots(\sigma 2 p)^{2}(\pi 2 p)^{4}+\mathrm{e}^{-} .(150)$ The ionization cross section of the $\mathrm{A}^{2} \Sigma^{+}$state varies relatively weakly with the intermediate vibrational state, e.g. $(7.0 \pm 0.9) 10^{-19} \mathrm{~cm}^{-1}$ for $\mathrm{A}^{2} \Sigma^{+}\left(v^{\prime}=0\right),(8.5 \pm 0.8) 10^{-19} \mathrm{~cm}^{-1}$ for $\mathrm{A}^{2} \Sigma^{+}\left(v^{\prime}=1\right)$ and $(6.0 \pm 1.0) 10^{-19} \mathrm{~cm}^{-1}$ for $\mathrm{A}^{2} \Sigma^{+}\left(v^{\prime}=2\right)$.(150) In addition, it has been reported to change only weakly with the ionization wavelength (for $\mathrm{A}^{2} \Sigma^{+}\left(v^{\prime}=0\right)$ the ionization cross section goes from $1.158 \cdot 10^{-18} \mathrm{~cm}^{-1}$ at $225 \mathrm{~nm}$ to $1.135 \cdot 10^{-19} \mathrm{~cm}^{-1}$ at $324 \mathrm{~nm}$ according to another reference (151) ). This insensitivity towards the intermediate vibrational state and the ionization wavelength makes REMPI spectroscopy via the $\mathrm{A}^{2} \Sigma^{+}$state of $\mathrm{NO}$ very suitable to probe vibrational state distributions.
} 


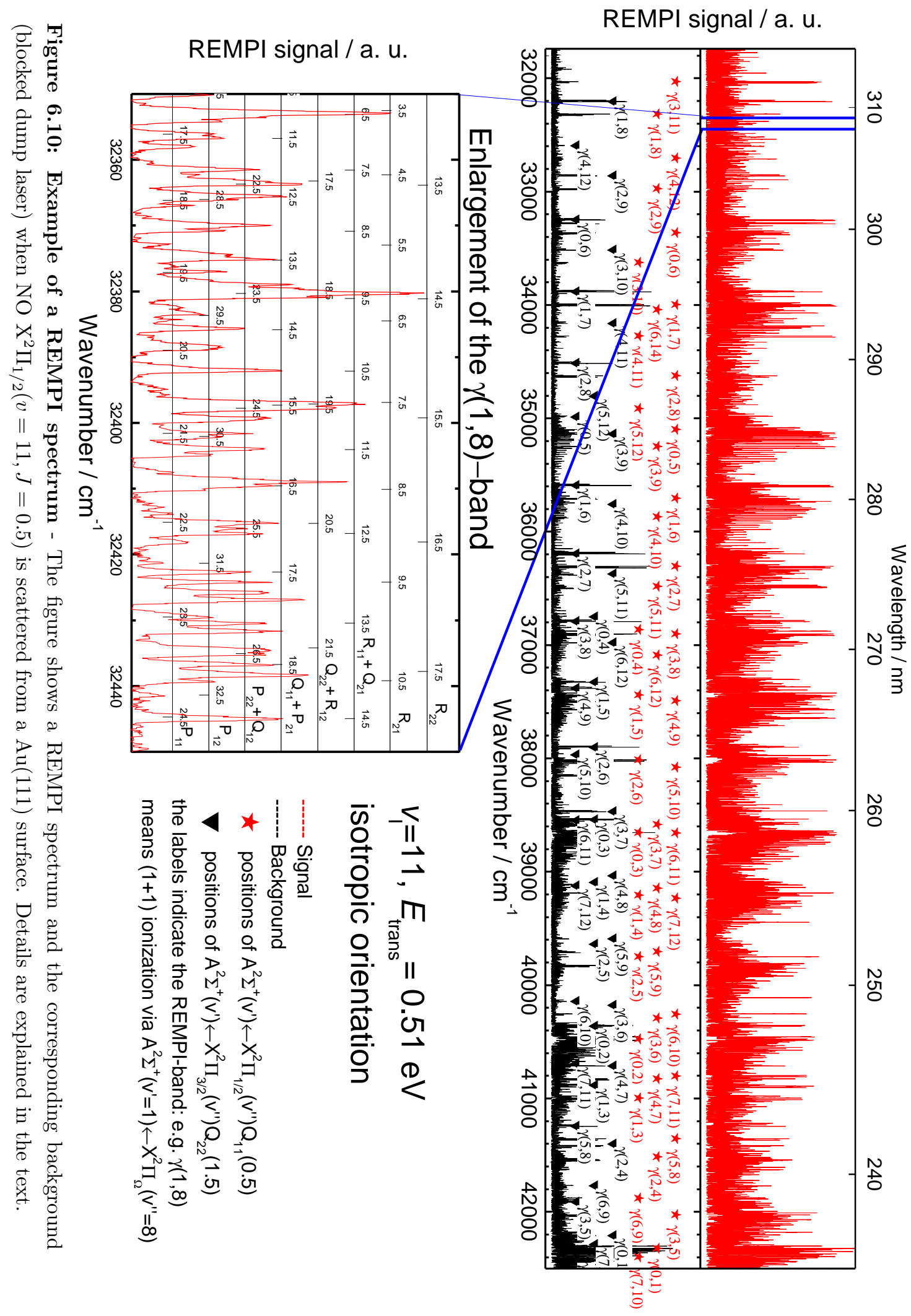


range in the signal spectrum mostly containing contributions from the $\gamma(1,8)$ band. The resolution is high enough to assign the rotational lines in the different rotational branches.

\subsubsection{Concept for the analysis of ro-vibrational state distributions}

The evaluation of rotational and vibrational state distributions from REMPI spectra as shown in Fig. 6.10 is a major task for computer assisted data analysis. The concept of this data analysis is schematically presented in Fig. 6.11. The entire data analysis is performed with Wolfram Mathematica. Further details on the data analysis and tables with vibrational state distributions can be found in the appendix.

In a first step (panel A), REMPI spectra are corrected for the photon density, which
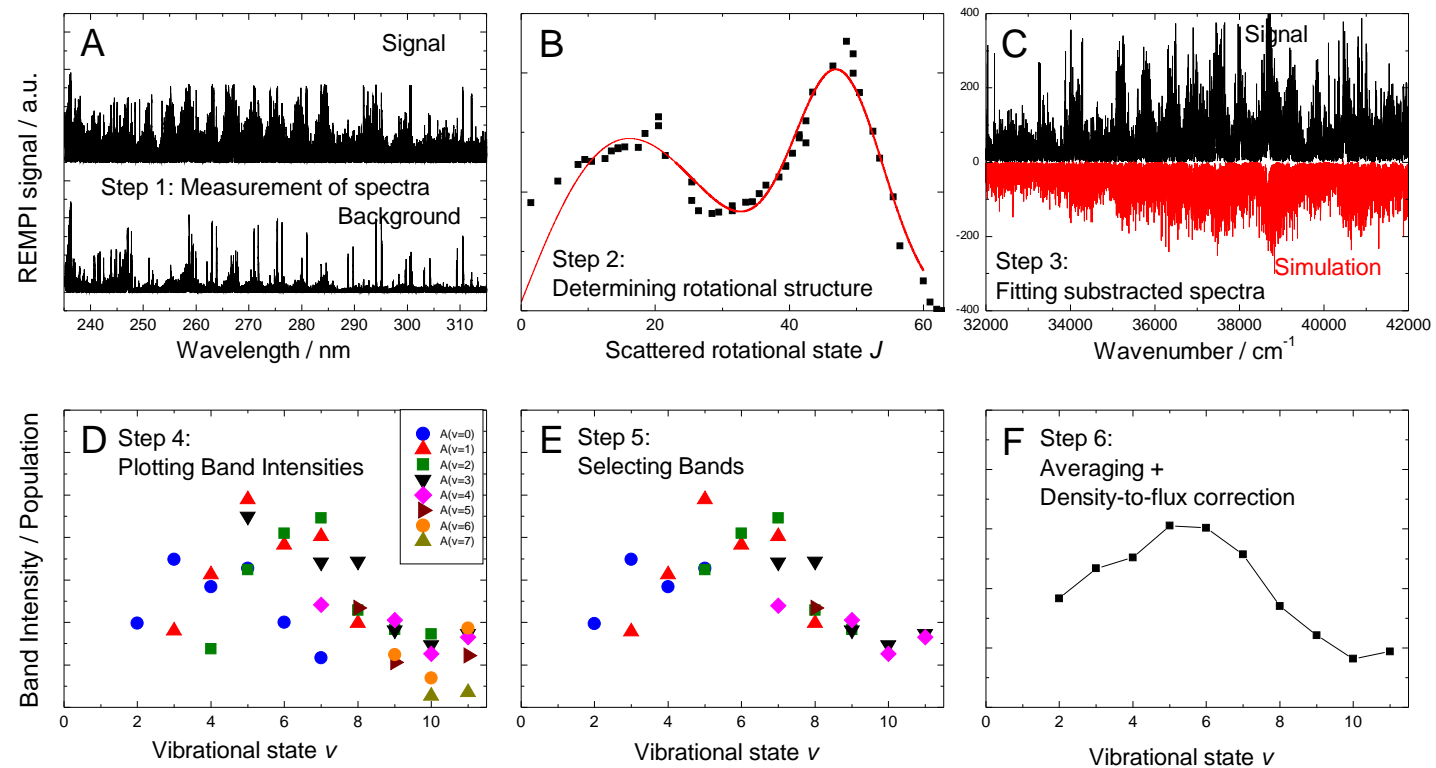

Figure 6.11: Steps in the data analysis for determining vibrational state distributions - The panels are explained in the text. The data shown belongs to a measurement for $v=11$ at an incidence translational energy of $0.51 \mathrm{eV}$ with an isotropic incidence angular distribution. Figure adapted from the appendix of Ref. (6).

means they are divided by the laser pulse energy and the wavelength.

In a second step (panel B), we determine rotational state distributions. This is done by fitting individual non-overlapping ro-vibrational transitions in the REMPI spectra. These transitions are found by calculating line positions of all transitions in the $\gamma-$ 
bands which can contribute to the REMPI spectra. Then a simple algorithm calculates which lines are not overlapped by other transitions within a defined critical minimum wavenumber range of $\pm 0.7 \mathrm{~cm}^{-1}$ around the line center. Thereby, the algorithm assumes no population to be present in higher vibrational states than $v=12$ for $v_{\mathrm{i}}=11$ and $v=16$ for $v_{\mathrm{i}}=16$ and above a maximum rotational state $J_{\max }$ that depends on the incidence translation energy $]^{1}$ The number of non-overlapping lines decreases for higher incidence translational energies (because rotational distributions become hotter), lower vibrational states (these are in general at lower wavelength, where more REMPI bands overlap, see Fig. 6.10) and for higher incidence vibrational states (because more vibrational states are populated). Nevertheless, by averaging data from different rotational branches similar to the analysis of the experiments for NO $(v=3)$ scattering, reasonable rotational state distributions can be obtained for many scattering channels. In the next step (panel C) the entire REMPI spectrum is fit with predefined rotational state distributions. For this step, signal and background spectra are binned in intervals of $1 \mathrm{~cm}^{-1}$. Then, a corrected REMPI spectrum (panel C, black spectrum) is calculated by multiplying the background spectrum with a factor of 0.8 and subtracting the result from the signal spectrum. The factor of 0.8 accounts for that the dump pulse also reduces fluorescence with a fluorescence depletion of approximately $20 \%$. The fit assumes similar rotational state distributions for all scattered vibrational states belonging to one incidence translational energy and orientation.

As a result of the fit, we obtain the relative band intensities of all $\gamma\left(v^{\prime}, v^{\prime \prime}\right)$-bands in the probed wavelength range, which are the only variable fitting parameters (panel D). The different symbols in the figure describe which vibrational state of $\mathrm{A}^{2} \Sigma^{+}$is used for ionization. Most vibrational states are probed via different $\gamma$-bands.

In the next step (panel E), we select always the same set of $\gamma$-bands for the final averaging of the data. This set of data was selected according to the criteria that 1) the $\gamma$-bands have strong Franck-Condon factors, 2) a large fraction of the band falls within the detected wavelength range, and 3) the bands are not too strongly overlapped with other REMPI bands. The determination of vibrational state distributions with this set of REMPI bands has been tested in a control experiment (140). In this experiment, we

\footnotetext{
${ }^{1}$ We first assumed very high values $J_{\max }$ and later tightened this constraint in case no population at high $J$ values was observed. This way more lines are treated as non-overlapping and we obtain more data points in the rotational state distributions.
} 
generated a known vibrational state distribution via Franck-Condon pumping, meaning that we optically pumped the $\mathrm{B}^{2} \Pi_{1 / 2}\left(v^{\prime}=3, J=0.5\right)$ state of NO with one of the home-built OPO laser systems. The $\mathrm{B}^{2} \Pi_{1 / 2}\left(v^{\prime}=3, J=0.5\right)$ state then fluoresces to the electronic ground state and creates a vibrational state distribution according to the emission coefficients of the $\mathrm{X}^{2} \Pi_{\Omega}\left(v^{\prime \prime}\right) \leftarrow \mathrm{B}^{2} \Pi_{1 / 2}\left(v^{\prime}=3, J=0.5\right)$ bands. We then probed population in $\mathrm{X}^{2} \Pi\left(v^{\prime \prime}\right)$ (which is generated in the rotational states $J=0.5$ and 1.5 due to the selection rule $\Delta J= \pm 1)$ via the $\gamma\left(v^{\prime}, v^{\prime \prime}\right)-$ REMPI bands. We managed to obtain good agreement between calculated and measured vibrational state distributions without correcting the REMPI signal for Franck-Condon factors. This corresponds to the assumption of saturating the first step of the $(1+1)$ REMPI scheme and having the same ionization cross sections for the different selected bands.

In the last step of the analysis (panel F), we average the band intensities of the selected bands. Furthermore, we apply a density-to-flux correction based on ro-vibrational state dependent velocity distributions which have recently been measured in our laboratory by Bastian Krüger $1^{1}$ The effect of the correction to relative population of a final vibrational state is typically smaller than $20 \%$.

\subsubsection{Incidence translational energy dependence of the vibrational relaxation}

Fig. 6.12 shows vibrational state distributions of $\mathrm{NO}$ after scattering from $\mathrm{Au}(111)$ with an isotropic incidence angular orientation distribution, for two different incidence vibrational states $\left(v_{\mathrm{i}}=16\right.$ and 11) and at five different incidence translational energies. There are several important trends in the vibrational state distributions:

1. The vibrational state distributions both for $v_{\mathrm{i}}=11$ and 16 are remarkably similar in a sense, that they peak at around $v=5-6$, largely independent of the incidence translational energy $E_{\text {trans }}^{\mathrm{i}}$.

2. The vibrational relaxation probability for $v_{\mathrm{i}}=11$ (meaning the loss of at least one quantum of vibrational energy) strongly increases with the incidence translational

\footnotetext{
${ }^{1}$ The measurement of speed distributions involved changes to the experimental setup, including a new MCP detector, a translation stage for the REMPI laser and larger windows on the surface chamber to perform optical pumping closer to the surface. We previously (6) published data using a densityto-flux correction based on an extrapolation of data from $v_{\mathrm{i}}=3$ scattering measured by Golibrzuch et al. (10, 149).
} 

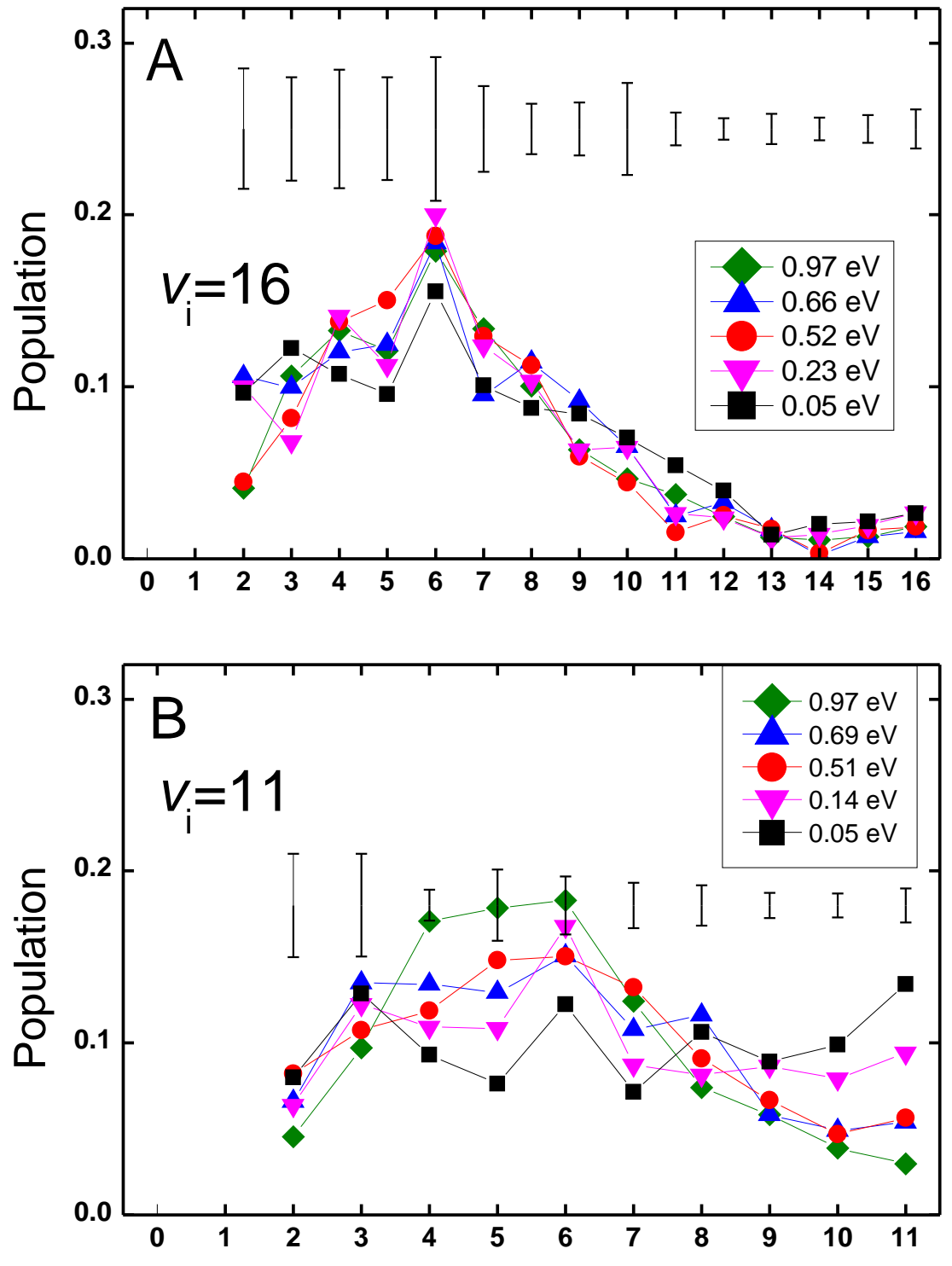

Final vibrational state

Figure 6.12: Vibrational state distributions - Final vibrational state distributions of $\mathrm{NO}$ scattered from $\mathrm{Au}(111)$ with different incidence vibrational states $v_{\mathrm{i}}\left(v_{\mathrm{i}}=16\right.$ in the top panel and $v_{\mathrm{i}}=11$ in the bottom panel) and at different incidence translational energies (given as figure legends). The error bars give an estimate of the error (90\% confidence interval) in each vibrational state. The error bars are calculated from comparing results calculated for different $\gamma\left(v^{\prime}, v^{\prime \prime}\right)$-bands probing the same vibrational state $v^{\prime \prime}$. For $v^{\prime \prime}=2$ in $v_{\mathrm{i}}=11$ and $v^{\prime \prime}=2,3$ in $v_{\mathrm{i}}=16$ only one $\gamma\left(v^{\prime}, v^{\prime \prime}\right)$-band was scanned and the error bars represent an estimated error. 
energy, also see Fig. 6.13. This effect is very similar to the results for the scattering of $\mathrm{NO}\left(v_{\mathrm{i}}=3\right)$.

3. For $v_{\mathrm{i}}=16$, the relaxation probability is almost always near $100 \%$ within the experimental error. For this very high incidence vibrational state, the scattering produces always the same vibrational state distributions, regardless of the incidence translational energy.

Fig. 6.13 shows the relaxation probability as function of the incidence vibrational state and the incidence translational energy. Incidence vibrational as well as translational energy enhance the vibrational relaxation.

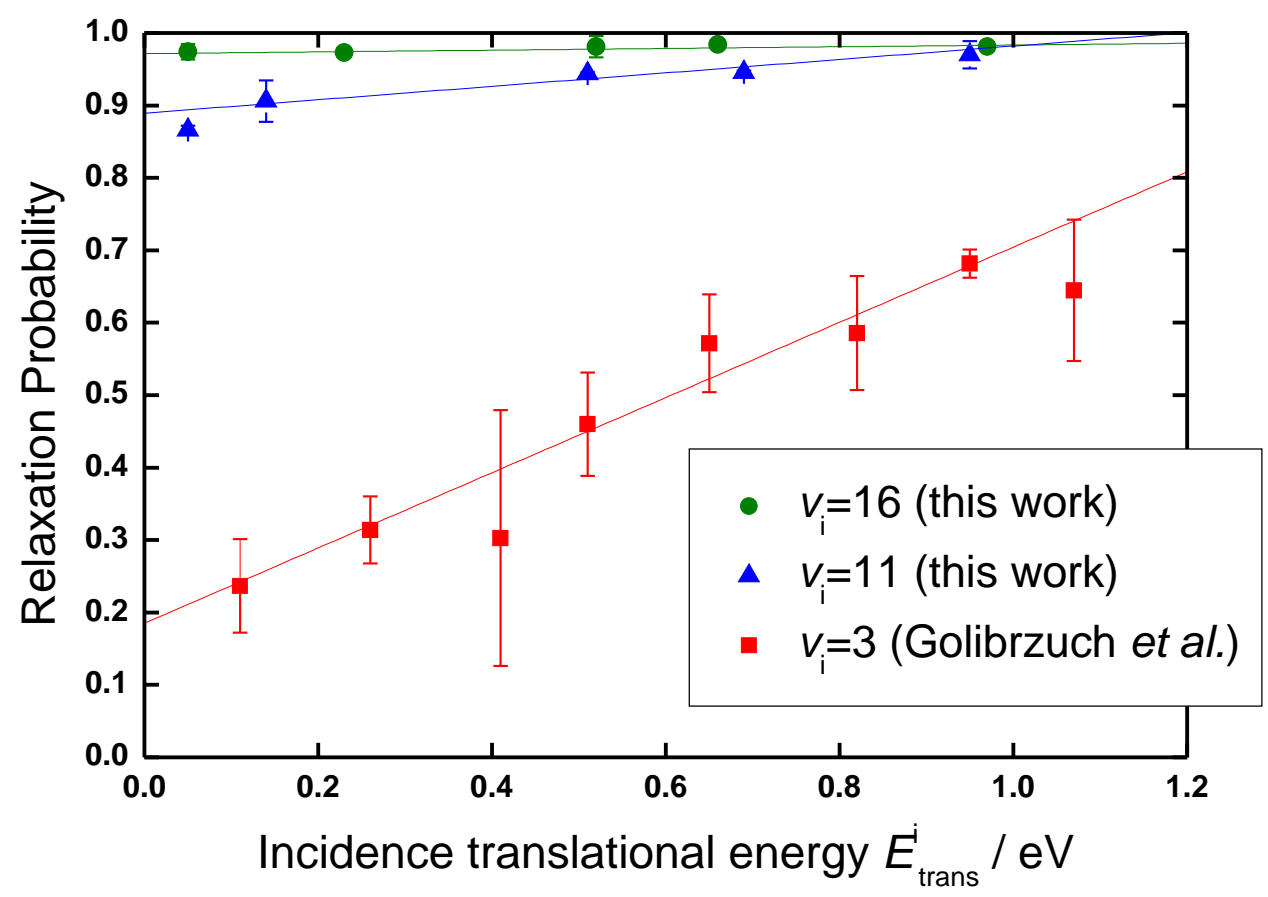

Figure 6.13: Relaxation probability - Probability of loosing at least one quantum of vibrational energy upon collision with $\mathrm{Au}(111)$ for $\mathrm{NO}$ molecules initially prepared in different vibrational states $v_{\mathrm{i}}$ as function of the incidence translational energy $E_{\text {trans }}$. The data for $v_{\mathrm{i}}=3$ is taken from Ref. (10) and has been shown in the right panel of Fig. 2.9 on page 22 of this thesis. The lines are drawn to guide the eye. The calculation of the relaxation probability assumes that no population is transferred to vibrational states that are not detected in the experiment $\left(v=0\right.$ for $v_{\mathrm{i}}=3$ and $v=0,1$ for $\left.v_{\mathrm{i}}=16,11\right)$. 


\subsubsection{Orientation dependence of the vibrational relaxation}

Fig. 6.14 shows how the incidence orientation effects the scattered vibrational state distributions. For $v_{\mathrm{i}}=11$ (Fig. 6.14 B) vibrational relaxation is clearly enhanced by an N-atom first orientation. This is consistent with our previous results for $v_{\mathrm{i}}=3$. Interestingly, this effect disappears completely when molecules are prepared in the very high vibrational state $v_{\mathrm{i}}=16$ (Fig. 6.14 A). In this case, the driving force towards vibrational relaxation becomes that strong, that the incidence orientation - as well as the incidence translational energy — are less crucial.

The inset of Fig. 6.14 B shows the orientation dependent vibrational relaxation probability as function of the incidence translational energy. This data is based on the analysis of short REMPI scans probing only the $\gamma(3,11)$-band around $310 \mathrm{~nm}$ (140) and orientation dependent relaxation probabilites were calculated using the relaxation probabilities for isotropically oriented molecules (Fig. 6.13) as a reference. The effect of the orientation on the relaxation probability clearly decreases with increasing incidence translational energy.

A peculiarity about the data presented in Fig. 6.14 is that the sum of the populations $P(v)$ in the detected vibrational states is not equal for both incidence orientations. Instead, for $v_{\mathrm{i}}=11$ the $\operatorname{sum} \sum_{v=2}^{11} P(v)$ is by a factor of 1.15 higher for molecules oriented with the $\mathrm{O}$-atom towards the surface compared to molecules oriented with the $\mathrm{N}$-atom towards the surface. The same trend is observed for $v_{\mathrm{i}}=16$ and the summed populations $\sum_{v=2}^{16} P(v)$ differ by a factor of 1.20 for the two incidence orientations.$^{1}$ These population differences are too large to be within the experimental error. The data thus suggests, that there is at least a $20 \%$ channel of collision events not detected in the experiment. A possible channel would be bond dissociation. I will discuss this idea in section 6.3.4.

\footnotetext{
${ }^{1}$ Without density-to-flux correction the given factors are even larger $\left(1.17\right.$ for $v_{\mathrm{i}}=11$ and 1.21 for $\left.v_{\mathrm{i}}=16\right)$. Similar factors are obtained by simply integrating the REMPI spectra over the entire scanned wavelength range ( 1.23 for $v_{\mathrm{i}}=11$ and 1.17 for $\left.v_{\mathrm{i}}=16\right)$, although the later treatment is not entirely methodologically sound, because some vibrational states are probed by more $\gamma$-bands than others.
} 

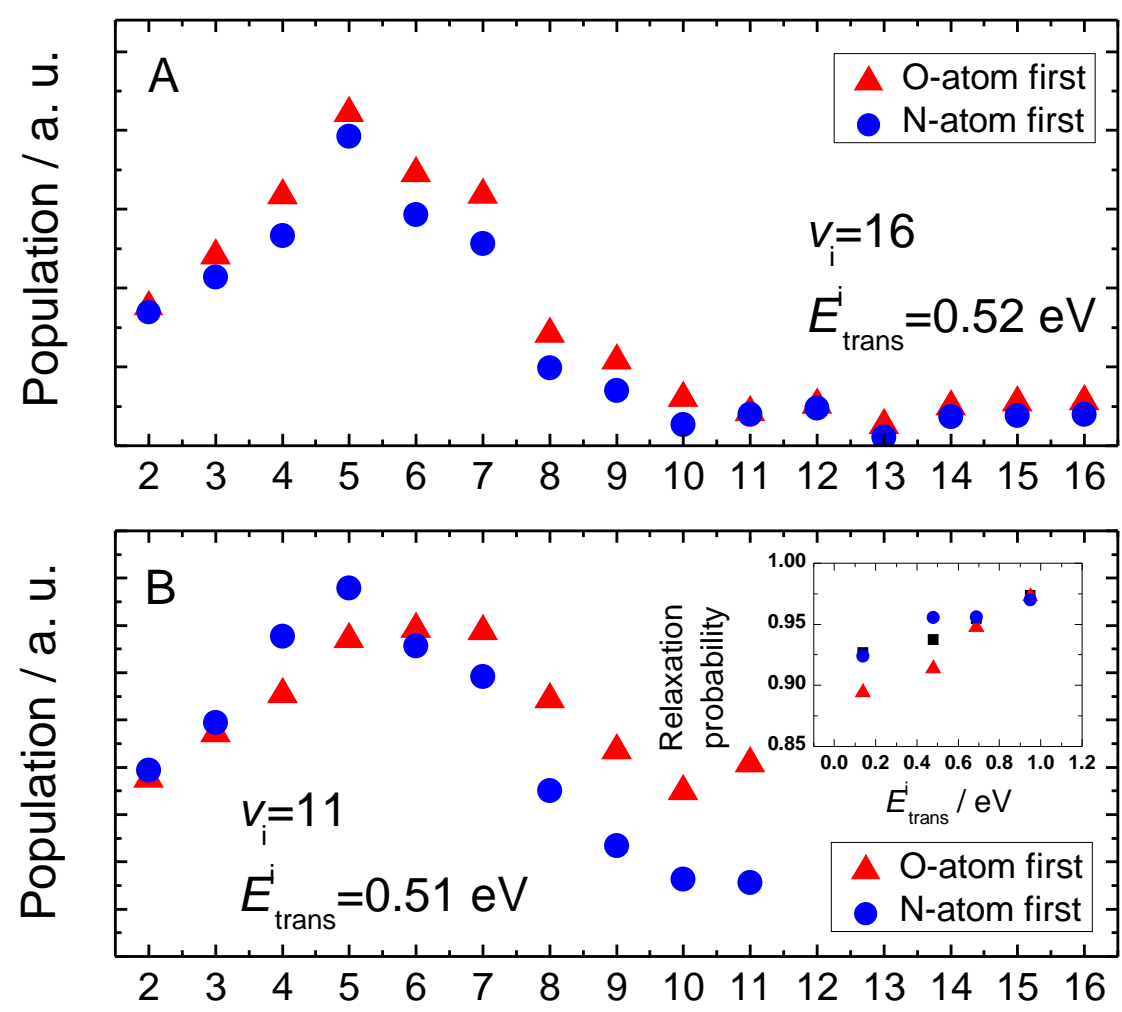

Final vibrational state

Figure 6.14: Orientation dependent vibrational state distributions - Final vibrational state distributions of highly vibrationally excited NO $\left(v_{\mathrm{i}}=16\right.$ and $v_{\mathrm{i}}=11$ in the top and bottom panels, respectively) scattering from $\mathrm{Au}(111)$ as function of the incidence orientation. Molecules are preferentially oriented with the O-atom (red triangles) or the N-atom (blue circles) towards the surface. Population distributions are not normalized. The inset in (B) shows the orientation-dependent relaxation probability as function of the incidence translational energy. The black squares, red triangles, and blue circles indicate isotropic orientation, $\mathrm{O}$-first orientation and $\mathrm{N}$-first orientation, respectively. 


\subsubsection{Rotational state distributions: Rotational rainbow scattering and rotational cooling upon vibrational relaxation}

Fig. 6.15 shows experimental rotational state distributions (and corresponding mean rotational energies) produced by collisions of $\mathrm{NO} \mathrm{X} \mathrm{X}^{2} \Pi_{1 / 2}(v=11, J=0.5)$ with $\mathrm{Au}(111)$ with different incidence translational energies as probed in different scattered vibrational states. This data is based on fitting individual rotational lines in the REMPI spectra. Rotational state distributions shown in the left panel panel are based on an analysis of the $\gamma(3,11), \gamma(1,8)$ and $\gamma(1,6)$ bands for the scattered vibrational states $v=11,8$ and 6 , respectively.

Similar to our observations for $v_{\mathrm{i}}=3$, measured rotational state distributions are highly

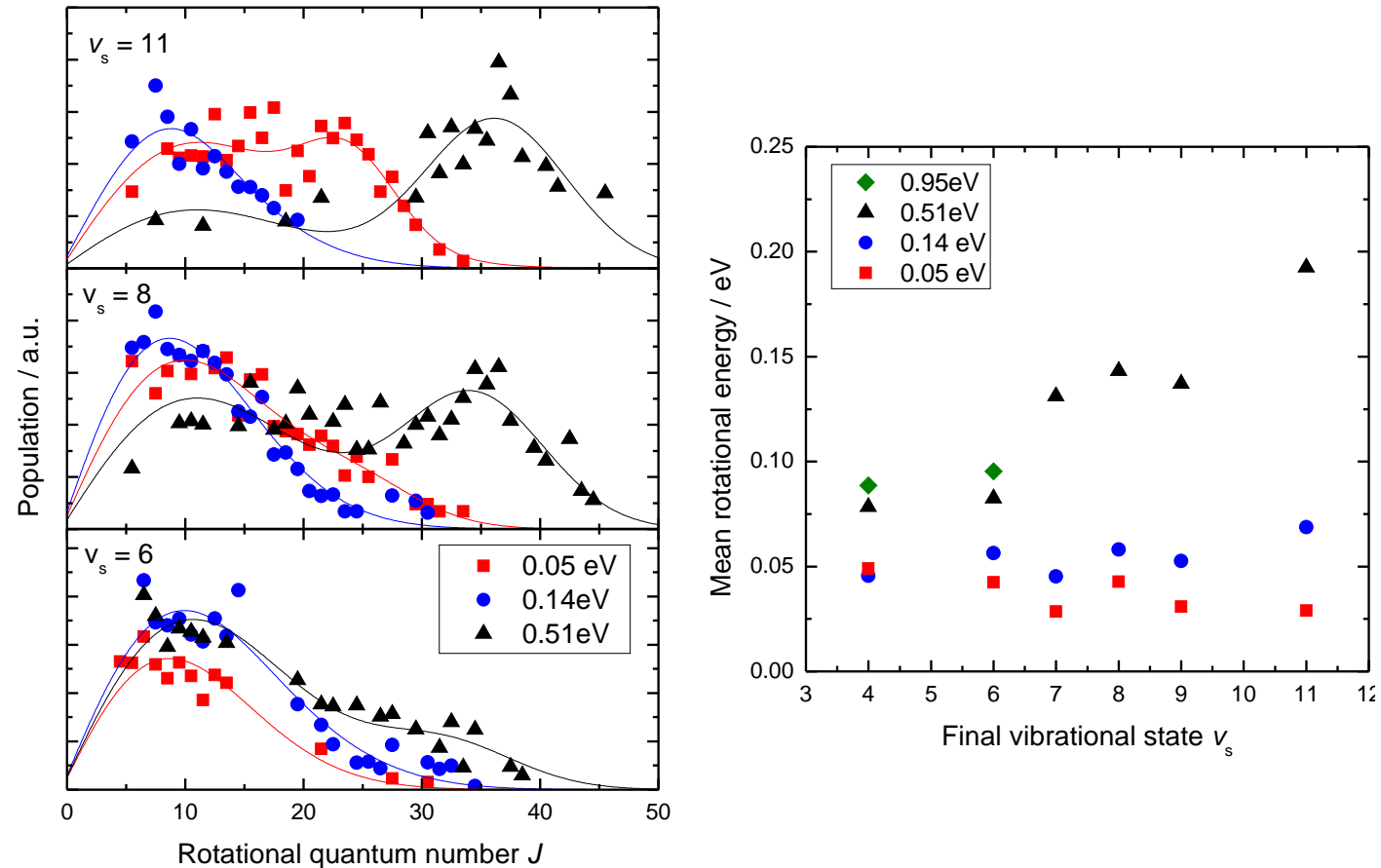

Figure 6.15: Rotational excitation as function of the incidence translational energy and the scattered vibrational state - left: Rotational state distributions generated by collisions of $\mathrm{NO} \mathrm{X}{ }^{2} \Pi_{1 / 2}(v=11, J=0.5)$ with $\mathrm{Au}(111)$ probed in different scattered vibrational states $v=11$ (upper panel), $v=8$ (middle panel), $v=6$ (lower panel) and for three different incidence translational energies $0.51 \mathrm{eV}$ (black triangles), $0.14 \mathrm{eV}$ (blue circles) and $0.05 \mathrm{eV}$ (red squares). The lines are drawn to guide the eye. right: Mean scattered rotational energy as function of the final vibrational state and for different incidence translational energies. 
non-thermal and we observe a pronounced rotational rainbow structure. The $\mathrm{O}$-first rotational rainbow (at high $J$ ) is most strongly pronounced in the vibrational elastic channel (in this case $v=11 \rightarrow 11$ ). It reduces in the $v=11 \rightarrow 8$ channel and is almost non-existent in the $v=11 \rightarrow 6$ scattering channel. In general, the loss of vibrational quanta is accompanied by a decrease of rotational excitation (rotational cooling upon vibrational relaxation). Molecules scattering under conditions where they transfer more vibrational energy to the surface at the same time take up less rotational energy from their incidence translation. Here, the data for the lowest incidence translational energy of $0.04 \mathrm{eV}$ is an exception and the rotational excitation is almost independent of the detected vibrational state.

Fig. 6.16 shows the rotational state distributions when the NO molecules in $\mathrm{X}^{2} \Pi_{1 / 2}(v=$ $11, J=0.5)$ are scattered with an $\mathrm{N}$-atom first or $\mathrm{O}$-atom first incidence angular orientation distribution. The shown data belongs to the measurement with an incidence translational energy of $0.51 \mathrm{eV}$.

In particular for the vibrational elastic channel, the incidence angular distribution has a strong effect of the scattered rotational state distributions. The O-first rotational rainbow in the $v=11 \rightarrow 11$ channel is strongly enhanced by collisions when scattering the NO molecules with the $\mathrm{O}$-atom first. Again, the rotational rainbow structure can also be observed in the vibrationally relaxed channels (e.g. the $v=11 \rightarrow 8$ channel). However, difference in rotational excitation between the initially oriented molecules decreases with increasing loss of vibrational energy to the surface. 

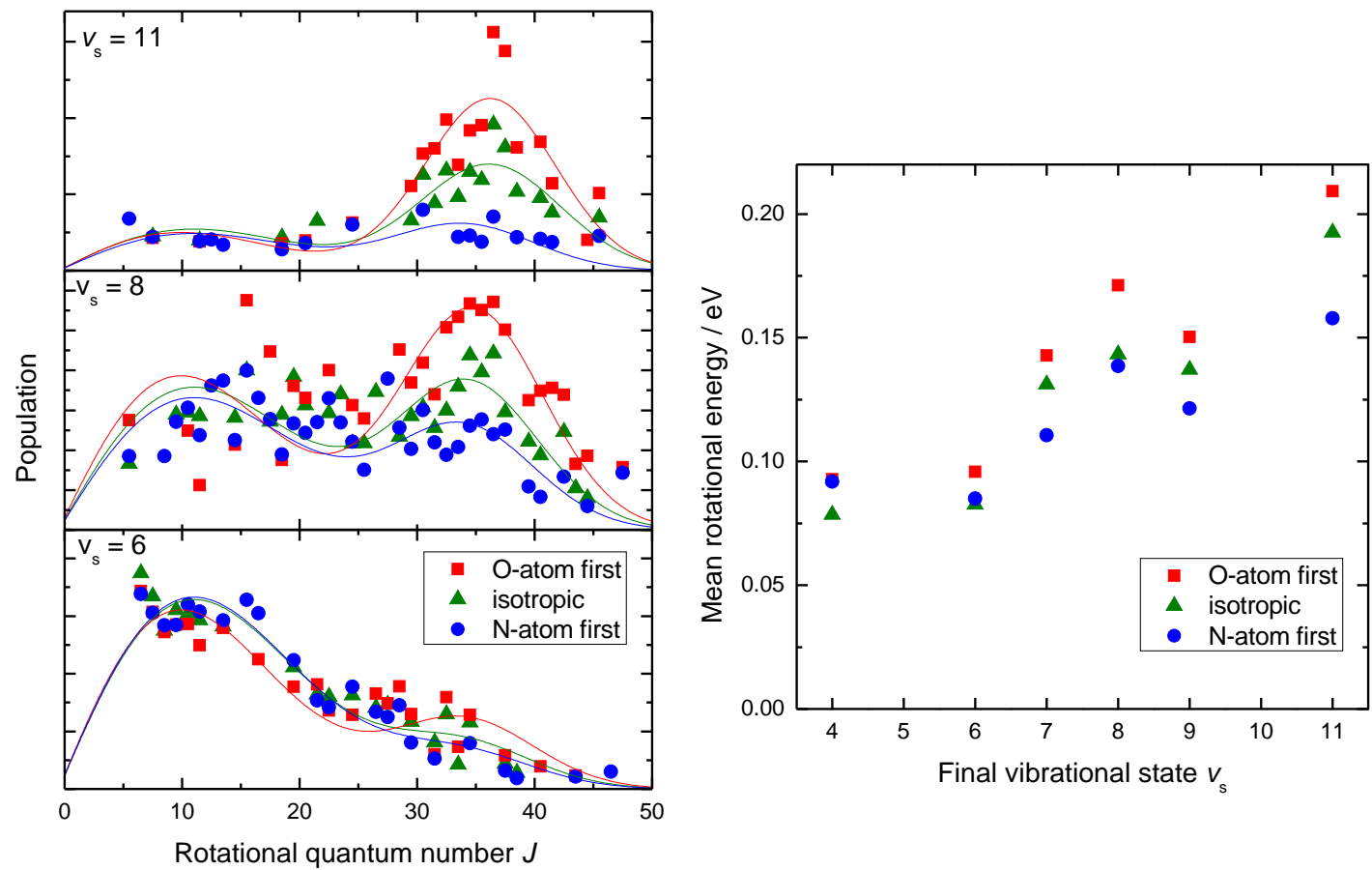

Figure 6.16: Orientation dependence of the rotational state distributions left: Rotational state distributions as influenced by the angular distribution when NO $\mathrm{X}^{2} \Pi_{1 / 2}\left(v_{\mathrm{i}}=11, J=0.5\right)$ scatters from the $\mathrm{Au}(111)$ surface with $0.51 \mathrm{eV}$ incidence translational energy. The different panels (from top to bottom) correspond to the rotational state distributions probed in three different scattered vibrational states $(v=11,8$, and 6$)$. Molecules in the incident molecular beam were preferentially oriented with the $\mathrm{O}$-atom towards the surface (red squares), the $\mathrm{N}$-atom towards the surface (blue circles) or have been scattered with an isotropic incidence angular molecular distribution (green triangles). The lines are drawn to guide the eye. right: Orientation-dependent mean rotational energies as function of the final vibrational state (again for $0.51 \mathrm{eV}$ incidence translational energy). 


\subsection{Discussion}

\subsubsection{Vibrational relaxation in the framework of an electron transfer model}

The results for the vibrational state distributions of NO scattering from $\mathrm{Au}(111)$ unambiguously show, that the vibrational relaxation is enhanced by incidence vibrational as well as translational excitation and $\mathrm{N}$-atom first orientation of the scattering molecule. These trends can be qualitatively understood by analysing semi-empirical model potentials of $\mathrm{NO}$ and $\mathrm{NO}^{-}$species near the surface (see Fig. 6.17) following the work of White et al.(152). The plotted neutral $\mathrm{NO} / \mathrm{Au}(111)$ potential uses parameters developed for the NO-Ag(111) interaction and is modified to fit the position of the potential energy well for NO approaching a $\mathrm{Au}(111)$ surface at an hexagonal close-packed hollow site (1.82 $\AA$, reported in Ref. (67)). Details on the potentials can be found in the supplementary material of Ref. (6). The interaction of the anionic species with the surface is approximated by the image charge effect (equation 2.12 on page 15 ). The energetically most advantageous bond length for electron transfer is at the outer classical turning point of the vibration.(2) These bond seperations are thus used in the panels of Fig. 6.17. This treatment of vibrationally excited molecules as stretched molecules(117) is justified by the high probability density near the classical outer turning point. The anionic potential is strongly affected by the orientation, since the lowest unoccupied molecular orbital (LUMO) of NO has a higher probability density on the N-atom than on the $\mathrm{O}$-atom (according to density functional theory, $30 \%$ of the electronic charge is located an the $\mathrm{O}-$ atom (153) $)$.

This simple analysis suggests, that there is an energetic barrier towards electron transfer, when the NO molecule approaches the surface. See Fig. 6.17. Within this model, the barrier towards ET ranges from $0.7 \mathrm{eV}$ to $0.1 \mathrm{eV}$ between $v_{\mathrm{i}}=3$ and $v_{\mathrm{i}}=16$ and is always higher for $\mathrm{O}$-atom first orientation than for $\mathrm{N}$-atom first orientation. It does not only explain the observations that incidence vibrational energy and $\mathrm{N}$-atom first orientation (at least for $v_{\mathrm{i}}=3$ and $v_{\mathrm{i}}=11$ ) promote the electron-transfer driven vibrational relaxation, but also the enhancement at increased incidence translational energy. The incidence translational energy helps in ascending the barrier towards ET. Independently of this energetic argumentation, the enhancement of the vibrational relaxation by $\mathrm{N}$-atom first collisions is also supported by the steric argument, that 


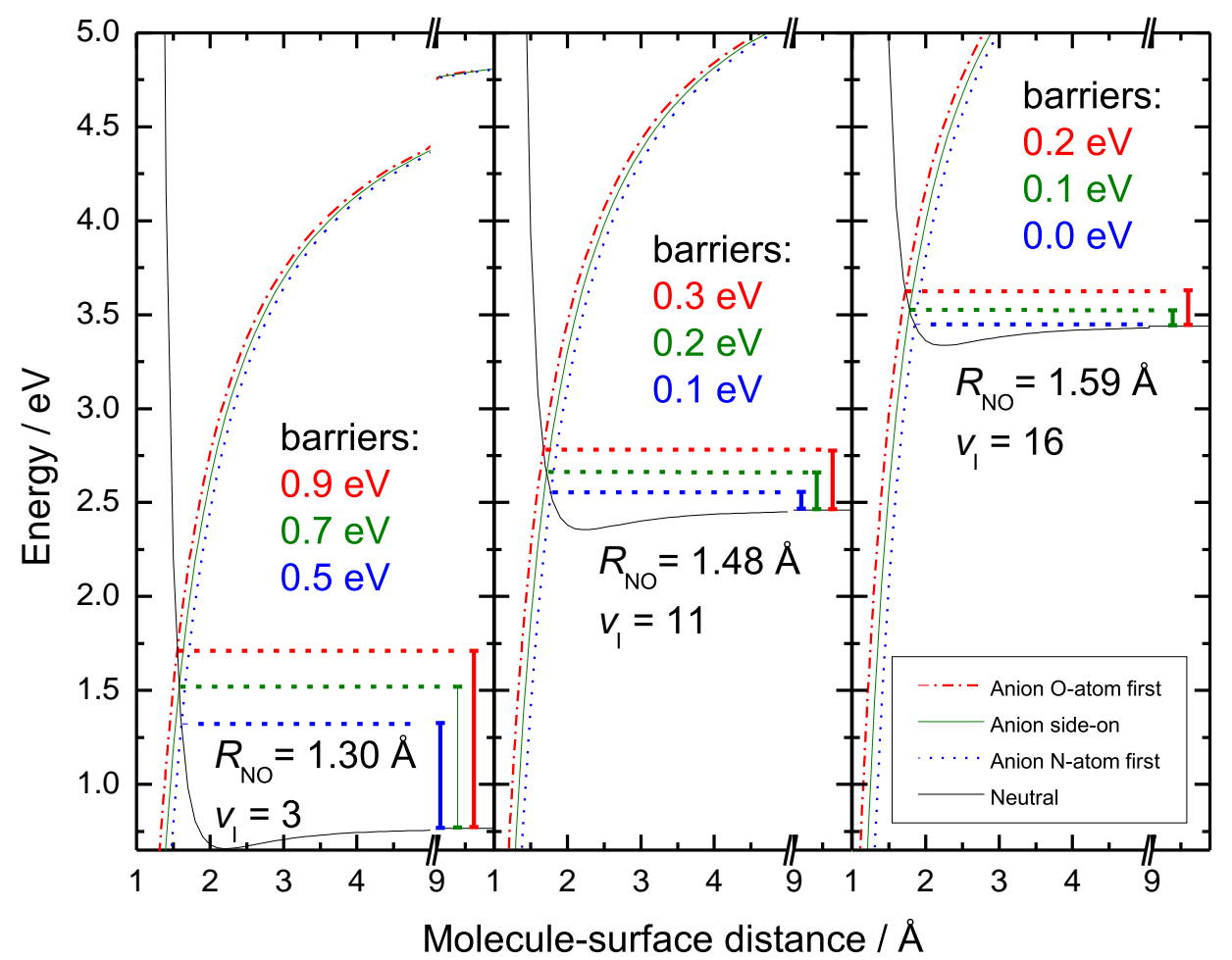

Figure 6.17: Vibrational relaxation in the framework of an electron transfer model - The figure shows semi-empirical model potentials for $\mathrm{NO}^{-}$and $\mathrm{NO}^{-}$species in the vicinity of the metal surface. The different panels describe the energetics with NO bonds length restricted to the outer classical turning points of the molecule's vibration in the incidence vibrational states $v_{\mathrm{i}}=3$ (left panel), $v_{\mathrm{i}}=11$ (middle panel) and $v_{\mathrm{i}}=16$ (right panel). The curves are constructed following the lines of White et al. (152). The anionic potentials are strongly orientation dependent and are calculated for orientation angles of $68^{\circ}$ (expectation value $\langle\theta\rangle$ for $\mathrm{O}$-atom first orientation, red dash-dotted line), $90^{\circ}$ (side-on orientation, green solid line) and $68^{\circ}(\langle\theta\rangle$ for $\mathrm{O}$-atom first orientation, blue dotted line). In principle, the neutral potential (black line) is also orientation dependent, which is however much weaker than for the ionic curves and has thus been omitted in the figure. Figure from Ref. (6). 
electron transfer requires an orbital overlap between occupied orbitals in the metal (or occupied electronic bands in the terms of solid state physics) and the $\mathrm{N}$-atom centered $\pi^{*}$-LUMO of the NO molecule. The observation that vibrational state distributions among the relaxed vibrational states for the scattering of $v_{\mathrm{i}}=16$ and 11 might indicate, that the collision always generates the same vibrational state distributions once the barrier towards ET has been crossed. The observation that vibrational relaxation is not affected by the molecules orientation and incidence translational energy for $v_{\mathrm{i}}=16$ and the relaxation probability approaches $100 \%$ can be attributed to the vanishing of the barrier for this very high vibrational state. This means, that there is a complex interplay between the factors of the incidence vibrational energy, translational energy and orientation that all affect the probability of the transient electron transfer that underlies the vibrational relaxation.

One of the referees of our publication (6) argued, that the electron can also tunnel through the barrier and that the barrier width would affect the tunnelling probability. However, the width of barrier is also smallest near the curve crossing and the trends for tunnelling are qualitatively expected to be similar to those for crossing the barrier classically.

\subsubsection{Rotational rainbows and the origin of rotational cooling}

A particularly interesting finding in the scattered rotational state distributions is that molecules loosing vibrational energy during the collision generally have less rotational excitation than molecules scattered without vibrational energy loss. This rotational cooling upon vibrational relaxation is highly counter-intuitive, because one might expect that the collision leads to an energy transfer from incidence vibration to scattered rotation. I will show that the observed rotational state distributions can be rationalized with classical molecular dynamics (MD) simulations, if it is assumed that the rotational excitation and the vibrational relaxation are - at least to a first approximation - energetically decoupled. Instead, the vibrational relaxation only acts as filter to the rotational state distributions.

Fig. 6.18 shows results from MD simulations treating the NO molecule as a rigid rotor on the empirical orientation dependent potential $V(z, \theta)$ from Ref. (87). This strongly asymmetric potential — which was developed by fitting experimental rotational state distributions for the (orientation dependent) scattering of $\mathrm{NO}(v=0 \rightarrow 0)$ 
from $\operatorname{Ag}(111)$ - has the functional form

$$
\begin{aligned}
V(z, \theta) / \mathrm{eV}= & \frac{10}{\left(\left(z / \mathrm{a}_{0}\right)+0.16 \cos \theta+0.16 \cos ^{2} \theta\right)^{8}} \\
& -\frac{1}{\left(\left(z / \mathrm{a}_{0}\right)+0.2 \cos \theta\right)^{3}}-\exp \left[-\left(\frac{4}{\left(z / \mathrm{a}_{0}\right)}-1\right)^{2}\right] \frac{5}{\left(z / \mathrm{a}_{0}\right)^{4}},
\end{aligned}
$$

where $\mathrm{a}_{0}$ is the Bohr radius. A contour plot of the potential is shown in Fig. 2.17 (page 36 of this thesis).

Panel A of Fig. 6.18 shows the rotational angular momentum for five different incidence translational energies (I chose the energies used for the $v_{\mathrm{i}}=3$ scattering experiments) as function of the orientation angle $\theta$. Each angular momentum corresponds to a specific rotational energy which is given in panel B. At angles $\theta$ of $0^{\circ}$ and $180^{\circ}$ (corresponding to $\mathrm{O}$-first and $\mathrm{N}$-first end-on orientations) no angular momentum and thus no rotational excitation is generated by the collisions with the surface. Instead, there are two extrema at $\theta \approx 48^{\circ}$ and $\theta \approx 144^{\circ}$ where most incidence translation is converted into scattered rotation. The exact rotational state distributions predicted by the simulation depend on the combination of 1) the rotational energy as function of $\theta$ (panel B) and 2) the incidence angular distribution in the experiment. The incidence angular distributions for isotropic as well as pre-oriented (the shown angular distributions for oriented molecules correspond to the high-field limit) molecules have been calculated earlier in this thesis (section 4) and are given in panel C. Panel D of Fig. 6.18 shows the calculated rotational state distribution, in case a molecular beam with isotropic incidence angular distribution scatters from the surface. The rotational state distributions have a component dominated by collisions with incident angles $>90^{\circ}(J \approx 0-12$, the $\mathrm{N}$-first rotational rainbow) and a second component mostly generated from collisions with incident angles $>90^{\circ}$ (high $J$, O-first rotational rainbow). Both rotational rainbows shift to higher $J$ with increasing incidence translational energy, although this effect is more pronounced for the $\mathrm{O}-$-first rotational rainbow.

Up to this point in the discussion, I basically reviewed how rotational state distributions were explained in 1991 and the new question is what happens in case molecules change their vibrational state. The simplest assumption is that the vibrational relaxation (in which energy is transferred from the vibration of the molecule to electronic degrees of freedom in the metal) is an effect completely decoupled from the rotational excitation 

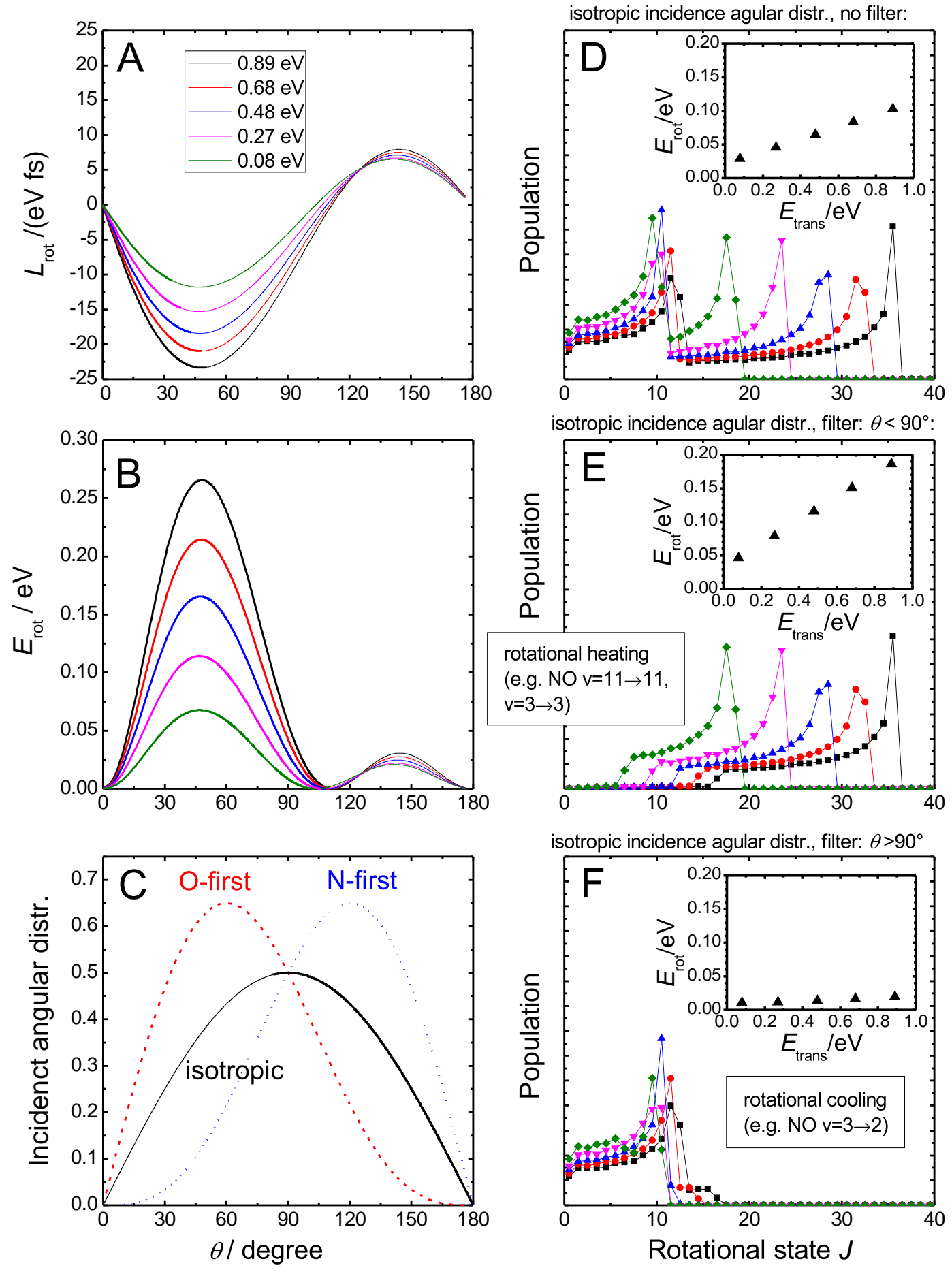

Figure 6.18: Explanation of the rotational rainbows and rotational cooling Classical molecular dynamics simulations using the VSW-potential $V(z, \theta)$ developed for the scattering of $\mathrm{NO}$ from $\mathrm{Ag}(111)$. A filtering effect for the orientation angle $\theta$ can explain rotational cooling upon vibrational relaxation. The different panels are described in the text. 
(in which translational energy of the molecule is converted to scattered rotational energy). Both effects however depend strongly on the incidence orientation. This means, that the vibrational relaxation could act as an angular filter to the rotational state distributions.

As a simple example, panels $\mathrm{E}$ and $\mathrm{F}$ of Fig. 6.18 show rotational state distributions generated assuming that all molecules with an incidence angle $\theta$ in a range of $\theta=0^{\circ} . .90^{\circ}$ scatter from the surface without vibrational relaxation (panel E) and all other molecules $\left(\theta=90^{\circ} . .180^{\circ}\right)$ relax to a lower vibrational state (panel E). In this case, the scattering experiment is expected to generate a very hot rotational state distribution in the vibrationally elastic channel and a very cold rotational state distribution in the vibrational inelastic channel. The later of these effects corresponds to the rotational cooling upon vibrational relaxation and the first effect could in analogy be labelled rotational heating upon vibrational non-relaxation. This concept is also described in Fig. 6.19.

The predictions of this simple model agree well with our experimental observations. For
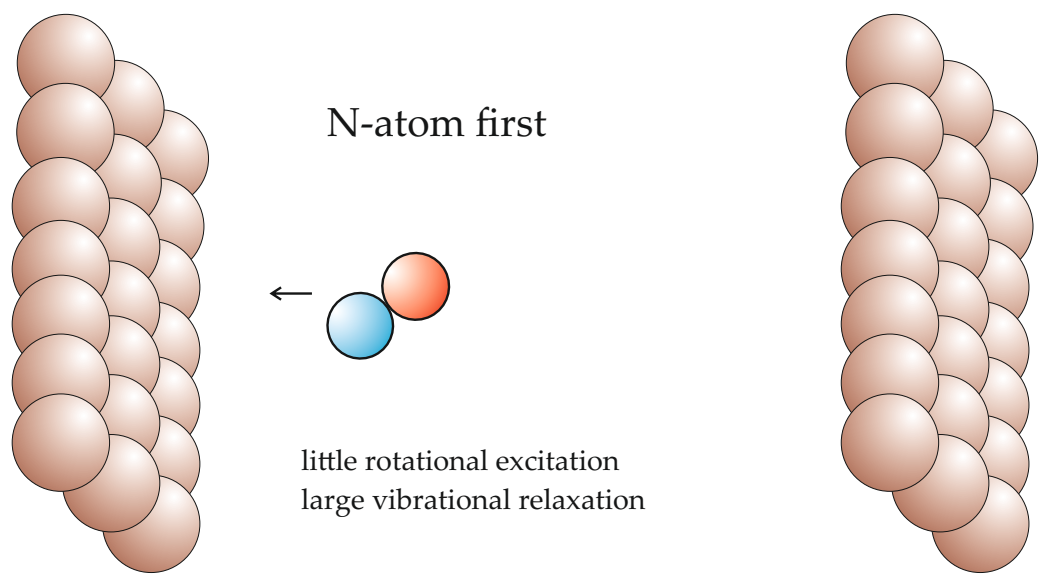

O-atom first

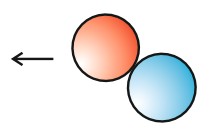

large rotational excitation little vibrational relaxation

Figure 6.19: Rotational cooling in vibrational relaxation of NO scattering from Au(111). - N-atom first collisions lead to more effective vibrational relaxation but less rotational excitation. In contrast, $\mathrm{O}$-atom first collisions lead to more rotational excitation but less probable vibrational relaxation.

$v_{\mathrm{i}}=3$, the rotational state distributions in $v_{\mathrm{s}}=3$ show pronounced $\mathrm{O}-$-first rotational rainbows, as this state has enhanced population from O-first collisions. As opposed to this, molecules scattered in the $v=3 \rightarrow 2$ channel have small rotational excitation as this channel is almost exclusively populated collisions with $\mathrm{N}$-atom first orientation. A very similar argumentation can be used to explain the rotational state distributions for 
$v_{\mathrm{i}}=11$ (see Fig. 6.15 and 6.16). Here, $\mathrm{N}$-atom first collisions again favour strong vibrational relaxation and small rotational excitation. When it is assumed, that the loss of an increasing number of vibrational states $\Delta v$ during the collisions imposes stronger constraints to the angular orientation distributions of relaxing molecules, this concept can also explain, that the mean rotational energy decreases with increasing $\Delta v$.

\subsubsection{Dynamical steering at low translational energy}

An important question in gas-surface (as well as gas phase) reaction dynamics is whether a molecule that approaches a surface (another molecule) in a certain specific geometry is reoriented to a different geometry due to long-range interaction forces. This effect is known as dynamical steering and can have strong impact on chemical reactivity.

As an example from the gas phase, it was observed that the $\mathrm{Cl}+\mathrm{HD}$ reaction strongly favors $\mathrm{DCl}$ formation over $\mathrm{HCl}$ formation. This result could only be reproduced by quantum mechanical calculations, in case steering effects due to van der Waals interactions were taken into account. The calculations suggest that in case the $\mathrm{Cl}$ atom approaches the $\mathrm{D}$ atom first, the reaction predominantly generates the $\mathrm{DCl}+\mathrm{H}$ product. However, when the $\mathrm{Cl}$ atom approaches the $\mathrm{H}$ atom first, van der Waals interactions steer the system towards perpendicular geometries, which are less reactive.(154) In gas-surface dynamics, dynamcial steering is more difficult to investigate due to the multi-dimensionality of the systems. Nevertheless, dynamical steering effects are blieved to be important. For example, the enhanced dissociation probability of $\mathrm{H}_{2}$ at $\mathrm{Pd}(100)$ for an incidence translational energy of $0.02 \mathrm{eV}$ compared to the probability at $0.25 \mathrm{eV}$, has been explained by a dynamical steering effect at the lower incidence translation, which steers molecules approaching a surface over an on-top site to a hollow site. At the higher incidence translation, the interacation time is too short for this steering to occur and the reactivity decreases.(111, 155, 156)

A different type of dynamical steering would be, intermolecular forces steering an approachung molecule to a different molecular orientation with respect to the surface (described by the orientation angle $\theta$ ). Indeed, there are indications, that (at least) this type of dynamical steering occurs at low incidence translational energy in the experiment described in this thesis. 
Fig. 6.7 shows that the asymmetry parameter in the $v=3 \rightarrow 2$ and $v=3 \rightarrow 1$ channels is constant over the range of incidence translational energies between $0.27 \mathrm{eV}$ and $0.89 \mathrm{eV}$, but drops significantly for the lowest incidence energy of $0.08 \mathrm{eV}$. This result is very suprising, because there is no reason to believe that the electron transfer driven vibrational relaxation becomes independent of the incidence molecular orientation at the lowest incidence translation. Another interesting observation at this lowest incidence translational energy is that the rotational temperature (Fig. 6.6) as well as the relative populations of the different spin-orbit states (Fig. 6.8) become independent of the molecular orientation. Furthermore, there is hardly any $\mathrm{O}$-first rotational rainbow that can be observed in the rotational state distributions of the $v=3 \rightarrow 3$ channel at this translational energy (panel (e) of Fig. 6.5) 1. These observations point towards a picture, in which the incident orientation determined by the electric Stark field (which is weak compared to any inter-molecular forces) are diminished. This is likely to be the result of a dynamical steering process.

Of course, at very low incidence translational energy, molecules also have a probability to undergo trapping followed by desorption. Experiments from Wodtke et al. for NO scattering from $\mathrm{Au}(111)$ in $v_{\mathrm{i}}=2$ show that trapping is unimportant above $0.3 \mathrm{eV}$, but is significant at lower incidence translational energy $(0.1-0.26 \mathrm{eV}) .(157)$ The fate of these trapped molecules, e.g. their probabilities of loosing vibrational energy to the surface is not yet well understood. The trapping channel is thus a dark channel that could also be orientation dependent and might effect the detected vibrational state distributions in a manner that is difficult to predict.

\subsubsection{The possibility of NO bond dissociation}

Another important question is whether the scattering of highly vibrationally excited NO from $\mathrm{Au}(111)$ involves a reaction channel in which NO molecules dissociate. Even in cases, where molecules only approach the barrier towards disscociation, this might be important to the reaction dynamics. For the interaction of $\mathrm{H}_{2}$ at $\mathrm{Cu}(111)$ it has been

\footnotetext{
${ }^{1}$ The maximum of this $\mathrm{O}$-first rotational rainbow is predicted to be at around $J=18.5$ and the Nfirst rotational rainbow at $J \approx 10.5$ according to the classical MD simulations. The broadening of the rotational rainbow structure, e.g. due to quantum effects, thermal motion of the surface atoms, surface corrugation and the velocity spread in the incident molecular beam $(\Delta E / E \approx 15 \%)$ might make it difficult to clearly distinguish these roational rainbows. Nevertheless, the rotational state distributions should be different for the opposite incidence orientations.
} 
shown, that vibrational energy transfer can be enhanced by trajectories that traverse but do not cross the transition state towards dissociation.(158)

As stated in section 6.2.5, a peculiarity of the experimental data for the scattering of oriented, highly vibrationally excited NO was, that the sum of the detected populations between $v=2$ and $v=11$ (as well as the sum between $v=2$ and $v=16$ for $v_{\mathrm{i}}=16$ ) did not match for the different orientations. The extrapolation of the vibrational state distributions to $v=0$ as well as theoretical work (66) indicate, that the states $v=0$ and $v=1$ have rather small populations. Furthermore, the orientation experiments were performed at translational energies $(0.5 \mathrm{eV})$ where trapping is thought to be unimportant.(157) This suggests, that the population mismatch might be the result of a reaction channel in which NO molecules dissociate upon the collision. In this regard, it is interesting that a recent DFT study (159) reported a calculated dissociation barrier of $\mathrm{NO}$ on $\mathrm{Au}(111)$ of $3.4 \mathrm{eV}$. An NO molecule in $v_{\mathrm{i}}=16$ has a vibrational energy of $3.3 \mathrm{eV}\left(2.4 \mathrm{eV}\right.$ for $\left.v_{\mathrm{i}}=11\right)$. Adding the translational energy of $0.5 \mathrm{eV}$ yields a total energy of $3.8 \mathrm{eV}\left(2.9 \mathrm{eV}\right.$ for $\left.v_{\mathrm{i}}=11\right)$, which is comparable to the calculated dissociation energy.

So far, we have not managed to directly probe NO bond dissociation. Dissociation is difficult to detect with Auger electron spectroscopy, because the relevant species have very high desorption rates even at liquid nitrogen temperature $\left(\mathrm{N}_{2}\right.$ on $\mathrm{Au}(111)$ desorbs at circa $40 \mathrm{~K}$ in a temperature programmed desorption experiment (160)). We also tried to find an $\mathrm{N}_{2}(2+1)$ REMPI signal by ionizing via $\mathrm{a}^{\prime \prime 1} \Sigma_{g}^{+} \leftarrow \mathrm{X}^{1} \Sigma_{g}^{+}$(161) at 202$203 \mathrm{~nm}$. Unfortunately, this REMPI signal is superposed by a strong background from non-resonant $\mathrm{NO}$ ionization. In principle, $\mathrm{NO}^{+}$and $\mathrm{N}_{2}^{+}$ions can be separated in the ion detection due to their different mass to charge ratio. The time-of-flight resolution in the experiment was however insufficient for signal separation.

\subsubsection{A brief comparison to theoretical models}

\subsubsection{Independent electron surface hopping (IESH) model from the Tully group}

The computer code for the calculations from Shenvi et al. (see section 2.4.3), which were able to reproduce the vibrational state distribution for $\mathrm{NO}^{2} \Pi_{1 / 2}(v=15)$ scattering from $\mathrm{Au}(111)$ at a translational energy of $0.05 \mathrm{eV}$ (the Huang experiment) is 
available to our group. Calculations published by Shenvi et al. (2) used the independent electron surface hopping (IESH) algorithm to calculate trajectories and to account for electronically non-adiabatic effects. However, the code also includes implementations for molecular dynamics with electronic friction (MDEF) and adiabatic molecular dynamics simulations and all three methods use the same PES(s). We recently published (7) a comparison of our experimental vibrational state distribtions to calculations with these methods.

Even prior to this new publication, some problems with the model were apparent. In particular, Golibrzuch et al. have shown, that calculations on the PES from Shenvi et $a l$. predict a high number of multi-bounce collisions even at elevated incidence translational energies.(56) This is in disagreement with experimental observations, in particular at high scattered translational energies(10), where molecules scatter with narrow angular distributions that peak at specular scattering (10). In addition, - with regard to this work - also the observation of pronounced rotational rainbow structure in the rotational state distributions cannot be explained by multi-bounce events. Golibrzuch et al. thus called the high number of multi-bounce collisions in the calculations a "multi-bounce artefact" and suggested, that it results from "subtle errors in the adiabatic interaction potential". They furthermore showed that an improved agreement between experimental and theoretical results can be obtained by simply filtering only the single-bounce collisions from the theoretical calculations (provisional multi-bounce correction).(56)

Fig. 6.20 compares experimental vibrational state distributions at two different incidence translational energies to calculations using IESH and MDEF algorithms, respectively. Results are given with and without applying the provisional multi-bounce correction. It is found that both calculations strongly underestimate the effectiveness of vibrational relaxation, regardless of whether all trajectories are included in the evaluation or only single-bounce trajectories are evaluated. At high incidence translational energy, the number of multi-bounce collisions in the IESH calculations is small anyhow (at $E_{\text {trans }}^{\mathrm{i}}=1 \mathrm{eV}$, approximately $2 / 3$ of the trajectories exhibit single-bounce collisions and most other trajectories exhibit double-bounce collisions). Furthermore, the calculated probability of vibrational relaxation decreases with increasing incidence translation. This is the opposite trend observed in the experiment. In this light, the 


\section{Translational energy}

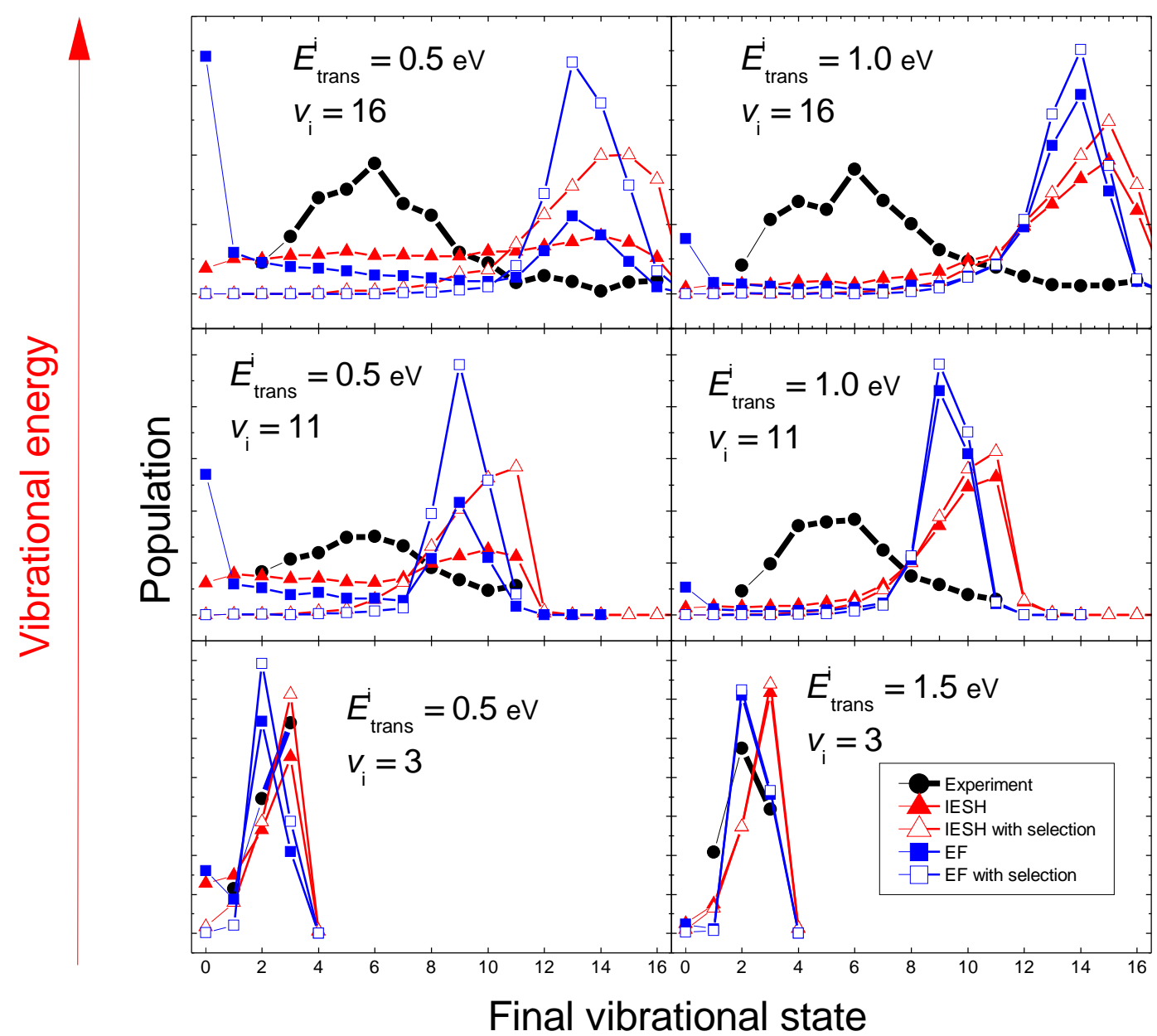

Figure 6.20: Comparison of experimental vibrational state distributions to theoretical calculations - IESH and MDEF calculations underestimate the effectivness of vibrational relaxation for higher vibrational states $\left(v_{\mathrm{i}}=11\right.$ and $\left.v_{\mathrm{i}}=16\right)$ at elevated incidence translational energy. Open symbols indicate calculated vibrational state distributions in case only single-bounce trajectories are filtered from the calculation. Calculations have been performed by C. Bartels. Adapted with permission from (7). Copyright 2015 American Chemical Society. 


\section{Vibrationally inelastic scattering of oriented NO from $\mathrm{Au}(111)$}

agreement between experiment and theory at low incidence translational energy appears coincidental.

The prediction of Shenvi et al.(2) that - according to the IESH calculations - the vibrational relaxation should be strongly dependent on the molecule's orientation was part of the motivation to investigate this effect experimentally. Fig.6.21 compares IESH calculations for oriented molecules to experimental data for the scattering of $\mathrm{NO}(v=3)$ at high translational energy, where strong orientation effects have been observed in the experiment. The left panels $(\mathrm{A}-\mathrm{C})$ show the vibrational state distributions. Panel A shows the results of an IESH calculation performed with starting geometries where orientation angles in the range of $0 . .30^{\circ}$ (O-first orientation) and $150 . .180^{\circ}$ (N-first orientation) were selected from a superset of randomly oriented molecules. In this case, the calculated results agree with the experimental data (panel $\mathrm{C}$ ) in that the vibrational relaxation is strongly enhanced by an $\mathrm{N}$-atom first orientation. However, in panel B) the correct incidence angular distributions as used in the experiment were chosen (compare Fig. 4.8 on page 65). In this case, the orientation dependence of the vibrational relaxation is almost entirely lost. This means, that the IESH calculations qualitatively predict the correct orientation dependence of the relaxation rates, but do not agree with the experimental data under more realistic scattering conditions.

A similar behaviour is found when comparing experimental and calculated rotational state distributions. Calculations with orientation angles in the range of $0 . .30^{\circ}$ and $150 . .180^{\circ}$ ( $\mathrm{N}$-first orientation) (panel $\mathrm{D}$ ) give the same qualitative trends as the experiment (panel F): O-first collisions produce much higher rotational excitation than $\mathrm{N}$-first collisions. The effect is however completely lost, when correct incidence angular distributions are used for the calculations.

In summary, the comparison between the IESH theory and the experiment clearly show severe disagreement regarding vibrational as well as rotational state distributions. What are possible reasons for this disagreement? As discussed in Ref. (7), the computational approach consists of two major parts: 1) the construction of the PES (or PESs) and 2) the (non-adiabatic) propagation.

The comparison of computational and experimental results suggest, that there are major problems with the PES. Calculations on the currently implemented PES produce the multi-bounce artefact independently of whether the propagation is performed 

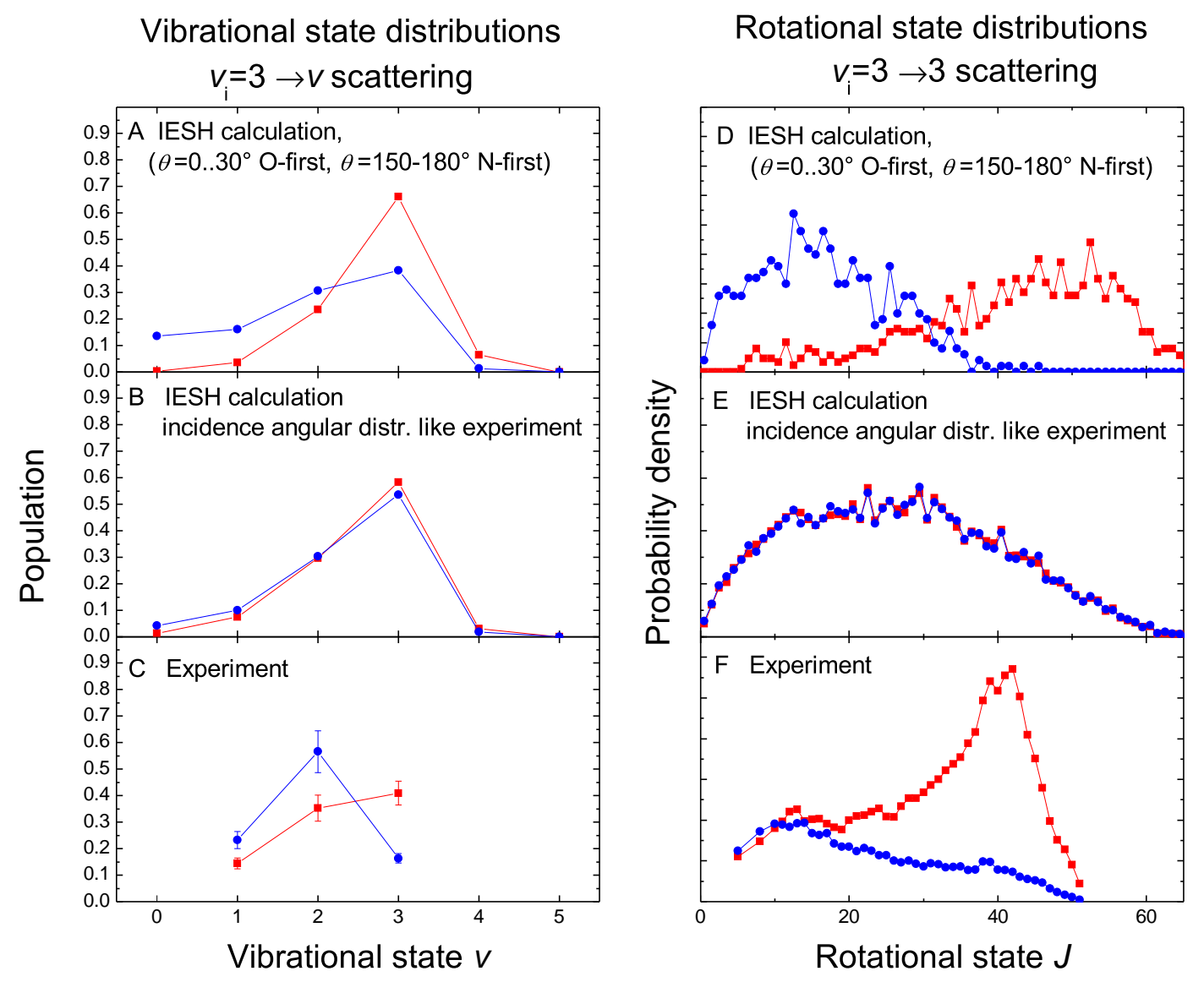

Figure 6.21: Comparison of IESH calculations to experimental data for the scattering of NO $v=3$ from Au(111) - (left) Comparison betweeen calculated and experimental vibrational state distributions for $\mathrm{NO}(v=3 \rightarrow v)$ scattering. (right) Comparison of theory and experiment for the rotational state distributions generated in the vibrationally elastic $v=3 \rightarrow 3$ scattering channel. Red colour indicates O-first orientation, while blue colour indicated $\mathrm{N}$-first orientation. The different panels are described in the text. Calculations have been performed by Christof Bartels. 
adiabatically or non-adiabatically. Furthermore, the rotational rainbow structure is (at least under realistic incident angular distributions) not captured. The adiabatic PES for the calculations was constructed by fitting energy values from DFT (PW91 functional).(67, 70) DFT has difficulties in describing electron transfer, because the electrons are treated as a fluid. Furthermore, recent results (162) suggest severe problems in the fit of the DFT data, because results from ab initio molecular dynamics differ significantly from calculations with the fitted PES.

Due to these deficiencies in the PES, it is too early to answer the question, whether part 2) of the calculation - the non-adiabatic propagation - is in general capable of describing the large vibrational energy transfer observed in the experiment, especially at high incidence translational energy.

\subsubsection{Electronic friction (EF) model from the Saalfrank group}

The IESH calculations discussed in last last subsection are not the only theoretical work predicting the wrong trend on how the vibrational energy transfer depends on the incidence translational energy. Based on a two-dimensional $V(z, r)^{1}$, quantum mechanical study with electronic friction (EF), Monturet and Saalfrank also suggested, that the vibrational relaxation should decrease with incidence translational energy (see section 2.4.3).(69) The reason for this prediction was that the lower translational energy increases the interaction time between the molecule and the surface. Within the EF model, the vibrational relaxation is calculated as a rate $\Gamma_{v \rightarrow v-1}$. In the harmonic version of the theory, the relaxation occurs stepwise $(\Delta v=-1)$ and the rate constants are related via

$$
\Gamma_{v \rightarrow v-1}=v \Gamma_{1 \rightarrow 0} .
$$

The experimental observation, that the vibrational relaxation becomes more effective with increasing translational energy, can thus only be explained in case the vibrational rate constants strongly depend on the incidence translational energy.

\subsubsection{Monte Carlo wave packet study from Li and Guo}

An older study published in 2006 is a Monte Carlo wave packet study from Li and Guo.(66) The Monte Carlo study allows a wave packet to jump between a neutral and

\footnotetext{
${ }^{1} z$ is the molecule-surface distance and $r$ the NO bond length.
} 
an anionic state to account for electron-transfer and uses two-dimensional, empirical PESs $V(z, r)$. The authors of this work state, that their model is rather empirical.(66) Interestingly, the model nevertheless made some very reasonable predictions regarding the incidence energy of translation dependence of the vibrational relaxation. In particular, the authors calculated vibrational state distributions for $v_{\mathrm{i}}=2$ and $v_{\mathrm{i}}=15$ at different incidence translational energies, which are qualitatively in fairly good agreement with our vibrational state distributions for $v_{\mathrm{i}}=3$ and $v_{\mathrm{i}}=16$, respectively (see Fig. 8 of Ref. (66) ). In particular, it was correctly predicted, that the vibrational energy transfer is promoted by incidence translation $E_{\text {trans }}^{\mathrm{i}}$ for low incidence vibrational states $\left(v_{\mathrm{i}}=2\right)$ and becomes independent of $E_{\mathrm{trans}}^{\mathrm{i}}$ for $v_{\mathrm{i}}=15$. The authors explained this behaviour arguing with the energetics of the underlying electron transfer very similar to our argumentation in section 6.3. They analyzed the crossing seam between neutral and anionic PESs and discuss the vibrational relaxation in terms of an energetic barrier towards electron transfer 1 An interesting feature of their model is that it yields the number of electron jumps in each collision trajectory. For $v_{\mathrm{i}}=15$ the wave-packet jumps between neutral and anionic states several times. As opposed to this, $v_{\mathrm{i}}=2$ collisions only have a $20 \%$ change for an electron jump to occur at all. This means, that the vibrational relaxation (for high vibrational states) is a multiple ET reaction within this model.

\subsection{Conclusions}

The new experimental work for scattering of vibrationally excited NO from $\mathrm{Au}(111)$ shows that incidence translational energy, $\mathrm{N}$-first molecular orientation and incidence vibrational energy drastically promote the vibrational energy transfer. These results can be understood by treating the vibrational relaxation as a process driven by transient electron transfer (ET). The complex interplay of the control parameters (vibrational, translational energy and orientation) results from the energetics of this ET process. Furthermore, collisions of NO with the surface produce strongly non-thermal rotational state distributions with pronounced rotational rainbow structure. The rotational state distributions are the result of a strongly anisotropic $\mathrm{NO} / \mathrm{Au}(111)$ interaction potential

\footnotetext{
${ }^{1}$ I actually overlooked the work from $\mathrm{Li}$ an Guo prior to the literature search for this PhD thesis. I apologize to the authors for not citing their work in my previous publications.
} 


\section{Vibrationally inelastic scattering of oriented NO from $\mathrm{Au}(111)$}

and can be modelled with classical molecular dynamics simulations. The effect of the rotational cooling upon vibrational relaxation is attributed to an anisotropy existing in the vibrational relaxation rates as well as in the rotational excitation.

The experimental data of this work further provides indications for a dynamical steering effect at low incidence translational energy and for a contribution of molecules that undergo bond dissociation.

So far, there exists no theoretical model capable of accurately predicting ro-vibrational state distributions under the various incidence conditions tested in this work. The current implementation of the only model with 6 degrees of freedom - which could thus in principle achieve this goal - seems to have major deficiencies in the adiabatic PES. These problems need to be solved before further conclusions can be made. The two dimensional study from Le and Guo accurately predicted the correct incidence energy of translation dependence of the vibrational relaxation. The study suggest that the vibrational relaxation of highly vibrationally excited $\mathrm{NO}$ scattering from $\mathrm{Au}(111)$ is the result of multiple electron transfer events. 


\section{7 \\ Production of a molecular beam of highly vibrationally excited CO using perturbations}

The interesting dynamics of highly vibrationally excited NO upon collisions with a $\mathrm{Au}(111)$ surface lead to the desire of extending these experiments to other diatomic molecules. Here, the $\mathrm{CO}$ molecule is an attractive candidate and its dynamics in gassurface interactions in low vibrational states have been extensively studied.(45, 163) We thus developed a new approach, that we call pump-pump-perturb and dump (P3D), which allows for the generation of an intense molecular beam of $\mathrm{CO} \mathrm{X}^{1} \Sigma^{+}$in high vibrational states (we tested the generation of $v=17$ and $v=18$ ). The approach uses the effect of intensity borrowing from molecular perturbations in order to transfer population to the vibrationally excited target state by multi-step optical pumping via formally dipole forbidden singlet-triplet electronic transitions. The success of the approach is demonstrated by laser induced fluorescence and REMPI spectroscopy. The idea for the project originates from Prof. Robert W. Field, who spend part of his sabbatical in 2011/2012 in Göttingen and helped in developing the pumping scheme and understanding the experimental data. We published some of the content from this chapter in J. Chem. Phys.(11). 


\subsection{Introduction and description of the concept}

Molecular beam scattering is a key experimental technique for studying quantum-state resolved gas phase as well as gas-surface reaction dynamics.(164, 165, 166, 167) Since the development of Stimulated Emission Pumping (SEP)(116, 117), it is also possible to study the dynamics of molecules carrying several eV of vibrational energy. SEP has been proven to be a very successful technique to study the spectroscopy of excited molecules including $\mathrm{I}_{2}, \mathrm{C}_{2} \mathrm{H}_{2}, \mathrm{CH}_{2} \mathrm{O}, \mathrm{NO}, \mathrm{HCN}, \mathrm{H}\left(\right.$ or D)FCO, HCP, Tropolone, $\mathrm{CS}_{2}$, $\mathrm{SO}_{2}, \mathrm{SCCl}_{2}, \mathrm{CH}_{2} \mathrm{O}, \mathrm{HCO}$, and $\mathrm{O}_{2}$ (see Ref.(11, 117, 168) and references therein). The use of SEP to excite molecules to higher vibrational states with high molecular beam densities in order to perform scattering experiments with surfaces or other gas phase molecules has so far only been demonstrated with a much smaller number of molecules: $\mathrm{O}_{2}, \mathrm{NO}$, and $\mathrm{CH}_{2} \mathrm{O}$.

With respect to the $\mathrm{CO}$ molecule, highly vibrationally excited molecules in the electronic ground state $\mathrm{X}^{1} \Sigma^{+}$have been generated by electron impact desorption from transition metal surfaces (169) and by energy pooling of CO molecules adsorbed on $\mathrm{NaCl}$ upon infrared irradiation (170, 171). Of course, both of these methods cannot be used for a state-specific preparation of highly vibrationally excited CO for scattering experiments.

Fig. 7.1 shows the optical pumping scheme we used to generate a molecular beam of highly vibrationally excited CO. The first electronically excited singlet state in $\mathrm{CO}$ is the $\mathrm{A}^{1} \Pi$ state. This electronic state can in principle be directly accessed via $\mathrm{A}^{1} \Pi \leftarrow \mathrm{X}^{1} \Sigma^{+}$, which is called the 4 th-positive system (172) and has a very strong transition strength (173). Unfortunately, this band lies deep in the vacuum UV and the generation of tunable, pulsed vacuum UV radiation is experimentally challenging (it is typically done by four-wave mixing in gas cells). The idea of P3D in the CO molecule is to use electronic triplet states in multi-step optical pumping. While optical transitions between singlet and triplet states are nominally spin-forbidden, some transitions can gain significant oscillator strength due to intensity borrowing from molecular perturbations. Specifically, spin-orbit mixing between $a^{3} \Pi_{1}$ and $A^{1} \Pi$ (remote perturber) allows for accessing the $\mathrm{a}^{3} \Pi_{1}$ state via Cameron band excitation (174) $\mathrm{a}^{3} \Pi_{1}\left(v^{\prime}=0\right) \leftarrow \mathrm{X}^{1} \Sigma^{+}\left(v^{\prime \prime}=0\right)$, which we call $\operatorname{pump}_{1}\left(\lambda_{1}\right)$ as shown in Fig. 7.1 


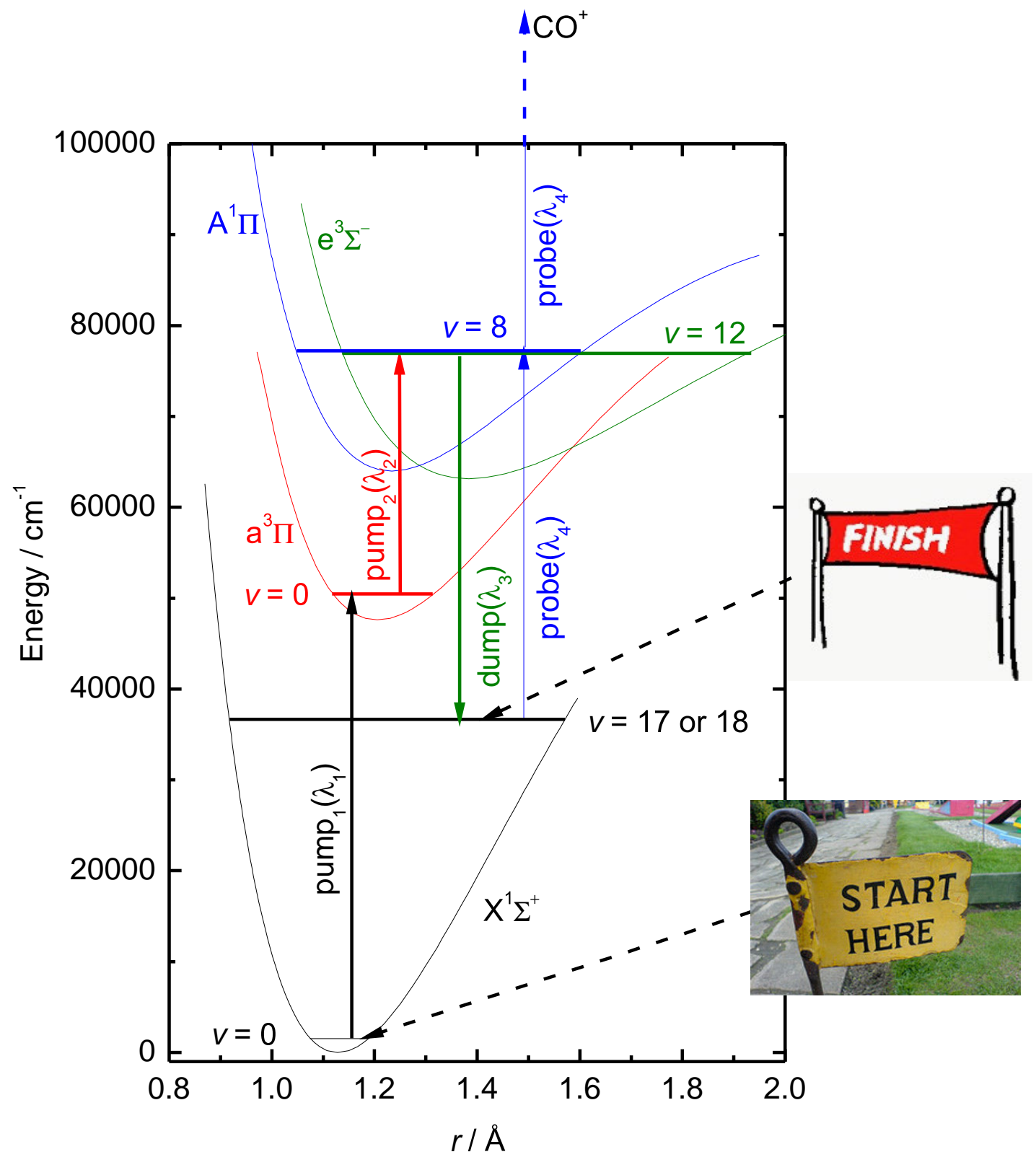

Figure 7.1: P3D concept for CO in a potential energy diagram. - $\mathrm{Pump}_{1}\left(\lambda_{1}\right)$ excites to $v=0$ of the metastable triplet state $\left(\mathrm{a}^{3} \Pi_{1}\right)$, shown as black arrow. The second pump step $\operatorname{pump}_{2}\left(\lambda_{2}\right)$ (red arrow) excites to a specific roational level of the predominantly $\mathrm{e}^{3} \Sigma^{-}(v=12)$ state, which is interacting with $\mathrm{A}^{1} \Pi(v=8)$ via spin-orbit interaction. From there, a third laser pulse $\left(\operatorname{dump}\left(\lambda_{3}\right)\right)$ can be used to enhance emission to a single ro-vibrational state $(v>>0)$ in $\mathrm{X}^{1} \Sigma^{+}$, green arrow. This population can be probed via $(1+1)-$ REMPI spectroscopy, e.g. via $\mathrm{A}^{1} \Pi(v=8)$ (blue arrow). 
We then excite to a specific rotational state in $\mathrm{e}^{3} \Sigma^{-}(v=12)$ via a spin allowed triplettriplet transition $\mathrm{e}^{3} \Sigma^{-}(v=12) \leftarrow \mathrm{a}^{3} \Pi_{1}(v=0)\left(\operatorname{pump}_{2}\left(\lambda_{2}\right)\right)$. At low $J$, the predominantly $\mathrm{e}^{3} \Sigma^{-}(v=12)$ state has significant $\mathrm{A}^{1} \Pi(v=8)$ character as these states are near degenerate and interact via spin-orbit interaction. The mixed $\mathrm{e}^{3} \Sigma^{-}(v=12) \sim$ $\mathrm{A}^{1} \Pi(v=8)$ state has a $\mu s$-lifetime and emits not only back to $\mathrm{a}^{3} \Pi$ (following the Franck-Condon factors of the $\mathrm{e}^{3} \Sigma^{-}(v=12) \rightarrow \mathrm{a}^{3} \Pi(v)$ system $)$, but also to vibrational states of $\mathrm{X}^{1} \Sigma^{+}$(following the Franck-Condon factors of the $\mathrm{A}^{1} \Pi(v=8) \rightarrow \mathrm{X}^{1} \Sigma^{+}(v)$ system). This effect is known as intensity borrowing (175) and is explained in Fig. 7.2 Similar to SEP, emission to a specific ro-vibrational state in $\mathrm{X}^{1} \Sigma^{+}$can be enhanced with another laser pulse (dump step) and the population can be probed with REMPI spectroscopy.

\subsection{Experimental}

A pulsed supersonic molecular beam of rotationally cold $\mathrm{CO}$ molecules is generated by expanding mixtures of $\mathrm{CO}$ seeded in a carrier gas as given in Table 7.1.

Table 7.1: Different $\mathrm{CO} /$ carrier gas mixtures with the speed and translational energy $E_{\text {trans }}$ of molecular beams used for testing P3D.

\begin{tabular}{ccc}
\hline \hline mixture & speed $/(\mathrm{km} / \mathrm{s})$ & $E_{\text {trans }}^{\mathrm{i}} / \mathrm{eV}$ \\
\hline $20 \% \mathrm{CO}$ in $\mathrm{Kr}$ & 0.34 & 0.017 \\
$20 \% \mathrm{NO}$ in $\mathrm{Ar}$ & 0.50 & 0.037 \\
$10 \% \mathrm{CO}$ in $\mathrm{H}_{2}$ & 1.45 & 0.303 \\
\hline \hline
\end{tabular}

In the differential chamber (9 $\mathrm{cm}$ downstream from the expansion, see also Fig. 3.1) the laser beams for the pump 1 , pump 2 , and dump steps cross the molecular beam, which are overlapped in space and time. Population in different vibrational states of $\mathrm{X}^{1} \Sigma^{+}$ are probed with REMPI spectroscopy in the surface chamber $(26 \mathrm{~cm}$ distance from the nozzle).

Figure 7.3 shows an energy diagram describing the optical transitions relevant for P3D in more detail. The pump $p_{1}$ excitation produces population in $\mathrm{CO}^{3} \Pi_{1}(v=0, J=1)$ via Cameron band excitation(174), similar to previous work(108, 176). This is done 


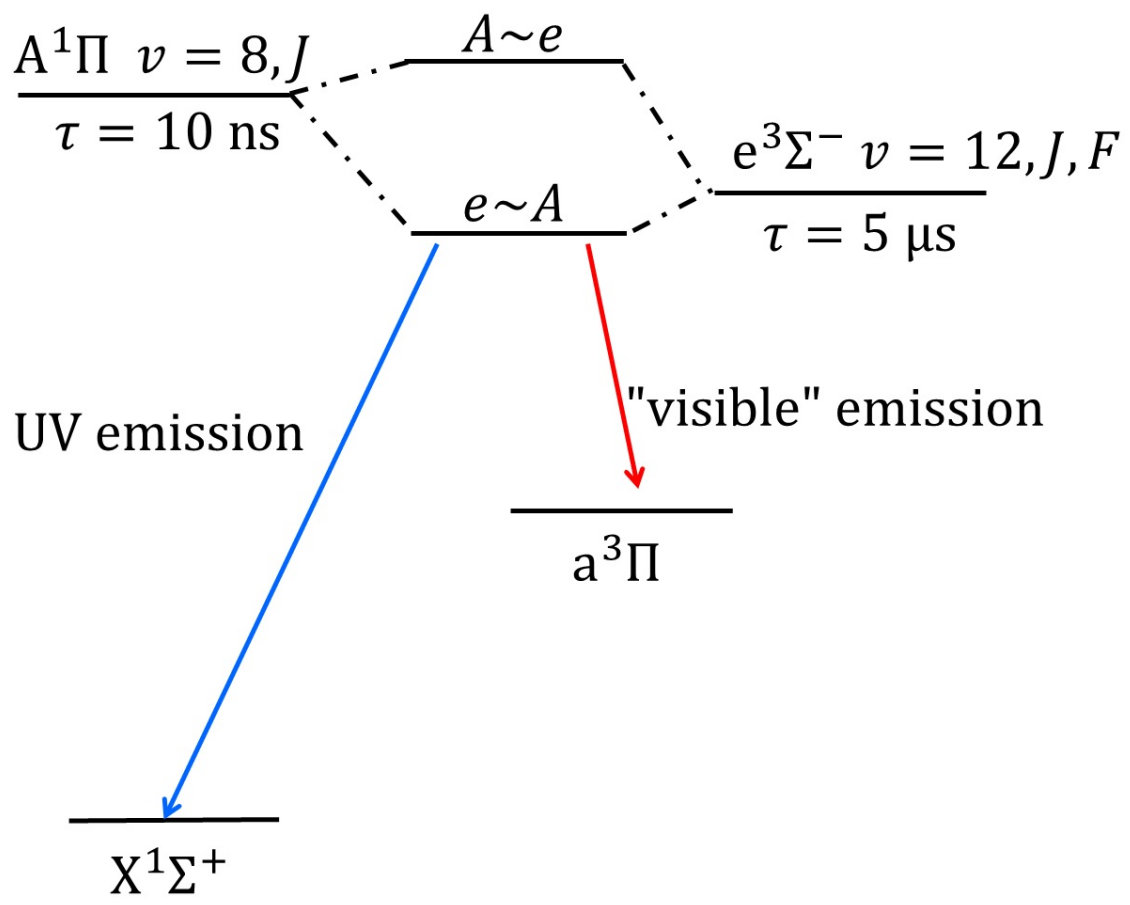

Figure 7.2: Explanation of the effect of intensity borrowing - Multistep optical pumping generates population in the mixed $\mathrm{e}^{3} \Sigma^{-}(v=12) \sim \mathrm{A}^{1} \Pi(v=8)$ state (abbreviated as $\mathrm{e} \sim \mathrm{A}$ in the figure). This state naturally emits in the visible back to $\mathrm{a}^{3} \Pi$ (visible emission via the Herman bands). However, the interaction with the $\mathrm{A}^{1} \Pi$ state also leads to a gain of oscillator strength to the electronic ground state $\mathrm{X}^{1} \Sigma^{+}$(UV emission via the 4th positive system). This effect is known as intenstity borrowing. Unperturbed rotational levels of $\mathrm{A}^{1} \Pi(v=8)$ state have a lifetime $\tau$ of $10 \mathrm{~ns}$. Unperturbed rotational levels of $\mathrm{e}^{3} \Sigma^{-}(v=12)$ have a lifetime of $5 \mu \mathrm{s}$. The lifetime of a mixed rotational state lies between these borderline cases and reflects the strength of the interaction between the zero-th order singlet and triplet states. 


\section{Production of a molecular beam of highly vibrationally excited CO using perturbations}

via either of the transitions

$$
\mathrm{a}^{3} \Pi_{1}(v=0, J=1,-, e) \stackrel{\lambda_{1}}{\longleftarrow} \mathrm{X}^{1} \Sigma^{+}(v=0, J=0,+, e) \text { at } \lambda_{1}=206.277 \mathrm{~nm},
$$

which is the $\mathrm{R}(0)$ line (black pump ${ }_{1}\left(\lambda_{1}\right)$ transition in Fig. 7.3) or via the $\mathrm{Q}(1)$ line (red $\operatorname{pump}_{1}\left(\lambda_{1}\right)$ transition in Fig. 7.3 .

$$
\mathrm{a}^{3} \Pi_{1}(v=0, J=1,+, f) \stackrel{\lambda_{1}}{\longleftarrow} \mathrm{X}^{1} \Sigma^{+}(v=0, J=1,-, e) \text { at } \lambda_{1}=206.293 \mathrm{~nm} .
$$

These transitions can be efficiently pumped with the home-built OPO laser system $(1 \mathrm{~mJ} /$ pulse at $3 \mathrm{~mm}$ beam diameter). The choice of the excitation transition in the $\operatorname{pump}_{1}\left(\lambda_{1}\right)$ step determines the parity of molecules prepared by P3D, as the parity selection rule $(+) \leftrightarrow(-)^{1}$ strictly holds for all optical pumping steps.

$\operatorname{Pump}_{2}\left(\lambda_{2}\right)$ excites CO to $\mathrm{e}^{3} \Sigma^{-}$via the Herman bands (178),

$$
\mathrm{e}^{3} \Sigma^{-}(v=12) \stackrel{\lambda_{2}}{\longleftarrow} \mathrm{a}^{3} \Pi_{1}(v=0, J=1) \text { at } \lambda_{2} \approx 368 \mathrm{~nm},
$$

to low rotational levels that interact (perturb step) via local spin-orbit interaction with $\mathrm{A}^{1} \Pi(v=8)$,

$$
\mathrm{e}^{3} \Sigma^{-}(v=12) \sim \mathrm{A}^{1} \Pi(v=8) .
$$

The interaction between these states has been documented in the literature (174, 179, 180) and is quantitatively treated in Appendix B.

As already mentioned, the mixed state naturally fluoresces via the Herman bands, and - due to intensity borrowing - also via the 4th-positive system. The Herman band emission back to vibrational levels in $\mathrm{a}^{3} \Pi$ lies in the visible and is detected with a photomultiplier tube (PMT, Hamamatsu R212 UH, 185-650 nm) in combination with a $400 \mathrm{~nm}$ longpass filter (Thorlabs FEL0400). Emission to $\mathrm{X}^{1} \Sigma^{+}$(4-th positive system) is detected with a UV sensitive PMT (Hamamatsu R7154, 160-320 nm). The emission

\footnotetext{
${ }^{1}$ According to Hougen (177), the rotational levels of all diatomic molecules can be classified as $(+)$ or $(-)$ according to their total parity, i.e., according to the behavior of the complete molecular wave function (apart from translation) when the laboratory-fixed Cartesian coordinates of all particles are replaced by their negatives. Often (also in this thesis) the word parity is sometimes used for the (e)/(f)-symmetry of a wavefunction (the total parity of the complete wavefunction exclusive of a $(-1)^{J}$ or $(-1)^{J-1 / 2}$ rotational factor for molecules with an even or odd number of electrons, respectively). For the CO molecule (even number of electrons) (+)-parity states have $(e)$-symmetry for even $J$ and $(f)$-symmetry for odd $J$. At the same time, $(-)$-parity states have $(f)$-symmetry for even $J$ and $(e)-$ symmetry for odd $J$. The parity selection rule $(+) \leftrightarrow(-),(+) \leftrightarrow(+)$ and $(-) \leftrightarrow(-)$ thus corresponds to the $(e) /(f)$-symmetry selection rule $(e) \leftrightarrow(e),(f) \leftrightarrow(f),(e) \leftrightarrow(f)$.
} 


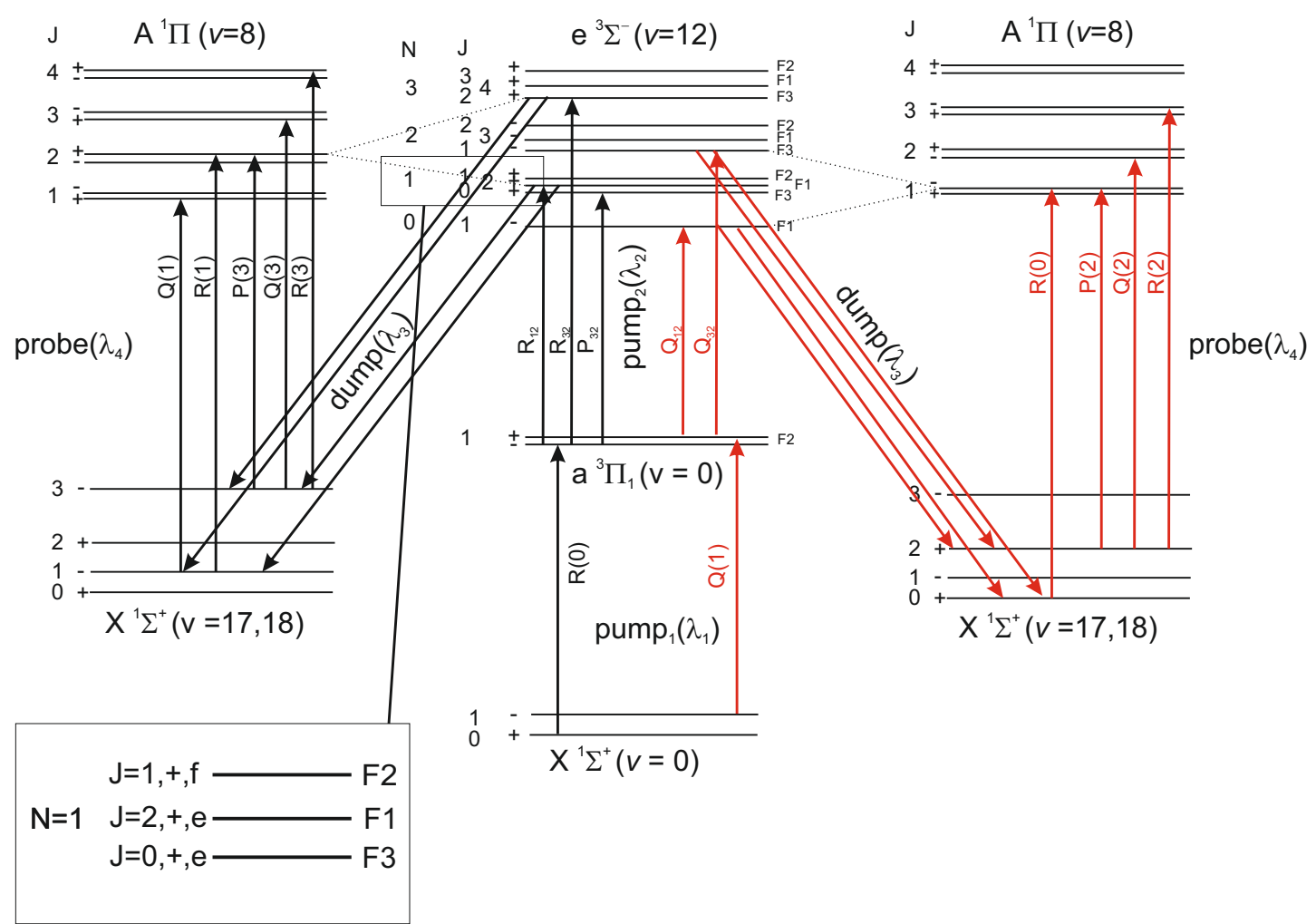

Figure 7.3: Energy level diagram and transitions important for P3D in CO - The $\operatorname{pump}_{1}\left(\lambda_{1}\right)$ step excites to the $J=1$ level of metastable $\mathrm{a}^{3} \Pi_{1}(v=0)$. By using the $\mathrm{Q}(1)$ or $\mathrm{R}(0)$ transition respectively, the $(+)$ or $(-)$ parity component (only separated by the $\Lambda$-splitting of $\left.\sim 0.013 \mathrm{~cm}^{-1}\right)$ is accessed. Pump $2\left(\lambda_{2}\right)$ excites to the perturbed $\mathrm{e}^{3} \Sigma^{-}(v=12)$ state. Note, that the $\mathrm{F}_{2}$ levels in $\mathrm{e}^{3} \Sigma^{-}(v=12)$ appear as dark states, as the spin selection rule $\Delta \Sigma=0$ does not allow them to be accessed from $\mathrm{a}^{3} \Pi_{1}\left(\mathrm{~F}_{2}\right.$ levels of $\mathrm{e}^{3} \Sigma^{-}$have $\Omega=|\Sigma|=1$ character, whereas the wave functions of $\mathrm{F}_{1}$ and $\mathrm{F}_{3}$ levels are linear combinations of $\Omega=\Sigma=0$ and $\Omega=|\Sigma|=1$ ). The dotted lines indicate the perturbations between levels of $\mathrm{e}^{3} \Sigma^{-}(v=12)$ and $\mathrm{A}^{1} \Pi(v=8)$ relevant to this work, interacting via spin-orbit interaction. The selection rules for the spin-orbit interaction are that the interacting states have same $J$ (as for any perturbation), same parity $((+)$ or $(-))$ and $\Delta \Lambda=-\Delta \Sigma= \pm 1$. Pumping of the perturbed rotational levels accessed in $\mathrm{e}^{3} \Sigma^{-}(v=12)$ results in fluorescence to high vibrational states in the electronic ground state $\mathrm{X}^{1} \Sigma^{+}(v \gg 0)$ (the exact distribution among vibrational states is given by FranckCondon factors times the cubed transition frequency (see Fig. 7.6) following the selection rules $\Delta J=0, \pm 1$ and the parity selection rule $(+) \leftrightarrow(-)$. These selection rules also apply for $(1+1)$ REMPI spectroscopy via the predominantly $\mathrm{A}^{1} \Pi$ state, which can be used to probed the population in $\mathrm{X}^{1} \Sigma^{+}$. The population in a specific ro-vibrational level in $\mathrm{X}^{1} \Sigma^{+}$ can be enhanced with a dump laser. The colour coding is consistent with Figs. 7.4 and 7.5. Figure and figure description adapted from (11). Copyright 2012 American Chemical Society. 


\section{Production of a molecular beam of highly vibrationally excited CO using perturbations}

to $\mathrm{X}^{1} \Sigma^{+}$follows the Franck-Condon factors (multiplied with the cubed transition frequency) of the $\mathrm{A}^{1} \Pi(v=8) \rightarrow \mathrm{X}^{1} \Sigma^{+}(v)$ system, as shown in Fig. 7.6. In this work, we probe population generated in the vibrational states $v=17$ and $v=18$ of $\mathrm{X}^{1} \Sigma^{+}$via $(1+1)$ REMPI spectroscopy,

$$
\mathrm{CO}^{+}+\mathrm{e}^{-} \stackrel{\lambda_{4}}{\longleftarrow} \mathrm{A}^{1} \Pi(v=8) \stackrel{\lambda_{4}}{\longleftarrow} \mathrm{X}^{1} \Sigma^{+}(v=17,18)
$$

at $\lambda_{4} \sim 234 \mathrm{~nm}$ for $v=17$ and $\lambda_{4} \sim 244 \mathrm{~nm}$ for $v=18$. Population transfer to specific ro-vibrational states in $\mathrm{X}^{1} \Sigma^{+}$can be enhanced in a $\operatorname{dump}\left(\lambda_{3}\right)$ step

$$
\mathrm{A}^{1} \Pi(v=8) \stackrel{\lambda_{3}}{\longrightarrow} \mathrm{X}^{1} \Sigma^{+}(v \gg 0)
$$

at $\lambda_{3} \sim 234 \mathrm{~nm}$ for $v=17$ and $\lambda_{3} \sim 244 \mathrm{~nm}$ for $v=18 . \operatorname{Pump}_{2}\left(\lambda_{2}\right), \operatorname{dump}\left(\lambda_{3}\right)$ and $\operatorname{probe}\left(\lambda_{4}\right)$ steps were performed with different frequency doubled, Nd:YAG pumped dye lasers (Sirah PRSC-DA-24, CSTR-LG-24, and CSTR-DA-24, respectively). The CSTR-LG-24 laser was borrowed from the beamer 1 setup. All of them have a $3 \mathrm{GHz}$ bandwidth. The $\operatorname{pump}_{2}\left(\lambda_{2}\right)$ step was performed with a power of $0.5 \mathrm{~mJ} /$ pulse $(5 \mathrm{~mm}$ beam diameter). The dump and probe steps were both performed with $1 \mathrm{~mJ} /$ pulse at $3 \mathrm{~mm}$ and $5 \mathrm{~mm}$ beam diameter, respectively. All laser pulses were linearly polarized parallel to the plane defined by the molecular beam and the laser beams ( $\mathrm{p}-$ polarization).

\subsection{Results}

The spin-orbit interaction between the $\mathrm{e}^{3} \Sigma^{-}(v=12)$ and the $\mathrm{A}^{1} \Pi(v=8)$ state, which have accidental near degeneracy at low $J$, can be demonstrated with laser induced fluorescence (LIF) spectroscopy (see Fig. 7.4). For these spectra, molecules were pumped to either the $(+)$ or the $(-)$-parity components of $\mathrm{a}^{3} \Pi_{1}(v=0, J=1)$, and the wavelength of the $\operatorname{pump}_{2}\left(\lambda_{2}\right)$ laser was scanned. Thereby, we either monitored the visible Herman band emission (fluorescence back to $\mathrm{a}^{3} \Pi$ ) or the ground state emission in the UV (4th positive system). Strong ground state emission (which is only possible due to intensity borrowing from molecular perturbations) is observed for all pumped transitions, except for $P_{32}(1, e)$. This is because the $\mathrm{e}^{3} \Sigma^{-}(v=12, J=0)$ level accessed by this transitions cannot interact with $\mathrm{A}^{1} \Pi(v=8)$ due to the perturbations selection rule $\Delta J=0$. This is because $\mathrm{A}^{1} \Pi$ (the $\mathrm{A}^{1} \Pi$ has $\Lambda=1$ and thus has rotational levels 
with $J \geq 1$ only) does not have a $J=0$ level and the selection rule $\Delta J=0$ can not be fulfilled. We also performed similar LIF experiments via $\mathrm{e}^{3} \Sigma^{-}(v=13)$, where accidental near degeneracies with $\mathrm{A}^{1} \Pi$ are not present at low $J$ (see the orange line in the perturbation diagram of $\mathrm{CO}$ in Fig. B.2 on page 168). In this case, no UV emission to $\mathrm{X}^{1} \Sigma^{+}$was detected, reflecting the absence of intensity borrowing from singlet-triplet mixing.

The degree of mixing can be further characterized by measuring the lifetimes of the

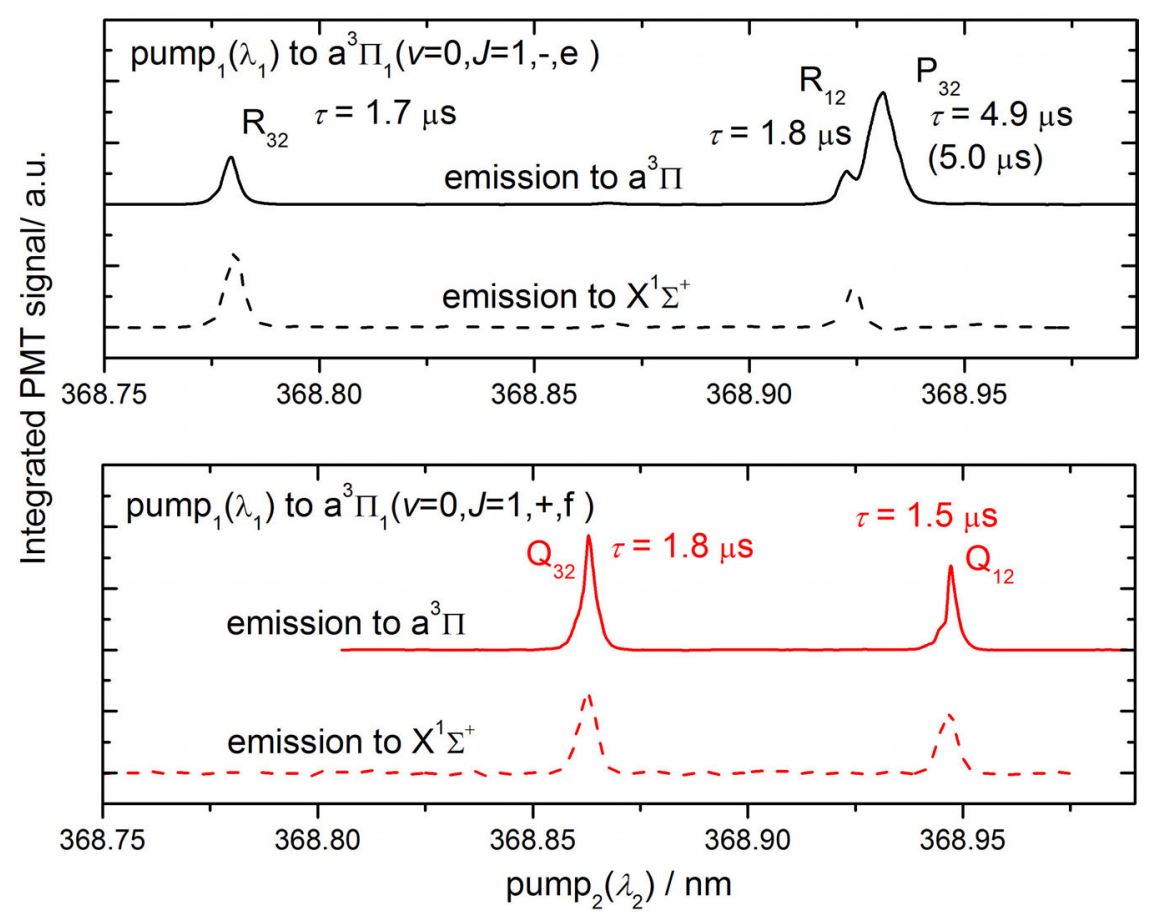

Figure 7.4: Laser induced fluorescence (LIF) spectra demonstrating the spinorbit interaction $\mathrm{e}^{3} \Sigma^{-}(v=12) \leftrightarrow \mathbf{A}^{1} \Pi(v=8)$ - The top panel shows LIF spectra of the $\mathrm{e}^{3} \Sigma^{-}(v=12) \leftarrow \mathrm{a}^{3} \Pi_{1}(v=0)$ band after excitation to the $(-)$-parity state of $\mathrm{a}^{3} \Pi_{1}(v=0)$ in $\operatorname{pump}_{1}\left(\lambda_{1}\right)$; the bottom panel after excitation to the corresponding $(+)$-parity state. Fluorescence monitoring visible $\mathrm{e}^{3} \Sigma^{-} \rightarrow \mathrm{a}^{3} \Pi$ emission is shown as solid lines, whereas the $\mathrm{e}^{3} \Sigma^{-} \rightarrow \mathrm{X}^{1} \Sigma^{+}$emission to the ground state in the UV is shown as dashed lines. Interestingly, the $P_{32}(1 e)$ line only appears in the visible spectrum, reflecting the absence of spin-orbit interaction for the $J=0$ level accessed by this transition. Note, that mixed states also have shorter radiative lifetimes $\tau$ (given as labels next to the transitions) compared to the deperturbed lifetime of $5 \mu$ s for the $\mathrm{e}^{3} \Sigma^{-}$state. Figure adapted from reference (11). The wavelength was shifted to accurate line positions measured by A. Meling (181). 


\section{Production of a molecular beam of highly vibrationally excited CO using perturbations}

mixed $\mathrm{A}^{1} \Pi(v=8)$ and $\mathrm{e}^{3} \Sigma^{-}(v=12)$ levels. These can in principle be obtained from the decay of the laser induced fluorescence. However, since the lifetime of these states is longer than the fly-out time of the detection volume $\left(1 \mathrm{~mm}^{3}\right)$ of the PMT optical imaging system, these measurements are challenging. We thus performed lifetime measurements $\tau_{\text {obs }}$ for gas mixtures with different velocities (as given in Table 7.1). We found that $\tau_{\text {obs }}^{-1}$ scaled linearly with the speed of the molecular beam and thus extrapolated $\tau_{\text {obs }}^{-1}$ to zero beam velocity, $\tau$. The lifetimes $\tau$ of the rotational levels accessed in the $\operatorname{pump}_{2}\left(\lambda_{2}\right)$ step are given as labels next to each transition in Fig. 7.4, as well as in Table 7.2,

Table 7.2: Lifetimes and mixing coefficients of the accessed $\mathrm{e}^{3} \Sigma^{-}(v=12) \sim \mathrm{A}^{1} \Pi(v=8)$ levels.

\begin{tabular}{cccc}
\hline \hline Rot. level & $\tau^{\mathrm{a}} / \mu \mathrm{s}$ (this work) & $\tau_{\text {calc }} \mathrm{b} / \mu \mathrm{s}$ & Mix. $\left.\mathrm{A}^{1} \Pi(v=8)^{\mathrm{c}}\right)$ \\
\hline$J=1, \mathrm{~F}_{3}$ & $1.8 \pm 0.3$ & 1.9 & 0.0033 \\
$J=1, \mathrm{~F}_{1}$ & $1.5 \pm 0.3$ & 1.8 & 0.0035 \\
$J=2, \mathrm{~F}_{3}$ & $1.7 \pm 0.3$ & 1.8 & 0.0037 \\
$J=2, \mathrm{~F}_{1}$ & $1.8 \pm 0.3$ & 2.1 & 0.0029 \\
$J=0, \mathrm{~F}_{3}$ & $4.9 \pm 0.9$ & 5.0 & 0 \\
\hline \hline
\end{tabular}

a) Experimental value from laser induced fluorescence decay extrapolated to zero beam velocity.

b) Derived from calculated mixing fraction of $\mathrm{A}^{1} \Pi(v=8)$ and the lifetimes of the deperturbed states of $10 \mathrm{~ns}$ for $\mathrm{A}^{1} \Pi$ and $5 \mu \mathrm{s}$ for $\mathrm{e}^{3} \Sigma^{-}$. c) Partial $\mathrm{A}^{1} \Pi(v=8)$ character calculated from spectroscopically determined molecular constants. The calculation is explained in Appendix B Results agree with literature (182).

As expected, the lifetimes of the mixed states are substantially shorter than the lifetime $(\tau=4.9 \pm 0.9 \mu \mathrm{s})$ of the unperturbed $\mathrm{e}^{3} \Sigma^{-}(v=12, J=0)$ level. Rotational levels of $\mathrm{e}^{3} \Sigma^{-}(v=13)$ were also found to have lifetimes in the order of $5 \mu \mathrm{s}$.

The lifetimes of the different rotational levels can also be calculated from spectroscopically determined molecular constants (183), taking the spin-orbit interaction between $\mathrm{e}^{3} \Sigma^{-}(v=12)$ and $\mathrm{A}^{1} \Pi(v=8)$ as well as the spin-uncoupling within the $\mathrm{e}^{3} \Sigma^{-}(v=12)$ state into account. These calculations can be found in Appendix B. The calculated lifetimes agree well with the experimental data (see Table 7.2). The table also gives calculated mixing fractions. The partial $\mathrm{A}^{1} \Pi(v=8)$ character of the predominantly $\mathrm{e}^{3} \Sigma^{-}(v=12, J=0)$ levels are relatively small (e.g. $0.33 \%$ fractional $\mathrm{A}^{1} \Pi(v=8)$ character in $\left.\mathrm{e}^{3} \Sigma^{-}\left(v=12, J=1, \mathrm{~F}_{3}\right)\right)$. 
Despite the small mixing coefficient, the mixed $\mathrm{e}^{3} \Sigma^{-}(v=12) \sim A^{1} \Pi(v=8)$ states have a significant quantum yield $\phi_{\mathrm{X}}$ for ground state emission. The quantum yield for ground state fluorescence $\phi_{\mathrm{X}}$ to $\mathrm{X}^{1} \Sigma^{+}$(versus emission to $\mathrm{a}^{3} \Pi$ ) is given by ${ }^{1}$

$$
\phi_{\mathrm{X}} \approx 1-\frac{\tau}{\tau_{\mathrm{e}}}=1-\frac{1.8 \mu \mathrm{s}}{4.9 \mu \mathrm{s}} \approx 0.6
$$

where $\tau_{\mathrm{e}}$ is the lifetime of the unperturbed $\mathrm{e}^{3} \Sigma^{-}$state and we used an averaged value of $\tau=1.8 \mu$ s for the lifetime of the mixed $\mathrm{F}_{1}$ and $\mathrm{F}_{3}$ states. Although the mixing fractions are quite small (e.g. $0.35 \%$ fractional $\mathrm{A}^{1} \Pi$ character in $\mathrm{e}^{3} \Sigma^{-}\left(v=12, J=1, \mathrm{~F}_{1}\right)$, see Table 7.2), a larger fraction of spontaneous emission goes to $\mathrm{X}^{1} \Sigma^{+}$(4th-positive band) compared to the spin-allowed Herman band emission to $a^{3} \Pi$. This is because the lifetime of the unperturbed $A^{1} \Pi$ state of $10 \mathrm{~ns}$ is by a factor of 200 shorter than the lifetime of the $\mathrm{e}^{3} \Sigma^{-}$state.(184)

Figure 7.5 shows $(1+1)$-REMPI spectra probing the optically prepared population in the $\mathrm{X}^{1} \Sigma^{+}(v=17)$ state (very similar results were obtained for the $\mathrm{X}^{1} \Sigma^{+}(v=$ 18) state). Depending on the transitions used for $\operatorname{pump}_{1}\left(\lambda_{1}\right)$ and $\operatorname{pump}_{2}\left(\lambda_{2}\right)$ steps, spontaneous emission from the mixed states excited in this work always populates two rotational states in $\mathrm{X}^{1} \Sigma^{+}(v)$. These states in $\mathrm{X}^{1} \Sigma^{+}(v>>0)$ always have the opposite parity $\left((+) /(-)\right.$ parity) as the starting level in $\mathrm{X}^{1} \Sigma^{+}(v=0)$ excited in the $\operatorname{pump}_{1}\left(\lambda_{1}\right)$ step (compare Figures 7.5 and 7.3$)$. Excitation of $\operatorname{pump}_{1}\left(\lambda_{1}\right)=\mathrm{R}(0)$ and $\operatorname{pump}_{2}\left(\lambda_{2}\right)=\mathrm{R}_{32}(1, e)$ populates the rotational levels $J^{\prime \prime}=(1,-)$ and $(3,-)$, giving rise to five REMPI lines $\mathrm{R}(1), \mathrm{Q}(1), \mathrm{R}(3), \mathrm{Q}(3)$, and $\mathrm{P}(3)$ (black solid line in panel (A) of Fig. 7.5). Instead, excitation of $\operatorname{pump}_{1}\left(\lambda_{1}\right)=\mathrm{Q}(1)$ and $\operatorname{pump}_{2}\left(\lambda_{2}\right)=\mathrm{Q}_{32}(1, f)$ populates the rotational levels $J^{\prime \prime}=(0,+)$ and $(2,+)$, giving rise to four REMPI lines $\mathrm{R}(0), \mathrm{R}(2), \mathrm{Q}(2)$, and $\mathrm{P}(2)$ black solid line in panel (D)). At the used laser power, the dump laser increased the population in each of the accessed rotational levels in

\footnotetext{
${ }^{1}$ Equation 7.9 is derived as follows: The fluorescence rate constant of the mixed state $k_{\text {mixed }}=1 / \tau$ depends linearly on the partial mixing fractions $f_{\mathrm{e}}$ and $f_{\mathrm{A}}$ of the $\mathrm{e}^{3} \Sigma^{-}$state and $\mathrm{A}^{1} \Pi$ states with the deperturbed rate constants of $k_{\mathrm{e}}=1 / \tau_{\mathrm{e}}=1 /(4.9 \mu \mathrm{s})$ and $k_{\mathrm{A}}=1 / \tau_{\mathrm{A}}=1 /(10 \mathrm{~ns})$, respectively.$$
k_{\text {mixed }}=1 / \tau=f_{\mathrm{e}} k_{\mathrm{e}}+f_{\mathrm{A}} k_{\mathrm{A}}=f_{\mathrm{e}} / \tau_{\mathrm{e}}+f_{\mathrm{A}} / \tau_{\mathrm{A}}
$$

Only the fraction $f_{\mathrm{A}} k_{\mathrm{A}}$ leads to ground state emission to $\mathrm{X}^{1} \Sigma^{+}$. Thus, the quantum yield $\phi_{\mathrm{X}}$ is given by

$$
\phi_{\mathrm{X}}=\frac{f_{\mathrm{A}} k_{\mathrm{A}}}{f_{\mathrm{e}} k_{\mathrm{e}}+f_{\mathrm{A}} k_{\mathrm{A}}}=\frac{f_{\mathrm{A}} k_{\mathrm{A}}}{\left(1-f_{\mathrm{A}}\right) k_{\mathrm{e}}+f_{\mathrm{A}} k_{\mathrm{A}}} \stackrel{\text { Eq. } 7.7 .7}{=} \frac{\tau_{\mathrm{e}}-\tau}{\tau_{\mathrm{e}}-\tau_{\mathrm{A}}} \stackrel{\tau_{\mathrm{A}} \ll \tau_{\mathrm{e}}}{\approx} 1-\frac{\tau}{\tau_{\mathrm{e}}} .
$$

The fraction $1-\phi_{\mathrm{X}}$ is emitted to the $\mathrm{a}^{3} \Pi$ state.
} 

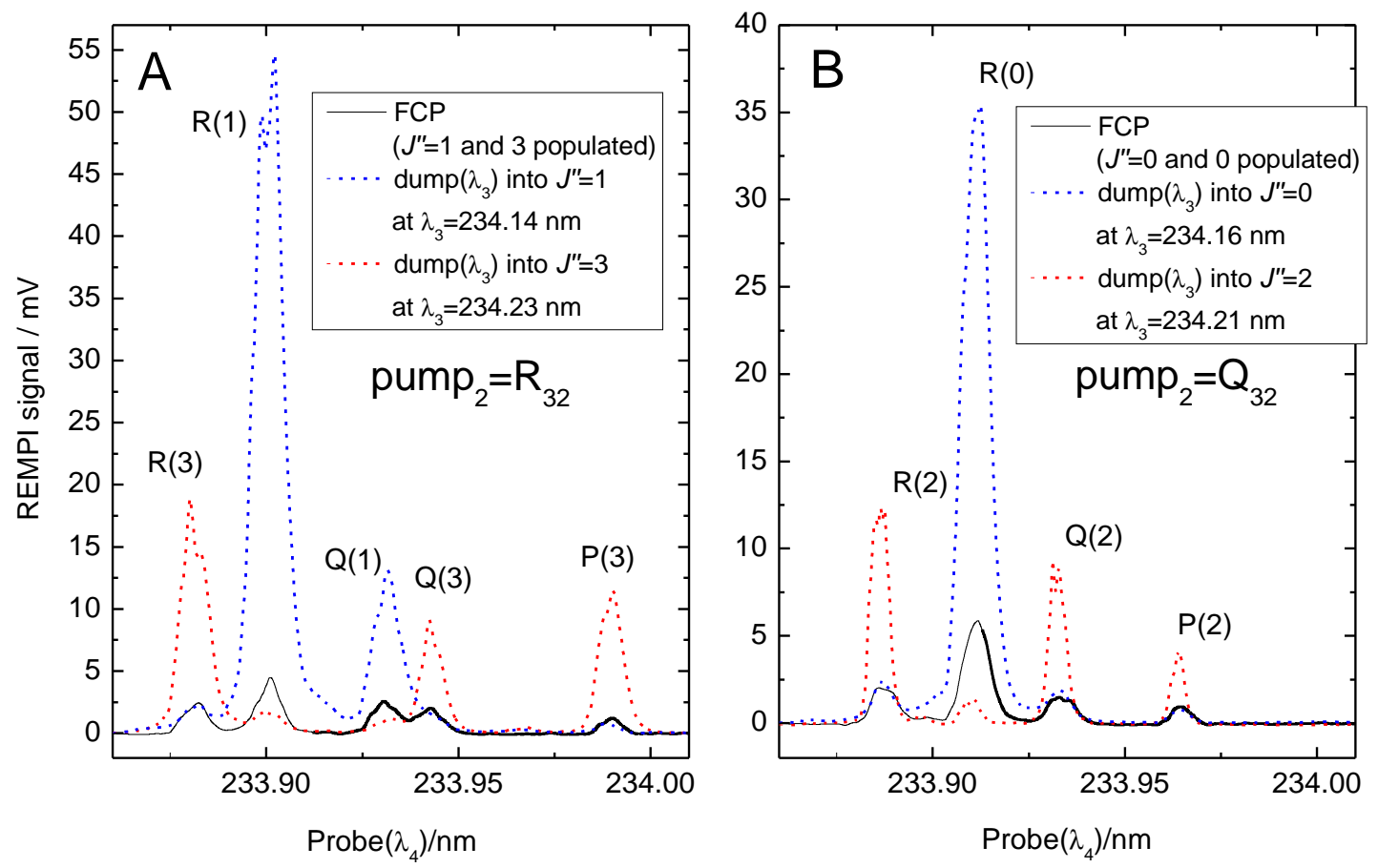

Figure 7.5: P3D demonstrated with REMPI spectroscopy - Comparison of REMPI spectra of $\mathrm{CO}(v=17)$ for different excitations in $\operatorname{pump}_{1}\left(\lambda_{1}\right)$ and $\operatorname{pump}_{2}\left(\lambda_{2}\right)$, with and without dump laser, probed by $(1+1)$ REMPI spectroscopy through $\mathrm{A}^{1} \Pi(v=8)$. Excitation of $\mathrm{R}(0)$ by $\operatorname{pump}_{1}\left(\lambda_{1}\right)$ and $\mathrm{R}_{32}(1, e)$ (compare Fig. 7.3 ) by pump ${ }_{2}\left(\lambda_{2}\right)$ (black solid line in panel (A)) gives rise to populations of $J^{\prime \prime}=(1,-)$ and $(3,-)$ in $\mathrm{CO} \mathrm{X}^{1} \Sigma^{+}(v=17)$. The REMPI spectrum was unchanged when using the $\mathrm{R}_{12}(1, e)$ line for $\operatorname{pump}_{2}\left(\lambda_{2}\right)$ instead. Excitation of Q(1) in $\operatorname{pump}_{1}\left(\lambda_{1}\right)$ and $\mathrm{Q}_{32}(1, f)$ (or $\mathrm{Q}_{12}(1, f)$ ) in pump ${ }_{2}\left(\lambda_{2}\right)$ (black solid line in panel (B)) results in population of $J^{\prime \prime}=(0,+)$ and $(2,+)$ instead. Using a dump $\operatorname{dum}_{3}\left(\lambda_{3}\right)$ pulse (dashed lines in each panel) enhances the population of a single ro-vibrational state. On average, the dump laser enhances the emission to a single ro-vibrational state by an average factor of $\sim 7$ compared to spontaneous emission from the predominantly $\mathrm{e}^{3} \Sigma^{-}$ level of the $\mathrm{e}^{3} \Sigma^{-}(v=12) / A^{1} \Pi(v=8)$ pair of interacting levels. The strongest amplification was observed for the $\mathrm{R}(1)$ transition (factor of $\sim 15$ ). The absolute intensities of REMPI spectra from panel (A) cannot be directly compared to those from panel (B), because measurements were performed on different days. 
$\mathrm{X}^{1} \Sigma^{+}(v=17)$ by an average factor of $\sim 7$ (dashed lines in Fig. 7.5). Wavelengths for the $\operatorname{dump}\left(\lambda_{3}\right)$ step are given as labels in each of the panels. Despite the strong amplification of the REMPI signal, we did not observe a depletion of the pump 2 LIF signal induced by the dump laser.

\subsection{Discussion}

The amplification of the REMPI signal by the dump laser clearly shows that we efficiently and state-selectively produced CO in $v=17$ and 18 .

Based on calculated Franck-Condon factors for the $\mathrm{A}^{1} \Pi(v=8) \rightarrow \mathrm{X}^{1} \Sigma^{+}(v)$ system (see Fig. 7.6), P3D can be extended to the production of $\mathrm{CO}$ in vibrational states up to $v=20$, corresponding to a vibrational energy of $4.7 \mathrm{eV}$. The generation of even higher vibrational states or states with unfavourable FCFs would require using other perturbations for P3D. In principle, any strong perturbation between $A^{1} \Pi$ and the states of the triplet manifold $\left(\mathrm{a}^{\prime 3} \Sigma^{+}, \mathrm{e}^{3} \Sigma^{-}, \mathrm{d}^{3} \Delta\right)$ occuring at low $J$-states can be exploited (see Figs. B.1 and B.2 of the Appendix). For example, Blokland et al. (185) recently used the mixed $\mathrm{A}^{1} \Pi\left(v^{\prime}=1, J=1,-\right) \sim \mathrm{d}^{3} \Delta_{1}\left(v^{\prime}=5\right)$ level to generate translationally cold (Stark decelerated) $\mathrm{CO} \mathrm{X}^{1} \Sigma^{+}(v=0, J=0)$ molecules, which become populated by spontaneous emission from the mixed state, which was - similar to this work accessed by two-step optical pumping via $\mathrm{a}^{3} \Pi_{1}$.

As already mentioned, pumping a rotational level of the $\mathrm{e}^{3} \Sigma^{-}(v=12) / \mathrm{A}^{1} \Pi(v=8)$ pair of interacting states, yields a quantum yield of ground state fluorescence of $\phi_{\mathrm{X}} \sim 60 \%$. From the calculated Franck-Condon factors of the $\mathrm{A}^{1} \Pi(v=8) \rightarrow \mathrm{X}^{1} \Sigma^{+}(v)$ system multiplied with the cubed transition frequencies (right panel of Fig. 7.6), it can be estimated, that a fraction of $6 \%$ of molecules emitted to $\mathrm{X}^{1} \Sigma^{+}$by spontaneous emission ends up in the vibrational target state $\mathrm{X}^{1} \Sigma^{+}(v=17)$. This population is approximately equally separated among two rotational states. Thus, pumping a mixed level in $\mathrm{e}^{3} \Sigma^{-}(v=12)$ leads to a quantum yield of approximately $0.6 \cdot 0.06 \cdot 0.5=1.8 \%$ in a specific rotational state of $\mathrm{X}^{1} \Sigma^{+}(v=17)$. The observation, that the dump lasers enhances the REMPI signal originating from such a rotational state by an average factor of 7 (see Fig. 7.5) suggests, that P3D transfers a fraction of $7 \times 1.8 \%=12 \%$ of 

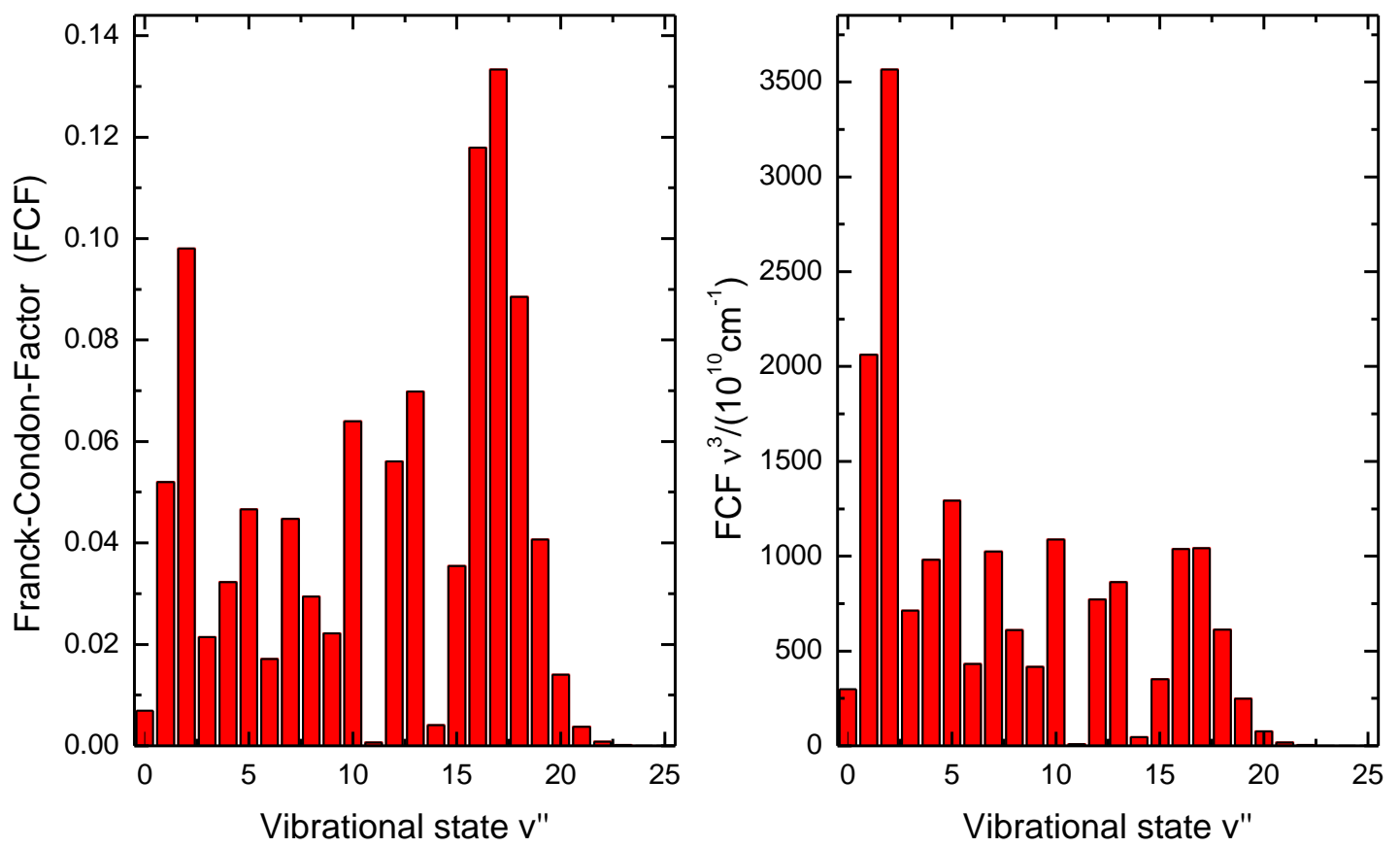

Figure 7.6: Franck-Condon-factors (FCF) of the $\mathrm{A}^{1} \Pi(v=8) \rightarrow \mathrm{X}^{1} \Sigma^{+}(v)$ system - (left) Franck-Condon-factors (FCFs) calculated with the programs RKR1 2.0 (186) and LEVEL 8.0 (187). They agree with those previously determined by Field in 1972 (183). (right) The FCFs are multiplied with the cubed transition frequency. This gives an estimate of the vibrational state distribution among vibrational states in $\mathrm{X}^{1} \Sigma^{+}$produced by spontaneous emission from mixed $\mathrm{e}^{3} \Sigma^{-}(v=12) \sim \mathrm{A}^{1} \Pi(v=8)$ levels. 
the population generated in $\mathrm{a}^{3} \Pi$ to the ro-vibrational target state 11 Thus, the dump step in P3D was not saturated, which is consistent with the observation of a lack of a (dump laser induced) fluorescence depletion in the LIF signal from the pump 2 step $2^{2}$ Here, additional work would be beneficial to fully characterize and optimize the transfer efficiency of P3D. A possible issue when increasing the dump laser power might be a laser induced loss of population due to ionization 3

In this work, we performed P3D via the predominantly triplet component of the $\mathrm{e}^{3} \Sigma^{-}(v=12) / \mathrm{A}^{1} \Pi(v=8)$ pair of interacting levels. An alternative approach was to perform the optical pumping via the predominantly singlet component of the same pair of mixed states. In this case, the $\operatorname{pump}_{2}\left(\lambda_{2}\right)$ step would be about 100 times weaker, but the dump $\left(\lambda_{3}\right)$ step could be easily saturated. It is possible, that this scheme leads to a better overall efficiency of population transfer.

\subsection{Conclusions}

For the first time, a molecular beam of $\mathrm{CO}$ molecules has been selectively prepared in a high vibrational state $(v=17,18)$. The state preparation is based on multi-step optical pumping exploiting well characterized singlet-triplet interactions. This is a key step for future studies on the vibrational dynamics of the molecule, e.g. in gas-surface collisions.

P3D is currently being implemented in another experimental setup (the Stark decelerator) in order to study collisions of highly vibrationally excited $\mathrm{CO}$ with a low work function (Cs-covered) $\mathrm{Au}(111)$ surface (181).

\footnotetext{
${ }^{1}$ This step of the calculation is simplified, because a more careful treatment would require to take into account, that the pump and dump steps are performed simultaneously and not sequentially.

${ }^{2}$ For example, SEP in the NO molecule was performed with fluorescence depletions in the order of $20 \%$ (see Fig. 5.3 on page 72 . However, the fluorescence depletion is in general only observed in a well optimized setup, where the beam profile of the dump laser is much larger, than that of the probe laser. In addition, fluorescence can also be generated by stray light (in particular for strong dipole-allowed transitions such as the pump $_{2}$ step in P3D). Thus, the lack of an observed fluorescence depletion in P3D can easily be an experimental artefact.

${ }^{3} \mathrm{~A}$. Mehling observed a REMPI signal induced by ionizing $\mathrm{a}^{3} \Pi_{1}$ molecules with the dump laser during the implementation of P3D on the Stark decelerator (181). The ionization probability is however believed to be rather small.
} 
7. Production of a molecular beam of highly vibrationally excited CO using perturbations 


\section{8}

\section{Outlook}

In this thesis, I present a lot of additional data for molecular beam surface scattering of $\mathrm{NO}$ with $\mathrm{Au}(111)$ and new ideas for their molecular level interpretation. Follow-up experiments that are currently being performed on that system by Bastian Krüger focus on the measurement of time-of-flight distributions of surface scattered molecules. I contributed to this research by designing a new ion detector, which is shown in Fig. 8.1. The main advantage of the new ion detector compared to the one used previously, is the increased ion detection volume in front of the surface. This allows for the measurement of very accurate angular and time-of-flight distributions of surface scattered molecules. Furthermore, the signal to noise ratio is improved by a factor $>10$ compared to the previous detector. With this new detector, the scattering experiments with NO prepared in excited vibrational states are planned to being extended to other surfaces (possibly $\mathrm{Ag}(111), \mathrm{Ge}(111)$, insulators or even nano-structures). This will answer the question of what aspects of the surface are relevant for the observed energy transfer between the molecular vibration and electronic degrees of freedom in the surface. My current understanding of this energy transfer is that it is governed by electron transfer and that the most important aspect of the surface for the vibrational energy transfer is the surface work function. Future experiments might prove this hypothesis.

More difficult than changing the surface, is changing the impinging gas-phase molecule in the experiment. As discussed in the last chapter of this thesis, we developed a new method for the preparation of $\mathrm{CO}$ in high vibrational states. Measuring vibrational state distributions (similar to the NO scattering experiments) with this technique will require large effort. Three important points need to be considered: First, REMPI 


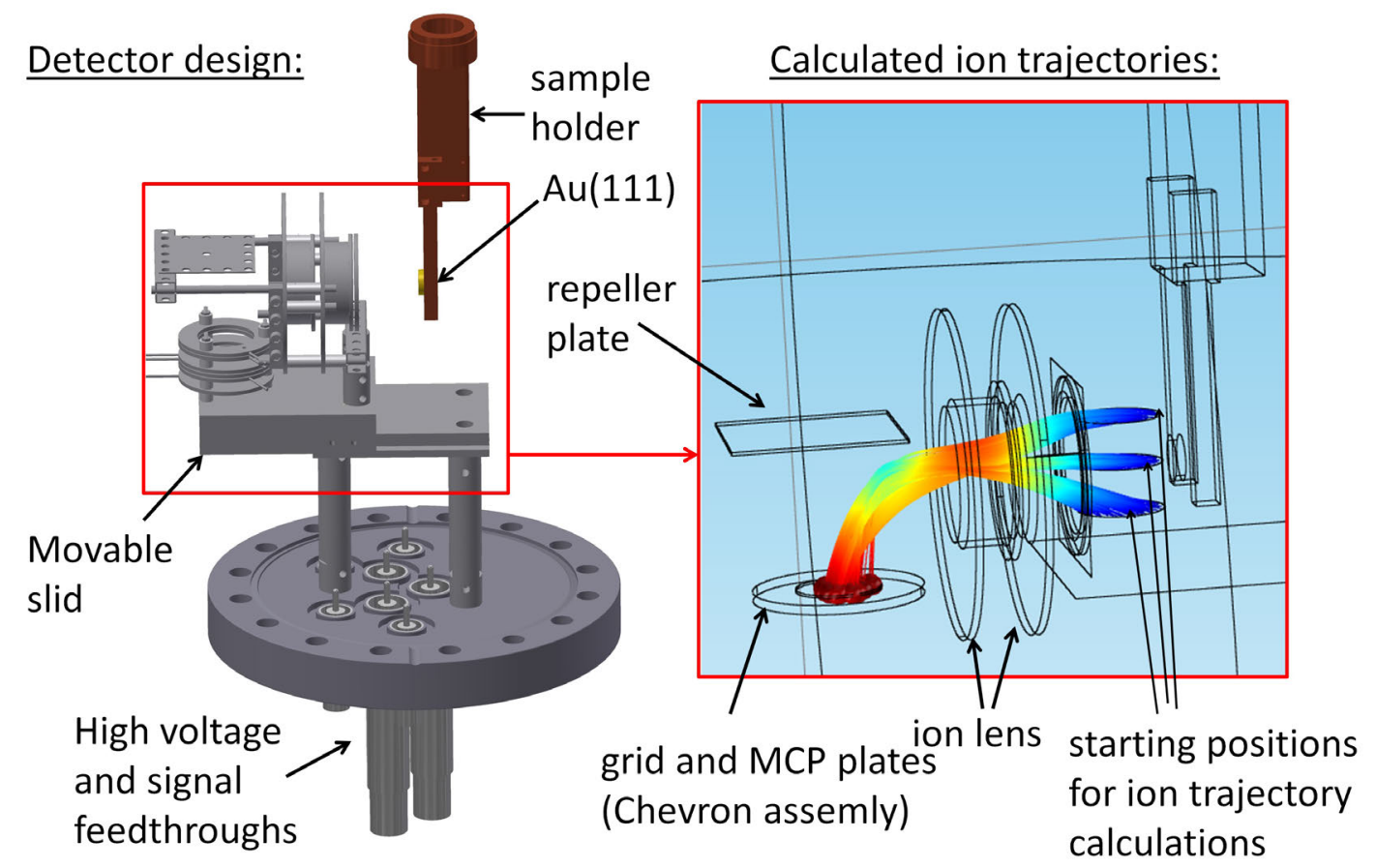

Figure 8.1: Design of the new ion detector - (left) Exported image from Autodesk Inventor showing the design of the ion detector. The detector sits on a movable slid for easy mounting in the optimal position. (right) Ion trajectories calculated with Comsol Multiphysics. At appropriate voltages, ions are efficiently guided to the detector, even when a large starting volume for the trajectories is chosen. This allows for an accurate determination of angular and time-of-flight distributions of surface scattered molecules. The large ion lens also shields the MCP from scattered light used for ionizing surface scattered molecules. 
schemes for the different vibrational states need to be tested. Furthermore, it might become necessary to improve quantum state purity (similar to the pump-dump-sweep approach in NO) 1 and the density (depending on the detection efficiency of the overall setup) of the incidence molecular beam. An easier first experiment is to probe the electron emission of highly vibrationally exited $\mathrm{CO}$ scattering from a low-work function $\mathrm{Au}(111)$ surface. This requires deflecting residual $\mathrm{a}^{3} \Pi$ molecules populated by optical pumping, as these are known to also generate electron emission.(57, 59, 60)

From the theory side, it would certainly be very beneficial to revisit the $\mathrm{NO} / \mathrm{Au}(111)$ system with improved ab initio models, taking the new experimental results into account. This work has already started.(162) Here, it can be used that some features of the calculations strongly depend on the electronic structure part of computational chemistry (e.g. the rotational structure and the translational inelasticity are mostly affected by the adiabatic PES) and not so much on the nuclear propagation part (which also contains the non-adiabatic effects).

\footnotetext{
${ }^{1}$ An alternative is a two-step optical preparation with STIRAP with vacuum UV directly via the $\mathrm{A}^{1} \Pi$ state, which has never been tested.
} 
8. Outlook 


\section{References}

[1] Y. Huang, C. T. Rettner, D. J. Auerbach, and A. M. WODTKE. Vibrational promotion of electron transfer Science, 290(5489):111-114, 2000. 10 2 23 [26 68

[2] N. Shenvi, S. Roy, And J. C. Tully. Dynamical steering and electronic excitation in NO scattering from a gold surface Science, 326(5954):829-832, 2009. 1

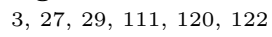

[3] T, SchäFER, N. BARTELS, N. Hocke, X. YANG, AND A, M. WODTKE. Orienting polar molecules without hexapoles: Optical state selection with adiabatic orientation Chem. Phys. Lett., 535:1 - 11, 2012. 3 $4 9 \longdiv { 5 7 } 5 8,60,61,62$

[4] N. Bartels, K. Golibrzuch, C. Bartels, L. Chen, D. J. Auerbach, A. M. WodtKe, and T. SChÄFer. Observation of orientation-dependent electron transfer in molecule-surface collisions Proc. Natl. Acad. Sci., 110(44):17738-17743, 2013. 3578187

[5] N. Bartels, K. Golibrzuch, C. Bartels, L. Chen, D. J Auerbach, A. M. Wodtke, and T. Schäfer. Dynamical steering in an electron transfer surface reaction: Oriented $\mathrm{NO}\left(v=3,0.08 \mathrm{eV}<E_{i}<0.89 \mathrm{eV}\right) \mathrm{re}-$ laxation in collisions with a $\mathbf{A u ( 1 1 1 )}$ surface $J$. Chem. Phys., 140(5):054710, 2014. 3 [5 $57 \quad 81 \quad 83$ 91 9394

[6] N. Bartels, B. C. Krüger, D. J. Auerbach, A. M. WodtKe, AND T. SCHÄFER. Controlling an electron-transfer reaction at a metal surface by manipulating reactant motion and orientation Angew. Chem. Int Ed., 53(50):13690-13694, 2014. 3 81 101 103 111 112113191

[7] B. C. Krüger, N. Bartels, C. Bartels, A. Kandratsenka, J. C. Tully, A. M. WodtKe, And T. Schäfer. NO vibrational energy transfer on a metal surface: Still a challenge to first-principles theory J. Phys. Chem. C, 119(6):3268-3272, 2015. 3 81 120 121 122

[8] T. Schäfer, N. Bartels, K. Golibrzuch, C. Bartels, H. KÖCKert, D. J. Auerbach, T. N. Kitsopoulos, AND A. M. WODTKE. Observation of direct vibrational excitation in gas-surface collisions of $\mathrm{CO}$ with $\mathrm{Au}(111)$ : a new model system for surface dynamics Phys. Chem. Chem. Phys., 15:1863-1867, 2013. 4.

[9] P. R. Shirhatti, J. Werdecker, K. Golibrzuch, A. M. WodTKe, and C. BARTels. Electron hole pair mediated vibrational excitation in $\mathrm{CO}$ scattering from
$\mathrm{Au}(111)$ : Incidence energy and surface temperature dependence $J$. Chem. Phys., 141(12):-, 2014. 4.

[10] K. Golibrzuch. Quantum-state resolved scattering of molecules from surfaces $\mathrm{PhD}$ thesis, Universtity of Goet-

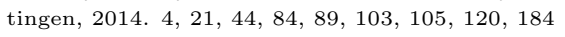

[11] N. Bartels, T. Schäfer, J. Hühnert, R. W. Field, And A. M. Wodtke. Production of a beam of highly vibrationally excited CO using perturbations $J$. Chem. Phys., 136(21):214201, 2012. 4 $127[128] 133$ 135

[12] A. W. Jasper, C. Zhu, S. Nangia, and D. G. Truhlar. Introductory lecture: Nonadiabatic effects in chemical dynamics Faraday Discuss., 127:1-22, 2004. 5] 7

113] M. H. Chowdhury, K. Aslan, S. N. Malyn, J. R. Lakowicz, AND C. D. GedDES. Metal-enhanced chemiluminescence J. Fluoresc., 16(3):295-299, 2006. 6

[14] S. InOUYe. Firefly luciferase: an adenylate-forming enzyme for multicatalytic functions Cell. Mol. Life Sci., 67(3):387-404, 2010. 6

[15] John C. Tully. Theoretical Chemistry Accounts chapter Perspective on "Zur Quantentheorie der Molekeln", pages 173-176. Springer Berlin Heidelberg, 2001. 6 7

[16] M. Born and R. Oppenheimer. Zur Quantentheorie der Molekeln Ann. Phys., 389(20):457-484, 1927. 7

[17] E. E. Nikitin. Nonadiabatic transitions: What we learned from old masters and how much we owe them Annu. Rev. Phys. Chem., 50(1):1-21, 1999. 8

[18] A. W. Kleyn, J. Los, and E. A. Gislason. Vibronic coupling at intersections of covalent and ionic states Phys. Rep., 90(1):1-71, 1982. $8[9$

[19] C. Wittig. The Landau-Zener formula J. Phys. Chem. B, 109(17):8428-8430, 2005. 9]

[20] C. ZENER. Non-adiabatic crossing of energy levels Proc. Roy. Soc. A, 137(833):696-702, 1932. 9

21] L. D. LANDAU. A theory of energy transfer II Phys. Z. Sowjetunion, 2(46), 1932. 9

22] E. C. G. Stueckelberg. Theorie der unelastischen Stösse zwischen Atomen Helv. Phys. Acta, 5(6):369-422, 1932. 9

[23] J. B. Hasted And. Physics of atomic collisions. Butterworth \& Co. Ltd, 1964. 9

24] T. S. Rose, M. J. Rosker, and A. H. Zewail. Femtosecond realtime observation of wave packet oscillations (resonance) in dissociation reactions $J$. Chem. Phys., 88(10):6672-6673, 1988. 9010

[25] H. Schmidt, J. von Vangerow, F. Stienkemeier, A. S. Bogomolov, A. V. Baklanov, D. M. Reich, W. Skomorowski, C. P. Koch, And M. Mudrich. Predissociation dynamics of lithium iodide J. Chem. Phys., 142(4):044303, 2015. 10 


\section{REFERENCES}

[26] Y.-C. Han, K.-J. Yuan, W.-H. Hu, and S.-L. Cong. Control of photodissociation and photoionization of the NaI molecule by dynamic Stark effect $J$. Chem. Phys., 130(4):044308, 2009. 10

[27] E. I. Dashevskaya, E. E. Nikitin, and J. Troe. The vibrational relaxation of $\mathrm{NO}$ in Ar: tunneling in a curve-crossing mechanism Phys. Chem. Chem. Phys., 17:151-158, 2015. 10

[28] A. Gross. Theoretical surface science (2nd edition). Springer-Verlag Berlin Heidelberg, 2009. 11,13

[29] Bengt I. Lundovist, Anders Hellman, and Igor Zorić. Handbook of surface science $\mathbf{3}$, chapter 10: Electron transfer and nonadiabaticity, pages 429 - 524. Elsevier, 2008. 11,13

[30] E. Hasselbrink. How non-adiabatic are surface dynamical processes? Curr. Opin. Solid State Mater. Sci., 10(3-4):192-204, 2006. 11

[31] A. M. Wodtke, J. C. Tully, and D. J. Auerbach. Electronically non-adiabatic interactions of molecules at metal surfaces: Can we trust the BornOppenheimer approximation for surface chemistry? Int. Rev. Phys. Chem., 23(4):513-539, 2004. 11

[32] A. M. Wodtke, D. Matsiev, and D. J. Auerbach. Energy transfer and chemical dynamics at solid surfaces: The special role of charge transfer Prog. Surf. Sci., 83(3):167-214, 2008. 11$] 13$

[33] C. Bartels, R. Cooper, D. J. Auerbach, and A. M. WODTKE. Energy transfer at metal surfaces: the need to go beyond the electronic friction picture Chem. Sci., 2(9):1647-1655, 2011. 11]

[34] B. Gergen, H. Nienhaus, W. H. Weinberg, and E. W. McFARLAND. Chemically induced electronic excitations at metal surfaces Science, 294(5551):25212523,2001 . 12

[35] O. Bünermann. H-atom scattering from Au (manuscript in preparation). High profile journal, 2015. 1213

[36] H. L. Skriver and N. M. Rosengaard. Surface energy and work function of elemental metals Phys. Rev. $B, 46: 7157-7168,1992$. 14

[37] P. J. Linstrom and W. G. Mallard, editors. NIST Chemistry WebBook, NIST Standard Reference Database Number 69 National Institute of Standards and Technology, Gaithersburg MD, retrieved November 11, 2014. 14

[38] F. K. Botz and R. E. Glick. Methane temporary negative ion resonances Chem. Phys. Lett., 33(2):279283,1975 . 14

[39] J. A. Appelbaum and D. R. Hamann. Variational calculation of the image potential near a metal surface Phys. Rev. B, 6(4):1122-1130, 1972. 15

[40] K. Golibrzuch, N. Bartels, D. J. Auerbach, and A. M. WodTKe. The Dynamics of Molecular Interactions and Chemical Reactions at Metal Surfaces: Testing the Foundations of Theory Annu. Rev. Phys. Chem., 66(1):399-425, 2015. 15
[41] H. ARNolds. Vibrational dynamics of adsorbate Quo vadis? Prog. Surf. Sci., 86(1-2):1-40, 2011. 16 18

[42] E. H. G. Backus, A. Eichler, A. W. Kleyn, and M. Bonn. Real-time observation of molecular motion on a surface Science, 310(5755):1790-1793, 2005. 17

[43] D. N.Denzler, C.Hee, S.Fund, G.Ertl, M. Bonn, C. FrischKorn, AND M. Wolf. Femtochemistry and Femtobiology: Ultrafast dynamics in molecular science chapter Surface femtochemistry: Ultrafast reaction dynamics driven by hot electron mediated reaction pathways, page 653. World Scientific, 2002. 17

[44] R. R. Chance, A. Prock, and R. Silbey. Advances in Chemical Physics 37, chapter Molecular fluorescence and energy transfer near interfaces, pages 1-65. John Wiley \& Sons, Inc., 2007. 16

[45] M. Morin, N. J. Levinos, and A. L. Harris. Vibrational energy transfer of $\mathrm{CO} / \mathrm{Cu}(100)$ : Nonadiabatic vibration/electron coupling J. Chem. Phys., 96(5):3950-3956, 1992. 17 127

[46] H. C. Chang and G. E. Ewing. Infrared fluorescence from a monolayer of $\mathrm{CO}$ on $\mathrm{NaCl}(100)$ Phys. Rev. Lett., 65(17):2125-2128, 1990. 17

[47] V. Krishna and J. C. Tully. Vibrational lifetimes of molecular adsorbates on metal surfaces J. Chem. Phys., 125(5):054706, 2006. 17

[48] M. Head-Gordon and J. C. Tully. Molecular-orbital calculations of the lifetimes of the vibrational modes of CO on Cu(100) Phys. Rev. B, 46(3):18531856, 1992. 17

[49] B. N. J. Persson and M. Persson. Vibrational lifetime for $\mathbf{C O}$ adsorbed on $\mathbf{C u ( 1 0 0 )}$ Solid State Commun., 36(2):175 - 179, 1980. 18

[50] B. D. KAY, T. D. RaYmond, and M. E. Coltrin. Observation of direct multiquantum vibrational excitation in gas-surface scattering: $\mathrm{NH}_{3}$ on $\mathrm{Au}(111)$ Phys. Rev. Lett., 59(24):2792-2794, 1987. 1819

[51] C. T. Rettner, F. Fabre, J. Kimman, and D. J. Auerbach. Observation of direct vibrational excitation in gas-surface collisions: NO on Ag(111) Phys. Rev. Lett., 55(18):1904-1907, 1985. $19[20$

[52] I. Rahinov, R. Cooper, D. Matsiev, C. Bartels, D. J. Auer$\mathrm{BACH}$, AND A. M. WODTKE, Quantifying the breakdown of the Born-Oppenheimer approximation in surface chemistry Phys. Chem. Chem. Phys., 13(28):12680-12692, 2011. 1920

[53] R. Cooper, Z. Li, K. Golibrzuch, C. Bartels, I. Rahinov, D. J. Auerbach, And A. M. WodtKe. On the determination of absolute vibrational excitation probabilities in molecule-surface scattering: Case study of NO on Au(111) J. Chem. Phys., 137(6):064705, 2012. 20

[54] K. Golibrzuch, A. Kandratsenka, I. Rahinov, R. Cooper, D. J. Auerbach, A. M. Wodtke, and C. Bartels. Experimental and theoretical study of multi-quantum vibrational excitation: $\mathrm{NO}(\mathrm{v}=\mathbf{0} \rightarrow 1,2,3)$ in Collisions with Au(111) J. Phys. Chem. A, 117(32):7091-7101, 2013. 21 
[55] Y. Huang, A. M. Wodtke, H. Hou, C. T. Rettner, and D. J. AUERBACH. Observation of vibrational excitation and deexcitation for $\mathrm{NO} v=2$ scattering from Au(111): Evidence for electron-hole-pair mediated energy transfer Phys. Rev. Lett., 84(13):2985$2988,2000.21,22$

[56] K. Golibrzuch, P. R. Shirhatti, I. Rahinov, A. KandratSenka, D. J. Auerbach, A. M. Wodtke, and C. Bartels. The importance of accurate adiabatic interaction potentials for the correct description of electronically nonadiabatic vibrational energy transfer: A combined experimental and theoretical study of $\mathrm{NO}(\mathrm{v}=3)$ collisions with a $\mathrm{Au}(111)$ surface J. Chem. Phys., 140(4):044701, 2014. 21] 22 89 120

[57] J. D. White, J. Chen, D. Matsiev, D. J. Auerbach, and A. M. WodTKe. Conversion of large-amplitude vibration to electron excitation at a metal surface Nature, 433:503-505, 2004. 23 2468,145

[58] G. KatZ, Y. ZeIRI, AND R. Kosloff. Role of vibrationally excited NO in promoting electron emission when colliding with a metal surface: A nonadiabatic dynamic model J. Phys. Chem. B, 109(40):18876-18880, 2005. 23

[59] J. LaRue, T. Schäfer, D. Matsiev, L. Velarde, N. H Nahler, D. J. Auerbach, and A. M. WodtKe. Vibrationally promoted electron emission at a metal surface: electron kinetic energy distributions Phys. Chem. Chem. Phys., 13(1):97-99, 2011. 23$] 24$ $68 \quad 145$

[60] N. H. Nahler, J. D. White, J. LaRue, D. J. Auerbach, and A. M. WODTKE. Inverse velocity dependence of vibrationally promoted electron emission from a metal surface Science, 321(5893):1191-1194, 2008. $23 \quad 24 \quad 68 \quad 145$

[61] K. Golibrzuch, P. R. Shirhatti, I. Rahinov, D. J. Auerbach, A. M. WodtKe, and C. Bartels. Incidence energy dependent state-to-state time-offlight measurements of $\mathrm{NO}(\mathrm{v}=3)$ collisions with Au(111): the fate of incidence vibrational and translational energy Phys. Chem. Chem. Phys., 16(16):7602-7610, 2014. 25

[62] B. BAULE. Theoretische Behandlung der Erscheinungen in verdünnten Gasen. Ann. Phys., 349(9):145-176, 1914. 25

63] G. Dolling, H. G. Smith, R. M. Nicklow, P. R. VijaYARAGHAVAN, AND M. K. WILKINSON. Lattice dynamics of lithium fluoride Phys. Rev., 168(3):970-979, 1968. 26

[64] A. DAL CoRso. Ab initio phonon dispersions of transition and noble metals: effects of the exchange and correlation functional $\mathrm{J}$. Phys.: Condens. Matter, 25(14):145401, 2013. 26

[65] J. W. GadzuK. Vibrational excitation in moleculesurface collisions due to temporary negative molecular ion formation J. Chem. Phys. 79(12):6341-6348, 1983. 26

[66] S. Li and H. Guo. Monte Carlo wave packet study of negative ion mediated vibrationally inelastic scattering of NO from the metal surface J. Chem. \begin{tabular}{ll|l|l|l|l|l|l|l|l|l|}
\hline Phys., 117(9):4499-4508, 2002. 27 119 & 124 & 125 \\
\hline
\end{tabular}
[67] S. Roy, N. A. Shenvi, And J. C. Tully. Model Hamiltonian for the interaction of NO with the $\mathrm{Au}(111)$ surface J. Chem. Phys., 130(17):174716, 2009. 27. 111,124

[68] N. Shenvi, S. Roy, P. Parandekar, and J. Tully. Vibrational relaxation of $\mathrm{NO}$ on $\mathrm{Au}(111)$ via electron-hole pair generation J. Chem. Phys., 125(15):154703, 2006. 27

[69] S. Monturet and P. SaAlfrank. Role of electronic friction during the scattering of vibrationally excited nitric oxide molecules from $\mathrm{Au}(111)$ Phys. Rev. B, 82(7):075404, 2010. 27] 28 [30] 124

[70] N. Shenvi, S. Roy, and J. C. Tully. Nonadiabatic dynamics at metal surfaces: Independent-electron surface hopping J. Chem. Phys., 130(17):174107, 2009. 27,124

[71] U. Heinzmann, S. Holloway, A. W. Kleyn, R. E. Palmer, AND K. J. Snowdon. Orientation in molecule-surface interactions J. Phys.: Condens. Matter, 8(19):3245, 1996. 31

[72] G.H. Fecher, N. Böwering, M. Volkmer, B. PaWlitzky, and U. Heinzmann. Dependence of the sticking probability on initial molecular orientation: NO on Ni(100) Surf. Sci., 230(1-3):L169 - L172, 1990. 31

[73] M. Brandt, H. Müller, G. Zagatta, O. Wehmeyer, N. Böwering, and U. Heinzmann. Sticking and scattering of gas-phase oriented $\mathrm{NO}$ at $\mathrm{Pt}(100)$ as a function of the translational energy and of molecular orientation Surf. Sci., 331-333(Part A):30-34, 1995. 31

[74] M. HashiNOKUChi, M. OKaDa, H. ITO, T. Kasai, K. MORITANI, AND Y. TERAOKA. Stereodynamics in dissociative adsorption of NO on $\mathrm{Si(111)}$ Phys. Rev. Lett., 100(25):256104, 2008. 31

[75] M. OKada, S. Goto, and T. Kasai. Dynamical steric effect in the decomposition of methyl chloride on a silicon surface Phys. Rev. Lett., 95(17):176103, 2005. 31

[76] H. Müller, G. Zagatta, M. Brandt, O. Wehmeyer, N. BöWERING, AND U. Heinzmann. Orientation dependent $\mathrm{CO}_{2}$ production by exposing a $\mathrm{CO}$ precovered $\mathrm{Pt}(100)$ surface to gas phase oriented NO Surf. Sci., 307-309(Part A):159-164, 1994. 31

[77] M. Brandt, G. Zagatta, N. Böwering, and U. Heinzmann. Reaction of $\mathrm{NO}$ and $\mathrm{CO}$ on a $\mathrm{Pt}(100)$ surface as a function of the NO translational energy and of molecular orientation Surf. Sci., 385(2-3):346-356, 1997. 31

[78] M. Brandt, T. Greber, N. Böwering, and U. Heinzmann. The role of molecular state and orientation in harpooning reactions: $\mathbf{N}_{\mathbf{2}} \mathrm{O}$ on $\mathbf{C s} / \mathbf{P t}(111)$ Phys. Rev. Lett., 81(11):2376-2379, 1998. 31,32

[79] H. U. Suter and T. Greber. On the dissociation of $\mathrm{N}_{\mathbf{2}} \mathrm{O}$ after electron attachment J. Phys. Chem. B, 108(38):14511-14517, 2004. 33 


\section{REFERENCES}

[80] T. FöRSTER. Zwischenmolekulare Energiewanderung und Fluoreszenz Ann. Phys., 437(1-2):55$75,1948.33$

[81] P. R. Brooks, P. W. Harland, S. A. Harris, T. Kennair, C. Redden, and J. F. Tate. Steric effects in electron transfer from potassium to $\pi$-bonded oriented molecules $\mathbf{C H}_{3} \mathbf{C N}, \mathbf{C H}_{3} \mathbf{N C}$, and $\mathbf{C C l}_{3} \mathbf{C N} \mid J A C S$, 129(50):15572-15580, 2007. 33

[82] A. W. KLEYN. Rotational rainbow scattering of oriented molecules Surf. Rev. Lett., 1(1):157-173, 1994. $33 \quad 35[37$

[83] A. W. Kleyn, A. C. Luntz, and D. J. Auerbach. Rotational energy transfer in direct inelastic surface scattering: $\mathrm{NO}$ on $\mathbf{A g}(\mathbf{1 1 1})$ Phys. Rev. Lett., 47(16):1169-1172, 1981. 33 [34

[84] F. H. Geuzebroek, A. E. Wiskerke, M. G. Tenner, A. W. Kleyn, S. Stolte, and A. NAmiki. Rotational excitation of oriented molecules as a probe of molecule-surface interaction J. Phys. Chem., 95(21):8409-8421, 1991. 35

[85] H. Voges and R. Schinke. A double rainbow interpretation of rotational energy transfer in energetic NO/Ag(111) collisions Chem. Phys. Lett., 100(3):245-250, 1983. 35

[86] J. A. Barker, A. W. Kleyn, and D. J. Auerbach. Rotational energy distributions in molecule surface scattering: Model calculations for $\mathrm{NO} / \mathrm{Ag}(111)$ Chem. Phys. Lett., 97(1):9-13, 1983. 35

[87] M. G. Tenner, F. H. Geuzebroek, E. W. Kuipers, A. E. Wiskerke, A. W. Kleyn, S. Stolte, And A. Namiki. Orientation dependence of rotational excitation in NO scattering from $\mathbf{A g ( 1 1 1 )}$ Chem. Phys. Lett., 168(1):45-50, 1990. 35113

[88] M. G. Tenner, E. W. Kuipers, A. W. Kleyn, and S. Stolte. Classical trajectory study of the interaction of oriented NO and Ag(111) Surf. Sci., 242(1-3):376$385,1991.3536$

[89] C.T. Rettner, J. Kimman, F. Fabre, D.J. Auerbach, and H. MoRaWitz. Direct vibrational excitation in gassurface collisions of NO with Ag(111) Surface Science, 192(1):107 - 130, 1987. 37

[90] A. M. WodtKe, Y. Huang, and D. J. Auerbach. Observation of orientational influences on vibrational energy transfer at metal surfaces: Rotational cooling associated with vibrational relaxation at a metal surface Chem. Phys. Lett., 414(1-3):138-142, 2005. 37

[91] G. Scole, D. Bassi, U. Buck, and D. Lainé, editors. Atomic and molecular beam methods: Volume 1. Oxford University Press, 1988. 3957

[92] L. E. Davis, N. C. MacDonald, P. W. Palmberg, G. E. RiACH, AND R. E. WEBER, editors. Handbook of Auger electron spectroscopy: Second edition. Physical Electronics Inc., 1976. 42

[93] F. Hanke And J. BJöRK. Structure and local reactivity of the $\mathbf{A u}(111)$ surface reconstruction Phys. Rev. B, 87(23):235422, 2013. 43
[94] L. Velarde, D. P. Engelhart, D. Matsiev, J. LaRue, D. J. Auerbach, and A. M. Wodtke. Generation of tunable narrow bandwidth nanosecond pulses in the deep ultraviolet for efficient optical pumping and high resolution spectroscopy Rev. Sci. Instrum., 81(6):063106, 2010. 44

[95] P. Mahnke, H.H. Klingenberg, A. Fix, and M. Wirth. Dependency of injection seeding and spectral purity of a single resonant KTP optical parametric oscillator on the phase matching condition Appl. Phys. B, 89(1):1-7, 2007. 44

[96] N. BARTELS. High-resolution spectroscopy with pulsed lasers. Master's thesis, Georg-August-Universität

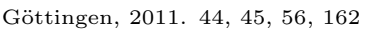

[97] Y. He, G. W. BaXter, and B. J. ORR. Locking the cavity of a pulsed periodically poled lithium niobate optical parametric oscillator to the wavelength of a continuous-wave injection seeder by an intensity-dip method Rev. Sci. Instrum., 70(8):3203-3213, 1999. 44

[98] K. H. Kramer and R. B. Bernstein. Focusing and rientation of symmetric top molecules with the electric six-pole field J. Chem. Phys., 42(2):767-770, 1965. 4955

[99] D. H. Parker and R. B. Bernstein. Oriented molecule beams via the electrostatic hexapole: Preparation, characterization, and reactive scattering Annu. Rev. Phys. Chem., 40(1):561-595, 1989. 4955

[100] R. N. ZARE. Angular momentum - understanding spatial aspects in chemistry and physics. John Wiley \& Sons, Inc., 1988. 50 155 $156157158 \quad 159$

[101] J. M. Brown and A. Carrington. Rotational spectroscopy of diatomic molecules. Cambridge University Press, 2003. 50155$] 162$

[102] S. A. MeEk. A Stark decelerator on a chip $\mathrm{PhD}$ thesis,

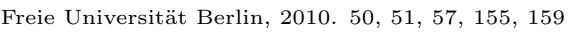

[103] M. J. L. Lange, J. J. van Leuken, M. Drabbels, J. Bulthuis, J. G. Snijders, and S. Stolte. Direct spectroscopic determination of the degree of orientation of parity selected NO Chem. Phys. Lett., 294(4-5):332-338, 1998. 52

[104] J. Danielak, U. Domin, R. Ke, M. Rytel, and M. ZachWIEJA. Reinvestigation of the emission $\gamma$-band system $\left(\mathrm{A}^{2} \boldsymbol{\Sigma}^{+}-\mathrm{X}^{2} \Pi\right)$ of the NO molecule $J$. Mol. Spec., 181(2):394-402, 1997. [52 [59]63 64 64 [173

[105] M. Drabbels and A. M. WodtKe. The determination of the infrared radiative lifetimes of a vibrationally excited neutral molecule using stimulated-emission-pumping, molecular-beam time-of-flight J. Chem. Phys., 106(8):3024-3028, 1997. 52 $63,64,68$

[106] C. VAlLANCE. Generation, characterisation, and applications of atomic and molecular alignment and orientation Phys. Chem. Chem. Phys., 13(32):1442714441, 2011. 54

107] H G. Bennewitz, W Paul and C. Schlie Fokussierung polarer Molekule Z. Phys., 141(1 2):6-15, 1955. 55 
[108] M. Drabbels, S. Stolte, and G. Meijer. Production of an intense pulsed beam of oriented metastable CO $\mathbf{a}^{3} \Pi$ Chem. Phys. Lett., 200(1-2):108-112, 1992. 56 130

[109] B. VAN de MeErakKer. Cold and controlled collisions using Stark deccelerated beams. PhysikalischChemisches Kolloquium der Universität Göttingen, 2014 57

[110] M. OKada, S. Goto, and T. Kasai. Steric effects in dissociative adsorption of low-energy $\mathrm{CH}_{3} \mathrm{Cl}$ on Si(100): Orientation and steering effects J. Phys. Chem. C, 112(49):19612-19615, 2008. 57

[111] A. Gross, S. WilKe, AND M. Scheffler. 6-dimensional quantum dynamics of adsorption and desorption of $\mathrm{H}_{2}$ at $\mathrm{Pd}(100)$ - Steering and steric effects Phys. Rev. Lett., 75(14):2718-2721, 1995. 57 117

[112] J. T. Kindt and J. C. Tully. Dynamical corrugation: simulations of the sticking of $\mathrm{CO}$ on $\mathbf{C u}(100)$ Surf. Sci., 477(2-3):149-162, 2001. 57

[113] D. O. N. Gardner, A. Al-Halabi, and G. J. Kroes. Predictions on the effect of initial molecular orientation and rotation on penetration of the (0001) face of hexagonal ice by $\mathbf{H C l}$ Chem. Phys. Lett., 376(5-6):581-587, 2003. 57

[114] H. F. Busnengo, E. Pijper, G. J. Kroes, and A. Salin. Rotational effects in dissociation of $\mathrm{H}_{2}$ on $\mathrm{Pd}(111)$ : Quantum and classical study J. Chem. Phys., 119(23):12553-12562, 2003. 57

[115] C. S. Parmenter. Vibrational-energy flow within excited electronic states of large molecules $\mathrm{J}$. Phys. Chem., 86(10):1735-1750, 1982. 67

[116] C. Kittrell, E. Abramson, J. L. Kinsey, S. A. McDonald, D. E. Reisner, R. W. Field, and D. H. Katayama. Selective vibrational excitation by Stimulated Emission Pumping J. Chem. Phys., 75(5):2056-2059, 1981. 67.128

[117] M. Silva, R. Jongma, R. W. Field, and A. M. Wodtke. The dynamics of "stretched molecules": Experimental studies of highly vibrationally excited molecules with stimulated emission pumping Annu. Rev. Phys. Chem., 52:811-852, 2001. 68 111 128

[118] M. Drabbels and A. M. WodtKe. Collisions and chemistry of super-excited molecules: Experiments using the PUMP-DUMP-PROBE technique $J$. Phys. Chem. A, 103(36):7142-7154, 1999. 68

[119] G. W. Flynn, C. S. Parmenter, and A. M. WodtKe. Vibrational energy transfer J. Phys. Chem. 100(31):12817-12838, 1996. 68

[120] R. L. Miller, A. G. Suits, P. L. Houston, R. Toumi, J. A. Mack, and A. M. WodtKe. The "ozone deficit problem"- $\mathrm{O}_{2}(\mathrm{X}, v \geq 26)+\mathrm{O}\left({ }^{3} P\right)$ from 226-nm ozone photodissociation Science, 265(5180):18311838, 1994. 68

[121] J. M. Price, J. A. Mack, C. A. Rogaski, And A. M. Wodtke. Vibrational-state-specific self-relacation rate constant measurements of highly vibrationally excited $\mathrm{O}_{\mathbf{2}}(\boldsymbol{v}=\mathbf{1 9}-\mathbf{2 8})$ Chem. Phys., 175(1):83-98, 1993. 68
[122] C. A. Rogaski, J. M. Price, J. A. Mack, and A. M. WODTKE. Laboratory evidence for a possible nonLTE mechanism of stratospheric ozone formation Geophys. Res. Lett., 20(24):2885-2888, 1993. 68

[123] F. R. Gilmore. Potential energy curves for $\mathbf{N}_{\mathbf{2}}$, NO, $\mathrm{O}_{2}$ and corresponding ions J. Quant. Spectrosc. Radiat. Transfer, 5(2):369-389, 1965. 70

[124] I. Stunart McDermid and J. B. Laudenslager. Radiative lifetimes and electronic quenching rate constants for single-photon-excited rotational levels of no $\left(\mathbf{A}^{2} \boldsymbol{\Sigma}^{+}\left(\boldsymbol{v}^{\prime}=\mathbf{0}\right)\right)$ J. Quant. Spectrosc. Radiat. Transfer, 27(5):483-492, 1982 . 70

[125] T. B. Settersten, B. D. Patterson, and W. H. Humphries, Radiative lifetimes of NO $\mathrm{A}^{2} \Sigma^{+}\left(v^{\prime}=0,1,2\right)$ and the electronic transition moment of the $\mathbf{A}^{2} \boldsymbol{\Sigma}^{+}-\mathbf{X}^{2} \Pi$ system J. Chem. Phys., 131(10): 104309, 2009. 70

[126] J. Luque and D. R. CRosley. Radiative and predissociative rates for $\mathrm{NO} \mathrm{A}^{2} \boldsymbol{\Sigma}^{+} \boldsymbol{v}^{\prime}=0-5$ and $\mathbf{D}^{\mathbf{2}} \boldsymbol{\Sigma}^{+} \boldsymbol{v}^{\prime}=\mathbf{0}-\mathbf{3}$ J. Chem. Phys., 112(21):94119416, 2000. 70

[127] J. Luque and D. R. Crosley. Transition probabilities and electronic transition moments of the $\mathrm{A}^{2} \Sigma^{+}-\mathrm{X}^{2} \Pi$ and $\mathrm{D}^{2} \boldsymbol{\Sigma}^{+}-\mathrm{X}^{2} \Pi$ systems of nitric oxide J. Chem. Phys., 111(16):7405-7415, 1999. 70

[128] N. Bartels, B. C. Krüger, S. Meyer, A. M. Wodtke, AND T. SCHÄFER. Suppression of spontaneous emission in the optical pumping of molecules: Pump-dump-sweep-probe J. Phys. Chem. Lett. 4(14):2367-2370, 2013. 727375

[129] S. Meyer. Pump-dump-sweep-probe: Improvements in stimulated emission pumping. Bachelor thesis, 2013. 72

[130] K. Bergmann, H. Theuer, and B. W. Shore. Coherent population transfer among quantum states of atoms and molecules Rev. Mod. Phys., 70(3):10031025, 1998. 74

[131] N. V. Vitanov, T. Halfmann, B. W. Shore, and K. Bergmann. Laser-induced population transfer by adiabatic passage techniques Annu. Rev. Phys. Chem., 52(1):763-809, 2001. 74

[132] P. Král, I. Thanopulos, and M. Shapiro. Colloqium: Coherently controlled adiabatic passage Rev. Mod. Phys., 79(1):53-77, 2007. 74

[133] P. Dittmann, F. P. Pesl, J. Martin, G. W. Coulston G. Z. He, and K. Bergmann. The effect of vibrational excitation $\left(3 \leq v^{\prime} \leq 19\right)$ on the reaction $\mathrm{Na}_{2}\left(\boldsymbol{v}^{\prime}\right)+\mathbf{C l} \rightarrow \mathbf{N a C l}+\mathrm{Na}^{*}$ J. Chem. Phys., 97(12):9472-9475, 1992. 77

[134] S. Kulin, B. Saubamea, E. Peik, J. Lawall, T. W. Hijmans, M. Leduc, And C. Cohen-TAnnoudj. Coherent manipulation of atomic wave packets by adiabatic transfer Phys. Rev. Lett., 78(22):4185-4188, 1997. 77 


\section{REFERENCES}

[135] M. Weitz, B. C. Young, And S. Chu. Atom manipulation based on delayed laser pulses in three- and four-level systems: Light shifts and transfer efficiencies Phys. Rev. A, 50(3):2438-2444, 1994. 77

[136] A. Kunn, G. W. Coulston, G. Z He, S. Schiemann, K. Bergmann, and W. S. Warren. Population transfer by stimulated Raman scattering with delayed pulses using spectrally broad light $J$. Chem. Phys., 96(6):4215-4223, 1992. 77

[137] T. Halfmann and K. Bergmann. Coherent population transfer and dark resonances in $\mathrm{SO}_{2}$ J. Chem. Phys., 104(18):7068-7072, 1996. 77

[138] S. Schiemann, A. Kuhn, S. Steuerwald, and K. Bergmann. Efficient coherent population transfer in NO molecules using pulsed lasers Phys. Rev. Lett., 71(22):3637-3640, 1993. 77 78 78 79 80

[139] A. Kuhn, S. Steuerwald, and K. Bergmann. Coherent population transfer in NO with pulsed lasers: the consequences of hyperfine structure, doppler broadening and electromagnetically induced absorption Eur. Phys. J. D, 1(1):57-70, 1998. 77

[140] B. C. KRÜGER. Orientation dependent multiquantum vibrational relaxation of highly vibrationally excited nitric oxide scattered from a Au(111) surface. Master's thesis, Georg-August-Universität Göttingen, 2013. 77 102 106172

[141] R. C. Hilborn. Einstein coefficients, cross sections, $f$ values, dipole moments, and all that $A m . J$. Phys., 50(11):982-986, 1982. 79

[142] C. H. Townes And A. L. Schawlow. Microwave spectroscopy. McGraw-Hill Publishing Company Ltd., 1955. 85

[143] W. L. Meerts and A. Dymanus. The hyperfine $\boldsymbol{\Lambda}-$ doubling spectrum of ${ }^{14} \mathrm{~N}^{16} \mathrm{O}$ and ${ }^{15} \mathrm{~N}^{16} \mathrm{O}$ J. Mol. Spec., 44:320-346, 1972. 85

[144] C. Amiot, R. Bacis, and G. Guelachvili. Infrared study of the $\mathrm{X}^{2} \Pi v=0,1,2$ levels of ${ }^{14} \mathrm{~N}^{16} \mathrm{O}$. Preliminary results on the $v=0,1$ levels of ${ }^{14} \mathrm{~N}^{17} \mathrm{O}$, ${ }^{14} \mathrm{~N}^{18} \mathrm{O}$, and ${ }^{15} \mathrm{~N}^{16} \mathrm{O}$ Can. J. Phys., 56(2):251-265, 1978. 85,173

[145] D. C. Jacobs, R. J. Madix, and R. N. Zare. Reduction of $1+1$ resonance enhanced MPI spectra to population distributions: Application to the NO $\mathbf{A}^{2} \boldsymbol{\Sigma}^{+}-\mathbf{X}^{2} \boldsymbol{\Pi}$ system J. Chem. Phys., 85(10):5469$5479,1986.89$

[146] D. C. JaCoBs AND R. N. ZARE. Reduction of $1+1$ resonance enhanced MPI spectra to populations and alignment factors $J$. Chem. Phys., 85(10):5457-5468, 1986. 89

[147] H. Zacharias, R. Schmiedl, And K.H. Welge. State selective step-wise photoionization of NO with mass spectroscopic ion detection Appl. Phys., 21(2):127133, 1980. 89

[148] L. T. EARLS. Intensities in ${ }^{\mathbf{2}} \boldsymbol{\Pi}_{-2}^{\mathbf{2}} \boldsymbol{\Sigma}$ transitions in diatomic molecules Phys. Rev., 48(5):423-424, 1935. 89
[149] K. Golibrzuch, P. R. Shirhatti, J. Altschäffel, I. Rahinov, D. J. Auerbach, A. M. Wodtke, and C. Bartels. State-to-state time-of-flight measurements of NO scattering from $\mathrm{Au}(111)$ : Direct observation of translation-to-vibration coupling in electronically nonadiabatic energy transfer J. Phys. Chem. $A, \mathbf{1 1 7}(36): 8750-8760,2013.89103$

[150] H. Zacharias, F. DE Rougemont, T. F. Heinz, And M. M. T. LoY. Ionization probabilities of $\mathrm{A}^{2} \Sigma^{+}\left(v^{\prime}=0,1,2\right)$ and $\mathrm{B}^{2} \Pi\left(v^{\prime}=0,1,2\right)$ states of NO J. Chem. Phys., 105(1):111-117, 1996. 99

[151] H. Rudolph, S. N. Dixit, V. McKoy, and W. M. Huo. $(1+1)$ resonant enhanced multiphoton ionization via the $\mathrm{A}^{2} \boldsymbol{\Sigma}^{+}$state of NO: Ionic rotational branching ratios and their intensity dependence J. Chem. Phys., 88(3):1516-1521, 1988. 99

152] J. D. White, J. Chen, D. Matsiev, D. J. Auerbach, and A. M. WoDTKE. Vibrationally promoted electron emission from low work-function metal surfaces J. Chem. Phys., 124(6):064702, 2006. 111,112

[153] A. J. Komrowski, H. Ternow, B. Razaznejad, B. BerenBak, J. Z. Sexton, I. Zoric, B. Kasemo, B. I. Lundqvist, S. Stolte, A. W. Kleyn, and A. C. Kummel. Dissociative adsorption of NO upon $\mathrm{Al}(111)$ : Orientation dependent charge transfer and chemisorption reaction dynamics J. Chem. Phys., 117(18):8185-8189, 2002. 111

154] D. Skouteris, D. E. Manolopoulos, W. Bian, H. Werner, L. LAI, AND K. LIU. Van der Waals interactions in the Cl + HD reaction Science, 286(5445):17131716, 1999. 117

[155] K.D. Rendulic, G. Anger, and A. Winkler. Wide range nozzle beam adsorption data for the systems $\mathbf{H}_{\mathbf{2}} /$ nickel and $\mathbf{H}_{2} / \mathbf{P d}(\mathbf{1 0 0})$ Surf. Sci., 208(3):404424, 1989. 117

[156] A. Gross And M. Scheffler. Ab initio quantum and molecular dynamics of the dissociative adsorption of hydrogen on Pd(100) Phys. Rev. B, 57(4):2493-2506, 1998. 117

[157] A. M. WodtKe, Y. Huang, and D. J. Auerbach. Insensitivity of trapping at surfaces to molecular vibration Chem. Phys. Lett., 413(4-6):326-330, 2005. 118 119

[158] C. T. Rettner, D. J. Auerbach, and H. A. Michelsen. Observation of direct vibrational excitation in collisions of $\mathbf{H}_{2}$ and $\mathrm{D}_{2}$ with a $\mathrm{Cu}(111)$ surface Phys. Rev. Lett., 68(16):2547-2550, 1992. 119

[159] H. Falsig, J. Shen, T. Khan, W. Guo, G. Jones, S. Daht, AND T. BLIGAARD. On the structure sensitivity of direct NO decomposition over low-index transition metal facets Top. Catal., 57(1-4):80-88, 2014. 119

[160] D. P. Engelhart, R. J. V. Wagner, A. Meling, A. M. WODTKE, AND T. SCHÄFER. Temperature programmed desorption of weakly bound adsorbates on Au(111). Manuscript in preparation, 2015. 119

[161] E. J. Salumbides, A. Khramov, and W. Ubachs. Highresolution $2+1$ REMPI study of the $a^{\prime \prime} \Sigma_{g}^{+}$ State in $\mathbf{N}_{\mathbf{2}}$ J. Phys. Chem. A, 113(11):2383-2386, 2009. 119 
[162] J. Altschäffel. Ab initio molekulardynamische Rechnungen der NO Streuung an Au(111). Master's thesis, Georg-August-Universität Göttingen, 2015. 124145

[163] M. Head-Gordon and J. C. Tully. Vibrational relaxation on metal surfaces: Molecular-orbital theory and application to $\mathbf{C O} / \mathbf{C u}(\mathbf{1 0 0}) \mathrm{J}$. Chem. Phys., 96(5):3939-3949, 1992. 127

[164] D. M. Neumark, A. M. Wodtke, G. N. Robinson, C. C. HayDEN, AND Y. T. LEE. Molecular beam studies of the $\mathbf{F}+\mathbf{H}_{\mathbf{2}}$ reaction J. Chem. Phys., 82(7):3045-3066, 1985. 128

[165] C. T. Rettner and D. J. Auerbach. Distinguishing the direct and indirect products of a gas-surface reaction Science, 263(5145):365-367, 1994. 128

[166] R. D. Beck, P. Maroni, D. C. Papageorgopoulos, T. T. DANG, M. P. SCHMid, AND T. R. Rizzo. Vibrational mode-specific reaction of methane on a nickel surface Science, 302(5642):98-100, 2003. 128

[167] L. Schnieder, K. Seekamp-Rahn, F. Liedeker, H. Steuwe, AND K. H. WeLGe. Hydrogen exchange reaction $\mathbf{H}+$ $\mathbf{D}_{2}$ in crossed beams Faraday Discuss. Chem. Soc., 91:259, 1991. 128

[168] R. W. Field And H. L. DaI, editors. Molecular dynamics and spectroscopy by stimulated emission pumping, 4. World Scientific Pub Co Inc, 1995. 128

[169] S. Wurm, P. Feulner, and D. Menzel. Extremely high vibrational excitation of $\mathrm{CO}$ molecules desorbed from transition metal surfaces by electron impact Phys. Rev. Lett., 74(13):2591-2594, 1995. 128

[170] H. C. Chang and G. E. Ewing. Vibrational energy transfer and population inversion in carbon monoxide overlayers on sodium chloride (100) $\mathrm{J}$. Phys. Chem., 94(19):7635-7641, 1990. 128

[171] C. Flament, T. George, K. A. Meister, J. C. Tufts, J. W Rich, V. V. Subramaniam, J. P. Martin, B. Piar, and M. Y. PERRIN. Nonequlibrium vibrational kinetics of carbon-monoxide at high translational mode temperatures Chem. Phys., 163(2):241-262, 1992. 128

[172] J. D. Simmons, A. M. Bass, and S. G. Tilford. Fourth positive system of carbon monoxide observed in absorption at high resolution in vacuum ultraviolet region Astrophys. J., 155(1):345, 1969. 128

[173] M. Eidelsberg, F. Rostas, J. Breton, and B. Thieblemont. Vibrational band oscillator-strengths and dipole transition-moment of the $\mathbf{A}^{\mathbf{1}} \Pi-\mathbf{X}^{1} \boldsymbol{\Sigma}^{+}$system of CO J. Chem. Phys., 96(8):5585-5590, 1992. 128

[174] R. W. Field, S. G. Tilford, J. D. Simmons, and R. A. HOWARD. Fine-structure and perturbation analy-

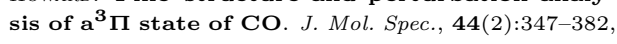
1972. 128 $1 3 0 \longdiv { 1 3 2 }$

[175] R. W. Field and H. Lefebrre-Brion. The spectra and $d y-$ namics of diatomic molecules. Academic Press; 1 edition, 2004. $130161 \quad 162163164$
[176] J. M. Price, A. Ludviksson, M. Nooney, M. Xu, R. M. Martin, and A. M. Wodtke. Time-of-flight measurements of single rovibrational states of carbonmonoxide J. Chem. Phys., 96(3):1854-1857, 1992. 130

[177] J.T. Hougen. The Calculation of Rotational Energy Levels and Rotational Line Intensities in Diatomic Molecules (version 1.0) [Online] Available: http://physics.nist.gov/DiatomicCalculations 2001. 132

[178] P. H. KRupenie. The band spectrum of carbon monoxide National Standard Reference Data Series, 1966. 132

[179] F. Rostas, M. Eidelsberg, A. Jolly, J. L. Lemaire, A. LE FLOCH, AND J. Rostas. Band oscillator strengths of the intersystem transitions of CO J. Chem. Phys., 112(10):4591-4603, 2000. 132 166

[180] M. Eidelsberg and F. Rostas. An atlas of the intersystem transitions of CO ApJS, 145(1):89-109, 2003. 132

[181] A. MeLing. Preparation of velocity controlled molecular beams of highly vibrationally excited CO. Master's thesis, Georg-August-Universität Göttingen, 2015. 135 141

[182] D. C. Morton and L. Noreau. A compilation of electronic transitions in the $\mathrm{CO}$ molecule and the interpretation of some puzzling interstellar absorption features $A p J S$, 95(1):301-343, 1994. 136

[183] R. W. FIELD. Spectroscopy and perturbation analysis in excited states of $C O$ and $C S$ (and supplementary material). PhD thesis, Harvard University, 1972. 136140 167

[184] A. Lefloch, J. Rostas, and F. Rostas. Lifetimes of the

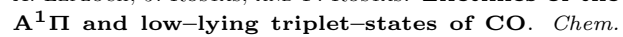
Phys., 142(2):261-278, 1990. 137

[185] J. H. Blokland, J. Riedel, S. Putzke, B. G. Sartakov, G. C. Groenenboom, and G. Meijer. Producing translationally cold, ground-state CO molecules $J$. Chem. Phys., 135(11):114201, 2011. 139165

[186] R. L. RoY. RKR1 2.0, A computer program implementing the first-order RKR method for determining diatomic molecule potential energy functions. University of Waterloo, (CP657R), 2004. 140

[187] R. L. RoY. LEVEL 8.0, A computer program for solving the radial Schrödinger equation for bound and quasibound levels. University of Waterloo, (CP-663), 2007. 140

[188] A. C. Le Floch. PhD thesis, Univ. Paris-Sud, 1989. 168

[189] J. Luque and D. R. CRosley. LifBASE: Database and Spectral Simulation Program (Version 1.6). SRI International Report, (MP-99-009), 1991. 173

[190] С. Аміот. The infrared emission spectrum of NO: Analysis of the $\Delta \boldsymbol{v}=\mathbf{3}$ sequence up to $\boldsymbol{v}=\mathbf{2 2}$ J. Mol. Spec., 94(1):150-172, 1982. 173 


\section{Appendix A}

\section{Basics of angular momentum coupling and an effective Hamiltonian for diatomic molecules}

This section reviews some theory concerning angular momentum theory (anuglar momentum operators, Clebsch-Gordan coefficients, 3-j symbols, and Euler angles, the derivation of the a rigid rotor wavefunction) and an effective Hamiltonian for diatomic molecules. It mostly follows the book of Brown and Carrington (101), but also of Zare (100) and the PhD thesis of Samuel Meek (102).

\section{A.1 Angular momentum operators}

In classical mechanics, a particle of mass $m$ and velocity $\mathbf{v}$ has a linear momentum of

$$
\mathbf{p}=m \mathbf{v}
$$

and an angular momentum of $\mathbf{l}$ given by

$$
\mathbf{l}=\mathbf{r} \times \mathbf{p} .
$$

The conversion to quantum mechanics is achieved by replacing $\mathbf{p}$ by $(\hbar / i) \nabla$ with the Nabla operator $\nabla$ given by

$$
\nabla=\frac{\partial}{\partial x} \hat{\mathbf{x}}+\frac{\partial}{\partial y} \hat{\mathbf{y}}+\frac{\partial}{\partial z} \hat{\mathbf{z}} .
$$




\section{A. Basics of angular momentum coupling and an effective Hamiltonian for diatomic molecules}

The commutation relation between the Cartesian components of 1 are given by ((100), page 2)

$$
\left[l_{x}, l_{y}\right]=i l_{z}, \quad\left[l_{y}, l_{z}\right]=i l_{x}, \quad\left[l_{z}, l_{x}\right]=i l_{y}
$$

This means that not all of the components of the angular momentum can be measured exactly at the time.

Often, a general angular momentum operator $\mathbf{j}$ is introduced, which obeys the same commutation rules (later the label $\mathbf{l}$ will exclusively be used for the orbital angular momentum of the electrons):

$$
\left[j_{x}, j_{y}\right]=i j_{z}, \quad\left[j_{y}, j_{z}\right]=i j_{x}, \quad\left[j_{z}, j_{x}\right]=i j_{y}
$$

We further define the square of the angular momentum operator by

$$
\mathbf{j}^{2}=j_{x}^{2}+j_{y}^{2}+j_{z}^{2}
$$

which has the following commutation properties:

$$
\left[\mathbf{j}^{2}, j_{x}\right]=\left[\mathbf{j}^{2}, j_{y}\right]=\left[\mathbf{j}^{2}, j_{z}\right]=0
$$

Thus we can construct states $|j m\rangle$ that are simultaneous eigenfunctions of $\mathbf{j}^{2}$ and a component of $\mathbf{j}$ for which we choose $j_{z}$. These obey the eigenvalue equations ((100), page 3)

$$
\begin{aligned}
\left\langle j m\left|\mathbf{j}^{2}\right| j^{\prime} m^{\prime}\right\rangle & =j(j+1) \delta_{j, j^{\prime}} \delta_{m, m^{\prime}} \\
\left\langle j m\left|j_{z}\right| j^{\prime} m^{\prime}\right\rangle & =m^{\prime} \delta_{j, j^{\prime}} \delta_{m, m^{\prime}},
\end{aligned}
$$

where $m$ can take successive values between $m=-j$ and $m=j$ that differ by unity. $\delta_{m, m^{\prime}}$ is the Kronecker delta with the properties that $\delta_{m, m^{\prime}}=1$ for $m=m^{\prime}$ and $\delta_{m, m^{\prime}}=0$ for $m \neq m^{\prime}$.

We further define the raising $j_{+}$and lowering $j_{-}$operators

$$
j_{+}=j_{x}+i j_{y}, \quad j_{-}=j_{x}-i j_{y},
$$

which give the off-diagonal matrix elements

$$
\left\langle j m\left|j_{ \pm}\right| j^{\prime} m^{\prime}\right\rangle=\left[j(j+1)-m^{\prime}\left(m^{\prime} \pm 1\right)\right]^{\frac{1}{2}} \delta_{j, j^{\prime}} \delta_{m, m^{\prime} \pm 1} .
$$




\section{A.2 Coupling of two angular momentum vectors: Clebsch- Gordan Coefficients and 3j-Symbols}

Most problems in spectroscopy involve at least two different angular momenta $\mathbf{j}_{1}$ and $\mathbf{j}_{2}$ that compose the total angular momentum $\mathbf{j}$

$$
\mathbf{j}=\mathbf{j}_{1}+\mathbf{j}_{2}
$$

It is easy to show, that the sum of two angular momenta is also an angular momentum in a sense, that it obeys the commutation rules as given in equation A.5.

A system with two angular momenta can be described in two different useful ways.((100), page 43) The first way is a description using the complete set of commuting angular momentum operators $\mathbf{j}_{1}^{2}, j_{1 z}, \mathbf{j}_{2}^{2}$ and $j_{2 z}$. Here, the states $\left|j_{1} m_{1}, j_{2} m_{2}\right\rangle \equiv$ $\left|j_{1} m_{1}\right\rangle\left|j_{2} m_{2}\right\rangle$ are eigenfunctions of these operators

$$
\begin{aligned}
\mathbf{j}_{1}^{2}\left|j_{1} m_{1}, j_{2} m_{2}\right\rangle & =j_{1}\left(j_{1}+1\right)\left|j_{1} m_{1}, j_{2} m_{2}\right\rangle \\
j_{1 z}\left|j_{1} m_{1}, j_{2} m_{2}\right\rangle & =m_{1}\left|j_{1} m_{1}, j_{2} m_{2}\right\rangle \\
\mathbf{j}_{2}^{2}\left|j_{1} m_{1}, j_{2} m_{2}\right\rangle & =j_{2}\left(j_{2}+1\right)\left|j_{1} m_{1}, j_{2} m_{2}\right\rangle \\
j_{2 z}\left|j_{1} m_{1}, j_{2} m_{2}\right\rangle & =m_{2}\left|j_{1} m_{1}, j_{2} m_{2}\right\rangle
\end{aligned}
$$

The $\left|j_{1} m_{1}, j_{2} m_{2}\right\rangle$ states span a space of dimension $\left(2 j_{1}+1\right)\left(2 j_{2}+1\right)$ and are called the uncoupled representation.

Another representation called the coupled representation of dimension $2 j+1$ for each $J$ value uses the commuting angular momentum operators $\mathbf{j}_{1}^{2}, \mathbf{j}_{2}^{2}, \mathbf{j}^{2}=\left(\mathbf{j}_{1}+\mathbf{j}_{2}\right)^{2}$ and $j_{z}=j_{1 z}+j_{2 z}$. In that case the eigenvalue equations with the eigenfunctions $\left|j_{1} j_{2} j m\right\rangle$ — often abbreviated as $|j m\rangle$ - are

$$
\begin{aligned}
\mathbf{j}_{1}^{2}|j m\rangle & =j_{1}\left(j_{1}+1\right)|j m\rangle \\
\mathbf{j}_{2}^{2}|j m\rangle & =j_{2}\left(j_{2}+1\right)|j m\rangle \\
\mathbf{j}^{2}|j m\rangle & =j(j+1)|j m\rangle \\
j_{z}|j m\rangle & =m|j m\rangle
\end{aligned}
$$

These two representations are connected via the unitary transformation

$$
|j m\rangle=\sum_{m_{1}, m_{2}} C\left(j_{1} j_{2} j ; m_{1} m_{2} m\right)\left|j_{1} m_{1}, j_{2} m_{2}\right\rangle
$$




\section{A. Basics of angular momentum coupling and an effective Hamiltonian for diatomic molecules}

and the inverse transformation

$$
\left|j_{1} m_{1}, j_{2} m_{2}\right\rangle=\sum_{j, m} C\left(j_{1} j_{2} j ; m_{1} m_{2} m\right)|j m\rangle
$$

respectively, where the elements of the transformation

$$
C\left(j_{1} j_{2} j ; m_{1} m_{2} m\right) \equiv\left\langle j_{1} m_{1}, j_{2} m_{2}|| j m\right\rangle \equiv\left\langle j m|| j_{1} m_{1}, j_{2} m_{2}\right\rangle
$$

are the Clebsch-Gordan coefficients, which are chosen to be real. The Clebsch-Gordan coefficients vanish unless the relations $m=m_{1}+m_{2}$ (so-called triangulation relation) and $\left|j_{1}+j_{2}\right|=j=\left|j_{1}-j_{2}\right|$ is satisfied. In general, the angular momenta $\mathbf{j}_{1}$ and $\mathbf{j}_{2}$ add vectorially, while their projections (magnetic quantum numbers) $m_{1}$ and $m_{2}$ add algebraically. Clebsch-Gordan coefficients are tabulated in many textbooks. The easiest way to obtain them with modern computer software such as Wolfram Mathematica however is to let the software calculate Wigner $3-j$ symbols (the command is ThreeJSymbol). The Wigner $3-j$ symbols are connected to the Clebsch-Gordan coefficients via $((100)$, page 50$)$

$$
\begin{aligned}
\left(\begin{array}{ccc}
j_{1} & j_{2} & j_{3} \\
m_{1} & m_{2} & m_{3}
\end{array}\right) & \equiv(-1)^{j_{1}-j_{2}-m_{3}}\left(2 j_{3}+1\right)^{-\frac{1}{2}}\left\langle j_{1} m_{1}, j_{2} m_{2} \mid j_{3}-m_{3}\right\rangle \\
\left\langle j_{1} m_{1}, j_{2} m_{2} \mid j_{3} m_{3}\right\rangle & \equiv(-1)^{j_{1}-j_{2}+m_{3}}\left(2 j_{3}+1\right)^{\frac{1}{2}}\left(\begin{array}{ccc}
j_{1} & j_{2} & j_{3} \\
m_{1} & m_{2} & -m_{3}
\end{array}\right) .
\end{aligned}
$$

\section{A.3 Transformation under rotation}

An arbitrary rotation of two coordinate systems with respect to each other can be described using three angles. In spectroscopy, we often want to relate space fixed coordinates $(X, Y$ and $Z)$ to molecule fixed coordinates $(x, y$, and $z)$. Starting from a reference orientation with $x, y$ and $z$ coincident with $X, Y$ and $Z$, the molecule fixed coordinates are rotated with the following three rotations ((100), pp. 45-46):

1. a counter-clockwise rotation about the initial $Z$-axis through an initial angle $\phi$ $(0<\phi \leq 2 \pi)$

2. a subsequent rotation about the resultant $Y$-axis through an angle $\theta(0<\theta \leq \pi)$

3. a final rotation about the resultant $Z$-axis through an angle $\chi(0<\chi \leq 2 \pi)$ 
Here the angles $\chi, \theta$ and $\phi$ are called the Euler angles.

It can be shown, that the full transformation between space fixed and molecule fixed coordinate systems

$$
\left(\begin{array}{l}
x \\
y \\
z
\end{array}\right)=R\left(\begin{array}{l}
X \\
Y \\
Z
\end{array}\right)
$$

is obtained by the direction cosine matrix $R$ given by ((100), p. 81)

$$
R=\left(\begin{array}{ccc}
\cos \phi \cos \theta \cos \chi-\sin \phi \sin \chi & \sin \phi \cos \theta \cos \chi+\cos \phi \sin \chi & -\sin \theta \cos \chi \\
-\cos \phi \cos \theta \sin \chi-\sin \phi \cos \chi & -\sin \phi \cos \theta \sin \chi+\cos \phi \cos \chi & \sin \theta \sin \chi \\
\cos \phi \sin \theta & \sin \phi \sin \theta & \cos \theta
\end{array}\right)
$$

How does a nuclear momentum eigenstate $|J M\rangle$ behave under rotation? Obviously, a rotation does not change the total angular momentum $J$, but only the projection $M$ on the space fixed axis. Thus, the rotation $\mathbf{R}(\phi, \theta, \chi)$ transforms $|J M\rangle$ into a linear combination of other $M$ values.

$$
\mathbf{R}(\phi, \theta, \chi)|J M\rangle=\sum_{M^{\prime}} D_{M^{\prime} M}^{J}(\phi, \theta, \chi)\left|J M^{\prime}\right\rangle
$$

where $D_{M M^{\prime}}^{J}(\phi, \theta, \chi)$ are the elements of a $(2 J+1) \times(2 J+1)$ matrix called Wigner rotation matrix. Often books present the elements of that matrix in the form of a reduced rotation matrix $d_{M M^{\prime}}^{J}(\phi, \theta, \chi)$, which is related by the equation

$$
D_{M M^{\prime}}^{J}(\phi, \theta, \chi)=\exp \left(-i \phi M^{\prime}-i \chi m\right) d_{M M^{\prime}}^{J}(\phi, \theta, \chi)
$$

In Wolfram Mathematica the elements $D_{M^{\prime} M}^{J}$ are obtained via the command WignerD.

\section{A.4 Wave function of a rigid rotor}

In the molecular frame, the Hamiltonian of a rigid rotor with rotational constants $(A \neq B=C)$ is given by $((\underline{102})$, page 44$)$

$$
\hat{H}=A J_{z}^{2}+B J_{y}^{2}+C J_{x}^{2}
$$

Using that $\mathbf{J}^{2}=J_{x}^{2}+J_{y}^{2}+J_{z}^{2}$, this expression can be rewritten as

$$
\hat{H}=B \mathbf{J}^{2}+(A-B) J_{z}^{2} .
$$




\section{A. Basics of angular momentum coupling and an effective Hamiltonian for diatomic molecules}

The molecule fixed angular momentum operator $J_{z}$ commutes with the space fixed angular momentum operator $J_{Z}$. Thus, we use the angular momentum wave function $|J M\rangle$, which is an eigenstate of $\mathbf{J}^{2}$ (eigenvalue $J(J+1)$ ) and of $J_{Z}$ (eigenvalue of $\mathrm{M}$ ). A rotation around the Euler angles $(\phi, \theta, \chi)$ is equivalent to rotating the coordinate system around $(-\chi,-\theta,-\phi)$ and vice versa. Thus, a wave function at a position $\mathrm{s}$ $(\phi, \theta, \chi)$ can be evaluated by rotating it through the Euler angles $(-\chi,-\theta,-\phi)$ and then evaluating the transformed function at $\theta=\phi=\chi=0$. Combining this fact with the definition of the Wigner D matrix, the wavefunction can be expressed as

$$
|J M\rangle(\phi, \theta, \chi)=\mathbf{R}(-\chi,-\theta,-\phi)|J M\rangle(0,0,0)
$$

Using equation A.20 this can be rewritten as (writing $\Omega^{\prime}$ for $M^{\prime}$ )

$$
|J M\rangle(\phi, \theta, \chi)=\sum_{\Omega^{\prime}}\left|J \Omega^{\prime}\right\rangle(0,0,0) D_{\Omega^{\prime} M}^{J}(-\chi,-\theta,-\phi) .
$$

The rotation matrix has the symmetry property

$$
D_{M^{\prime} M}^{J}(-\chi,-\theta,-\phi)=(-1)^{M^{\prime}-M} D_{-M^{\prime}-M}^{J}(\phi, \theta, \chi)
$$

and thus we obtain

$$
|J M\rangle(\phi, \theta, \chi)=\sum_{\Omega^{\prime}}\left|J \Omega^{\prime}\right\rangle(0,0,0)(-1)^{\Omega^{\prime}-M} D_{-\Omega^{\prime}-M}^{J}(\phi, \theta, \chi) .
$$

This expression can be further simplified, as $J_{z}$ commutes with the Hamiltonian with the eigenvalue $\Omega$

$$
J_{z} D_{-\Omega^{\prime}-M}^{J}(\phi, \theta, \chi)=\Omega^{\prime} D_{-\Omega^{\prime}-M}^{J}(\phi, \theta, \chi)
$$

and thus $\left|J \Omega^{\prime}\right\rangle(0,0,0)$ is only non-zero for $\Omega^{\prime}=\Omega$, which reduces the sum $\sum_{\Omega^{\prime}}$ to a single element. By normalizing the wave function, we finally obtain an expression for the wave function of the rigid rotor

$$
|J \Omega M\rangle=(-1)^{\Omega-M}\left[\frac{2 J+1}{8 \pi^{2}}\right]^{\frac{1}{2}} D_{-\Omega-M}^{J} .
$$


Historically, this result has first been obtained by solving the set of differential equations the wave functions satisfy

$$
\begin{aligned}
J_{Z}|J \Omega M\rangle & =-i \frac{\partial}{\partial \phi}|J \Omega M\rangle=M|J \Omega M\rangle \\
J_{z}|J \Omega M\rangle & =-i \frac{\partial}{\partial \chi}|J \Omega M\rangle=\Omega|J \Omega M\rangle \\
\mathbf{J}^{2}|J \Omega M\rangle & =\left[\frac{\partial^{2}}{\partial \theta^{2}}-\cot \theta \frac{\partial}{\partial \theta}-\frac{1}{\sin ^{2} \theta}\left(\frac{\partial^{2}}{\partial \phi^{2}}+\frac{\partial^{2}}{\partial \chi^{2}}-2 \cos \theta \frac{\partial^{2}}{\partial \phi \partial \chi}\right)\right]|J \Omega M\rangle \\
& =J(J+1)|J \Omega M\rangle
\end{aligned}
$$

subject to the boundary conditions, that $\chi$ is arbitrarily fixed to $\chi=0$ and that the wave function is normalized.

\section{A.5 Hund's case a) basis functions}

The wavefunctions of a rigid rotor are important in the spectroscopy of diatomic molecules and are used in the Hund's case a) basis functions. A diatomic molecule can have several angular momenta, which are given in the following list ((175), page 72). Here, $\mathbf{l}_{i}$ is the orbital angular momentum of the $i$-th electron, $\mathbf{s}_{i}$ is the spin of the $i$ th electron and $\mathbf{R}$ is the rotation of the nuclear framework. The nuclear spin is neglected, as the hyperfine splitting is not resolved in this work.

- total electronic angular momentum $\mathbf{L}=\sum_{i} \mathbf{l}_{i}$

- total electron spin $\mathbf{S}=\sum_{i} \mathbf{s}_{i}$

- total angular momentum $\mathbf{J}=\mathbf{R}+\mathbf{L}+\mathbf{S}$

- total angular momentum excluding electronic spin $\mathbf{N}=\mathbf{J}-\mathbf{S}$

- nuclear rotational angular momentum $\mathbf{R}=\mathbf{N}-\mathbf{L}$

The Hamiltonians for diatomic molecules are often evaluated in Hund's case (a) basis functions. In Hund's case (a) (Fig. A.1), $\mathbf{L}$ and $\mathbf{S}$ both precess about the internuclear $z$-axis with the projections $\Lambda$ and $\Sigma$. The nuclear rotation angular momentum $\mathbf{R}$ is directed perpendicular to the internuclear axis. Thus, the total angular momentum $\mathbf{J}$ makes the projection $\Omega=\Lambda+\Sigma$ on the $z$-axis and the projection $M$ on the space fixed 


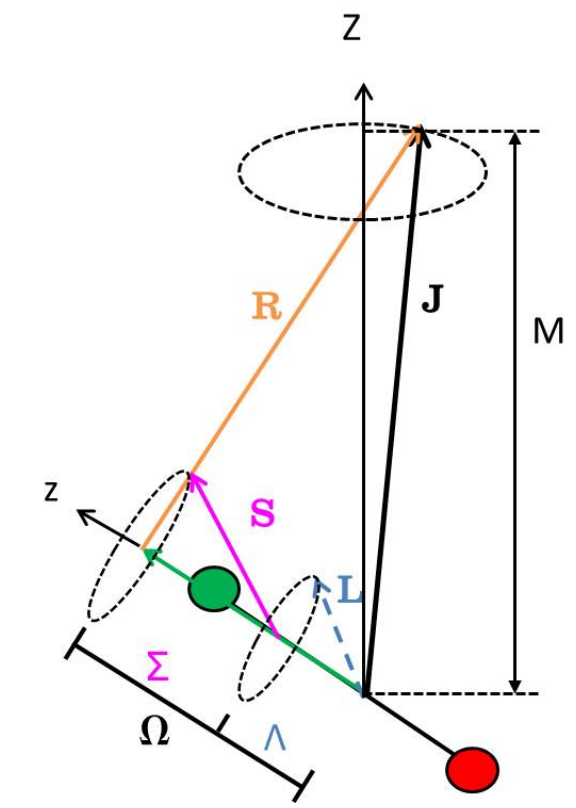

Figure A.1: Hunds case a) couping case - The figure is described in the text. Figure taken from my Masters thesis (96).

$Z$-axis.

The Hund's case (a) wave function can be written as a product of an electronic orbital part $|n \Lambda\rangle$, an electronic spin part $|S \Sigma\rangle$, a vibrational part $|v\rangle$, and a rotational part $|J \Omega M\rangle$.

$$
\left|\psi\left(n^{2 S+1} \Lambda_{\Omega} ; v J M\right)\right\rangle=|n \Lambda\rangle|S \Sigma\rangle|v\rangle|J \Omega M\rangle
$$

The rotational part of the Hund's case a) wavefunction is given as in Eq. A.29.

\section{A.6 Effective Hamiltonian for diatomic molecules}

The spectroscopy of diatomic molecules often uses the concept of an effective Hamiltonian ((101), chapter 7$)$ that only operates within a single vibrational and electronic state. The Hamiltonian used by Field et al. (175) is given by

$$
\hat{H}=\hat{H}_{e v}+\hat{H}_{\mathrm{SO}}+\hat{H}_{\mathrm{rot}}+\hat{H}_{\mathrm{SS}}+\hat{H}_{\mathrm{SR}},
$$

where $\hat{H}_{e v}$ is the vibronic part of the Hamiltonian. $\hat{H}_{\mathrm{SO}}, \hat{H}_{\mathrm{SS}}$, and $\hat{H}_{\mathrm{SR}}$ are the spinorbit, spin-spin and spin-rotation operators, respectively. Derivations for these operators and how they act on Hund's case a) wavefunctions can be found in the literature, 


\section{A.6 Effective Hamiltonian for diatomic molecules}

and here only the results are given.

The Rotational Hamiltonian is simply giving the energy of two nuclei rotating around each other ((175), page 96$)$

$$
\hat{H}_{\text {rot }}=B \mathbf{R}^{2}=\left(1 / 2 \mu R^{2}\right)\left(R_{x}^{2}+R_{y}^{2}\right),
$$

where we have used, that the rotation is perpendicular to the $z$-direction and thus $R_{z}=0$. Using that $\mathbf{R}=\mathbf{N}-\mathbf{L}=\mathbf{J}-\mathbf{L}-\mathbf{S}, \hat{H}_{\text {rot }}$ can be rewritten to the form

$$
\begin{aligned}
\hat{H}_{\mathrm{rot}}= & \left(1 / 2 \mu R^{2}\right)\left[\left(J_{x}-L_{x}-S_{x}\right)^{2}+\left(J_{y}-L_{y}-S_{y}\right)^{2}\right] \\
= & \left(1 / 2 \mu R^{2}\right)\left[\left(\mathbf{J}^{2}-J_{z}^{2}\right)+\left(\mathbf{L}^{2}-L_{z}^{2}\right)+\left(\mathbf{S}^{2}-S_{z}^{2}\right)\right. \\
& \left.+\left(L^{+} S^{-}+L^{-} S^{+}\right)-\left(J^{+} L^{-}+J^{-} L^{+}\right)-\left(J^{+} S^{-}+J^{-} S^{+}\right)\right] .
\end{aligned}
$$

The first three terms of $\hat{H}_{\text {rot }}$ have diagonal matrix elements only. This means, we can write an eigenvalue equation of the form

$$
B\left[\left(\mathbf{J}^{2}-J_{z}^{2}\right)+\left(\mathbf{S}^{2}-S_{z}^{2}\right)+\left(\mathbf{L}^{2}-L_{z}^{2}\right)\right]|J \Omega M\rangle=E_{\mathrm{rot}}(R)|J \Omega M\rangle
$$

with the eigenvalues

$$
E_{\text {rot }}(R)=\left(\hbar^{2} / 2 \mu R^{2}\right)\left[J(J+1)-\Omega^{2}+S(S+1)-\Sigma^{2}+L(L+1)-\Lambda^{2}\right] .
$$

Usually, the term $\left(\hbar^{2} / 2 \mu R^{2}\right)\left[L(L+1)-\Lambda^{2}\right]$ is incorporated to the electronic energy. Thus we obtain:

$$
E_{R o t}(v, J)=B_{v}\left[J(J+1)-\Omega^{2}+S(S+1)-\Sigma^{2}\right]
$$

The remaining three terms of the rotational operator couple the orbital, spin and total angular momenta and and are responsible for perturbations between different electronic states.((175), page 98$)$

1. $\left(1 / 2 \mu R^{2}\right) L^{ \pm} S^{\mp}$ causes homogeneous $(\Delta \Omega=0)$ spin-electronic perturbations between basis functions of the same $\Omega$ and $S$, but different $\Lambda$ and $\Sigma$.

2. $-\left(1 / 2 \mu R^{2}\right) J^{ \pm} S^{\mp}$ is called the $\mathbf{S}$ - uncoupling operator. It gives rise to heterogeneous $(\Delta \Omega \pm 1)$ electronic-rotational perturbations with the same $S$ and $\Lambda$, but different $\Sigma$. 
3. $-\left(1 / 2 \mu R^{2}\right) J^{ \pm} L^{\mp}$ is called the $\mathbf{L}$-uncoupling operator and causes heterogeneous $(\Delta \Omega \pm 1)$ electronic-rotational perturbations between states of the same $S$ and $\Sigma$, but different $\Lambda$ and $\Omega$.

For this work, the S-uncoupling operator will become relevant. This operator has the matrix elements $((\underline{175})$, table on page 97$)$

$$
\left\langle\Lambda, S, \Sigma, J, \Omega\left|-B_{v} J^{ \pm} S^{\mp}\right| \Lambda, S, \Sigma \pm 1, J, \Omega \pm 1\right\rangle=-B_{v} \sqrt{S(S+1) \cdot J(J+1)}
$$

The diagonal Matrix elements of the spin-orbit Hamiltonian $\hat{H}_{\text {SO }}$ (diagonal in all quantum numbers) that splits the energy a system into $2 S+1$ components if $\Lambda \neq 0$ is given by ((175), page 183$)$

$$
\left\langle\Lambda, \Sigma, S, \Omega, v\left|\hat{H}_{\mathrm{SO}}\right| \Lambda, \Sigma, S, \Omega, v\right\rangle=A_{\Lambda, v} \Lambda \Sigma
$$

where $A_{\Lambda, v}$ is the spin-orbit coupling constant.

This spin-spin Hamiltonian $\hat{H}_{\mathrm{SS}}$ is diagonal in the Hunds case a) basis set and has the non-zero matrix elements ((175), page 196)

$$
\left\langle S, \Sigma\left|\hat{H}_{\mathrm{SS}}\right| S, \Sigma\right\rangle=\frac{2}{3} \lambda\left[3 \Sigma^{2}-S(S+1)\right]
$$

Finally, the spin-rotation Hamiltonian $\hat{H}_{\mathrm{SR}}$ is a contribution accounting for the interaction between the electron spins and the magnetic field created by nuclear motion and has the diagonal matrix elements ((175), page 193)

$$
\left\langle\Lambda, S, \Sigma, J, \Omega, M\left|\hat{H}_{\mathrm{SR}}\right| \Lambda, S, \Sigma, J, \Omega, M\right\rangle=\gamma\left[\Sigma^{2}-S(S+1)\right]
$$

and the $\Delta \Omega=\Delta \Sigma= \pm 1, \Delta \Lambda=0, \Delta S=0$ off-diagonal elements,

$$
\begin{aligned}
& \left\langle\Lambda, S, \Sigma, J, \Omega, M\left|\hat{H}_{\mathrm{SR}}\right| \Lambda, S, \Sigma \pm 1, J, \Omega \pm 1, M\right\rangle= \\
& \gamma_{v} / 2[J(J+1)-\Omega(\Omega \pm 1)]^{0.5}[S(S+1)-\Sigma(\Sigma+1)]^{0.5}
\end{aligned}
$$




\section{Appendix B}

\section{Calculation of mixing coefficients and lifetimes of the $\mathrm{CO}$ $\mathrm{e}^{3} \Sigma^{-}(v=12)$ levels interacting with $\mathrm{A}^{1} \Pi(v=8)$}

The effective Hamiltonian described in the previous section describes the electronic structure of diatomic molecules within one vibrational and electronic state. This description is not a good approximation when rotational levels of different electronic states are near degenerate.

In the $\mathrm{CO}$ molecule, the $\mathrm{A}^{1} \Pi$ state (which has a large transition strength from/to the electronic ground state $\mathrm{X}^{1} \Sigma^{+}$) is embedded in a triplet manifold (see Fig. B.1). Any local perturbation follows the selection rule $\Delta J=0$. The degree of mixing due to perturbations is large, when interacting rotational levels are close in energy. Figure B.2 shows a perturbation diagram of $\mathrm{CO}$ from the $\mathrm{PhD}$ thesis of $\mathrm{A}$. Lefloch. The figure shows the energy of the different electronic states in the CO molecule as function of the rotational state $J$. I marked the $\mathrm{A}^{1} \Pi(v=8)$ and $\mathrm{e}^{3} \Sigma^{-}(v=12)$ pair of interacting states with red and blue colored lines, respectively. This pair of states is near degenerate at low $J$ (only low $J$ levels are accessible with high beam density by optical pumping of the rotationally cold molecular beam), which is important to obtain strong mixing. For comparison, the figure also shows the $\mathrm{A}^{1} \Pi(v=1) \sim \mathrm{d}^{3} \Delta_{1}\left(v^{\prime}=5\right)$ interaction pair exploited by Blokland et al. (green and brown lines) (185). 
B. Calculation of mixing coefficients and lifetimes of the $\mathrm{CO} \mathrm{e}^{3} \Sigma^{-}(v=12)$ levels interacting with $\mathrm{A}^{1} \Pi(v=8)$

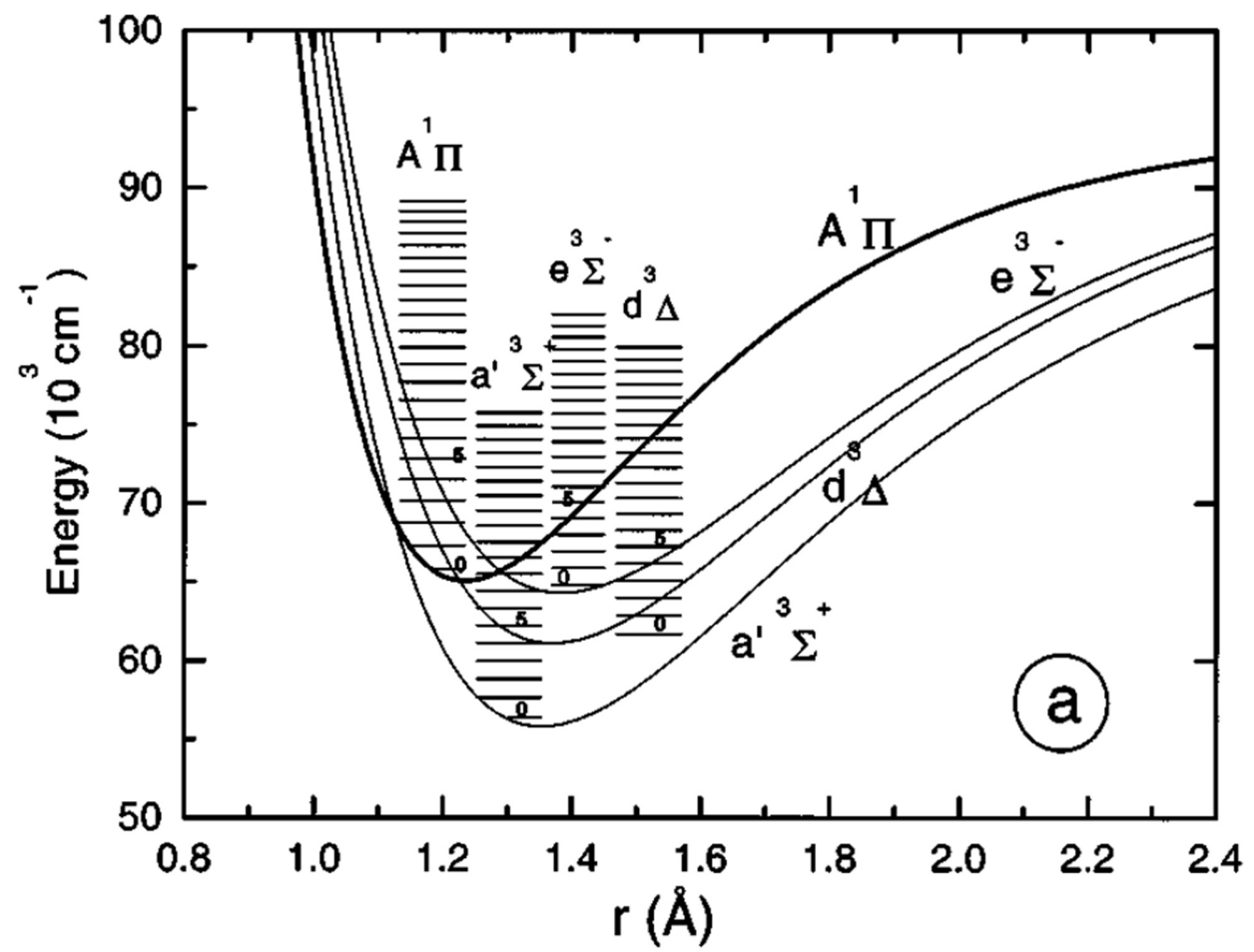

Figure B.1: Triplet manifold in the CO molecule - The $\mathrm{A}^{1} \Pi$ state of CO is embedded in a triplet manifold, giving rise to perturbations between different electronic states. Reprinted with permission from (179). Copyright 2000, AIP Publishing LLC.

I now explain the quantitative treatment between rotational levels of $\mathrm{A}^{1} \Pi(v=$ 8) and $\mathrm{e}^{3} \Sigma^{-}(v=12)$ of same parity and $J$ interacting via off diagonal spin-orbit interaction.

The $\mathrm{e}^{3} \Sigma^{-}$state has a total electron spin of $S=1$, a molecule fixed projection of the total electronic angular momentum on the $z$-axis of $\Lambda=0$. The projection of $\mathbf{S}$ on the $z$-axis can take the values $\Sigma=+1,0,-1$. As $\Lambda=0, \Omega=\Lambda+\Sigma$ takes the same values as $\Sigma$. We use basis set of linear combinations of Hund's case a) basis functions (written in the abbreviated form $|S \Lambda \Sigma\rangle$ ), chosen to be symmetrized with respect to a reflection 
in a plane containing the internuclear axis.

$$
\begin{aligned}
& \Phi_{1}\left(\mathrm{e}^{3} \Sigma^{-}\right)=\frac{1}{\sqrt{2}}\left(\left|1,0^{-}, 1\right\rangle+\left|1,0^{-},-1\right\rangle\right) \quad((\mathrm{e}) \text {-symmetry }) \\
& \Phi_{2}\left(\mathrm{e}^{3} \Sigma^{-}\right)=\left|1,0^{-}, 0\right\rangle \quad((\mathrm{e}) \text {-symmetry }) \\
& \Phi_{3}\left(\mathrm{e}^{3} \Sigma^{-}\right)=\frac{1}{\sqrt{2}}\left(\left|1,0^{-},-1\right\rangle+\left|1,0^{-}, 1\right\rangle\right) \quad((\mathrm{f}) \text {-symmetry })
\end{aligned}
$$

The states $\Phi_{2}\left(\mathrm{e}^{3} \Sigma^{-}\right)={ }^{3} \Sigma_{0}^{-}(\mathrm{e})$ and $\Phi_{1}\left(\mathrm{e}^{3} \Sigma^{-}={ }^{3} \Sigma_{1}^{-}(\mathrm{e})\right.$ interact via the $\mathbf{S}$-uncoupling operator (Equation A.39)

$$
\begin{aligned}
& \left\langle\Lambda, S, \Sigma=0, J, \Omega=0\left|-B_{v} J^{ \pm} S^{\mp}\right| \Lambda, S, \Sigma \pm 1, J, \Omega \pm 1\right\rangle \\
= & -B_{v} \sqrt{S(S+1) \cdot J(J+1)}=-B_{\mathrm{e}} \sqrt{2 \cdot J(J+1)},
\end{aligned}
$$

where $B_{\mathrm{e}}$ is the rotational constant of the $\mathrm{e}^{3} \Sigma^{-}$state, which is - together with other relevant molecular constants - given in Table B.1.

Table B.1: Molecular constants of the Hamiltonian matrix.

\begin{tabular}{cccc}
\hline \hline & Parameter $\left.^{\mathrm{a}}\right)$ & Value $\left.^{\mathrm{a}}\right) / \mathrm{cm}^{-1}$ & Physical origin \\
\hline $\mathrm{e}^{3} \Sigma^{-}(v=12)$ & $E_{\mathrm{e}}$ & 75583.112 & Vibronic energy \\
& $B_{\mathrm{e}}$ & 1.07159 & Rotational constant \\
$\mathrm{A}^{1} \Pi(v=8)$ & $\lambda$ & $0.783^{\mathrm{b}}$ & Spin-Spin constant \\
& $E_{\mathrm{A}}$ & 75632.97 & Vibronic energy \\
Off diagonal & $B_{\mathrm{A}}$ & 1.41567 & Rotational constant \\
\hline \hline
\end{tabular}

a) From Ref. (183).

b) Calculated from $C=-0.522 \mathrm{~cm}^{-1}$ via $C=-\frac{2}{3} \lambda$.

In addition, we include the spin-spin interaction with the matrix elements as given by Equation A.41. The operators $-B\left(L^{+} S^{-}+L^{-} S^{+}\right)$and $J^{+} L^{-}+J^{-} L^{+}$do not contribute, because the $\mathrm{e}^{3} \Sigma^{-}(v=12)$ state has zero electronic orbital angular momentum. The spin-rotation operator $\hat{H}_{\mathrm{SR}}$ does in principle contribute, but the contribution is small and thus neglected in the calculation.

The $\mathrm{A}^{1} \Pi(v=8)$ state is also described with (e)/(f)-symmetrized basis functions of the 
B. Calculation of mixing coefficients and lifetimes of the $\mathrm{CO} \mathrm{e}^{3} \Sigma^{-}(v=12)$ levels interacting with $\mathrm{A}^{1} \Pi(v=8)$

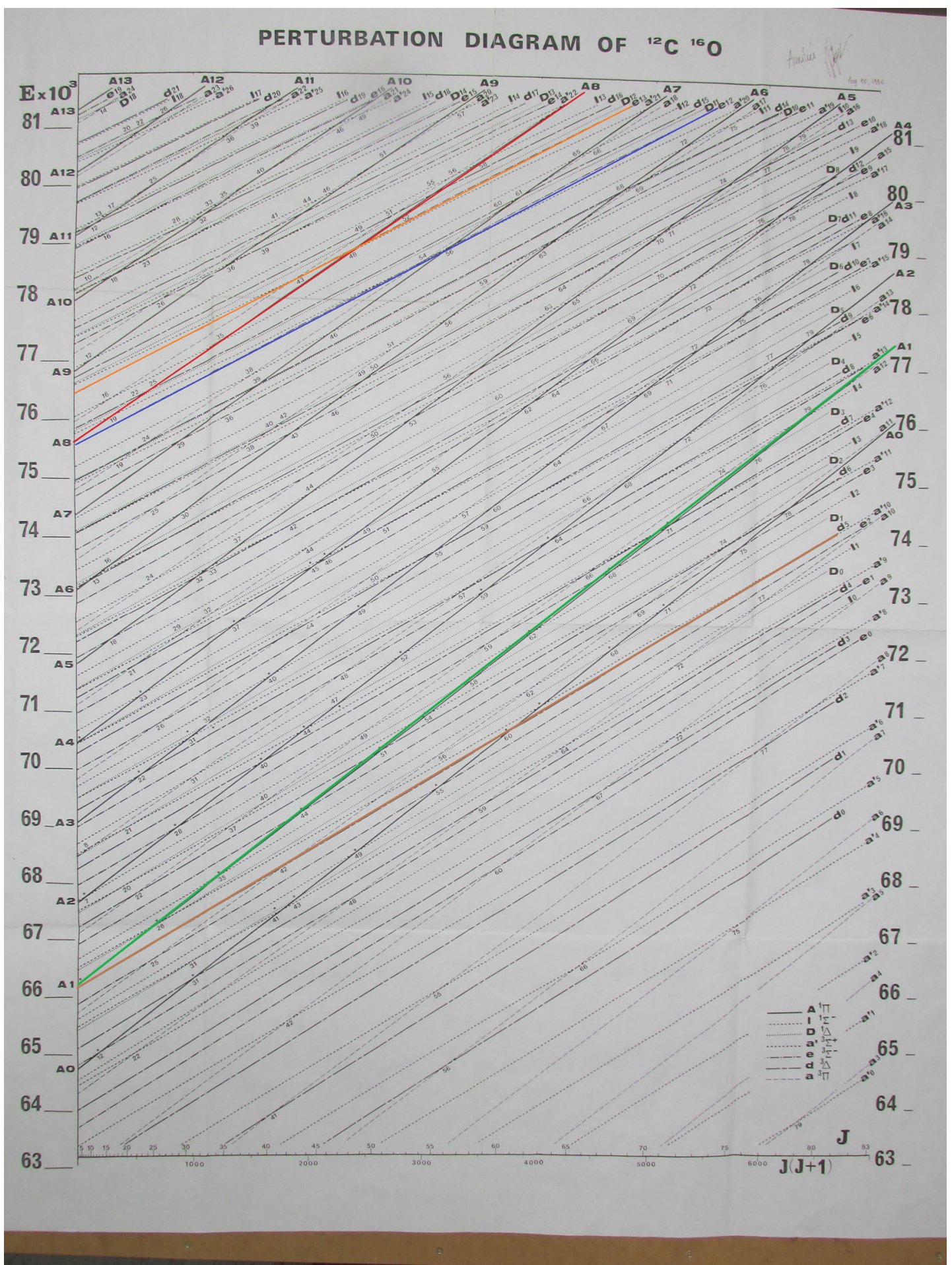

Figure B.2: Perturbation diagram of CO - The interaction pair $\mathrm{e}^{3} \Sigma^{-}(v=12) \sim$ $\mathrm{A}^{1} \Pi(v=8)$ is marked with read and blue lines, respectively. For comparison, the $\mathrm{A}^{1} \Pi(v=$ 1) $\sim \mathrm{d}^{3} \Delta_{1}\left(v^{\prime}=5\right)$ interaction pair exploited by Blokland et al. (green and brown lines) is also given. Figure adapted from Ref. (188). 
abbreviated form $|S \Lambda \Sigma\rangle$ given by

$$
\begin{array}{ll}
\Phi_{4}\left(\mathrm{~A}^{1} \Pi\right)=\frac{1}{\sqrt{2}}(|0,1,0\rangle+|0,-1,0\rangle) & ((\mathrm{e}) \text {-symmetry }) \\
\Phi_{5}\left(\mathrm{~A}^{1} \Pi\right)=\frac{1}{\sqrt{2}}(|0,1,0\rangle-|0,-1,0\rangle) & ((\mathrm{f}) \text {-symmetry }) .
\end{array}
$$

The basis states between $\mathrm{A}^{1} \Pi$ and $\mathrm{e}^{3} \Sigma^{-}$interact via the off diagonal spin orbit matrix element

$$
A_{10}^{S} \equiv\left\langle{ }^{1} \Pi((\mathrm{e}) /(\mathrm{f}))\left|H_{\mathrm{SO}}\right|^{3} \Sigma_{1}^{-}((\mathrm{e}) /(\mathrm{f}))\right\rangle,
$$

following the selection rules $\Delta \Lambda=-\Delta \Sigma= \pm 1$ and (e) $\leftrightarrow$ (f).

The evaluation of the matrix elements in the basis of $\left\{\Phi_{1}\left(\mathrm{e}^{3} \Sigma^{-}\right), \Phi_{2}\left(\mathrm{e}^{3} \Sigma^{-}\right), \Phi_{4}\left(\mathrm{~A}^{1} \Pi\right)\right\}$ [(e)-symmetry] and $\left\{\Phi_{3}\left(\mathrm{e}^{3} \Sigma^{-}\right), \Phi_{5}\left(\mathrm{~A}^{1} \Pi\right)\right\}[(\mathrm{f})$-symmetry] results in the following Hamiltonian matrix:

which has the elements

$$
\left(\begin{array}{ccccc}
H_{1} & H_{2} & A_{10}^{S} & 0 & 0 \\
H_{2} & H_{3} & 0 & 0 & 0 \\
A_{10}^{S} & 0 & H_{4} & 0 & 0 \\
0 & 0 & 0 & H_{5} & A_{10}^{S} \\
0 & 0 & 0 & A_{10}^{S} & H_{6}
\end{array}\right)
$$

$$
\begin{aligned}
& H_{1}=E_{\mathrm{e}}+B_{\mathrm{e}} J(J+1)+\frac{2}{3} \lambda \\
& H_{2}=-\sqrt{2} B_{\mathrm{e}}[2 J(J+1)]^{0.5} \\
& H_{3}=E_{\mathrm{e}}+B_{\mathrm{e}}[J(J+1)+2]-\frac{4}{3} \lambda \\
& H_{4}=E_{\mathrm{A}}+B_{\mathrm{A}}[J(J+1)-1] \\
& H_{5}=E_{\mathrm{e}}+B_{\mathrm{e}} J(J+1)+\frac{2}{3} \lambda \\
& H_{6}=E_{\mathrm{A}}+B_{\mathrm{A}}[J(J+1)-1] .
\end{aligned}
$$

The Hamiltonian matrix factors into a $3 \times 3$ and a $2 \times 2$ parity block, which is due to the selection rule (e) $\leftrightarrow$ (f) for molecular interactions.

Calculating the eigenvalues and eigenvectors of the Hamiltonian matrix yields the energies as well as the mixing coefficients $\alpha_{i}^{2}, \beta_{i}^{2}, \gamma_{i}^{2}, \delta_{i}^{2}$ and $\epsilon_{i}^{2}$, which describe the wave functions defined by

$$
\psi_{i}=\alpha_{i} \cdot \Phi_{1}\left(\mathrm{e}^{3} \Sigma^{-}\right)+\beta_{i} \cdot \Phi_{2}\left(\mathrm{e}^{3} \Sigma^{-}\right)+\gamma_{i} \cdot \Phi_{3}\left(\mathrm{e}^{3} \Sigma^{-}\right)+\delta_{i} \cdot \Phi_{4}\left(\mathrm{~A}^{1} \Pi\right)+\epsilon_{i} \cdot \Phi_{5}\left(\mathrm{~A}^{1} \Pi\right) .
$$



levels interacting with $\mathrm{A}^{1} \Pi(v=8)$

The mixing coefficients for the different levels in $J=1$ and $J=2$ of $\mathrm{e}^{3} \Sigma^{-}(v=12)$ are given in Table B.2. For each $J$, states are labeled by $\mathrm{F}_{3}, \mathrm{~F}_{2}$ and $\mathrm{F}_{1}$ starting from the highest to the lowest energy, corresponding to $J=N-1, N, N+1$. At $J=0$, the energy difference between the (deperturbed) $\mathrm{e}^{3} \Sigma^{-}(v=12)$ and the $\mathrm{A}^{1} \Pi(v=8)$ states is large (approx. $50 \mathrm{~cm}^{-1}$ ) compared to the off diagonal spin-orbit interaction of $A_{10}^{S}=-4.03 \mathrm{~cm}^{-1}$. Thus, the predominantly $\mathrm{e}^{3} \Sigma^{-}(v=12)$ levels have relatively small $\mathrm{A}^{1} \Pi$ character (given by $\delta_{i}^{2}$ and $\epsilon_{i}^{2}$ in Table B.2.

Table B.2: Energy, lifetimes, and mixing coefficients for the predominantly $\mathrm{e}^{3} \Sigma^{-}(v=12)$ state interacting with $\mathrm{A}^{1} \Pi(v=8)$.

\begin{tabular}{|c|c|c|c|c|c|c|c|c|c|c|}
\hline \multirow{2}{*}{\multicolumn{2}{|c|}{ Label }} & \multicolumn{7}{|c|}{ Calculation } & \multicolumn{2}{|c|}{ Experimental } \\
\hline & & $\mathrm{E} / \mathrm{cm}^{-1}$ & $\alpha^{2}$ & $\beta^{2}$ & $\gamma^{2}$ & $\delta^{2}$ & $\epsilon^{2}$ & $\tau / \mu \mathrm{s}$ & $\mathrm{E} / \mathrm{cm}^{-1}$ & $\tau / \mu \mathrm{s}$ \\
\hline \multirow[t]{3}{*}{$J=1$} & $\mathrm{~F}_{3}$ & 75589.0 & 0.4224 & 0.5743 & 0.0000 & 0.0033 & 0.0000 & 1.88 & 75588.9 & 1.8 \\
\hline & $\mathrm{F}_{2}$ & 75585.5 & 0.0000 & 0.0000 & 0.9933 & 0.0000 & 0.0067 & 1.15 & & \\
\hline & $\mathrm{F}_{1}$ & 75582.9 & 0.5708 & 0.4257 & 0.0000 & 0.0035 & 0.0000 & 1.82 & 75582.7 & 1.5 \\
\hline \multirow[t]{3}{*}{$J=2$} & $\mathrm{~F}_{3}$ & 75595.5 & 0.4537 & 0.5426 & 0.0000 & 0.0037 & 0.0000 & 1.76 & 75595.0 & 1.7 \\
\hline & $\mathrm{F}_{2}$ & 75589.8 & 0.0000 & 0.0000 & 0.9936 & 0.0000 & 0.0064 & 1.20 & & \\
\hline & $\mathrm{F}_{1}$ & 75585.0 & 0.5398 & 0.4573 & 0.0000 & 0.0029 & 0.0000 & 2.05 & 75584.4 & 1.8 \\
\hline
\end{tabular}

From the mixing coefficients and the unperturbed lifetimes (10 ns for the $\mathrm{A}^{1} \Pi$ state and $5 \mu$ s for the $\mathrm{e}^{3} \Sigma^{-}$states) the lifetimes of the mixed states $\tau$ can be calculated via the equation

$$
\frac{1}{\tau}=\frac{1-\left(\delta^{2}+\epsilon^{2}\right)}{5 \mu \mathrm{s}}+\frac{\delta^{2}+\epsilon^{2}}{10 \mathrm{~ns}} .
$$

These calculated lifetimes are also included in Table B.2. The lifetimes of the $\mathrm{F}_{1}$ and $\mathrm{F}_{3}$ levels are very similar and agree with the experimentally measured lifetimes within the experimental error. The lifetimes of the $\mathrm{F}_{2}$ levels are significantly shorter as the partial $\mathrm{A}^{1} \Pi$ character of the wave functions is larger. However, the $\mathrm{F}_{2}$ levels cannot be accessed by optical pumping from $\mathrm{a}^{3} \Pi_{1}$, due to the $\Delta \Sigma=0$ spin selection rule. 


\section{Appendix C}

\section{Time delays in a pump-dump-sweep-probe molecular beam scattering experiment}

Fig. C.1 shows the time delays of a typical pump-dump-sweep-porbe molecular beam scattering experiment as described in Section 6.2. The exact delays between firing the different lasers depend on the speed of the molecular beam and the values given in the figure belong to a mixture of $60 \%$ NO seeeded in Argon as a carrier gas.

The time delays are controlled using three delay/pulse generators (model DG535, Stanford Research Systems, Inc.). The molecular beam is generated by the supersonic jet expansion between $t=0$ and $170 \mu \mathrm{s}$. Then the vibrational excitation with the pump, dump and sweep lasers is performed in the differential chamber (compare Fig. 3.1 on page 40) at $445 \mu \mathrm{s}$ (optimized to the strongest laser induced fluorescence signal). The incoming beam is probed in front of the surface in the UHV chamber at $707 \mu \mathrm{s}$ (without electric field). The scattered beam has to be detected at a later time $(726.4 \mu \mathrm{s})$. In case an electric field is used for orienting molecules prior to the collision, the electric field is pulsed to ground shortly before the REMPI detection. This pulsing has to occur after the NO molecules collided with the surface (because otherwise the orientation is lost) and before the REMPI detection (otherwise the generated ions are not guided to the microchannel plate (MCP) detector and line positions shift due to the Stark effect). 
C. Time delays in a pump-dump-sweep-probe molecular beam scattering experiment

Another detail is that the voltage of the MCP is also pulsed (not shown in Fig. C.1). The voltage on the front plate was typically pulsed up from $1500 \mathrm{~V}$ to $1900 \mathrm{~V} 2 \mu \mathrm{s}$ before firing the REMPI laser. Pulsing the MCP voltage leads to a better ion detection efficiency, because background ions lead to a saturation of the electron multiplier in the MCP.

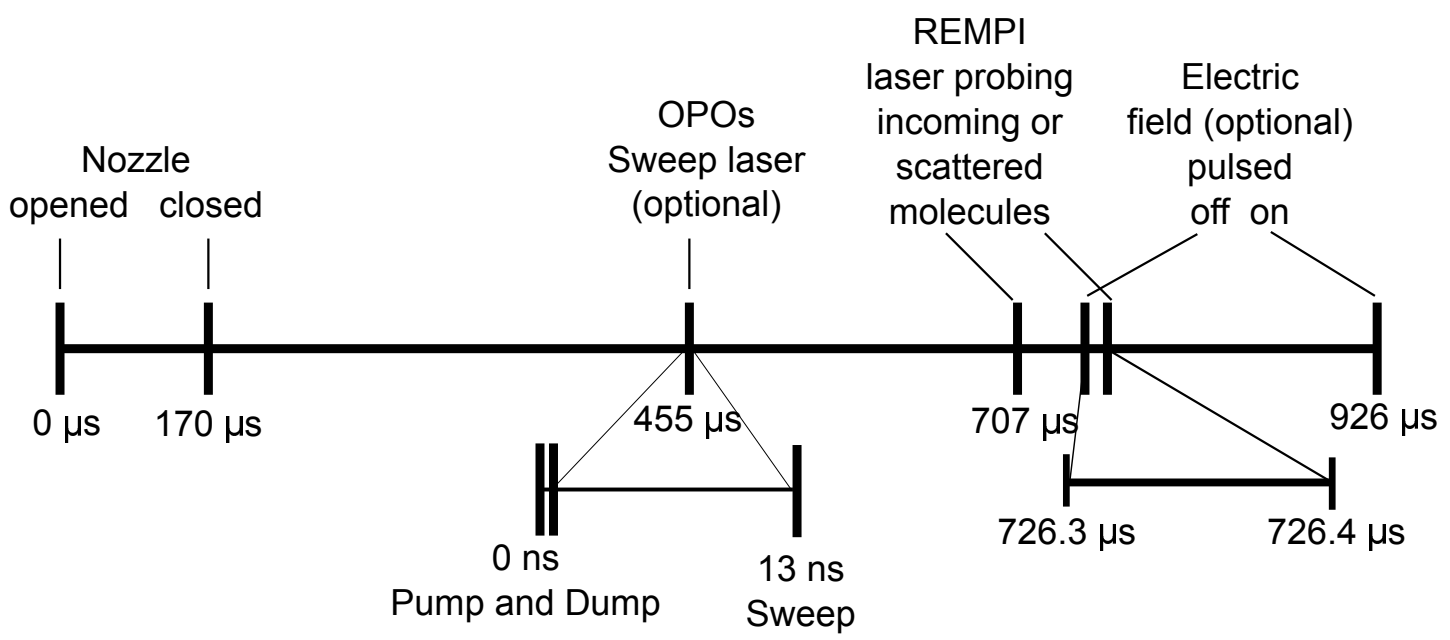

Figure C.1: Time delays in pump-dump-sweep-probe molecular beam scattering experiment. - The sequence of nozzle opening, firing of the pump-dump and sweep lasers and the REMPI detection (either of the incidence or the scattered molecular beam) is shown for a gas mixture of $60 \%$ NO seeeded in Argon. In case the molecules are oriented prior to the collision an electric field is used, which is pulsed to ground prior to REMPI detection. Figure adapted from the masters thesis of B. C. Krüger (140) with his permission. 


\section{Appendix D}

\section{Mathematica notebook for calculating line positions in the $\gamma$-bands of NO}

A major step for the data analysis of the REMPI spectra when scattering NO from $\mathrm{Au}(111)$ is to calculate accurate line positions of all rotational branches in the $\gamma$-bands of NO. Very accurate line positions are provided as tables in the Lifbase spectra simulation software (189). Unfortunately, these are only given up to a vibrational state of $v^{\prime}=5$ in the $\mathrm{A}^{2} \Sigma^{+}$state. I wrote a Wolfram Mathematica notebook to reproduce the line positions from spectroscopic constants from the literature.

The notebook uses Brown's Hamiltonian as described by Amiot et al. (144) to describe the electronic ground state $\mathrm{X}^{2} \Pi$ with molecular constants from Ref. (190). The constants for the $\Lambda$-splitting are taken from Danielak et al. (104) and the hyperfine splitting is neglected. The same reference (104) also provided molecular constants for the $\mathrm{A}^{2} \Sigma^{+}$state. 
D. Mathematica notebook for calculating line positions in the $\gamma$-bands of NO

\section{Calculation of line positions using Brown's Hamiltonian}

\section{Ground State X}

Molecular constants

Molecular constants are taken from Amiot JMS v94 150-172 (1982), except for the lambda splitting constants p and q from J Mol. Spec. 181, p 394 (1997) : "Danielak et al, Reinvestigation of the Emission g Band System (A2S/-X 2P) of the NO Molecule"

$\ln [1]:=$ (*vibrational energy*)

Gvalues $=\{0 ., 1875.989,3723.887584,5543.744427,7335.602566,9099.496772$, $10835.4498,12543.47257,14223.56027,15875.68886,17499.81434,19095.86584$, $20663.74636,22203.32257,23714.42691,25196.84627,26650.3196$, $28074.53079,29469.10186,30833.58502,32167.45539,33470.10789$ \};

$[2]=(*$ Spin orbit splitting *)

Avalues $=\{123.13361,122.889,122.634851,122.369369,122.09145$,

$121.80029,121.49416,121.17045,120.82828,120.46445,120.07645$,

$119.66327,119.21941,118.74181,118.22581,117.6677,117.0596$,

$116.39869,115.67594,114.88285,114.01360,113.05406,112.00095$ \} ;

Advalues $=\{17.217,16.384,15.443,14.493,13.468,12.38,11.136$,

$10.004,8.577,7.272,5.801,3.854,2.032,-0.214,-2.481,-5.09$,

$-8.17,-11.37,-15.11,-19.51,-24.09,-28.72,-34.86\} * 10 \wedge(-5)$;

$\ln [4]:=(*$ Rotation $*)$

Bvalues $=\{1.69611363,1.67854221,1.66094026,1.64330405,1.62563277$

$1.60791947,1.59016139,1.57235322,1.55448322,1.5365486,1.51853385$,

$1.50042899,1.48221531,1.46388212,1.44540152,1.4267538,1.4079099$,

$1.3888362,1.3694984,1.34985,1.3298521,1.3094217,1.2885226\}$;

Ddvalues $=\{5.47641,5.49197,5.50917,5.52657,5.54595,5.56581,5.58701$,

$5.61131,5.63497,5.6664,5.69483,5.72941,5.76618,5.81139,5.86022$ ，

$5.91587,5.981,6.05393,6.1381,6.2333,6.3498,6.4655,6.5952\} * 10^{\wedge}(-6)$;

Hvalues $=\{1.39,1.11,0.89,0.65,0.38,0.04,-0.35,-0.79,-1.28$,

$-1.82,-2.42,-3.1,-3.91,-4.89,-6.09,-7.57,-9.41$,

$-11.66,-14.4,-17.5,-21.2,-25.3,-29.7\} * 10^{\wedge}(-12)$;

:= (*hyperfine splitting*)

pe $=1.1681 * 10^{\wedge}(-2)$.

$\alpha$ pe $=5.9 * 10^{\wedge}(-5)$;

$\mathrm{qe}=1.031 * 10^{\wedge}(-4)$

$\alpha q e=-5.2 * 10^{\wedge}(-7)$ 
2 LinePositionsForThesis3.nb

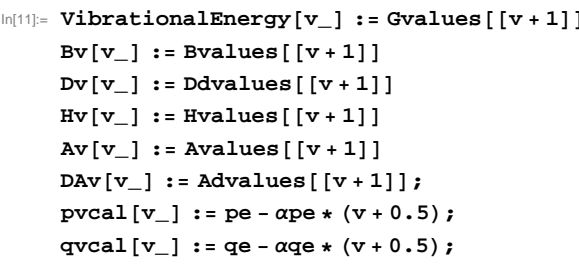

Calculation of the energy levels

First we calculate the energy levels with spin orbit splitting neglecting hyperfine splitting

The Hamiltonian has been evaluated in the publication: "Amiot et al., Can. J. Phys., Vol 56, page 251, 1978". (Browns Hamiltonian)

TABLE II

Hamiltonian Matrix Elements for $\mathrm{OD} X^{2} \Pi$ State in a Parity Case (a) Basis Set: Effective Hamiltonian of Brown (4l)

\begin{tabular}{|c|c|c|c|c|c|}
\hline$T$ & $\begin{array}{l}1,1 \\
2,2\end{array}$ & $\begin{array}{l}1 \\
1\end{array}$ & $P$ & 2,2 & $\mp 0.5(\mathrm{~J}+0.5)$ \\
\hline A & $\begin{array}{l}1,1 \\
2,2\end{array}$ & $\begin{array}{r}0.5 \\
-0.5\end{array}$ & $P_{0}$ & $\begin{array}{l}2,2 \\
1,2\end{array}$ & $\begin{array}{l}\mp 0.5(\mathrm{~J}+0.5)(\mathrm{z}+2) \\
\pm 0.25 \mathrm{z}^{0.5}(\mathrm{~J}+0.5)\end{array}$ \\
\hline$A_{D}$ & $\begin{array}{l}1,1 \\
2,2\end{array}$ & $\begin{array}{l}0.5 z \\
-0.5(z+2)\end{array}$ & $P_{H}$ & $\begin{array}{l}2,2 \\
1,2\end{array}$ & $\begin{array}{l}\mp 0.5(z+4)(\mathrm{J}+0.5)^{3} \\
\pm 0.5 z^{0.5}(\mathrm{~J}+0.5)^{3}\end{array}$ \\
\hline$A_{H}$ & $\begin{array}{l}1,1 \\
2,2 \\
1,2\end{array}$ & $\begin{array}{l}z^{2} \\
-(z+2)^{2} \\
z=.5\end{array}$ & $q$ & $\begin{array}{l}2,2 \\
1,2\end{array}$ & $\begin{array}{l}\mp(\mathrm{J}+0.5) \\
\pm 0.5 \mathrm{z}^{0.5}(\mathrm{~J}+0.5)\end{array}$ \\
\hline B & $\begin{array}{l}1,1 \\
2,2 \\
1,2\end{array}$ & $\begin{array}{l}z \\
z+2 \\
-z^{0.5}\end{array}$ & $q_{0}$ & $\begin{array}{l}1,1 \\
2,2 \\
1,2\end{array}$ & $\begin{array}{l}\mp 0.5 z(\mathrm{~J}+0.5) \\
\mp 0.5(32+4)(\mathrm{J}+0.5) \\
\left.+0.5 z^{0.5}(\mathrm{z}+2)(\mathrm{l})+0.5\right)\end{array}$ \\
\hline$D$ & $\begin{array}{l}1,1 \\
2,2 \\
1,2\end{array}$ & $\begin{array}{l}-z(z+1) \\
-(z+1)(z+4) \\
2 z^{0.5}(z+1)\end{array}$ & $a_{H}$ & $\begin{array}{l}1,1 \\
2,2 \\
1,2\end{array}$ & $\begin{array}{l}\mp z(\mathrm{~J}+0.5)^{3} \\
\mp 2(\mathrm{z}+2)(\mathrm{J}+0.5)^{3} \\
\pm 0.5 z^{0.5}(z+4)(\mathrm{J}+0.5)^{3}\end{array}$ \\
\hline H & $\begin{array}{l}1,1 \\
2,2 \\
1,2\end{array}$ & $\begin{array}{r}z(z+1)(z+2) \\
\quad(z+1)\left(z^{2}+8 z+8\right) \\
-z^{0.5}(z+1)(3 z+4)\end{array}$ & $\gamma$ & $\begin{array}{l}2,2 \\
1,2\end{array}$ & ${ }^{-1} 0.5 z^{0.5}$ \\
\hline ᄂ & $\begin{array}{l}1,1 \\
2,2\end{array}$ & $\begin{array}{ll}-z(z+1)^{2} & (z+4) \\
-(z+1)^{2} & \left(z^{2}+12 z+16\right)\end{array}$ & $\gamma_{D}$ & $\begin{array}{l}1,1 \\
2,2 \\
1,2\end{array}$ & $\begin{array}{l}-0.5 z \\
-0.5(3 z+4) \\
0.5 z^{0.5}(z+2)\end{array}$ \\
\hline & 1,2 & $4 z^{0.5}(z+1)^{2}(z+2)$ & $\gamma_{\mathrm{H}}$ & $\begin{array}{l}1,1 \\
2,2 \\
1,2\end{array}$ & $\begin{array}{l}-z-z^{2} \\
-2(z+1)(z+2) \\
0.5 z^{0.5}\left(z^{2}+5 z+4\right)\end{array}$ \\
\hline
\end{tabular}

Label 1 refers to ${ }^{2} \Pi \frac{3}{2}$, label 2 to ${ }^{2} \Pi \frac{1}{2}, z=(J+0.5)^{2}-1=(J-0.5)(J+1.5)$. When two signs are quoted the lower is for $f$ levels and the upper for $e$ levels. 
D. Mathematica notebook for calculating line positions in the $\gamma$-bands of NO

Definition of the e - and f-parity blocks :

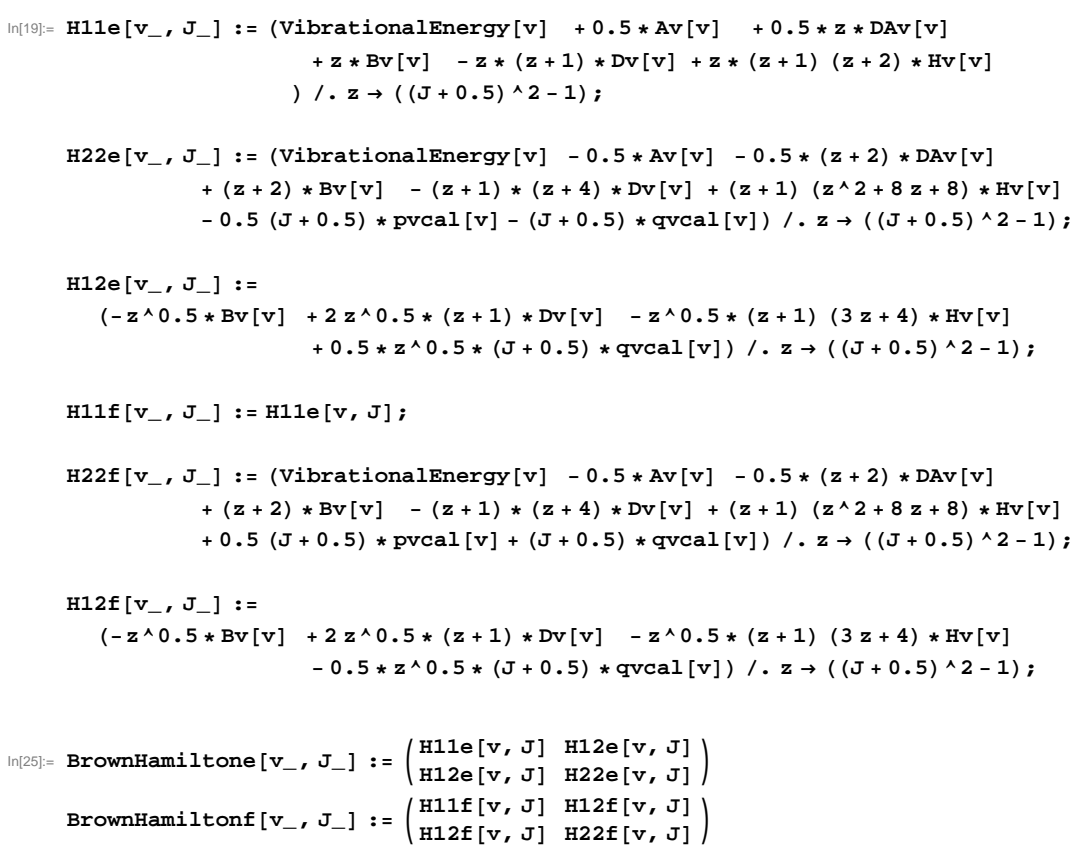


4 | LinePositionsForThesis3.nb

Generation of energy table

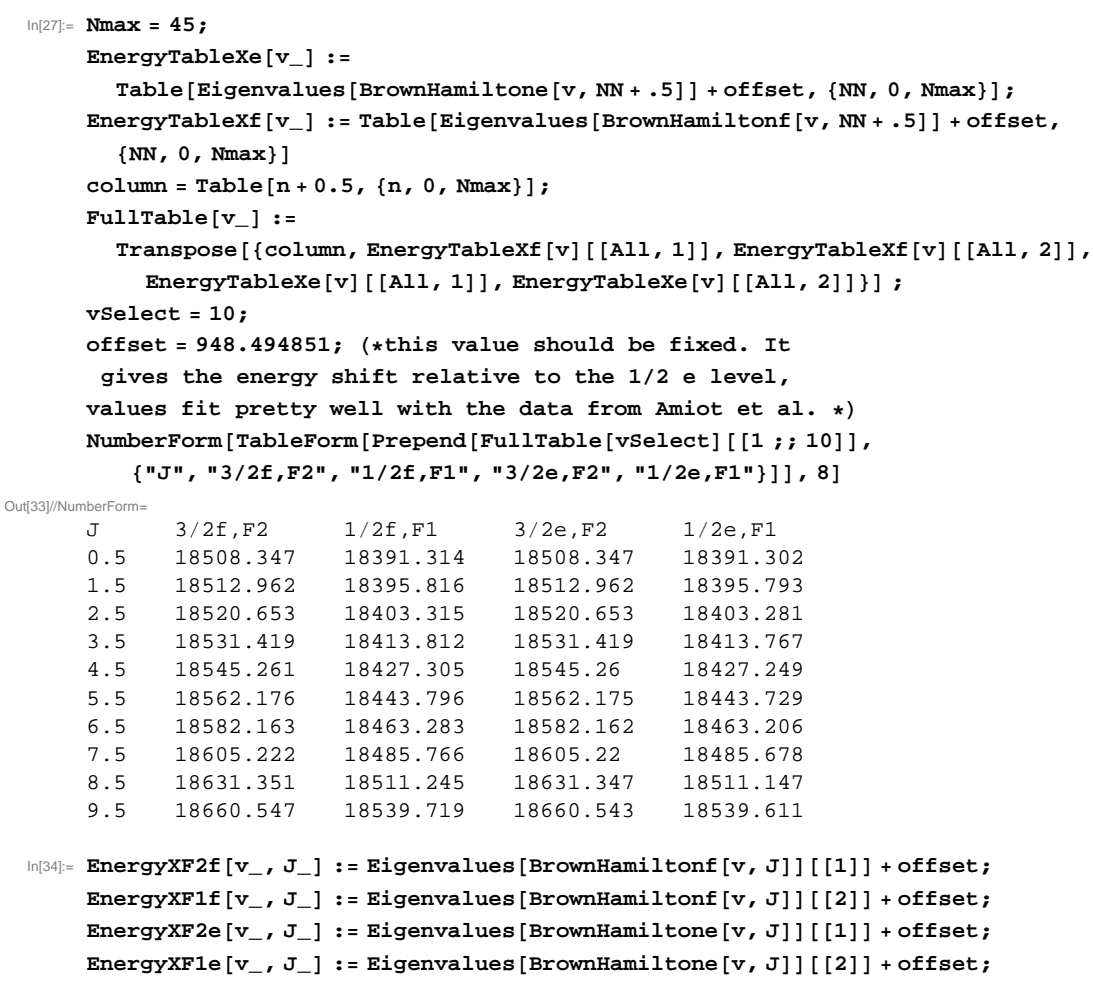

\section{Excited A state}

Molecular constants from J Mol. Spec. 181, p 394 (1997) : "Danielak et al, Reinvestigation of the Emission g Band System (A2S/-X 2P) of the NO Molecule" In the article the value for gamma was contrained to certain values of up to $A(v=4)$ only. The agreement of linepositions is much better without this parameter anyhow. 
D. Mathematica notebook for calculating line positions in the $\gamma$-bands of NO

LinePositionsForThesis3.nb | $\mathbf{5}$

Molecular constants

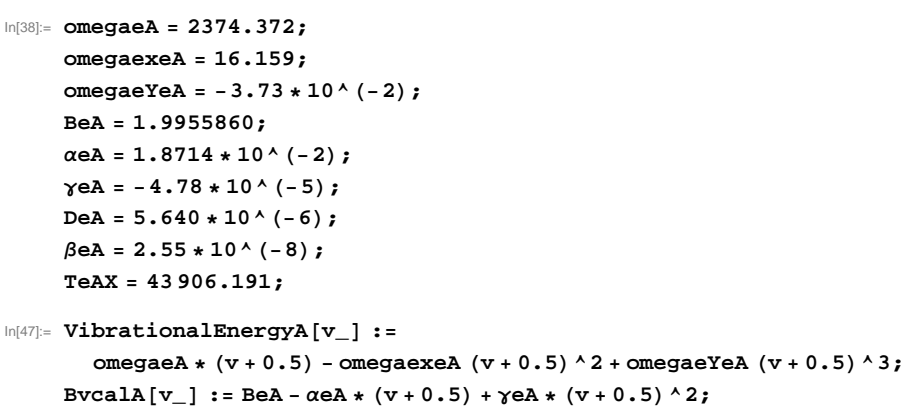

Calculation of energy tables

\section{TABLE III}

Hamiltonian Matrix Elements for $\mathrm{OD} A^{2} \Sigma^{+}$State in a Parity Case (a) Basis Set

\begin{tabular}{|c|c|c|c|}
\hline \multirow{2}{*}{$T$} & 1 & \multirow{2}{*}{ L } & $-(\mathrm{J}-0.5)^{4} \quad(\mathrm{~J}+0.5)^{4}$ \\
\hline & 1 & & $-(J+0.5)^{4} \quad(J+1.5)^{4}$ \\
\hline \multirow{2}{*}{ B } & $(\mathrm{J}-0.5) \quad(\mathrm{J}+0.5)$ & \multirow{2}{*}{$\gamma$} & $0.5(\mathrm{~J}-0.5)$ \\
\hline & $(\mathrm{J}+0.5) \quad(\mathrm{J}+1.5)$ & & $0.5(\mathrm{~J}+1.5)$ \\
\hline \multirow{2}{*}{ D } & $-(\mathrm{J}-0.5)^{2}(\mathrm{~J}+0.5)^{2}$ & \multirow{2}{*}{$\gamma_{D}$} & \multirow{2}{*}{$\begin{array}{l}0.5(\mathrm{~J}-0.5) \mathrm{J}(\mathrm{J}+1) \\
0.5(\mathrm{~J}+1.5) \mathrm{J}(\mathrm{J}+1)\end{array}$} \\
\hline & $-(\mathrm{J}+0.5)^{2}(\mathrm{~J}+1.5)^{2}$ & & \\
\hline \multirow[b]{2}{*}{ H } & $(\mathrm{J}-0.5)^{3}(\mathrm{~J}+0.5)^{3}$ & \multirow[b]{2}{*}{$\gamma_{H}$} & $0.5(\mathrm{~J}-0.5) \mathrm{J}^{2}(\mathrm{~J}+1)^{2}$ \\
\hline & $(\mathrm{J}+0.5)^{3}(\mathrm{~J}+1.5)^{2}$ & & $0.5(\mathrm{~J}+1.5) \mathrm{J}^{2}(\mathrm{~J}+1)^{2}$ \\
\hline
\end{tabular}

Upper line refers to $e$ levels and lower line to $f$ levels.

$[50]=$ EnergyAe $\left[\mathbf{v}_{-}, J_{-}\right]:=$TeAX + VibrationalEnergy $[\mathbf{v}]+$

BvcalA $[v] *(J-0.5)(J+0.5)-\operatorname{DvcalA}[v](J-0.5) \wedge 2 *(J+0.5) \wedge 2$

EnergyAf $\left[v_{-}, J_{-}\right]:=$TeAX + VibrationalEnergyA $[v]+$

BvcalA $[\mathrm{v}] *(\mathrm{~J}+0.5)(\mathrm{J}+1.5)-\operatorname{DvcalA}[\mathrm{v}](\mathrm{J}+0.5) \wedge 2 *(\mathrm{~J}+1.5) \wedge 2$ 
6 | LinePositionsForThesis3.nb

Generation of energy table

$\ln [52]:=\operatorname{Nmax} \mathbf{A}=50$;

EnergyVektorAe $\left[\mathrm{v}_{-}\right]:=\operatorname{Table}[$ EnergyAe $[\mathrm{v}, \mathrm{NN}+.5]+\operatorname{offset} A,\{\mathrm{NN}, 0, \operatorname{NmaxA}\}]$

EnergyVektorAf $\left[v_{-}\right]:=\operatorname{Table}\left[E n e r g y A f\left[v_{1} N N+.5\right]+\operatorname{offset} A,\{N N, 0, \operatorname{NmaxA}\}\right]$

columnA $=$ Table $[n+0.5,\{n, 0, N \operatorname{maxA}\}]$;

FullTableA [v_] : =

Transpose [ $\{$ columnA, EnergyVektorAf [v], EnergyVektorAe [v] \}] ;

vSelectA $=2$;

offset $A=0$;

NumberForm [

TableForm [Prepend [FullTableA [vSelectA] [ $[1 ; ; 10]],\{$ J", "f,F2", "e,F1" $\}]$ ], 7]

(* In the table, the first row is messed up, there is no omega $3 / 2$,

$\mathrm{J}=0.5$ levels and this messes up the order*)

$\begin{array}{lll}\begin{array}{c}\text { Out[59//NumberForm= } \\ \mathrm{J}\end{array} & \mathrm{f}, \mathrm{F} 2 & \mathrm{e}, \mathrm{F} 1 \\ 0.5 & 49744.44 & 49740.54 \\ 1.5 & 49752.24 & 49744.44 \\ 2.5 & 49763.93 & 49752.24 \\ 3.5 & 49779.51 & 49763.93 \\ 4.5 & 49798.99 & 49779.51 \\ 5.5 & 49822.37 & 49798.99 \\ 6.5 & 49849.64 & 49822.37 \\ 7.5 & 49880.81 & 49849.64 \\ 8.5 & 49915.86 & 49880.81 \\ 9.5 & 49954.81 & 49915.86\end{array}$

Calculation of line positions for $\mathrm{X}-\mathrm{A}$ transitions

$\ln [60]:=$ EnergyXF2f $\left[\mathbf{v}_{-}, J_{-}\right]:=$Eigenvalues $[$BrownHamiltonf $[\mathrm{v}, \mathrm{J}]][[1]]+$ offset ; EnergyXF1f $\left[v_{-}, J_{-}\right]:=$Eigenvalues [BrownHamiltonf $\left.\left[v_{,}, J\right]\right][[2]]+$ offset ; EnergyXF2e[v_, J_] := Eigenvalues [BrownHamiltone $[\mathrm{v}, \mathrm{J}]][[1]]+$ offset; EnergyXF1e [v_, J_] : = Eigenvalues [BrownHamiltone $[\mathrm{v}, \mathrm{J}]][[2]]+$ offset

Definition of branches 
D. Mathematica notebook for calculating line positions in the $\gamma$-bands of NO

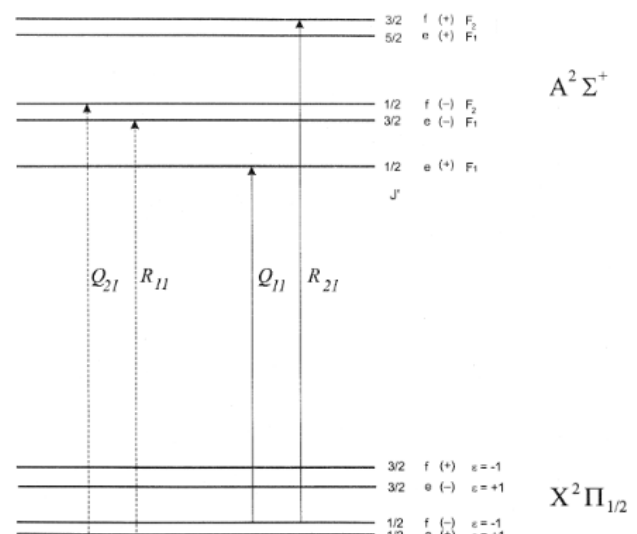

Fig. 1. Energy diagram and symmetry labels of the lowest rotational levels in the $\mathrm{X}^{2} \Pi_{1 / 2}$ and $\mathrm{A}^{2} \Sigma^{+}$states of NO. It should be noted that A-doubling in the electronic ground state and the spin-rotation splitting in the excited state are not dravun to scale. The solid lines indicate

the zero field allowed transitions from the state selected $J=\frac{1}{2} f-$-symmetryy level. The dashed lines indicate transitions that contribute by
mixing of the wavefunctions due to the electric field.

$\ln [64]:=$ P11 [vX_, vA_, J_] : = EnergyAe [VA, J - 1] - EnergyXF1e [vX, J] ; P22 [vX_, $\left.\mathrm{vA}_{-}, J_{-}\right]:=$EnergyAf [vA, J - 1] - EnergyXF2f $[\mathrm{vX}, \mathrm{J}]$; $Q 11\left[v x_{-}, v A \_, J_{-}\right]:=$EnergyAe $[v A, J]-\operatorname{EnergyXF} 1 f[v X, J]$; $\mathrm{Q} 22\left[\mathrm{vX}_{-}, \mathrm{vA}_{-}, J_{-}\right]:=$EnergyAf $[\mathrm{vA}, \mathrm{J}]$ - EnergyXF $2 e[\mathrm{vX}, \mathrm{J}]$;

$R 11\left[v_{-}, v_{-}, J_{-}\right]:=$EnergyAe $[v A, J+1]-$ EnergyXF1e $[v X, J]$; $\mathrm{R} 22\left[\mathrm{vX}, \mathrm{vA}_{-}, \mathrm{J}_{-}\right]:=$EnergyAf $[\mathrm{vA}, \mathrm{J}+1]-$ EnergyXF2f $[\mathrm{vX}, \mathrm{J}]$ P12 [vX_, vA_, J_] : = EnergyAe [vA, J - 1] - EnergyXF2e [vX, J] ; $R 12\left[\mathrm{vX}_{-}, \mathrm{vA}_{-}, J_{-}\right]:=$EnergyAe $[\mathrm{vA}, \mathrm{J}+1]-\operatorname{EnergyXF} 2 \mathrm{e}[\mathrm{vX}, \mathrm{J}]$;

$Q 12\left[\mathrm{vX}_{-}, \mathrm{vA}_{-}, J_{-}\right]:=\operatorname{EnergyAe}[\mathrm{vA}, \mathrm{J}]-\operatorname{EnergyXF} 2 \mathrm{f}[\mathrm{vX}, \mathrm{J}]$; Q21 [vx_, vA_, J_] : = EnergyAf [vA, J] - EnergyXFle $[\mathrm{vX}, J]$ P21 [vX_, vA_, J_] : = EnergyAf [vA, J - 1] - EnergyXF1f $[\mathrm{vX}, J]$; R21 [vX_, vA_, J_] : = EnergyAf [vA, J + 1] - EnergyXF1f [vX, J] ;

$\ln [76]:=\operatorname{Max} J=10.5$

Out[76] $=10 \cdot 5$ 
8 | LinePositionsForThesis3.nb

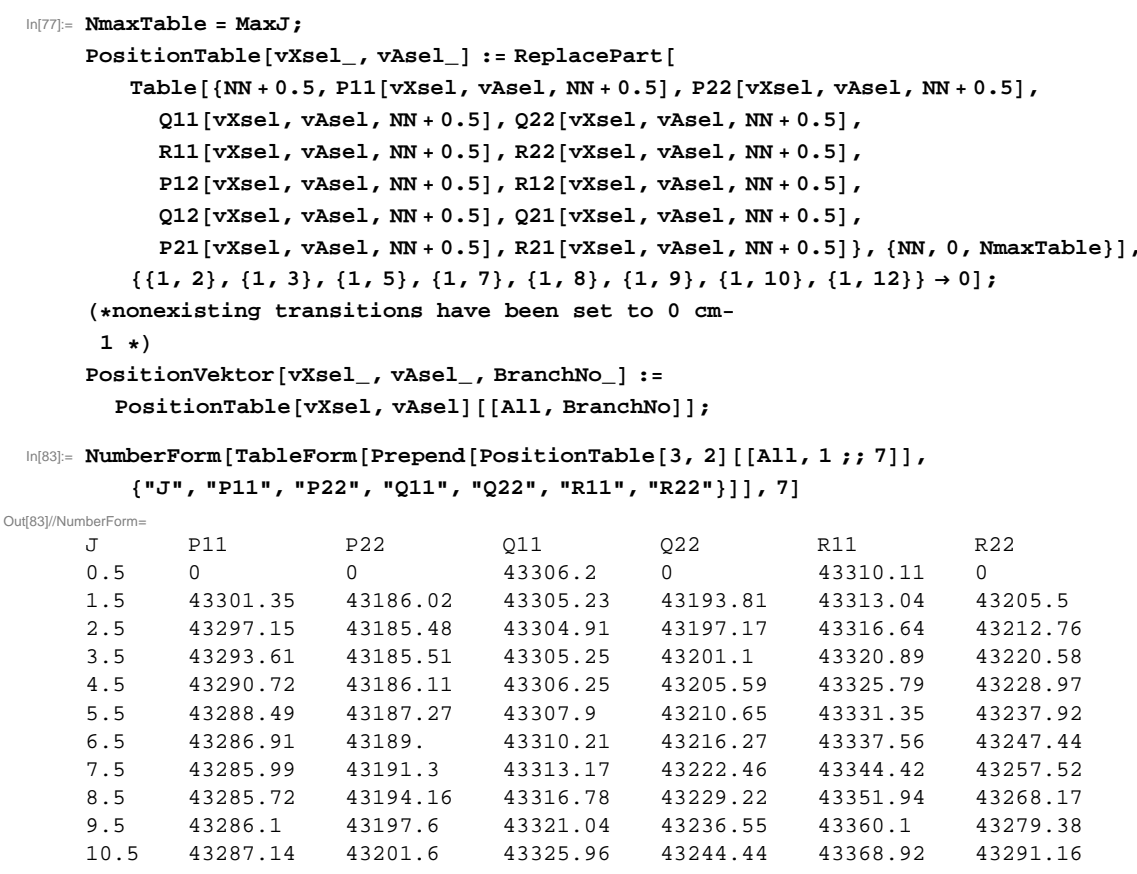

NumberForm [TableForm [Prepend [PositionTable $[3,2][[$ All, $8 ; ;-1]]$,

\{"P12", "R12", "Q12", "Q21", "P21", "R21"\}]]

7] (*Continuation of the table*)

$\begin{array}{llllll}\begin{array}{l}\text { Out[84]/NumberForm }= \\ \text { P12 }\end{array} & \text { R12 } & \text { Q12 } & \text { Q21 } & \text { P21 } & \text { R21 } \\ 0 & 0 & 0 & 43310.11 & 0 & 43317.89 \\ 43182.12 & 43193.81 & 43186.02 & 43313.04 & 43305.23 & 43324.71 \\ 43177.69 & 43197.17 & 43185.48 & 43316.64 & 43304.91 & 43332.19 \\ 43173.82 & 43201.1 & 43185.51 & 43320.89 & 43305.25 & 43340.32 \\ 43170.52 & 43205.59 & 43186.11 & 43325.79 & 43306.25 & 43349.11 \\ 43167.79 & 43210.65 & 43187.27 & 43331.35 & 43307.9 & 43358.55 \\ 43165.62 & 43216.27 & 43189 . & 43337.56 & 43310.21 & 43368.64 \\ 43164.03 & 43222.46 & 43191.3 & 43344.42 & 43313.17 & 43379.39 \\ 43163 . & 43229.22 & 43194.16 & 43351.94 & 43316.78 & 43390.78 \\ 43162.54 & 43236.55 & 43197.6 & 43360.1 & 43321.04 & 43402.83 \\ 43162.66 & 43244.44 & 43201.6 & 43368.92 & 43325.96 & 43415.52\end{array}$


D. Mathematica notebook for calculating line positions in the $\gamma$-bands of NO 


\section{Appendix E}

\section{Further details on the analysis of REMPI spectra from scattering $\mathrm{NO}\left(v_{\mathrm{i}}=11,16\right)$ from $\mathrm{Au}(111)$}

Fitting of the REMPI spectra from scattering $\mathrm{NO}\left(v_{\mathrm{i}}=11,16\right)$ from $\mathrm{Au}(111)$ was performed with predefined rotational state distributions. We used rotational state distributions of the functional form

$$
f_{\mathrm{ROT}}(J)=A_{1} \cdot(2 J+1) \exp \left[-\frac{h c B[v, J] J(J+1)}{k_{B} T_{1}}\right]+B_{1} \exp \left[\frac{-(J-b)^{2}}{2 w^{2}}\right],
$$

where $h$ and $c$ are the Planck constant and the speed of light, respectively. $B[v, J]$ is the rotational constant. The function consists of a Boltzmann part (weighed with $\left.A_{1}\right)$ described by a temperature $\left(T_{1}\right)$ mostly describing the part of the population distribution at low $J$ and a Gaussian function (weighed by $B_{1}$ ) with a width $w$ and a shift in $J$ given by $b$. Parameters were obtained by fitting rotational state distributions (where available) from the analysis of individual rotational lines similar to those shown in Fig. 6.15 and Fig. 6.16. The fitting of the REMPI spectrum was performed with an averaged rotational state distribution at each incidence translational energy. The parameters used for fitting the vibrational state distribution are given in Table E.1 ${ }^{1}$ It should be noted, that the same rotational state distributions are used for fitting

\footnotetext{
${ }^{1}$ The rotational state distributions in Fig. 6.15 and Fig. 6.16 have been converted to a flux. The parameters given in Table E.1 belong to a fit of rotational state distributions as density and are thus a bit hotter.
} 


\section{E. Further details on the analysis of REMPI spectra from scattering $\mathrm{NO}\left(v_{\mathrm{i}}=11,16\right)$ from $\mathrm{Au}(111)$}

the spectra belonging to one incidence orientation and incidence translational energy. Thus, the effect of the vibrational cooling upon vibrational relaxation is negelect. Table E.2 shows which $\gamma_{v^{\prime}, v^{\prime \prime}}$-bands were used for the analysis of the vibrational state distributions and which intensities in each band were obtained from fitting the REMPI spectra. The corresponding table for $v_{\mathrm{i}}=16$ is given in Table E.3.

Average population distributions (in density) are obtained by averaging the the band intensities of $\gamma\left(v^{\prime}, v^{\prime \prime}\right)$ belonging to the same vibrational state $v^{\prime}$ (see Tables E.4 and E.5 for the incidence vibrational states $v_{\mathrm{i}}=11$ and $v_{\mathrm{i}}=16$, respectively).

In order to convert these population distributions into a flux, we use velocity distributions of $\mathrm{NO}$ scattered from $\mathrm{Au}(111)$ in $v_{I}=11$, which have only very recently been measured in our laboratory by Bastian Krüger. This unpublished data shows, that the translation-to-rotation coupling $m_{\mathrm{TR}}$ is similar to the data from Golibrzuch et. al(10) (given by equation 6.2), but that the translation-vibration coupling $m_{\mathrm{TV}}$ is weaker and is for $v_{\mathrm{i}}=11$ given by

$$
m_{\mathrm{TV}}=0.0075-0.111 \cdot E_{\mathrm{trans}}^{\mathrm{i}} / \mathrm{eV}
$$

It is thereby assumed, that NO $v_{\mathrm{i}}=16$ scattering exhibits (for which velocity distributions have not yet been measured) approximately the same translation-to-rotation coupling as molecules scattering in $v_{\mathrm{i}}=11$. As shown in Tables E.4 and E.5, the effect of this correction is very small. The reason so much effort was made to perform this correction was to verify, that it cannot explain the observed population mismatch in the summed scattered vibrational state distributions, when scattering NO molecules $\left(v_{\mathrm{i}}=11\right.$ and 16$)$ with opposite incident orientations from the $\mathrm{Au}(111)$ surface (compare sections 6.2.5 and 6.3.4.

An entire experimental REMPI spectrum when $\mathrm{NO} \mathrm{X}^{2} \Pi_{1 / 2}(v=16, J=0.5)$ scatters from $\mathrm{Au}(111)$ at an incidence translational energy of $0.52 \mathrm{eV}$ (isotropic incidence angular distribution) is shown in Fig. E.1. The figure also shows a simulated spectrum (black line). Spectrum and simulation are binned in intervals of $1 \mathrm{~cm}^{-1}$ (as used for fitting). Although the fit is performed with fixed rotational state distributions, a reasonable agreement between spectrum and simulation is achieved. 
Table E.1: Parameters in the function $f_{\mathrm{ROT}}(J)$ used for fitting vibrational state distributions.

\begin{tabular}{rrrrrrr}
\hline \hline \multicolumn{2}{c}{ Measurement } & $A_{1}$ & $T_{1} / \mathrm{K}$ & $B_{1}$ & $b$ & $w$ \\
\hline$v=11$ & $0.95 \mathrm{eV}$, iso & 0.95 & 553 & 5.34 & 37.3 & 11.0 \\
& $0.69 \mathrm{eV}$, iso & 0.80 & 504 & 7.44 & 37.9 & 11.0 \\
& $0.51 \mathrm{eV}$, iso & 0.52 & 906 & 11.64 & 35.0 & 6.0 \\
$0.14 \mathrm{eV}$, iso & 1.40 & 594 & 0.00 & - & - \\
& $0.05 \mathrm{eV}$, iso & 2.59 & 320 & 0.00 & - & - \\
& $0.51 \mathrm{eV}, \mathrm{O}$-first & 0.37 & 895 & 15.69 & 34.7 & 6.1 \\
& $0.51 \mathrm{eV}, \mathrm{N}$-first & 0.72 & 696 & 10.32 & 34.2 & 6.1 \\
\hline$v=16$ & $0.97 \mathrm{eV}$, iso & 1.75 & 1238 & 6.66 & 50.0 & 8.0 \\
& $0.66 \mathrm{eV}$, iso & 1.58 & 1179 & 7.42 & 46.9 & 8.0 \\
& $0.52 \mathrm{eV}$, iso & 1.35 & 1344 & 8.06 & 40.0 & 8.0 \\
$0.23 \mathrm{eV}$, iso & 1.10 & 1070 & 0.00 & - & - \\
& $0.05 \mathrm{eV}$, iso & 3.25 & 430 & 0.00 & - & - \\
& $0.52 \mathrm{eV}, \mathrm{O}$-first & 1.35 & 1344 & 8.06 & 40.0 & 8.0 \\
$0.52 \mathrm{eV}, \mathrm{N}$-first & 1.35 & 1344 & 8.06 & 40.0 & 8.0 \\
\hline \hline
\end{tabular}


Table E.2: Band intensities from fitting the REMPI spectra when scattering $\mathrm{NO} \mathrm{X}^{2} \Pi_{1 / 2}(v=$ $11, J=0.5)$ from $\mathrm{Au}(111)$. The bands $\gamma(2,4), \gamma(3,5), \gamma(0,6), \gamma(0,7), \gamma(6,9), \gamma(5,9), \gamma(7,10)$, $\gamma(6,10), \gamma(5,10), \gamma(2,10), \gamma(7,11), \gamma(6,11), \gamma(5,11), \gamma(6,12), \gamma(7,12), \gamma(8,12)$ were also fitted, but results were not included in the final evaluation of population distributions.

\begin{tabular}{|c|c|c|c|c|c|c|c|c|c|}
\hline \multicolumn{3}{|c|}{ band assignment } & \multicolumn{7}{|c|}{ fitted band intensities } \\
\hline$v$ & $\gamma$-band & $\tilde{\nu} / \mathrm{cm}^{-1}$ & $\begin{array}{r}0.95 \mathrm{eV} \\
\text { iso }\end{array}$ & $\begin{array}{r}0.69 \mathrm{eV} \\
\text { iso }\end{array}$ & $\begin{array}{r}0.51 \mathrm{eV} \\
\text { iso }\end{array}$ & $\begin{array}{r}0.14 \mathrm{eV} \\
\text { iso }\end{array}$ & $\begin{array}{r}0.05 \mathrm{eV} \\
\text { iso }\end{array}$ & $\begin{array}{r}0.51 \mathrm{eV} \\
\mathrm{O}-\text { first }\end{array}$ & $\begin{array}{r}0.51 \mathrm{eV} \\
\mathrm{N}-\text { first }\end{array}$ \\
\hline 1 & $\gamma(0,1)$ & 42322.9 & 3.73 & 8.60 & 1.36 & 2.99 & 2.13 & 1.54 & 1.26 \\
\hline 2 & $\gamma(0,2)$ & 40474.9 & 1.52 & 2.09 & 2.79 & 2.20 & 2.83 & 2.55 & 2.67 \\
\hline \multirow[t]{2}{*}{3} & $\gamma(0,3)$ & 38655.0 & 4.77 & 4.05 & 4.94 & 4.98 & 5.41 & 4.33 & 5.02 \\
\hline & $\gamma(1,3)$ & 40996.9 & 1.89 & 4.66 & 2.51 & 3.67 & 3.89 & 2.22 & 1.84 \\
\hline \multirow[t]{2}{*}{4} & $\gamma(0,4)$ & 36863.0 & 5.62 & 3.94 & 4.02 & 3.66 & 3.34 & 3.70 & 4.39 \\
\hline & $\gamma(1,4)$ & 39205.0 & 6.36 & 4.92 & 4.42 & 4.24 & 3.54 & 4.18 & 5.23 \\
\hline \multirow[t]{3}{*}{5} & $\gamma(0,5)$ & 35099.0 & 6.14 & 2.93 & 4.64 & 3.51 & 4.01 & 3.85 & 4.91 \\
\hline & $\gamma(1,5)$ & 37441.0 & 8.75 & 7.58 & 6.93 & 4.98 & 3.36 & 5.76 & 6.81 \\
\hline & $\gamma(2,5)$ & 39750.2 & 4.31 & 2.61 & 4.58 & 3.56 & 1.30 & 5.02 & 5.27 \\
\hline \multirow[t]{2}{*}{6} & $\gamma(1,6)$ & 35704.9 & 7.19 & 3.84 & 5.41 & 5.82 & 5.02 & 5.02 & 5.83 \\
\hline & $\gamma(2,6)$ & 38014.2 & 6.25 & 6.62 & 5.80 & 6.92 & 4.46 & 5.30 & 3.95 \\
\hline \multirow[t]{4}{*}{7} & $\gamma(1,7)$ & 33996.7 & 6.64 & 2.99 & 5.69 & 4.30 & 4.96 & 5.53 & 5.12 \\
\hline & $\gamma(2,7)$ & 36306.0 & 5.41 & 4.90 & 6.31 & 3.43 & 1.00 & 6.63 & 5.85 \\
\hline & $\gamma(3,7)$ & 38582.4 & 3.93 & 3.13 & 4.85 & 2.55 & 2.65 & 5.06 & 4.16 \\
\hline & $\gamma(4,7)$ & 40825.7 & 2.75 & 4.29 & 3.39 & 3.33 & 2.73 & 3.79 & 2.93 \\
\hline \multirow[t]{4}{*}{8} & $\gamma(1,8)$ & 32316.5 & 2.46 & 3.96 & 2.80 & 2.55 & 4.05 & 3.68 & 2.00 \\
\hline & $\gamma(2,8)$ & 34625.8 & 4.24 & 2.35 & 3.25 & 4.24 & 5.30 & 3.76 & 2.57 \\
\hline & $\gamma(3,8)$ & 36902.2 & 3.28 & 3.47 & 4.87 & 3.89 & 4.19 & 5.92 & 3.98 \\
\hline & $\gamma(5,8)$ & 41355.5 & 1.44 & 7.18 & 3.32 & 2.34 & 3.82 & 3.70 & 2.42 \\
\hline \multirow[t]{3}{*}{9} & $\gamma(2,9)$ & 32973.5 & 2.22 & 1.59 & 2.59 & 3.27 & 3.70 & 3.74 & 1.64 \\
\hline & $\gamma(3,9)$ & 35249.9 & 2.50 & 2.38 & 2.57 & 4.40 & 5.42 & 3.49 & 1.82 \\
\hline & $\gamma(4,9)$ & 37493.2 & 2.22 & 2.59 & 2.91 & 2.99 & 2.07 & 3.28 & 2.19 \\
\hline \multirow[t]{2}{*}{10} & $\gamma(3,10)$ & 33625.6 & 1.71 & 1.62 & 2.09 & 3.10 & 4.50 & 3.21 & 1.07 \\
\hline & $\gamma(4,10)$ & 35868.9 & 1.47 & 2.18 & 1.79 & 3.59 & 4.02 & 2.58 & 1.63 \\
\hline \multirow[t]{2}{*}{11} & $\gamma(3,11)$ & 32029.4 & 0.61 & 2.20 & 2.46 & 3.11 & 5.75 & 3.71 & 1.17 \\
\hline & $\gamma(4,11)$ & 34272.7 & 1.89 & 2.07 & 2.35 & 5.07 & 6.17 & 3.24 & 1.48 \\
\hline \multirow[t]{2}{*}{12} & $\gamma(4,12)$ & 32704.6 & 0.27 & 0.00 & 0.60 & 0.21 & 0.20 & 0.86 & 0.28 \\
\hline & $\gamma(5,12)$ & 34914.6 & 0.45 & 1.27 & 0.76 & 1.07 & 0.18 & 0.88 & 0.66 \\
\hline
\end{tabular}


Table E.3: Band intensities from fitting the REMPI spectra when scattering $\mathrm{NO} \mathrm{X}^{2} \Pi_{1 / 2}(v=$ $16, J=0.5)$ from $\mathrm{Au}(111)$. Again, several more $\gamma\left(v^{\prime \prime}, v^{\prime}\right)$-bands were also used for fitting, but not for averaging to obtain vibrational state distributions. The table is continuued on the next page.

\begin{tabular}{|c|c|c|c|c|c|c|c|c|c|}
\hline \multicolumn{3}{|c|}{ band assignment } & \multicolumn{7}{|c|}{ fitted band intensities } \\
\hline & & & $0.97 \mathrm{eV}$ & $0.66 \mathrm{eV}$ & $0.52 \mathrm{eV}$ & $0.23 \mathrm{eV}$ & $0.05 \mathrm{eV}$ & $0.52 \mathrm{eV}$ & $0.52 \mathrm{eV}$ \\
\hline$v$ & $\gamma$-band & $\tilde{\nu} / \mathrm{cm}^{-1}$ & iso & iso & iso & iso & iso & O-first & $\mathrm{N}$-first \\
\hline 2 & $\gamma(0,2)$ & 40474.9 & 1.42 & 3.50 & 1.53 & 3.36 & 3.19 & 1.81 & 1.78 \\
\hline 3 & $\gamma(0,3)$ & 38655.0 & 3.75 & 3.36 & 2.86 & 2.33 & 4.13 & 3.09 & 2.96 \\
\hline \multirow[t]{2}{*}{4} & $\gamma(0,4)$ & 36863.0 & 5.45 & 3.01 & 5.76 & 5.34 & 2.06 & 6.31 & 5.39 \\
\hline & $\gamma(1,4)$ & 39205.0 & 4.10 & 5.26 & 4.09 & 4.50 & 5.32 & 2.30 & 2.24 \\
\hline \multirow[t]{3}{*}{5} & $\gamma(0,5)$ & 35099.0 & 5.21 & 6.36 & 7.27 & 1.09 & 5.08 & 7.49 & 4.51 \\
\hline & $\gamma(1,5)$ & 37441.0 & 4.77 & 3.88 & 6.27 & 7.41 & 2.48 & 7.01 & 8.12 \\
\hline & $\gamma(2,5)$ & 39750.2 & 3.33 & 2.90 & 2.89 & 3.54 & 2.49 & 2.86 & 1.93 \\
\hline \multirow[t]{2}{*}{6} & $\gamma(1,6)$ & 35704.9 & 7.93 & 9.99 & 8.17 & 7.36 & 6.89 & 9.35 & 8.56 \\
\hline & $\gamma(2,6)$ & 38014.2 & 5.47 & 3.17 & 5.77 & 7.19 & 4.24 & 6.33 & 6.02 \\
\hline \multirow[t]{3}{*}{7} & $\gamma(1,7)$ & 33996.7 & 4.79 & 4.04 & 4.17 & 6.15 & 4.72 & 7.34 & 6.99 \\
\hline & $\gamma(2,7)$ & 36306.0 & 6.58 & 2.99 & 7.63 & 6.41 & 3.98 & 8.85 & 6.10 \\
\hline & $\gamma(3,7)$ & 38582.4 & 3.99 & 3.43 & 2.94 & 1.23 & 2.38 & 3.47 & 3.58 \\
\hline \multirow[t]{3}{*}{8} & $\gamma(1,8)$ & 32316.5 & 2.70 & 6.71 & 4.61 & 3.85 & 3.45 & 5.60 & 5.15 \\
\hline & $\gamma(2,8)$ & 34625.8 & 3.81 & 3.80 & 3.42 & 3.76 & 5.09 & 5.97 & 4.51 \\
\hline & $\gamma(3,8)$ & 36902.2 & 5.25 & 2.33 & 5.05 & 4.15 & 1.26 & 6.93 & 5.24 \\
\hline \multirow[t]{5}{*}{9} & $\gamma(1,9)$ & 30664.2 & 2.18 & 2.05 & 1.14 & 2.13 & 1.73 & 1.43 & 0.79 \\
\hline & $\gamma(2,9)$ & 32973.5 & 2.82 & 6.92 & 3.24 & 3.48 & 3.81 & 3.16 & 3.07 \\
\hline & $\gamma(3,9)$ & 35249.9 & 3.93 & 6.29 & 4.51 & 2.01 & 6.06 & 5.45 & 2.36 \\
\hline & $\gamma(4,9)$ & 37493.2 & 2.55 & 2.05 & 2.32 & 4.12 & 3.11 & 3.42 & 3.27 \\
\hline & $\gamma(5,9)$ & 39703.2 & 1.18 & 0.23 & 0.58 & 0.55 & 1.43 & 0.63 & 0.28 \\
\hline \multirow[t]{2}{*}{10} & $\gamma(2,10)$ & 31349.2 & 0.54 & 2.76 & 0.95 & 2.00 & 1.23 & 0.98 & 0.73 \\
\hline & $\gamma(4,10)$ & 35868.9 & 3.26 & 2.35 & 2.64 & 3.16 & 4.28 & 3.41 & 2.09 \\
\hline \multirow[t]{3}{*}{11} & $\gamma(3,11)$ & 32029.4 & 0.68 & 1.32 & 1.16 & 1.39 & 1.84 & 1.56 & 0.88 \\
\hline & $\gamma(4,11)$ & 34272.7 & 1.97 & 1.32 & 0.29 & 1.46 & 4.01 & 1.14 & 0.02 \\
\hline & $\gamma(5,11)$ & 36482.7 & 2.06 & 0.36 & 0.47 & 0.37 & 0.64 & 1.06 & 0.75 \\
\hline
\end{tabular}


E. Further details on the analysis of REMPI spectra from scattering $\mathrm{NO}\left(v_{\mathrm{i}}=11,16\right)$ from $\mathrm{Au}(111)$

Table continued from last page.

\begin{tabular}{|c|c|c|c|c|c|c|c|c|c|}
\hline \multicolumn{3}{|c|}{ band assignment } & \multicolumn{7}{|c|}{ fitted band intensities } \\
\hline$v$ & $\gamma$-band & $\tilde{\nu} / \mathrm{cm}^{-1}$ & $\begin{array}{r}0.97 \mathrm{eV} \\
\text { iso }\end{array}$ & $\begin{array}{r}0.66 \mathrm{eV} \\
\text { iso }\end{array}$ & $\begin{array}{r}0.52 \mathrm{eV} \\
\text { iso }\end{array}$ & $\begin{array}{r}0.23 \mathrm{eV} \\
\text { iso }\end{array}$ & $\begin{array}{r}0.05 \mathrm{eV} \\
\text { iso }\end{array}$ & $\begin{array}{l}0.52 \mathrm{eV} \\
\mathrm{O}-\text { first }\end{array}$ & $\begin{array}{c}0.52 \mathrm{eV} \\
\mathrm{N}-\text { first }\end{array}$ \\
\hline \multirow[t]{3}{*}{12} & $\gamma(3,12)$ & 30461.3 & 0.64 & 1.11 & 0.20 & 1.06 & 1.12 & 0.50 & 0.53 \\
\hline & $\gamma(4,12)$ & 32704.6 & 1.03 & 1.92 & 1.48 & 1.01 & 1.49 & 1.02 & 1.39 \\
\hline & $\gamma(5,12)$ & 34914.6 & 1.51 & 0.98 & 1.57 & 0.90 & 2.25 & 1.17 & 0.60 \\
\hline \multirow[t]{3}{*}{13} & $\gamma(4,13)$ & 31164.9 & 0.42 & 1.11 & 0.43 & 0.81 & 1.03 & 0.70 & 0.42 \\
\hline & $\gamma(5,13)$ & 33374.8 & 0.02 & 0.00 & 1.82 & 0.40 & 0.64 & 2.15 & 1.81 \\
\hline & $\gamma(6,13)$ & 35551.3 & 1.22 & 0.99 & 0.00 & 0.32 & 0.07 & 0.53 & 0.86 \\
\hline \multirow[t]{3}{*}{14} & $\gamma(4,14)$ & 29653.5 & 0.06 & 0.13 & 0.08 & 0.12 & 0.11 & 0.10 & 0.18 \\
\hline & $\gamma(5,14)$ & 31863.5 & 0.00 & 0.15 & 0.28 & 0.11 & 0.78 & 0.42 & 0.10 \\
\hline & $\gamma(6,14)$ & 34039.9 & 1.41 & 0.00 & 0.07 & 1.67 & 1.73 & 1.22 & 0.43 \\
\hline \multirow[t]{3}{*}{15} & $\gamma(5,15)$ & 30380.9 & 0.67 & 0.17 & 0.14 & 0.40 & 0.50 & 0.29 & 0.39 \\
\hline & $\gamma(6,15)$ & 32557.3 & 0.26 & 0.75 & 1.11 & 0.32 & 0.80 & 0.73 & 0.78 \\
\hline & $\gamma(7,15)$ & 34699.9 & 0.83 & 0.77 & 1.04 & 1.90 & 1.56 & 2.41 & 1.35 \\
\hline \multirow[t]{2}{*}{16} & $\gamma(6,16)$ & 31103.5 & 1.08 & 0.94 & 0.38 & 1.44 & 1.56 & 0.55 & 0.41 \\
\hline & $\gamma(7,16)$ & 33246.2 & 0.69 & 0.51 & 1.39 & 1.05 & 0.84 & 2.02 & 1.39 \\
\hline \multirow[t]{2}{*}{17} & $\gamma(6,17)$ & 29679.0 & 0.13 & 0.00 & 0.00 & 0.06 & 0.14 & 0.01 & 0.02 \\
\hline & $\gamma(7,17)$ & 31821.7 & 0.31 & 0.09 & 0.28 & 0.10 & 0.46 & 0.41 & 0.15 \\
\hline
\end{tabular}


Table E.4: Vibrational state distributions generated when $\mathrm{NO} \mathrm{X}^{2} \Pi_{1 / 2}(v=11, J=$ $0.5)$ scatters from $\mathrm{Au}(111)$ at different incidence translational energies and orientations. The distributions are given as density (which is the average of the band intensities from Table E.2 and as a flux (after applying th density-to-flux conversion). Distributions are normalized to the summed population in the vibrational state $v=2-11$, because population in $v=1$ has a high error and population in $v=0$ can not be detected.

\begin{tabular}{rrrrrrrr}
\hline \hline & $\begin{array}{r}0.97 \mathrm{eV} \\
v\end{array}$ & $\begin{array}{r}0.69 \mathrm{eV} \\
\text { iso }\end{array}$ & $\begin{array}{r}0.51 \mathrm{eV} \\
\text { iso }\end{array}$ & $\begin{array}{c}0.14 \mathrm{eV} \\
\text { iso }\end{array}$ & $\begin{array}{r}0.05 \mathrm{eV} \\
\text { iso }\end{array}$ & $\begin{array}{r}0.51 \mathrm{eV} \\
\text { iso-first }\end{array}$ & $\begin{array}{c}0.51 \mathrm{eV} \\
\text { N-first }\end{array}$ \\
\cline { 2 - 8 } 1 & 0.102 & 0.247 & 0.036 & 0.078 & 0.054 & 0.041 & 0.034 \\
2 & 0.041 & 0.060 & 0.075 & 0.057 & 0.071 & 0.068 & 0.071 \\
3 & 0.091 & 0.125 & 0.100 & 0.112 & 0.117 & 0.088 & 0.092 \\
4 & 0.163 & 0.127 & 0.113 & 0.103 & 0.087 & 0.105 & 0.129 \\
5 & 0.175 & 0.126 & 0.144 & 0.104 & 0.073 & 0.131 & 0.152 \\
6 & 0.183 & 0.150 & 0.150 & 0.165 & 0.120 & 0.138 & 0.131 \\
7 & 0.128 & 0.110 & 0.135 & 0.088 & 0.071 & 0.141 & 0.121 \\
8 & 0.078 & 0.122 & 0.095 & 0.084 & 0.109 & 0.114 & 0.073 \\
9 & 0.063 & 0.063 & 0.072 & 0.092 & 0.094 & 0.094 & 0.050 \\
10 & 0.043 & 0.055 & 0.052 & 0.087 & 0.107 & 0.078 & 0.036 \\
11 & 0.034 & 0.061 & 0.064 & 0.106 & 0.150 & 0.093 & 0.035 \\
\hline & & & Population as & flux & & \\
1 & 0.114 & 0.278 & 0.041 & 0.088 & 0.062 & 0.046 & 0.038 \\
2 & 0.045 & 0.066 & 0.082 & 0.064 & 0.080 & 0.075 & 0.079 \\
3 & 0.097 & 0.135 & 0.107 & 0.122 & 0.129 & 0.094 & 0.099 \\
4 & 0.171 & 0.134 & 0.119 & 0.109 & 0.093 & 0.111 & 0.135 \\
5 & 0.178 & 0.129 & 0.148 & 0.108 & 0.076 & 0.134 & 0.156 \\
6 & 0.183 & 0.151 & 0.150 & 0.168 & 0.122 & 0.139 & 0.131 \\
7 & 0.124 & 0.108 & 0.132 & 0.087 & 0.071 & 0.138 & 0.118 \\
8 & 0.074 & 0.116 & 0.091 & 0.081 & 0.106 & 0.109 & 0.070 \\
9 & 0.058 & 0.058 & 0.067 & 0.087 & 0.089 & 0.087 & 0.047 \\
10 & 0.039 & 0.049 & 0.047 & 0.079 & 0.099 & 0.070 & 0.033 \\
11 & 0.030 & 0.054 & 0.056 & 0.094 & 0.134 & 0.082 & 0.031 \\
\hline \hline
\end{tabular}


Table E.5: Same as Table E.4 but for $\mathrm{NO} \mathrm{X}^{2} \Pi_{1 / 2}(v=16, J=0.5)$.

\begin{tabular}{|c|c|c|c|c|c|c|c|}
\hline$v$ & $\begin{array}{r}0.97 \mathrm{eV} \\
\text { iso }\end{array}$ & $\begin{array}{r}0.69 \mathrm{eV} \\
\text { iso } \\
\end{array}$ & $\begin{array}{r}0.51 \mathrm{eV} \\
\text { iso } \\
\end{array}$ & $\begin{array}{r}0.14 \mathrm{eV} \\
\text { iso }\end{array}$ & $\begin{array}{r}0.05 \mathrm{eV} \\
\text { iso } \\
\end{array}$ & $\begin{array}{c}0.51 \mathrm{eV} \\
\mathrm{O}-\text {-first }\end{array}$ & $\begin{array}{r}0.51 \mathrm{eV} \\
\mathrm{N}-\text { first }\end{array}$ \\
\hline & \multicolumn{7}{|c|}{ Population as density } \\
\hline 2 & 0.037 & 0.095 & 0.040 & 0.090 & 0.086 & 0.048 & 0.047 \\
\hline 3 & 0.098 & 0.092 & 0.076 & 0.062 & 0.111 & 0.081 & 0.078 \\
\hline 4 & 0.125 & 0.113 & 0.130 & 0.132 & 0.100 & 0.113 & 0.101 \\
\hline 5 & 0.116 & 0.119 & 0.144 & 0.107 & 0.090 & 0.153 & 0.128 \\
\hline 6 & 0.175 & 0.179 & 0.184 & 0.194 & 0.150 & 0.207 & 0.192 \\
\hline 7 & 0.134 & 0.095 & 0.130 & 0.123 & 0.100 & 0.173 & 0.146 \\
\hline 8 & 0.102 & 0.117 & 0.115 & 0.105 & 0.088 & 0.163 & 0.131 \\
\hline 9 & 0.066 & 0.096 & 0.062 & 0.066 & 0.087 & 0.074 & 0.052 \\
\hline 10 & 0.050 & 0.070 & 0.047 & 0.069 & 0.074 & 0.058 & 0.037 \\
\hline 11 & 0.041 & 0.027 & 0.017 & 0.029 & 0.058 & 0.033 & 0.014 \\
\hline 12 & 0.028 & 0.036 & 0.028 & 0.026 & 0.044 & 0.024 & 0.022 \\
\hline 13 & 0.014 & 0.019 & 0.020 & 0.014 & 0.016 & 0.030 & 0.027 \\
\hline 14 & 0.013 & 0.003 & 0.004 & 0.017 & 0.024 & 0.015 & 0.006 \\
\hline 15 & 0.015 & 0.015 & 0.020 & 0.023 & 0.026 & 0.030 & 0.022 \\
\hline \multirow[t]{2}{*}{16} & 0.023 & 0.020 & 0.023 & 0.033 & 0.032 & 0.034 & 0.024 \\
\hline & \multicolumn{7}{|c|}{ Population as flux } \\
\hline 2 & 0.041 & 0.106 & 0.045 & 0.100 & 0.096 & 0.053 & 0.052 \\
\hline 3 & 0.106 & 0.100 & 0.082 & 0.068 & 0.122 & 0.088 & 0.085 \\
\hline 4 & 0.133 & 0.120 & 0.138 & 0.141 & 0.107 & 0.121 & 0.107 \\
\hline 5 & 0.121 & 0.125 & 0.150 & 0.113 & 0.096 & 0.159 & 0.133 \\
\hline 6 & 0.179 & 0.184 & 0.188 & 0.200 & 0.155 & 0.211 & 0.196 \\
\hline 7 & 0.134 & 0.095 & 0.129 & 0.124 & 0.101 & 0.173 & 0.146 \\
\hline 8 & 0.100 & 0.114 & 0.112 & 0.103 & 0.087 & 0.159 & 0.128 \\
\hline 9 & 0.063 & 0.092 & 0.059 & 0.063 & 0.084 & 0.071 & 0.049 \\
\hline 10 & 0.046 & 0.065 & 0.044 & 0.065 & 0.071 & 0.054 & 0.035 \\
\hline 11 & 0.038 & 0.025 & 0.015 & 0.026 & 0.054 & 0.030 & 0.013 \\
\hline 12 & 0.025 & 0.033 & 0.025 & 0.024 & 0.040 & 0.021 & 0.020 \\
\hline 13 & 0.013 & 0.017 & 0.017 & 0.012 & 0.014 & 0.026 & 0.024 \\
\hline 14 & 0.011 & 0.002 & 0.003 & 0.014 & 0.020 & 0.013 & 0.005 \\
\hline 15 & 0.013 & 0.013 & 0.017 & 0.019 & 0.022 & 0.025 & 0.018 \\
\hline 16 & 0.019 & 0.016 & 0.019 & 0.027 & 0.026 & 0.027 & 0.019 \\
\hline
\end{tabular}



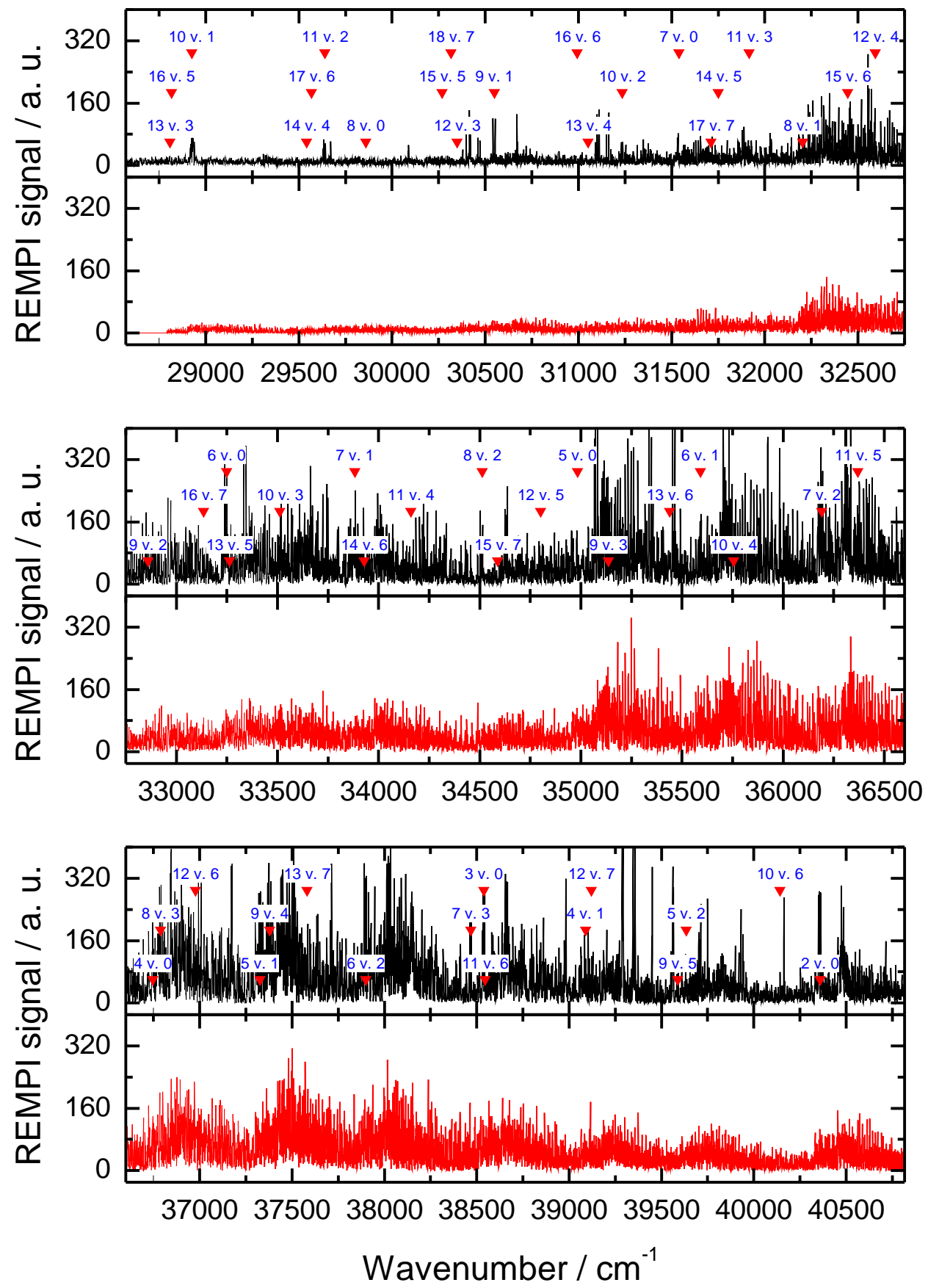

Figure E.1: Comparison of a measured spectrum to a fit. - The figure shows a REMPI spectrum (black color) of $\mathrm{NO} \mathrm{X}^{2} \Pi_{1 / 2}(v=16, J=0.5$ scattered from $\mathrm{Au}(111)$ at an incidence translational energy of $0.52 \mathrm{eV}$ with an isotropic rotational state distributions. The simulation (red color) has been performed assuming identical rotational state distributions in all vibrational states. Nevertheless, a reasonable agreement between spectrum and simulation is obtained. This allows for the determination of the vibrational state distributions. Figure adapted from the Appendix of Ref. (6). High intensity peaks in the experimental spectrum are due to molecules from the incoming molecular beam; an effect that cannot be completely suppressed by the experiment. 


\section{List of publications}

1. K. Golibrzuch, N. Bartels, D. J. Auerbach, A. M. Wodtke, The dynamics of molecular interactions and chemical reactions at metal surfaces: testing the foundations of theory, Annu. Rev. Phys. Chem., doi: 10.1146/annurevphyschem-040214-121958, accepted.

2. B. Krüger, N. Bartels, C. Bartels, A. Kandratsenka, J. Tully, A. Wodtke, T. Schäfer, NO vibrational energy transfer on a metal surface: Still a challenge to first principles theory, J. Phys. Chem. C, 119:3268 (2015).

3. N. Bartels, B. C. Krüger, D. J. Auerbach, A. M. Wodtke, T. Schäfer, Controlling an electron-transfer reaction at a metal surface by manipulating reactant motion and orientation, Angew. Chem. Int. Ed., 53(50):13690 (2014); Steuerung einer Elektronentransferreaktion an einer Metalloberfläche durch Manipulation der Bewegung und Orientierung der Reaktanten, Angew. Chem., 126(50):13908 (2014).

4. N. Bartels, K. Golibrzuch, C. Bartels, L. Chen, D. J. Auerbach, A. M. Wodtke, T. Schäfer, Dynamical steering in an electron transfer surface reaction: Oriented $\mathrm{NO}\left(\mathrm{v}=3,0.08<\mathrm{E}_{\mathrm{I}}<0.89 \mathrm{eV}\right)$ relaxation in collisions with a Au(111) surface, J. Chem. Phys., 140(5):054710 (2014).

5. N. Bartels, B. C. Krüger, S. Meyer, A. M. Wodtke, T. Schäfer, Suppression of spontaneous emission in the optical pumping of molecules: Pumpdump-sweep-probe, J. Phys. Chem. Lett., 4(14):2367 (2013).

6. N. Bartels, K. Golibrzuch, C. Bartels, L. Chen, D. J. Auerbach, A. M. Wodtke, T. Schäfer, Observation of orientation-dependent electron transfer in molecule-surface collisions, PNAS, 110(44):17738 (2013). 
7. T. Schäfer, N. Bartels, K. Golibrzuch, C. Bartels, H. Köckert, D. J. Auerbach, T. N. Kitsopoulos, A. M. Wodtke, Observation of direct vibrational excitation in gas-surface collisions of $\mathrm{CO}$ with $\mathrm{Au}(111)$ : a new model system for surface dynamics, Phys. Chem. Chem. Phys., 15(6):1863 (2013).

8. T. Schäfer, N. Bartels, N. Hocke, X. Yang, A. M. Wodtke, Orienting polar molecules without hexapoles: Optical state selection with adiabatic orientation, Chem. Phys. Lett., 535:1 (2012).

9. N. Bartels, T. Schäfer, J. Hühnert, R. W. Field, A. M. Wodtke, Production of a beam of highly vibrationally excited CO using perturbations, $J$. Chem. Phys., 136(21):214201 (2012).

10. P. W. Lohse, N. Bartels, A. Stoppa, R. Buchner, T. Lenzer, K. Oum, Dielectric relaxation and ultrafast transient absorption spectroscopy of $\left[\mathbf{C}_{\mathbf{6}} \mathbf{m i m}\right]^{+}\left[\mathbf{T f}_{\mathbf{2}} \mathbf{N}\right]^{-}$/acetonitrile mixtures, Phys. Chem. Chem. Phys., 14(10): 3596 (2012).

11. C. Hellwig, N. Bartels, K. Salffner, B. Siepchen, C. Weber, H. Krassen, Ist unser Klima noch zu retten?,Bunsenmagazin, 11(1):14 (2009). 